\title{
Experimental Study of Three-body Cabibbo-suppressed D0 Decays and Extraction of CP Violation Parameters
}

\author{
Kalanand Mishra
}

SLAC-R-893

Prepared for the Department of Energy

under contract number DE-AC02-76SF00515

Printed in the United States of America. Available from the National Technical Information Service, U.S. Department of Commerce, 5285 Port Royal Road, Springfield, VA 22161. 
This document, and the material and data contained therein, was developed under sponsorship of the United States Government. Neither the United States nor the Department of Energy, nor the Leland Stanford Junior University, nor their employees, nor their respective contractors, subcontractors, or their employees, makes an warranty, express or implied, or assumes any liability of responsibility for accuracy, completeness or usefulness of any information, apparatus, product or process disclosed, or represents that its use will not infringe privately owned rights. Mention of any product, its manufacturer, or suppliers shall not, nor is it intended to, imply approval, disapproval, or fitness of any particular use. A royalty-free, nonexclusive right to use and disseminate same of whatsoever, is expressly reserved to the United States and the University. 


\title{
Experimental Study of Three-body Cabibbo-suppressed $D^{0}$ Decays and Extraction of $\boldsymbol{C P}$ Violation Parameters
}

\author{
Kalanand Mishra \\ M.S., Physics \\ Jawaharlal Nehru University, New Delhi, India
}

A dissertation submitted to the

Graduate School

of the University of Cincinnati

in partial fulfillment of the

requirements for the degree of

DOCTORATE OF PHILOSOPHY (Ph.D.)

in the department of Physics

Committee Chairs: Professors Brian T. Meadows and Michael D. Sokoloff 


\section{UNIVERSITY OF CINCINNATI}

Date: January 22, 2008

I, Kalanand Mishra, hereby submit this as part of the requirements for the degree of:

Doctor of Philosophy

in:

$\underline{\text { Physics. }}$

It is entitled:

Experimental Study of Three-body Cabibbo-suppressed $D^{0}$

Decays and Extraction of $C P$ Violation Parameters.

This work and its defense approved by:

$\underline{\text { Prof. Brian T. Meadows, Co-chair }}$

$\underline{\text { Prof. Michael D. Sokoloff, Co-chair }}$

$\underline{\text { Prof. Alexander L. Kagan }}$

$\underline{\text { Prof. Andrei B. Kogan }}$

Prof. Alan J. Schwartz 


\begin{abstract}
I present measurements of the relative branching ratios, Dalitz plot structures and $C P$-asymmetry values in the three-body singly Cabibbo-suppressed decays $D^{0} \rightarrow \pi^{-} \pi^{+} \pi^{0}$ and $D^{0} \rightarrow K^{-} K^{+} \pi^{0}$ using data collected by the BABAR detector at the PEP-II asymmetric-energy ring at SLAC. I apply the results of the $D^{0} \rightarrow \pi^{-} \pi^{+} \pi^{0}$ analysis to extracting $C P$-violation parameters related to the CKM angle $\gamma$ (or $\phi_{3}$ ) using the decay $B^{-} \rightarrow D_{\pi^{+} \pi^{-} \pi^{0}} K^{-}$.
\end{abstract}




\section{Acknowledgments}

I express my sincere gratitude to the Department of Physics at the University of Cincinnati for giving me the opportunity to pursue graduate study in physics. My special thanks go to my thesis advisers Professors Brian Meadows and Mike Sokoloff for guiding me through my research and helping me generously, both professionally and personally. I thank the BABAR collaborators for welcoming me to the collaboration and to the SLAC technical staff members for the courtesy and support they have given me. My research has been financially supported by the National Science Foundation and the running of the BABAR experiment is supported by the US Department of Energy. I would like to thank the US government and American public for welcoming me to the United States.

I would like to express my gratitude to Professors Alex Kagan and Alan Schwartz for teaching me particle physics and field theory and for serving in my thesis committee, and to Prof. Rohana Wijewardana for his lessons in quantum mechanics. I thank Prof. Andrei Kogan for serving in my thesis committee, and to Prof. Kay Kinoshita for agreeing to serve as substitute in case of an absence. I thank Giampiero Mancinelli for his help at various stages of physics analysis, and Rolf Andreassen for always checking for adherence to standard grammar and language uses in my documentations. I am thankful to the dedicated staff members Donna Deutenberg, Melody Whitlock, and Elle Mengon in Cincinnati and Kathy Webb, Donna Hernandez, and Kazuko Onaga in SLAC for their help.

It has been both a privilege and a wonderful experience to work with the following BABAR colleagues: Abi Soffer (Tel Aviv University), Frank Winklmeier (CERN), Jinlong Zhang (Argonne Lab), and Mario Gaspero (University of Rome at La Sapienza). My sincere thanks are due to Bill Dunwoodie, Jonathon Coleman, and Dave Aston (all SLAC); Alexandre Telnov (Princeton); Kevin Flood (University of Wisconsin at Madison); and Michael Wilson (CERN) for closely following my work and making valuable comments and suggestions. I am grateful to Don Summers (University of Mississippi) for reading this document carefully and for making valuable suggestions for its improvement.

I am grateful to my parents and my two sisters for their support and encouragement. I extend my sincere gratitude to my aunt Prabha Jha and uncle Prof. S. Jha and the entire Jha family who have helped me so much and have been there whenever I needed them. Finally, I would like to thank all those individuals who have helped me in various ways over the years. 


\section{Contents}

1 Introduction $\quad 13$

1.1 Standard Model . . . . . . . . . . . . . . . . . . . . . . 13

1.2 Weak interaction . . . . . . . . . . . . . . . . 13

1.3 Charged weak decays . . . . . . . . . . . . . . . . . . . . . . . . . . . . . . . .

1.4 Weak decays of charmed mesons . . . . . . . . . . . . . . 14

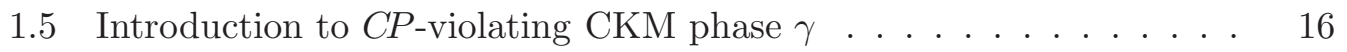

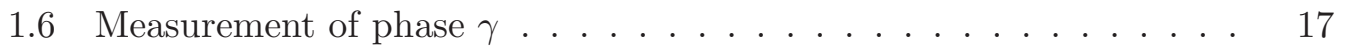

1.7 Search for $C P$ violation in charm decays . . . . . . . . . . . . . . . . 19

$1.8 C P$ asymmetry in Cabibbo-suppressed $D^{0}$ decays . . . . . . . . . . . 19

2 BABAR Detector and Event Reconstruction 20

2.1 The BABAR experiment . . . . . . . . . . . . . . . . . 20

2.2 Detector overview . . . . . . . . . . . . . . . . . . . . . . 22

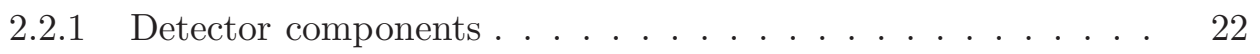

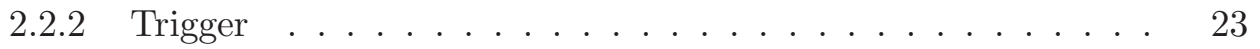

2.2.3 Beam parameters . . . . . . . . . . . . . . 23

2.2.4 Beam background sources . . . . . . . . . . . . . . . . 23

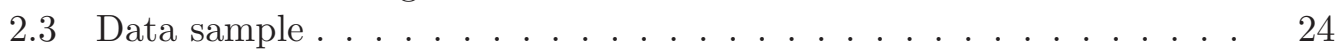

2.4 Charged particle track reconstruction . . . . . . . . . . . . . . 24

$2.5 \pi^{0}$ reconstruction . . . . . . . . . . . . . . . . . . . 25

2.6 Charged particle identification . . . . . . . . . . 25

2.7 Event reconstruction . . . . . . . . . . . . . . . . . . . 25

2.8 Dealing with multiple candidates . . . . . . . . . . . . . . 28

2.9 Next steps . . . . . . . . . . . . . . . . . 29

3 Branching Ratio Measurements of $D^{0} \rightarrow \pi^{-} \pi^{+} \pi^{0}, K^{-} K^{+} \pi^{0} \quad 30$

3.1 Description of backgrounds . . . . . . . . . . . . . . . 30

3.2 Extracting signal events . . . . . . . . . . . . . . . . . . . . . . . 31

3.3 Signal reconstruction efficiency . . . . . . . . . . . . . . 36

3.4 Number of signal events produced . . . . . . . . . . . . . 38

3.5 Calculation of ratios of branching ratios . . . . . . . . . . 38

3.6 Study of systematic uncertainties . . . . . . . . . . . . . . . . . 38

3.7 Systematic error in average weight . . . . . . . . . . . . . . . . . 40

3.7.1 Systematic error due to simulation statistics . . . . . . . . . . . 40

3.7.2 Systematic error from background subtraction . . . . . . . 41

$3.8 \Delta m$ systematics . . . . . . . . . . . . . . . . . . 45 
3.9 Effect of background model on signal yield . . . . . . . . . . . . . 45

3.10 Effect of difference in $P^{*}$ distributions in data and simulation . . . . 45

3.11 Particle-identification correction and systematics . . . . . . . . . 46

3.12 Tracking efficiency systematics . . . . . . . . . . . . . . 46

3.13 Removal of $D^{0} \rightarrow K_{S}^{0} \pi^{0}$ from $D^{0} \rightarrow \pi^{-} \pi^{+} \pi^{0}$ events . . . . . . . . . 47

3.14 Summary of corrections . . . . . . . . . . . . . . . . 48

3.15 Summary of systematic uncertainties . . . . . . . . . . . . . 48

3.16 Consistency check: analysis on disjoint data sub-samples . . . . . . . 48

3.17 Results and conclusions . . . . . . . . . . . . . . . . . . 49

4 Amplitude Analysis of the Decay $D^{0} \rightarrow K^{-} K^{+} \pi^{0} \quad \mathbf{5 3}$

4.1 Introduction . . . . . . . . . . . . . . . 53

4.2 Event selection . . . . . . . . . . . . . . . . . 54

4.3 Efficiency calculation . . . . . . . . . . . . . . . 54

4.4 Parametrization of amplitudes . . . . . . . . . . . . 55

4.5 Parametrization of $P$ - and $D$ - wave amplitudes $\ldots \ldots \ldots$

4.6 Parametrization of $K^{ \pm} \pi^{0} S$-wave amplitudes . . . . . . . . . . . 56

4.7 Nature of $K^{ \pm} \pi^{0} S$-wave . . . . . . . . . . . . . . . . 56

4.8 Parametrization of $K^{-} K^{+} S$-wave amplitude . . . . . . . . . 57

4.9 Maximum likelihood fit to data . . . . . . . . . . . . . . 57

4.10 Result on $K \pi$ S-wave . . . . . . . . . . . . . . . . . . . . . 62

4.11 Results of the Dalitz plot fit . . . . . . . . . . . . . 62

4.12 Are there additional states ?. . . . . . . . . . . . . 62

4.13 Description of angular moments . . . . . . . . . . . . . . 62

4.14 Model-independent partial wave analysis . . . . . . . . . . . . . 64

4.15 Systematic uncertainties . . . . . . . . . . . . . . 66

4.16 Strong-phase difference and suppression factor . . . . . . . . . 66

4.17 Summary . . . . . . . . . . . . . . . . . . 66

5 Amplitude Analysis of the Decay $D^{0} \rightarrow \pi^{-} \pi^{+} \pi^{0} \quad 68$

5.1 Data and simulation samples . . . . . . . . . . . . . 68

5.2 Event selection . . . . . . . . . . . . . . . . . . 68

5.3 Efficiency calculation . . . . . . . . . . . . . . . . . . 69

5.4 Background model for Dalitz plot . . . . . . . . . . . . . 69

5.5 Fitting simulated events . . . . . . . . . . . . . . 70

5.6 Dalitz plot fit for data . . . . . . . . . . . . . . 70

5.7 Parameter-correlation matrix . . . . . . . . . . . . 72

5.8 Fit with CLEO parametrization . . . . . . . . . . . . 77

5.9 Effort to include $p$-wave nonresonant terms . . . . . . . . . . . . 79

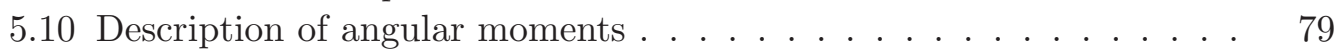

5.11 Systematic uncertainties . . . . . . . . . . . . . . . 80

5.12 Strong-phase difference and suppression factor . . . . . . . . . 82

5.13 Summary . . . . . . . . . . . . . . . . . . 82 
6 Measurement of $\gamma$ Using $B^{ \pm} \rightarrow D_{\pi^{+} \pi^{-} \pi^{0}} K^{ \pm} \quad 83$

6.1 Introduction . . . . . . . . . . . . . . . . . . 84

6.2 Detector and dataset . . . . . . . . . . . . . . . . . . . 84

6.3 Event selection . . . . . . . . . . . . . . . . . . 84

6.4 Signal-background discrimination . . . . . . . . . . . . 85

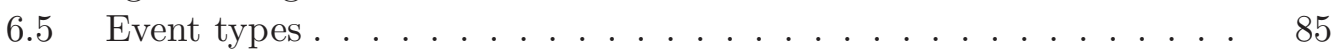

6.6 Analysis procedure . . . . . . . . . . . . . . 85

6.7 Step 1: $\boldsymbol{D}^{\mathbf{0}}$ Dalitz plot fit using $\mathrm{D}^{*}$ sample . . . . . . . . . . . 86

6.8 Next Steps . . . . . . . . . . . . . . . . . . . . . 86

6.9 Signal yields and asymmetry . . . . . . . . . . . . . . 88

6.10 Likelihood function . . . . . . . . . . . . . . . . . . . . . . . 88

6.11 Polar coordinates . . . . . . . . . . . . . . . . . . 88

6.12 Systematics . . . . . . . . . . . . . . . . . . 89

6.13 Validation ............................ 89

6.14 Results in terms of experimental parameters . . . . . . . . . . . . 91

6.15 Constraints on physical parameters . . . . . . . . . . . . . . 91

7 Search for $C P$ Violation in Charm Decays $\quad 93$

7.1 Motivation ......................... 93

7.2 Previous experimental studies . . . . . . . . . . . . . . . . 94

7.3 Why three-body decays $\quad \ldots \ldots \ldots$. . . . . . . . . . . 95

7.4 Event selection . . . . . . . . . . . . . . . . . 95

7.5 Signal reconstruction efficiency . . . . . . . . . . . . . . 96

7.6 Sensitivity study with simulated data . . . . . . . . . . . . . . 97

7.6.1 Asymmetry in two-dimensional Dalitz plot . . . . . . . . . 97

7.6.2 Asymmetry in distribution of angular moments . . . . . . . . 99

$7.6 .3 \quad \chi^{2} / \nu$ from Dalitz plot distribution . . . . . . . . . . . . 99

$7.6 .4 \chi^{2} / \nu$ from angular moment distribution . . . . . . . . . . 102

7.6.5 Correlation among the angular moments . . . . . . . . . . 102

7.6.6 Model dependent analysis on simulated data . . . . . . . . 103

7.7 Systematic uncertainty in simulated data . . . . . . . . . . . . . 110

7.7.1 Experimental and model-dependent systematics . . . . . . . . 110

7.7.2 Systematic uncertainty from $D^{0} / \bar{D}^{0}$ mistagging . . . . . . . . 110

7.8 Validation study using simulation treated as data . . . . . . . . . . . 111

7.8.1 Analysis with $C P$-symmetric $D \rightarrow \pi^{-} \pi^{+} \pi^{0}$ simulated events . 111

7.8.2 Analysis with asymmetric $D \rightarrow \pi^{-} \pi^{+} \pi^{0}$ simulated events . . 112

7.8.3 Analysis with $C P$-symmetric $D \rightarrow K^{-} K^{+} \pi^{0}$ simulated events 112

7.8.4 Analysis with asymmetric $D \rightarrow K^{-} K^{+} \pi^{0}$ simulated events . 113

7.8.5 Validation of the model-independent method on data . . . . . 113

7.8.6 Validation on full available simulation sample . . . . . . . . . 113

7.9 Asymmetry in Dalitz plot . . . . . . . . . . . . . . . . . . 115

7.10 Asymmetry in branching ratio . . . . . . . . . . . . . . . . . 116

7.11 Steps for unblinding . . . . . . . . . . . . . . . 116

7.12 Results from data . . . . . . . . . . . . . . . . 118

7.12.1 Asymmetry in Dalitz plot distribution . . . . . . . . . . . 118

7.12.2 Asymmetry in angular moments distribution . . . . . . . . . 118 
7.12 .3 Results of model fit . . . . . . . . . . . . . . . . . . . 123

7.12 .4 Systematic uncertainties . . . . . . . . . . . . . . . . 124

7.12.5 Asymmetry in branching ratio vs asymmetry in Dalitz Plot . 124

7.13 Summary . . . . . . . . . . . . . . . . 125 


\section{List of Figures}

1.1 Examples of (a) Cabibbo-favored, (b) singly Cabibbo-suppressed, and (c) doubly Cabibbo-suppressed decays. The weak vertices shown with open circles are Cabibbo-favored while those shown with filled circles are Cabibbo-suppressed. . . . . . . . . . . . . . . . 15

1.2 Hadronic and semi-leptonic decay of the charm quark. . . . . . . . . 16

1.3 Three types of tree-level Feynman diagrams for the decay $D^{0} \rightarrow$ $\pi^{-} \pi^{+} \pi^{0} \ldots \ldots \ldots \ldots \ldots 16 \ldots \ldots \ldots$

1.4 Three types of tree-level Feynman diagrams for the decay $D^{0} \rightarrow$

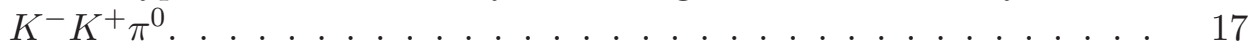

1.5 Diagrams contributing to $B^{-} \rightarrow \tilde{D}^{0} K^{-}$decay. The left diagram proceeds via $b \rightarrow c \bar{u} s$ transition, while the right diagram proceeds via $b \rightarrow u \bar{c} s$ transition and is color suppressed. . . . . . . . . . . 18

2.1 BABAR detector longitudinal section (source Ref. [12]). . . . . . . . . 21

2.2 BABAR detector end view (source Ref. [12]). . . . . . . . . . . . . 21

2.3 Kaon identification efficiency (top) and pion mis-identification rate (bottom) as a function of track momentum for positive (left) and negative (middle) tracks. The rightmost plots show ratios of efficiencies in data and simulation. . . . . . . . . . . . . . . . . .

2.4 Pion identification efficiency (top) and kaon mis-identification rate (bottom) as a function of track momentum for negative (left) and positive (middle) tracks. The rightmost plots show ratios of efficiencies in data and simulation. . . . . . . . . . . . . . .

3.1 $\Delta m=m_{D^{*}}-m_{D^{0}}$ distributions for $K^{-} \pi^{+} \pi^{0}$ (top), $\pi^{-} \pi^{+} \pi^{0}$ (middle), and $K^{-} K^{+} \pi^{0}$ (bottom) generic $c \bar{c}$ simulated events around $\pm 1 \sigma$ of the $D^{0}$ invariant mass. . . . . . . . . . . . . . . . . .

$3.2 \Delta m=m_{D^{*}}-m_{D^{0}}$ distributions for $K^{-} \pi^{+} \pi^{0}$ (top), $\pi^{-} \pi^{+} \pi^{0}$ (middle), and $K^{-} K^{+} \pi^{0}$ (bottom) data events around $\pm 1 \sigma$ of the $D^{0}$ invariant mass. . . . . . . . . . . . . . . . .

3.3 Simulated $\pi^{-} \pi^{+} \pi^{0}$ (left) and $K^{-} K^{+} \pi^{0}$ (right) invariant mass distributions. Signal events are shown as open histograms. Combinatorial and reflection backgrounds are shown by the light and dark shaded histograms, respectively. The signal region is delimited by the vertical lines. . . . . . . . . . . . . . . . . .

3.4 Simulated $K^{-} \pi^{+} \pi^{0}$ events reconstructed as $\pi^{-} \pi^{+} \pi^{0}$ (left) and the level of this reflection in $\pi^{-} \pi^{+} \pi^{0}$ data sample (right). . . . . . . . . 
3.5 Simulated $K^{-} \pi^{+} \pi^{0}$ events reconstructed as $K^{-} K^{+} \pi^{0}$ (left) and the level of this reflection in $K^{-} K^{+} \pi^{0}$ data sample (right). . . . . . .

3.6 Fitted mass for the $K^{-} \pi^{+} \pi^{0}, \pi^{-} \pi^{+} \pi^{0}$, and $K^{-} K^{+} \pi^{0}$ data samples. Dots are data points and the solid curves are the fit. The dot-dashed lines show the level of combinatorial background in each case. For the $\pi^{-} \pi^{+} \pi^{0}$ and the $K^{-} K^{+} \pi^{0}$ modes, the shaded region represents the total background. . . . . . . . . . . . . . . .

3.7 Efficiency across Dalitz plots for $K^{-} \pi^{+} \pi^{0}, \pi^{-} \pi^{+} \pi^{0}$, and $K^{-} K^{+} \pi^{0}$ signal reconstruction obtained using simulated events from $c \bar{c}$ decays, generated uniformly in the available phase space. The observed efficienciencies are shown on the left and the parametrized ones (using a 3rd order polynomial) are shown on the right. . . . . . . . . . . .

3.8 Efficiency corrected $K^{-} \pi^{+} \pi^{0}, \pi^{-} \pi^{+} \pi^{0}$, and $K^{-} K^{+} \pi^{0}$ Dalitz plots. .

3.9 Comparison of $D^{0} \rightarrow \pi^{-} \pi^{+} \pi^{0}$ Dalitz plot distributions for events in the upper sideband of $m_{D^{0}}$ in data and simulation. . . . . . . . .

3.10 Comparison of $D^{0} \rightarrow K^{-} K^{+} \pi^{0}$ Dalitz plot distributions for events in the lower sideband of $m_{D^{0}}$ in data and simulation. . . . . . . . .

3.11 Comparison of $D^{0} \rightarrow \pi^{-} \pi^{+} \pi^{0}$ Dalitz plot distributions for events in the signal region and in the upper sideband of $m_{D^{0}}$ in simulation. . .

3.12 Comparison of $D^{0} \rightarrow K^{-} K^{+} \pi^{0}$ Dalitz plot distributions for events in the signal region and in the lower sideband of $m_{D^{0}}$ in simulation. . .

3.13 Comparison of the ratio of particle-identification rates in data and simulation for our track selection with those from the standard calibration. . . . . . . . . . . . . . . .

3.14 Fit for $K_{S}^{0}$ peak in the $\pi^{-} \pi^{+}$invariant mass plot (top) and the distribution of signed distance of the $\pi^{-} \pi^{+}$vertex from the beam spot

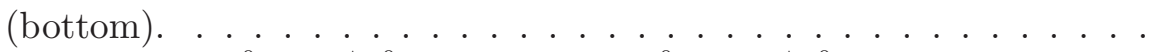

3.15 Measured $\frac{\mathcal{B}\left(D^{0} \rightarrow \pi^{-} \pi^{+} \pi^{0}\right)}{\mathcal{B}\left(D^{0} \rightarrow K^{-} \pi^{+} \pi^{0}\right)}$ (left) and $\frac{\mathcal{B}\left(D^{0} \rightarrow K^{-} K^{+} \pi^{0}\right)}{\mathcal{B}\left(D^{0} \rightarrow K^{-} \pi^{+} \pi^{0}\right)}$ (right) of disjoint data samples. The horizontal red line shows the result for the full data sample. Errors are statistical only. . . . . . . . . . . . 49

3.16 Dalitz boundaries . . . . . . . . . . . . . . . 52

4.1 Dalitz plot for $D^{0} \rightarrow K^{-} K^{+} \pi^{0}$ data (a), and the corresponding squared invariant mass projections $(\mathrm{b}-\mathrm{d})$. The three-body invariant mass of the $D^{0}$ candidate is constrained to the nominal value. In plots (b-d), the dots (with error bars, black) are data points and the solid lines (blue) correspond to the best isobar fit models. . . . . . .

4.2 LASS (solid line, blue) and E-791 (dots with error bars) $K \pi S$-wave amplitudes (a), in arbitrary units, and phase (b). The double headed arrow (red) indicates the mass range available in the decay $D^{0} \rightarrow$

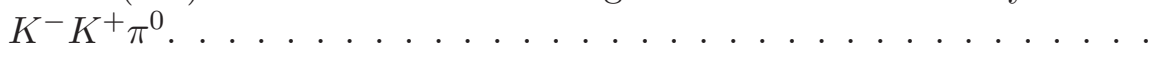


4.3 The phase-space-corrected $K^{-} K^{+} S$ - and $P$-wave amplitudes, $|S|$ and $|P|$ respectively, in arbitrary units, as functions of the invariant mass. (a) Lineshapes for (solid line, blue) $f_{0}(980)$, and (broken line, blue) $a_{0}(980)$, derived from Eq. 4.8. (b) Lineshape for $\phi(1020)$ (solid line, blue). In each plot, solid circles with error bars correspond to values obtained from the model-independent analysis for $|S|$ and $|P|$ using Eq. 4.9. In (a), the open triangles (red) correspond to values obtained from the decay $D^{0} \rightarrow K^{-} K^{+} \bar{K}^{0}$ (see text). . . . . . . . . .

4.4 The mass dependence of the spherical harmonic moments of $\cos \theta_{H}$ after efficiency corrections and background subtraction: $K^{+} \pi^{0}$ (top) and $K^{-} K^{+}$(bottom). The circles with error bars are data points and the curves (red) are derived from the fit functions (see text). For the sake of visibility, we do not show error bars on the curves. . . . . . .

4.5 Results of the partial-wave analysis of the $K^{-} K^{+}$system using Eq. 4.9 described in the text. (a) Cosine of relative phase $\theta_{S P}=\theta_{S}-\theta_{P}$, (b) two solutions for $\theta_{S P}$, (c) $P$-wave phase taken from Eqs. 4.4-4.5 for the $\phi(1020)$ meson, and (d) $S$-wave phase derived from the upper solution in (b). Solid bullets are data points, and open circles (blue) and open triangles (red) correspond, respectively, to isobar models I and II. The number of simulated events used for the two models is 10 times larger than data. Errors for quantities from the isobar models arise from Monte Carlo statistical limitations, and differ from errors derived from Eq. 4.9. . . . . . . . . . . . . . . .

5.1 (Row 1) Invariant-mass projections for $\pi^{-} \pi^{+} \pi^{0}$ simulated events generated with CLEO parameters of Ref. [41] and fitted with a sum of three Breit-Wigners for $\rho(770)$ states. (Rows 2-3) Pull distributions for the fit parameters. . . . . . . . . . . . . . . .

$5.2 \pi^{-} \pi^{+} \pi^{0}$ Dalitz plot for events generated with CLEO parameters and fitted with a sum of three Breit-Wigner amplitudes for the three $\rho(770)$ states. Background generated and fitted with a shape taken from Data sideband. . . . . . . . . . . . . . . . .

5.3 Efficiency-corrected Dalitz plot and invariant mass-squared projections for the $D^{0} / \bar{D}^{0} \rightarrow \pi^{-} \pi^{+} \pi^{0}$ decay excluding $D^{0} / \bar{D}^{0} \rightarrow K_{s}^{0} \pi^{0}$. .

5.4 Invariant mass projections (top row), Dalitz plot (second row), and normalized residuals (bottom row) for the fit with only $\rho(770)$ and a nonresonant term. . . . . . . . . . . . . .

5.5 The mass dependence of the spherical harmonic moments of $\cos \theta_{H}$ after efficiency corrections and background subtraction: $\pi^{+} \pi^{0}$ (top) and $\pi^{-} \pi^{+}$(bottom) channels of the $D^{0} / \bar{D}^{0} \rightarrow \pi^{-} \pi^{+} \pi^{0}$ decay. The circles with error-bars are data points and the curves (red) are derived from the fit parametrization of Table 5.1. For the sake of visibility, we do not show error bars on the curves. . . . . . . . . . . . 
6.1 (Charge conjugation is implied for all plots.) (a,b) Projections of the $D^{*+} \rightarrow D^{0} \pi^{+}$data events and PDF onto the Dalitz plot variables $s_{+}$ and $s_{-}$. (c) The 2-dimensional $\left(s_{+}, s_{-}\right)$distribution of the $D^{*+} \rightarrow$ $D^{0} \pi^{+}$data. (d) One-, two-, and three-standard-deviation contours of $\mathcal{L}$ as a function of $\theta_{ \pm}$vs. $\rho_{ \pm}$. The solid (dashed) curves correspond to $B^{+}\left(B^{-}\right)$results. The no-interference point $\left(\rho_{ \pm}=x_{0}, \theta_{ \pm}=180^{\circ}\right)$ is marked with an $\times$. (e,f) Projection of the $B^{-} \rightarrow D_{\pi^{+} \pi^{-} \pi^{0}} K^{-}$ candidate data onto $s_{+}$and $s_{-} \ldots \ldots \ldots \ldots$

6.2 (a) Projection of the three-dimensional confidence level, $1-\alpha$, onto $r_{B}$ and $\gamma$. (b) confidence level, $1-\alpha$, versus $\gamma \ldots \ldots$

7.1 The difference between $D^{0}$ and $\bar{D}^{0}$ Dalitz plot distributions, in units of $\sigma$, for decays to the $\pi^{-} \pi^{+} \pi^{0}$ (left) and $K^{-} K^{+} \pi^{0}$ (right) final states. The amplitudes and phases for $D^{0}$ and $\bar{D}^{0}$ events are equal. The size of the simulated sample used here is roughly 25 times larger than our data sample. . . . . . . . . . . . . . . .

7.2 Difference between $D^{0}$ and $\bar{D}^{0}$ Dalitz plot distributions, in units of $\sigma$, for the $\pi^{-} \pi^{+} \pi^{0}$ (top) and $K^{-} K^{+} \pi^{0}$ (bottom) decays. In the top left (right) plot $\bar{D}^{0} \rightarrow \rho^{0} \pi^{0}\left(\bar{D}^{0} \rightarrow \rho^{-} \pi^{+}\right)$amplitude has been changed by $+5 \%$, and its phase has been changed by $+5^{\circ}$. In the bottom left (right) plot $\bar{D}^{0} \rightarrow \phi \pi^{0}\left(\bar{D}^{0} \rightarrow K^{*}(892)^{-} K^{+}\right)$amplitude has been changed by $+5 \%$, and its phase has been changed by $+5^{\circ}$. The size of the simulated sample used here is $\sim 25$ times larger than our data sample. . . . . . . . . . . . . . . .

7.3 The difference in $\pi^{-} \pi^{+}$channel Legendre polynomial moments between $D^{0}$ and $\bar{D}^{0}$ decays to the $\pi^{-} \pi^{+} \pi^{0}$ final state (in number of $\sigma$ 's). The top two rows correspond to the case of no asymmetry. In the bottom two rows, the $\bar{D}^{0} \rightarrow \rho^{0} \pi^{0}$ amplitude has been changed by $+5 \%$, and its phase has been changed by $+5^{\circ}$. The size of the simulated sample roughly corresponds to the size of our data sample.

7.4 The difference in $\pi^{+} \pi^{0}$ channel Legendre polynomial moments between $D^{0}$ and $\bar{D}^{0}$ decays to the $\pi^{-} \pi^{+} \pi^{0}$ final state (in number of $\sigma$ 's). The top two rows correspond to the case of no asymmetry. In the bottom two rows, the $\bar{D}^{0} \rightarrow \rho^{-} \pi^{+}$amplitude has been changed by $+5 \%$, and its phase has been changed by $+5^{\circ}$. The size of the simulated sample roughly corresponds to the size of our data sample.

7.5 The difference in $K^{-} K^{+}$channel Legendre polynomial moments between $D^{0}$ and $\bar{D}^{0}$ decays to the $K^{-} K^{+} \pi^{0}$ final state (in number of $\sigma^{\prime}$ 's). The top two rows correspond to the case of no asymmetry. In the bottom two rows, the $\bar{D}^{0} \rightarrow \phi \pi^{0}$ amplitude has been changed by $+5 \%$, and its phase has been changed by $+5^{\circ}$. The size of the simulated sample roughly corresponds to the size of our data sample. 
7.6 The difference in $K^{+} \pi^{0}$ channel Legendre polynomial moments between $D^{0}$ and $\bar{D}^{0}$ decays to the $K^{-} K^{+} \pi^{0}$ final state (in number of $\sigma^{\prime}$ 's). The top two rows correspond to the case of no asymmetry. In the bottom two rows, the $\bar{D}^{0} \rightarrow K^{*}(892)^{-} K^{+}$amplitude has been changed by $-5 \%$, and its phase has been changed by $-5^{\circ}$. The size of the simulated sample corresponds to the size of our data sample. . .

$7.7 \chi^{2} / \nu$ for difference in angular moments in case of no $C P$ asymmetry obtained from simulation: $\pi^{+} \pi^{-}$channel of $D \rightarrow \pi^{-} \pi^{+} \pi^{0}$ (left), $K^{+} K^{-}$channel of $D \rightarrow K^{-} K^{+} \pi^{0}$ (right). . . . . . . . . . .

$7.8 \chi^{2} / \nu$ for difference in angular moments in case of no $C P$ asymmetry obtained from simulation: $\pi^{+} \pi^{0}$ channel of $D \rightarrow \pi^{-} \pi^{+} \pi^{0}$ (left), $K^{+} \pi^{0}$ channel of $D \rightarrow K^{-} K^{+} \pi^{0}$ (right). . . . . . . . . . . . . . 105

$7.9 \chi^{2} / \nu$ for difference in Dalitz plot structure in case of no $C P$ asymmetry obtained from simulation: $D \rightarrow \pi^{-} \pi^{+} \pi^{0}$ (left), $D \rightarrow K^{-} K^{+} \pi^{0}$ (right). 105

7.10 The spread of $\chi^{2} / \nu$ values corresponding to the difference in angular moments distributions between two catagories of randomly chosen data events: $\pi^{+} \pi^{-}$(left) and $\pi^{+} \pi^{0}$ (right). . . . . . . . . . . . 114

7.11 Relative $\pi_{s}$ efficiency map (left), $\frac{\epsilon_{\pi_{s}^{+}}}{\epsilon_{\pi_{s}^{-}}}$, with statistical errors (right). . 117

7.12 Dalitz plots for $D^{0}$ (left) and $\bar{D}^{0}$ (right) events in the $\widetilde{D} \rightarrow \pi^{-} \pi^{+} \pi^{0}$ decay. . . . . . . . . . . . . . . . . . . 119

7.13 Dalitz plots for $D^{0}$ (left) and $\bar{D}^{0}$ (right) events in the $\widetilde{D} \rightarrow K^{-} K^{+} \pi^{0}$ decay. . . . . . . . . . . . . . . . . . . . . . 119

7.14 Difference between the $\bar{D}^{0}$ and $D^{0}$ Dalitz plots for $\widetilde{D} \rightarrow \pi^{-} \pi^{+} \pi^{0}$ (left) and $\widetilde{D} \rightarrow K^{-} K^{+} \pi^{0}$ (right). . . . . . . . . . . . . . .

7.15 Normalized residuals in Dalitz plot elements, defined in Eq. 7.11, for (a) $\widetilde{D} \rightarrow \pi^{-} \pi^{+} \pi^{0}$ and (b) $\widetilde{D} \rightarrow K^{-} K^{+} \pi^{0} \ldots \ldots \ldots \ldots$

7.16 Normalized residuals for the first three Legendre polynomial moments of the $\pi^{-} \pi^{+}$(row 1 ), $\pi^{+} \pi^{0}$ (row 2), $K^{-} K^{+}$(row 3 ), and $K^{+} \pi^{0}$ (row 4) sub-systems. The confidence level for no $C P$ violation (dashed line) is obtained from the first eight moments. The error bars represent $\pm 1 \sigma .121$

7.17 Phase-space-integrated $C P$ asymmetry as a function of the cosine of the polar angle of the reconstructed $\widetilde{D}$ candidate CM momentum for (a) $\widetilde{D} \rightarrow \pi^{-} \pi^{+} \pi^{0}$ and (b) $\widetilde{D} \rightarrow K^{-} K^{+} \pi^{0}$ decays. The dashed lines represent the central values, and the shaded regions the $1 \sigma$ intervals. 


\section{List of Tables}

2.1 PEP-II beam parameter values for a typical colliding beam operation. HER and LER refer to the high energy $e^{-}$and low energy $e^{+}$ring, respectively. $\sigma_{L x}, \sigma_{L y}$, and $\sigma_{L z}$ refer to the horizontal, vertical, and longitudinal $\mathrm{rms}$ size of the luminous region. . . . . . . . . .

2.2 The basic track selection criteria used for charged particles. $\left|x_{0}\right|$ is the absolute value of the closet distance between the track and the beam spot in the $x-y$ plane. . . . . . . . . . . . . . . .

2.3 Charged particle identification requirements. The tracks are also required to be inconsistent with electron hypothesis if their momentum is $>0.4 \mathrm{GeV} /$ c . . . . . . . . . . . . . . . .

2.4 Mean and rms values of $\Delta m$ distribution for reconstructed $D^{0}$ candidates having mass within $1 \sigma$ of the central value. . . . . . . . 28

2.5 Event selection criteria . . . . . . . . . . . . . . . 28

2.6 Efficiency after successive selection requirements. . . . . . . . . . . 29

3.1 Mean and RMS values of $D^{0}$ candidate invariant mass distribution with both signal and some background. . . . . . . . . . . 36

3.2 A summary of the number of observed events and other fit results for the reconstructed $D^{0}$ invariant mass. . . . . . . . . . . . 36

3.3 Final yield and average weight and errors associated with them. . . . 38

3.4 Results for different $\Delta m$ cuts. . . . . . . . . . . . . . . . . 45

3.5 Summary of corrections . . . . . . . . . . . . . . . . 48

3.6 Summary of systematic uncertainties . . . . . . . . . . . . . . . . 49

3.7 Measurements of disjoint samples. . . . . . . . . . . . . 50

3.8 A comparison of the previous PDG [20] results with this analysis. . . 51

3.9 Areas covered by Dalitz plots. . . . . . . . . . . . . . . 52

4.1 The results obtained from the $D^{0} \rightarrow K^{-} K^{+} \pi^{0}$ Dalitz plot fit. We define amplitude coefficients $a_{r}$ and $\phi_{r}$ relative to those of the $K^{*}(892)^{+}$. The errors are statistical and systematic, respectively. We show the $a_{0}(980)$ contribution, when it is included in place of the $f_{0}(980)$, in square brackets. We denote the $K \pi S$-wave states here by $K^{ \pm} \pi^{0}(S)$. We use LASS amplitude to describe the $K \pi S$-wave states in both the isobar models (I and II). . . . . . . . . . . . . . . . . . . . . 59

4.2 Parameter index for the correlation matrix. . . . . . . . . . . . 59 
4.3 Parameter correlation matrix for fit Model-I. The parameter indices are explained in Table $4.2 . \ldots \ldots \ldots$. . . . . . . . .

4.4 Parameter correlation matrix for fit Model-II. Parameters with indices 1-4 of Table 4.2 are not included in this fit model. . . . . . . . . . .

5.1 Result of the fit to the $D^{0} \rightarrow \pi^{-} \pi^{+} \pi^{0}$ Dalitz plot distribution. The amplitudes and phases are defined relative to those of the $\rho^{+}$. The fraction is $f_{r} \equiv \int\left|a_{r} A_{r}\right|^{2} d s_{-} d s_{+} / \int\left|f_{D^{0}}\right|^{2} d s_{-} d s_{+}$. The errors are statistical and systematic, respectively. We take the mass (width) of the $\sigma$ meson to be $400(600) \mathrm{MeV} / \mathrm{c}^{2}$. The $\chi^{2} / \nu$ for the fit is 1.38 for

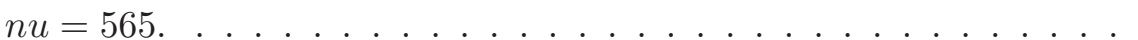

5.2 Parameter index for the correlation matrix. . . . . . . . . . . . . 72

5.3 Result of the fit to the $D^{0} \rightarrow \pi^{-} \pi^{+} \pi^{0}$ Dalitz plot with only $\rho(770)$ states and a nonresonant term. The errors are statistical only. The $\chi^{2} / \nu$ for the fit is 2.82 for $\nu=574 \ldots \ldots \ldots \ldots$. . . . . . .

5.4 Parameters of the nonresonant p-wave found in the data fit, and the corresponding fit fractions (not in percent!) of these components for a PDF that contains only these components with the parameters shown.

6.1 Result of the fit to the $D^{*+} \rightarrow D^{0} \pi^{+}$sample, showing the amplitudes ratio $R_{r} \equiv a_{r} / a_{\rho^{+}(770)}$, phase differences $\Delta \phi_{r} \equiv \phi_{r}-\phi_{\rho^{+}(770)}$, and fit fractions $f_{r} \equiv \int\left|a_{r} A_{r}\left(s_{+}, s_{-}\right)\right|^{2} d s_{-} d s_{+}$. The first (second) errors are statistical (systematic). We take the mass (width) of the $\sigma$ meson to be $400(600) \mathrm{MeV} / c^{2} \ldots \ldots \ldots \ldots \ldots$

7.1 Experimental measurements of direct $C P$ asymmetry in $D^{0}-\bar{D}^{0}$ decays (source: Ref. [20]). . . . . . . . . . . . . . . . .

7.2 Fit results for the efficiency coefficients of Eq. (7.3) for the signal reconstruction efficiency. . . . . . . . . . . . . . .

7.3 Model-independent $\chi^{2} / \nu$ values obtained from the difference in Dalitz plots of $D^{0}$ and $\bar{D}^{0}$ events for different levels of asymmetry in one of the intermediate states of the simulated $D^{0} \rightarrow \pi^{-} \pi^{+} \pi^{0}(\nu=1429$, size $\left.675 \mathrm{fb}^{-1}\right)$ and $D^{0} \rightarrow K^{-} K^{+} \pi^{0}\left(\nu=726\right.$, size $\left.440 \mathrm{fb}^{-1}\right)$ samples.

7.4 Model-independent $\chi^{2} / \nu$ (where $\nu=320$ ) values obtained from the difference in Legendre polynomial moments of $D^{0}$ and $\bar{D}^{0}$ events for different levels of asymmetry in one of the amplitudes $\left(\rho^{+}\right.$or $\left.\rho^{0}\right)$ of the simulated $D^{0} \rightarrow \pi^{-} \pi^{+} \pi^{0}$ sample of size $675 \mathrm{fb}^{-1}$. The amplitude ratios are in percent and the angles are in degrees. . . . . . . . . .

7.5 Model-independent $\chi^{2} / \nu$ (where $\nu=160$ ) values obtained from the difference in Legendre polynomial moments of $D^{0}$ and $\bar{D}^{0}$ events for different levels of asymmetry in one of the amplitudes $\left(K^{*}(892)^{+}\right.$or $\phi(1020))$ of the simulated $D^{0} \rightarrow K^{-} K^{+} \pi^{0}$ sample of size $440 \mathrm{fb}^{-1}$. The amplitude ratios are in percent and the angles are in degrees. 
7.6 Model-dependent asymmetry values obtained from the likelihood fit for different levels of generated asymmetry in the $D^{0} \rightarrow \rho^{0} \pi^{0}$ state in the Monte Carlo $D^{0} \rightarrow \pi^{-} \pi^{+} \pi^{0}$ sample of size $675 \mathrm{fb}^{-1}$. The amplitude ratios are in percent and the angles are in degrees. . . . .

7.7 Model-dependent asymmetry values obtained from the likelihood fit for different levels of generated asymmetry in the $D^{0} \rightarrow \rho^{+} \pi^{-}$state in the Monte Carlo $D^{0} \rightarrow \pi^{-} \pi^{+} \pi^{0}$ sample of size $675 \mathrm{fb}^{-1}$. The amplitude ratios are in percent and the angles are in degrees. . . . . 107

7.8 Model-dependent asymmetry values obtained from the likelihood fit for different levels of generated asymmetry in the $D^{0} \rightarrow \phi(1020) \pi^{0}$ state in the Monte Carlo $D^{0} \rightarrow K^{-} K^{+} \pi^{0}$ sample of size $440 \mathrm{fb}^{-1}$. The amplitude ratios are in percent and the angles are in degrees.

7.9 Model-dependent asymmetry values obtained from the likelihood fit for different levels of generated asymmetry in the $D^{0} \rightarrow K^{*}(892)^{+} K^{-}$ state in the Monte Carlo $D^{0} \rightarrow K^{-} K^{+} \pi^{0}$ sample of size $440 \mathrm{fb}^{-1}$. The amplitude ratios are in percent and the angles are in degrees.

7.10 Observed asymmetry in amplitude and phase and the $\chi^{2} /$ dof values for different levels of cross-feed. In the model-dependent fit, the amplitude and phase coefficients are determined using $D^{0} \rightarrow \rho^{+} \pi^{-}$(for $D^{0} \rightarrow \pi^{-} \pi^{+} \pi^{0}$ ) and $D^{0} \rightarrow K^{*+} K^{-}$(for $D^{0} \rightarrow K^{-} K^{+} \pi^{0}$ ) decay. . . .

7.11 The values and spread of $\chi^{2} / \nu$ for the null hypothesis (no $C P$ violation) in the two model-independent analysis methods. These were obtained using 500 simulation experiments. See Fig. 7.7 (for angular moments distributions) and Fig. 7.9 (for Dalitz plot distributions) for further details. . . . . . . . . . . . . . . .

7.12 The results obtained from the model-dependent analysis of $D^{0} / \bar{D}^{0} \rightarrow$ $\pi^{-} \pi^{+} \pi^{0}$ Dalitz plot. We define amplitude coefficients, $a_{r}$ and $\phi_{r}$, relative to those of the $\rho(770)^{+}$. The errors are statistical. . . . . . .

7.13 The results obtained from the model-dependent analysis of $D^{0} / \bar{D}^{0} \rightarrow$ $K^{-} K^{+} \pi^{0}$ Dalitz plot. We define amplitude coefficients, $a_{r}$ and $\phi_{r}$, relative to those of the $K^{*}(892)^{+}$. The errors are statistical. We use Model-I of Ref. [21] here. . . . . . . . . . . . . . . . . .

7.14 Model-dependent $C P$ asymmetry in the $\widetilde{D} \rightarrow \pi^{-} \pi^{+} \pi^{0}$ Dalitz plots. The first and second errors are statistical and systematic, respectively. For details on the Dalitz plot parametrization and the $a_{r}, \phi_{r}$, and $f_{r}$ values, see Ref. [25]. . . . . . . . . . . . . . . .

7.15 Model-dependent $C P$ asymmetry in the $\widetilde{D} \rightarrow K^{-} K^{+} \pi^{0}$ Dalitz plots. The errors are statistical and systematic, respectively. We show the $a_{0}(980)$ contribution, when it is included in place of the $f_{0}(980)$, in square brackets. For details on the Dalitz plot parametrization and the $a_{r}, \phi_{r}$, and $f_{r}$ values, see Ref. [21]. We use Model-I of Ref. [21] to obtain central values and Model-II for study of systematic errors. . . 


\section{Chapter 1}

\section{Introduction}

\subsection{Standard Model}

The Standard Model (SM) [1] of particle physics is the most accepted theory for describing the interactions between the elementary particles that form the building blocks of the universe. It says that everything around us is made of fundamental particles called quarks and leptons, with three types of fundamental forces acting on them through "carrier particles". It describes these forces - electromagnetic, weak nuclear, and strong nuclear - in a unified way. It has, so far, been able to account for almost all observed experimental phenomena.

Despite its incredible success, the SM has a few serious deficiencies. For example, it does not describe the fourth fundamental force of natute, the gravity, at all. Also, now we know that 96 percent of the universe is not made of matter, which does not fit well into the SM.

\subsection{Weak interaction}

The weak nuclear force (also called the "weak force") is one of the fundamental interactions described by the SM. In general, it can act between two leptons, between a lepton and a hadron, or between two hadrons. It was first observed and studied in the $\beta$ decay,

$$
n \rightarrow p+e^{-}+\overline{\nu_{e}} .
$$

The weak interaction is the only interaction in which both the electric charge of the fermions involved and their flavor quantum numbers may change. The change of charge follows from the fact that the $W^{ \pm}$field quanta (the boson carriers of the weak interaction) carry electrical charges. Also, only left-handed quarks feel the weak force, right-handed ones do not. In this document, we always refer only to left-handed quarks, and therefore, drop the prefix "left-handed" from now on.

\subsection{Charged weak decays}

In the SM description of the weak interaction, the weak eigenstates of downtype quarks $\left(d^{\prime}, s^{\prime}\right.$, and $\left.b^{\prime}\right)$ are mixtures of the mass eigenstates $(d, s$, and $b)$. The 
up-type quarks $(u, c$, and $t)$ are unmixed. This allows an up-type quark to decay, via emission of a $W$, to any energetically allowed down-type quark.

For the first two quark generations, the transitions between quark types are described by a real transformation matrix [2]:

$$
\left(\begin{array}{l}
d^{\prime} \\
s^{\prime}
\end{array}\right)=\left(\begin{array}{cc}
\cos \theta_{c} & \sin \theta_{c} \\
-\sin \theta_{c} & \cos \theta_{c}
\end{array}\right)\left(\begin{array}{l}
d \\
s
\end{array}\right)
$$

In this description, the Cabibbo angle $\left(\theta_{c}\right)$ has been measured to be approximately 0.23 radians. Transitions with both $c \rightarrow s$ and $u \rightarrow d$ have matrix elements proportional to $\cos ^{2} \theta_{c}$. These transitions are called "Cabibbo favored". The transitions with either $c \rightarrow d$ or $s \rightarrow u$ have matrix elements proportional to $\sin \theta_{c}$ and decay rates proportional to $\sin ^{2} \theta_{c}$. Transitions with both $c \rightarrow d$ and $s \rightarrow u$ have matrix elements proportional to $\sin ^{2} \theta_{c}$ and decay rates proportional to $\sin ^{4} \theta_{c}$. Decay rates proportional to $\sin ^{2} \theta_{c}$ are called singly Cabibbo-suppressed and those proportional to $\sin ^{4} \theta_{c}$ are called doubly Cabibbo-suppressed. The Feynman diagrams of the Cabibbo-favored $D^{0} \rightarrow K^{-} \pi^{+}$, the singly Cabibbo-suppressed $D^{0} \rightarrow K^{-} K^{+}$, and the doubly Cabibbo-suppressed $D^{0} \rightarrow K^{+} \pi^{-}$decays are shown in Figure 1.1.

For three generations of quarks, the above matrix is generalized, and is replaced with a complex matrix, called the CKM matrix [2], which describes the "mixing" between different quark generations and gives weak eigenstates.

$$
\left(\begin{array}{c}
d^{\prime} \\
s^{\prime} \\
b^{\prime}
\end{array}\right)=\left(\begin{array}{lll}
V_{u d} & V_{u s} & V_{u b} \\
V_{c d} & V_{c s} & V_{c b} \\
V_{t d} & V_{t s} & V_{t b}
\end{array}\right)\left(\begin{array}{c}
d \\
s \\
b
\end{array}\right)
$$

In this formalism, the transition rates between different quark generations are described by the values of the elements of $V$. As an example, the Cabibbo-suppressed $c \rightarrow d$ transition rate is proportional to $\left|V_{c d}\right|^{2}$. In the CKM matrix, the diagonal elements are near unity while the off-diagonal elements are small. Enforcing the unitarity of this matrix provides additional constraints on the values of its elements.

For the Cabibbo suppressed decay $D^{0} \rightarrow K^{-} K^{+}$shown in Figure 1.1, the transition probability is proportional to $\left|V_{c s}\right|^{2}\left|V_{u s}\right|^{2}$; the factor of $V_{c s}$ in the matrix element for the vertex where the $c$ quark turns into an $s$ quark and the factor $V_{u s}$ comes from the $W$ decay vertex.

\subsection{Weak decays of charmed mesons}

Charm quark decays to $s$ or $d$ via emission of a $W$ under the weak interaction. The $W$ may decay leptonically or hadronically to form a final state. Figure 1.2 shows the hadronic and leptonic decays of $W$ produced with $c \rightarrow s(d)$ transitions. The quarks produced this way form a hadronic final state under the strong interaction. The decays $D^{0} \rightarrow \pi^{-} \pi^{+} \pi^{0}$ and $D^{0} \rightarrow K^{-} K^{+} \pi^{0}$ are singly Cabibbo-suppressed while the $D^{0} \rightarrow K^{-} \pi^{+} \pi^{0}$ is a Cabibbo-favored decay. Figure 1.3 shows all the possible tree-level Feynman diagrams for the decay $D^{0} \rightarrow \pi^{-} \pi^{+} \pi^{0}$. Figure 1.4 shows the same for the decay $D^{0} \rightarrow K^{-} K^{+} \pi^{0}$. 


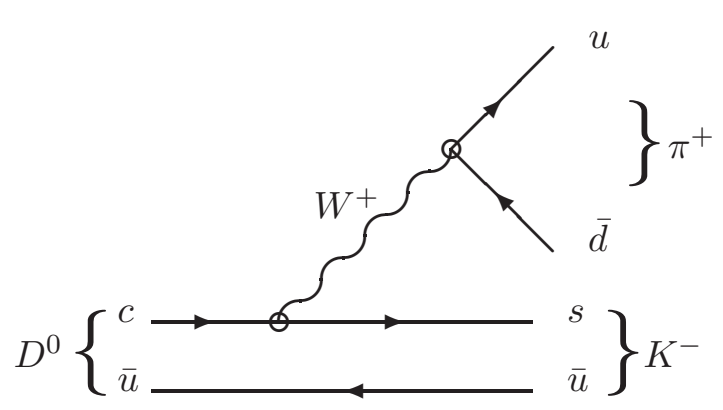

(a) Cabibbo-favored

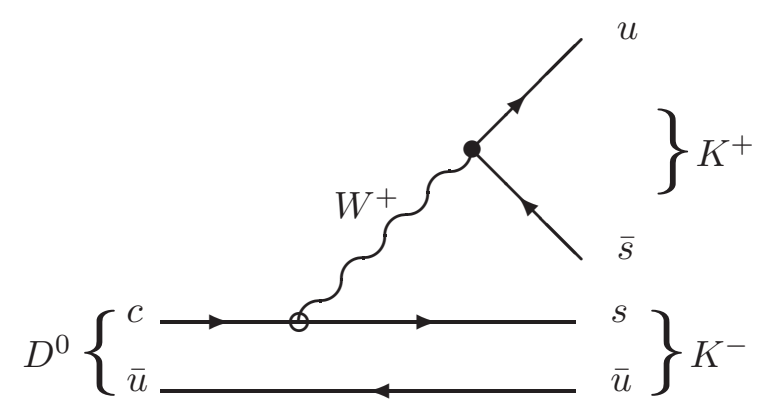

(b) Cabibbo-suppressed

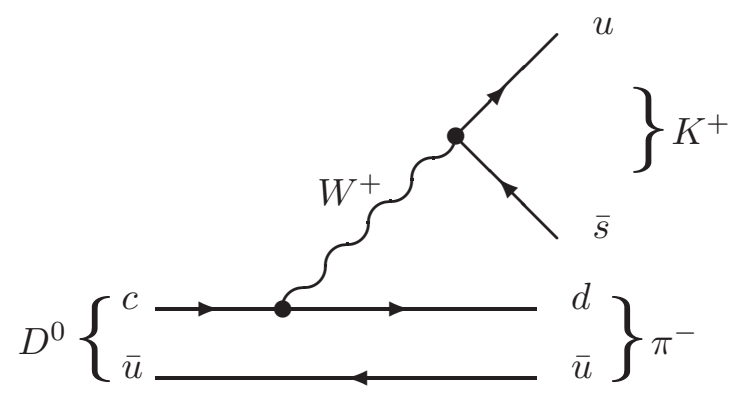

(c) Doubly Cabibbo-suppressed

Figure 1.1: Examples of (a) Cabibbo-favored, (b) singly Cabibbo-suppressed, and (c) doubly Cabibbo-suppressed decays. The weak vertices shown with open circles are Cabibbo-favored while those shown with filled circles are Cabibbo-suppressed. 


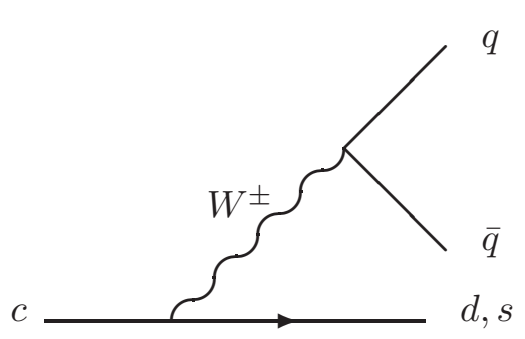

(a) Hadronic decay

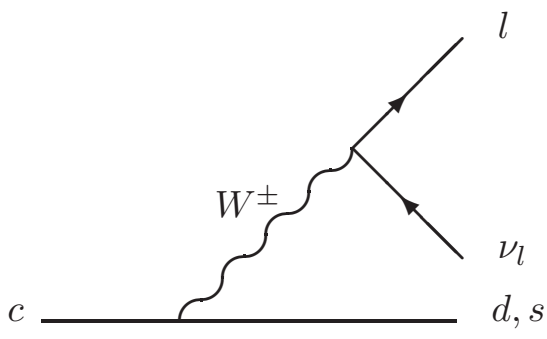

(b) Semi-leptonic decay

Figure 1.2: Hadronic and semi-leptonic decay of the charm quark.

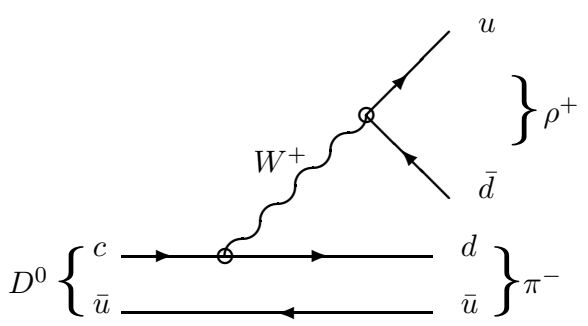

(I) Vector meson at $\mathrm{W}$ vertex

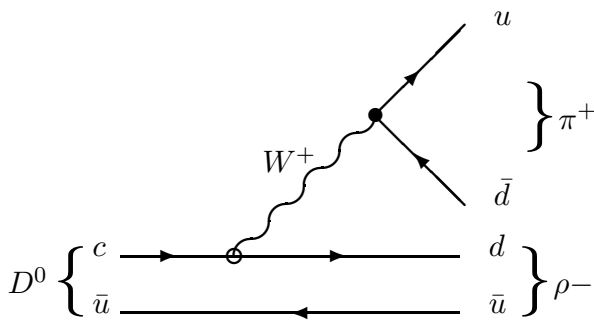

(II) Scalar at $\mathrm{W}$ vertex

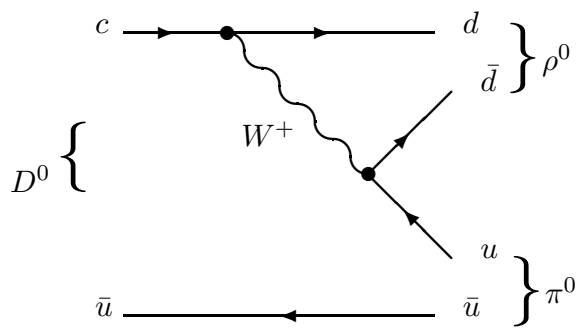

(III) Color-suppressed

Figure 1.3: Three types of tree-level Feynman diagrams for the decay $D^{0} \rightarrow \pi^{-} \pi^{+} \pi^{0}$.

\subsection{Introduction to $C P$-violating CKM phase $\gamma$}

The angle $\gamma$ of the unitarity triangle is the phase of the CKM matrix defined as $\gamma \equiv \arg \left[-V_{u d} V_{u b}^{*} / V_{c d} V_{c b}^{*}\right]$, which corresponds to the phase of the element $V_{u b}^{*}$, i.e. 


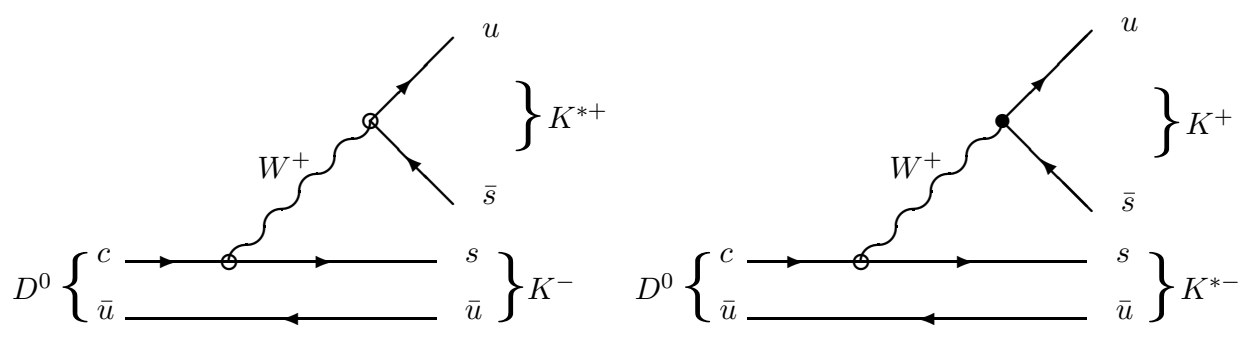

(I) Vector meson at $\mathrm{W}$ vertex

(II) Scalar at $\mathrm{W}$ vertex

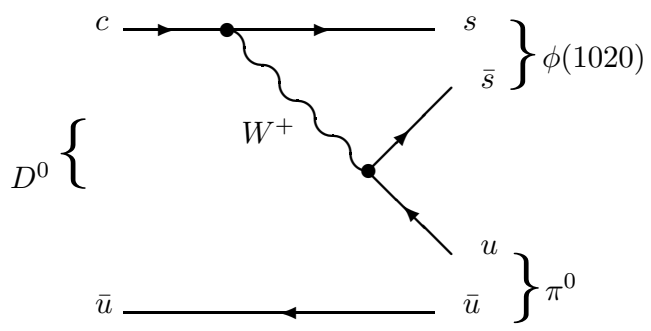

(III) Color-suppressed

Figure 1.4: Three types of tree-level Feynman diagrams for the decay $D^{0} \rightarrow$ $K^{-} K^{+} \pi^{0}$.

$V_{u b}=\left|V_{u b}\right| e^{-i \gamma}$, in the Wolfenstein parameterization [3]. Various methods have been proposed to extract $\gamma$ using $B^{\mp} \rightarrow \tilde{D}^{0} K^{\mp}$ decays, all exploiting the interference between the color allowed $B^{-} \rightarrow D^{0} K^{-}\left(b \rightarrow c \bar{u} s \propto V_{c b}\right)$ and the color suppressed $B^{-} \rightarrow \overline{D^{0}} K^{-}\left(b \rightarrow u \bar{c} s \propto V_{u b}\right)$ transitions ${ }^{1}$, when the $D^{0}$ and $\bar{D}^{0}$ are reconstructed in a common final state $[4,5,6]$. The symbol $\tilde{D}^{0}$ indicates either a $D^{0}$ or a $\bar{D}^{0}$ meson. The extraction of $\gamma$ with these decays is theoretically clean because the main contributions to the amplitudes come from tree-level diagrams (see Fig. 1.5).

\subsection{Measurement of phase $\gamma$}

The most effective method to measure the $C P$-violating phase $\gamma$ of the quark-mixing matrix is to exploit the interference between $b \rightarrow u \bar{c} s$ and $b \rightarrow c \bar{u} s$ amplitudes in the decay $B^{-} \rightarrow D K^{-}$where the neutral $D$ meson decays to a multi-body final state $\left(D \rightarrow \pi^{+} \pi^{-} \pi^{0}\right.$ in our case $\left.{ }^{2}\right)$. The sensitivity to $\gamma$ arises from the interference of the Cabibbo-allowed $B^{-} \rightarrow D^{0} K^{-}$decay and the doubly Cabibbo-suppressed decay

\footnotetext{
${ }^{1}$ Reference to the charge-conjugate state is implied here.

${ }^{2} D$ denotes either $D^{0}$ or $\bar{D}^{0}$ here.
} 

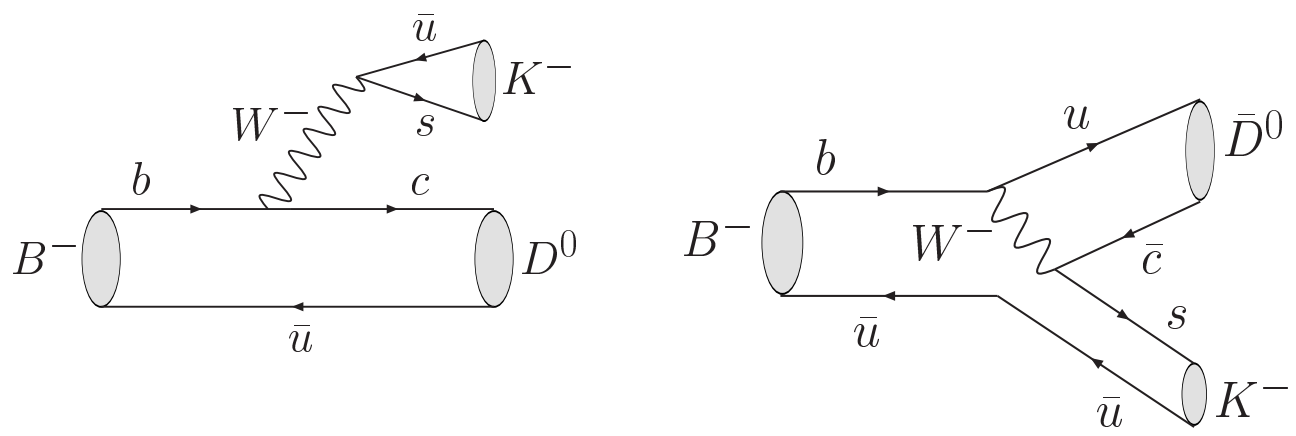

Figure 1.5: Diagrams contributing to $B^{-} \rightarrow \tilde{D}^{0} K^{-}$decay. The left diagram proceeds via $b \rightarrow c \bar{u} s$ transition, while the right diagram proceeds via $b \rightarrow u \bar{c} s$ transition and is color suppressed.

$B^{-} \rightarrow \bar{D}^{0} K^{-}$with $D \rightarrow \pi^{+} \pi^{-} \pi^{0}$ in both cases. We define the amplitudes

$$
\begin{aligned}
A\left(B^{-} \rightarrow D^{0} K^{-}\right) & \equiv A_{B} \\
A\left(B^{-} \rightarrow \bar{D}^{0} K^{-}\right) & \equiv A_{B} r_{B} e^{i\left(\delta_{B}-\gamma\right)}
\end{aligned}
$$

where we define all phases and amplitudes relative to the $B^{-} \rightarrow D^{0} K^{-}$decay. Therefore $\delta_{B}$ is the difference in the strong phase (sometimes also referred to as " $C P$ even phase") between the two decays and $\gamma$ is the weak phase. The amplitude for the $C P$ conjugate $B^{+}$decay is obtained by changing the sign of the weak phase $\gamma \rightarrow-\gamma$. The amplitude, $f$, of the 3 -body $D$-decay is parametrized in terms of its Dalitz plot variables ${ }^{3}$ :

$$
\begin{aligned}
A\left(D^{0} \rightarrow \pi^{-} \pi^{+} \pi^{0}\right) & \equiv f_{D^{0}}\left(s_{+0}, s_{-0}\right) \\
A\left(\bar{D}^{0} \rightarrow \pi^{+} \pi^{-} \pi^{0}\right) & \equiv f_{D^{0}}\left(s_{-0}, s_{+0}\right)
\end{aligned}
$$

where we have used the $C P$ symmetry of the strong interactions and the fact that the final state is a spin zero state, $p_{i}$ is the 4-momentum of the pion and $s_{i j}=\left(p_{i}+p_{j}\right)^{2}$ is the invariant mass squared of the pair of pions whose charges are given by $i$ and $j$. Note that the choice of the Dalitz variables leads to a symmetric Dalitz plot boundary. With the above definitions, the amplitude of the cascade decay is

$$
\begin{aligned}
& A_{B^{-} \rightarrow D_{\pi^{+} \pi^{-} \pi^{0}} K^{-}}\left(s_{+0}, s_{-0}\right)=A_{B}\left(f_{D^{0}}\left(s_{+0}, s_{-0}\right)+z_{-} f_{D^{0}}\left(s_{-0}, s_{+0}\right)\right), \\
& A_{B^{+} \rightarrow D_{\pi^{+} \pi^{-} \pi^{0}} K^{+}}\left(s_{+0}, s_{-0}\right)=A_{B}\left(f_{D^{0}}\left(s_{-0}, s_{+0}\right)+z_{+} f_{D^{0}}\left(s_{+0}, s_{-0}\right)\right),
\end{aligned}
$$

where

$$
z_{ \pm} \equiv r_{B} e^{i\left(\delta_{B} \pm \gamma\right)}
$$

With a specific model for the Dalitz amplitude $f_{D^{0}}$, determined separately from a $D^{0}$ sample from $D^{*+} \rightarrow D^{0} \pi^{+}$decays, one can extract the $C P$ parameters $r_{B}, \delta_{B}$, and $\gamma$ (or the Cartesian coordinates $x^{ \pm}, y^{ \pm}$, where $z_{ \pm}=x_{ \pm}+i y_{ \pm}$) using a simultaneous fit

\footnotetext{
${ }^{3}$ We neglect any direct $C P$ violation in the neutral $D$-decay here.
} 
to $B^{-} \rightarrow D_{\pi^{+} \pi^{-} \pi^{0}} K^{-}$and $B^{+} \rightarrow D_{\pi^{+} \pi^{-} \pi^{0}} K^{+}$events. We describe the measurement of the amplitudes of the decay $D^{0} \rightarrow \pi^{-} \pi^{+} \pi^{0}$ in Chapter 5 . In Chapter 4 we describe the amplitudes of the decay $D^{0} \rightarrow K^{-} K^{+} \pi^{0}$.

We note that not only the functional form of the $f_{D^{0}}$ but also the absolute magnitudes of the squares of the amplitudes in Eq. (1.6) can be used to obtain information about the $C P$ parameters from the decay $B^{-} \rightarrow D_{\pi^{+} \pi^{-} \pi^{0}} K^{-}$. We describe this in detail in Chapter 6.

\subsection{Search for $C P$ violation in charm decays}

$C P$ violation [7] occurs when the decay rate of a particle (e.g., $\left.D^{0}\right)$ differs from the decay rate of its $C P$-conjugate anti-particle (e.g., $\bar{D}^{0}$ ). This phenomenon requires at least two intefering complex quantum mechanical amplitudes with different strong and weak phases. The strong phase of each amplitude always respects $C P$ symmetry while the weak phase changes sign under $C P$. Decay rates are proportional to the square of the magnitude of the complex sum of the quantum mechanical amplitudes, therefore rates for particle and anti-particle can differ. In the Standard Model, the relative weak phase is typically between "tree level" and SM "penguin" amplitudes. The penguin amplitude in charm decays is, however, too small to provide significant $C P$ violation. Extensions of the SM, which introduce new forces, introduce additional amplitudes with relative weak phases that can contribute to $C P$ violation. So, at current experimental sensitivities, a search for $C P$ violation in charm decays [8] is a way to look for physics beyond the SM. Any observation of direct $C P$ violation $[9$, $10,11]$ will provide a "smoking gun" signal of new physics.

\section{8 $C P$ asymmetry in Cabibbo-suppressed $D^{0}$ decays}

Singly Cabibbo-suppressed (SCS) neutral $D$ decays have the amplitudes of the form $c \rightarrow u \bar{s} s$ and $c \rightarrow u \bar{d} d$ (and the respective $C P$-conjugates). These amplitudes lead to final states which are common to both $D^{0}$ and $\bar{D}^{0}$. These states can be $C P$ eigenstates (e.g., $\phi(1020) \pi^{0}, \rho^{0} \phi^{0}, f_{0}(980) \pi^{0}$ ), or flavor states (e.g., $\rho^{ \pm} \pi^{\mp}, K^{* \pm} K^{\mp}$ ).

The time-integrated $C P$ asymmetry, $a_{C P}$, for a final state $f$ can be written as:

$$
a_{C P} \equiv \frac{\Gamma\left(D^{0} \rightarrow f\right)-\Gamma\left(\bar{D}^{0} \rightarrow f\right)}{\Gamma\left(D^{0} \rightarrow f\right)+\Gamma\left(\bar{D}^{0} \rightarrow f\right)},
$$

where $\Gamma$ denotes the decay rate.

Time-integrated $C P$ asymmetries in $D^{0}$ decays can have three components: direct $C P$ violation in decays to specific states, indirect $C P$ violation in $D^{0}-\bar{D}^{0}$ mixing, and indirect $C P$ violation in interference of decays with and without mixing. Indirect $C P$ violation should be universal, but direct $C P$ violation can be non-universal depending on the specifics of the new physics [9]. We search for time-integrated $C P$ violation in the three-body SCS decays $D^{0} \rightarrow \pi^{-} \pi^{+} \pi^{0}, K^{-} K^{+} \pi^{0}$ by comparing features of $D^{0}$ and $\bar{D}^{0}$. We will describe this in detail in Chapter 7 . 


\section{Chapter 2}

\section{BABAR Detector and Event Reconstruction}

\subsection{The BABAR experiment}

The primary physics goal of the BABAR [12] experiment is the systematic study of $C P$ violating asymmetries in the decay of neutral $B$ mesons to $C P$ eigenstates. Secondary goals are precision measurements of decays of beauty and charm mesons and searches for rare processes. The PEP-II $B$ Factory is an asymmetric $e^{+} e^{-}$collider designed to operate at a luminosity of $3 \times 10^{33} \mathrm{~cm}^{-2} \mathrm{~s}^{-1}$ and above, at a center-of-mass energy of $10.58 \mathrm{GeV}$, the mass of the $\Upsilon(4 S)$ resonance. This resonance decays exclusively to $B^{0} \bar{B}^{0}$ and $B^{+} B^{-}$pairs and thus provides an ideal laboratory for the study of $B$ mesons. Also, continuum production of $b \bar{b}, c \bar{c}, s \bar{s}, d \bar{d}$, and $u \bar{u}$ processes at the enegies of around $10.58 \mathrm{GeV}$ enables the study of other physics topics. $B^{0}$ mixing had been discovered in 1987 [13] and it was soon proposed to use mixing to measure $C P$ violation using the time-difference between neutral $B$ decays [14]. An asymmetric collider makes this possible by allowing the $B$ 's from the $\Upsilon(4 S)$ decay to move in the laboratory reference frame. The two separate energy rings (one each for $e^{-}$ and $e^{+}$) also allow high beam currents and high luminosities [15]. While most of the $B A B A R$ data are recorded at the peak of the $\Upsilon(4 S)$ resonance, about $10 \%$ are taken at a center-of-mass energy $40 \mathrm{MeV}$ lower. In PEP-II, the electron beam of 9.0 GeV collides head-on with the positron beam of $3.1 \mathrm{GeV}$ resulting in a Lorentz boost to the $\Upsilon(4 S)$ resonance of $\beta \gamma=0.56$. The crucial test of $C P$ invariance is a comparison of the time-dependent decay rates for $B^{0}$ and $\bar{B}^{0}$ to a self-conjugate state. This requires events in which one $B$ meson decays to a $C P$ eigenstate that is fully reconstructed and the other $B$ meson is tagged as a $B^{0}$ or a $\bar{B}^{0}$ by its decay products: a charged lepton, a charged kaon, or other flavor sensitive features such as a low momentum charged pion from a $D^{*}$ decay.

The peak cross section at the $\Upsilon(4 S)$ is about $1.1 \mathrm{nb}$ for $b \bar{b}$ events and about $1.8 \mathrm{nb}$ for $c \bar{c}$ events. A brief overview of the principal components of the BABAR detector is given in the next section. 


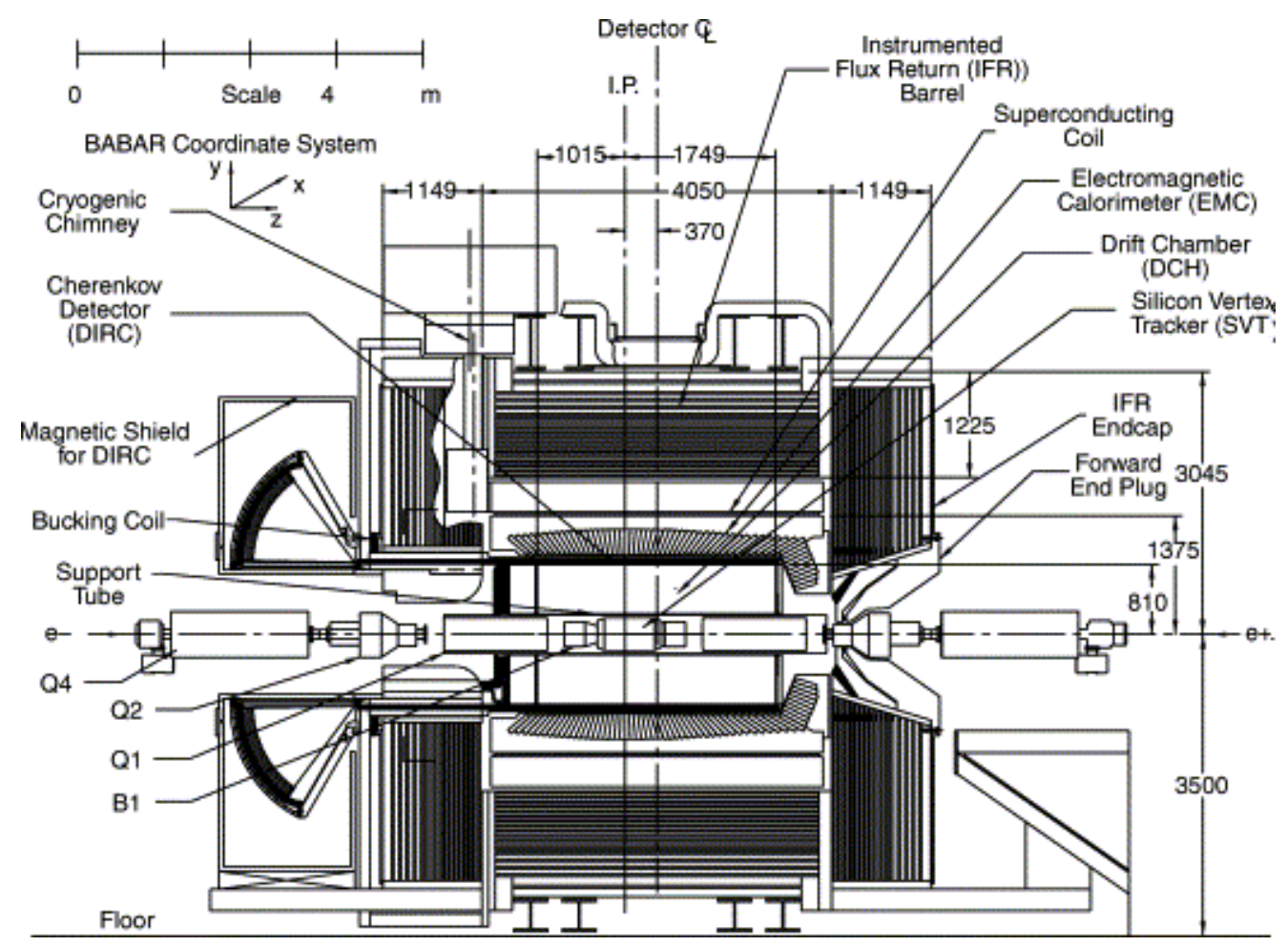

Figure 2.1: BABAR detector longitudinal section (source Ref. [12]).

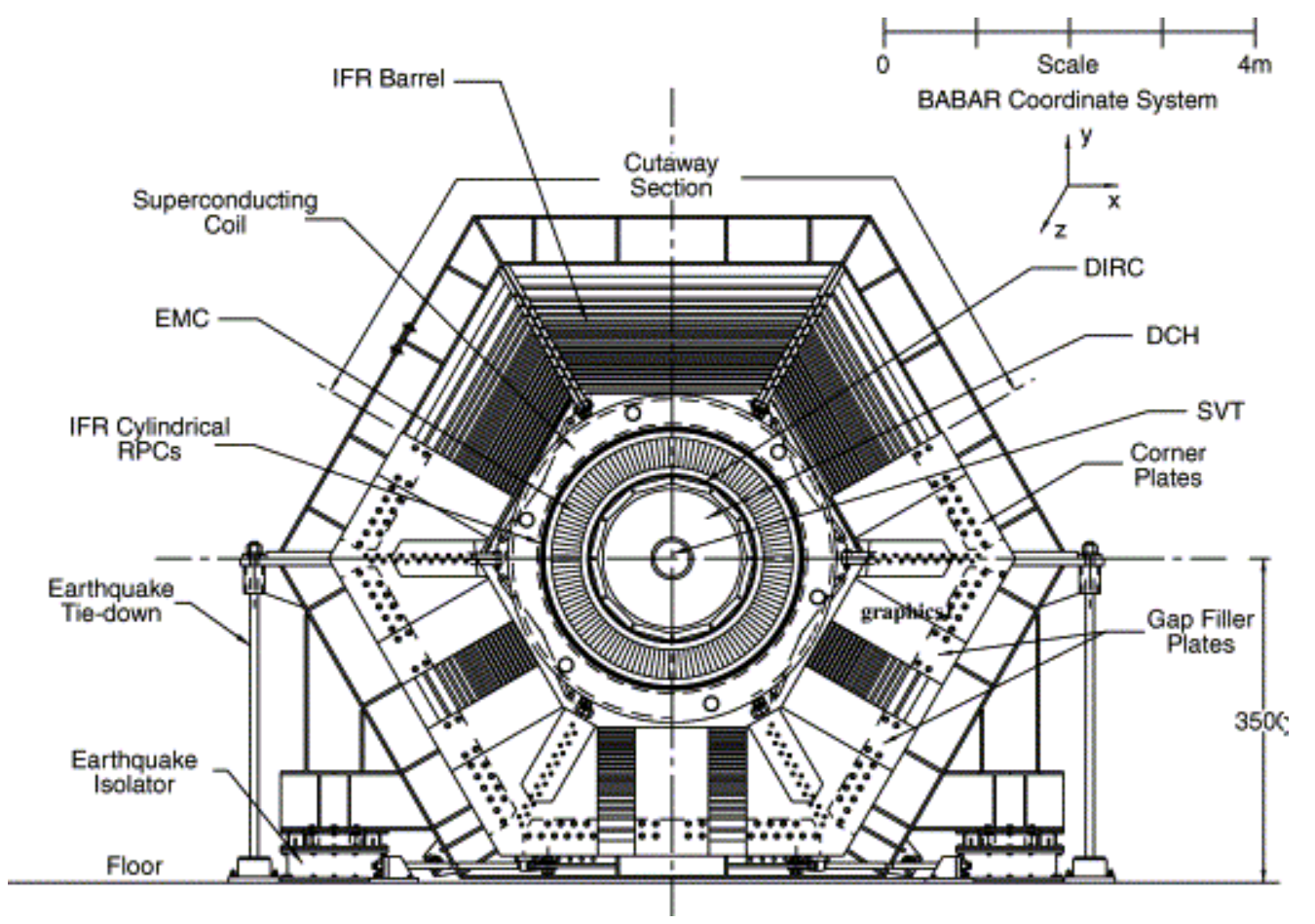

Figure 2.2: BABAR detector end view (source Ref. [12]). 


\subsection{Detector overview}

Figure 2.1 shows a longitudinal section through the detector center, and Figure 2.2 shows an end view with the principal dimensions. The detector surrounds the PEPII interaction region. The inner detector consists of a silicon vertex tracker, a drift chamber, a ring-imaging Cherenkov detector, and a CsI calorimeter. These detector systems are surrounded by a superconducting solenoid that is designed for a field of 1.5 T. The steel flux return is instrumented for muon and neutral hadron detection. The polar angle coverage extends to $350 \mathrm{mrad}$ in the forward direction and $400 \mathrm{mrad}$ in the backward direction, defined relative to the high energy beam. As indicated in the two drawings, the right handed coordinate system is anchored on the main tracking system, the drift chamber, with the $z$-axis coinciding with its principal axis. The positive $y$-axis points upward and the positive $x$-axis points away from the center of the PEP-II storage rings.

\subsubsection{Detector components}

The charged particle tracking system is made of two components, the silicon detector and the drift chamber.

The silicon detector is designed to measure angles and positions of charged particles just outside the beam pipe. It is composed of five layers of double-sided silicon strips. The inner three layers primarily provide position and angle information for the measurement of the vertex position. The outer two layers are at much larger radii, providing the coordinate and angle measurements needed for linking the tracks reconstructed in the silicon detector and in the drift chamber.

The principal purpose of the drift chamber is the momentum measurement for charged particles. It also supplies information for the charged particle trigger and a measurement of $\mathrm{d} E / \mathrm{d} x$ for particle identification. The drift chamber is of compact design, with 40 layers of small, approximately hexagonal cells. Longitudinal information is derived from wires placed at small angles to the principal axis. The helium/isobutane gas mixture reduces the scattering and improves the measurement of low momentum tracks [16].

The detector [17] of internally reflected Cherenkov light, also referred to as "particle identification system", is a novel device providing separation of pions and kaons from about $500 \mathrm{MeV} / c$ to the kinematic limit of $4.5 \mathrm{GeV} / c$. Cherenkov light is produced in bars of synthetic fused silica and transported by total internal reflection, preserving the angle of emission, to an array of photomultiplier tubes. Images of the Cherenkov rings are reconstructed from the position and time of arrival of the signals in the photomultiplier tubes.

The electromagnetic calorimeter is designed to detect electromagnetic showers with excellent energy and angular resolution over the energy range from $20 \mathrm{MeV}$ to $4 \mathrm{GeV}$. This coverage allows the detection of low energy $\pi^{0} \mathrm{~s}$ and $\eta^{0} \mathrm{~s}$ and higher energy photons and electrons from electromagnetic, weak, and radiative processes. The calorimeter is a finely segmented array of thallium-doped cesium iodide (CsI( $\mathrm{Tl})$ ) crystals. The individual crystals are read out by pairs of silicon PIN diodes.

The instrumented flux return is designed to identify muons. The electronics, trigger, data acquisition, and online computing represent a collection of tightly cou- 
pled hardware and software systems. These systems were designed to maximize the physics data acceptance, maintainability, and reliability while managing complexity, and minimizing deadtime.

\subsubsection{Trigger}

The trigger system operates as a sequence of two independent stages, the second conditional upon the first. The Level-1 (first stage) trigger is responsible for interpreting incoming detector signals, recognizing and removing beam-induced background to a level acceptable for the Level-3 (second stage) software trigger which runs on a farm of commercial processors. Level-1 consists of pipelined hardware processors designed to provide an output trigger rate of $\lesssim 2 \mathrm{kHz}$. The Level- 1 trigger selection is based on data from the drift chamber and the calorimeter. The maximum Level-1 response latency for a given collision is $12 \mu \mathrm{s}$. Based on both the complete event and Level-1 trigger information, the Level-3 software algorithms select events of interest which are then stored for processing. The Level- 3 output rate is typically around $160 \mathrm{~Hz}$.

\subsubsection{Beam parameters}

PEP-II is an $e^{+} e^{-}$storage ring system designed to operate at a center-of-mass (c.m.) energy of $10.58 \mathrm{GeV}$, corresponding to the mass of the $\Upsilon(4 S)$ resonance. The parameters of these energy asymmetric storage rings are presented in Table 2.1.

In the interaction region, closely-spaced bunches of electrons and positrons collide head-on and are separated magnetically in the horizontal plane by a pair of dipole magnets, followed by a series of offset quadrupoles. The beam parameters most critical for BABAR performance are the luminosity, the energies of the two beams, and the position, angles, and size of the luminous region. BABAR measures the absolute luminosity from QED processes, primarily $e^{+} e^{-}$, and $\mu^{+} \mu^{-}$pairs. The rms energy spreads of the LER and HER beams are $2.3 \mathrm{MeV}$ and $5.5 \mathrm{MeV}$, respectively. To ensure that data are recorded close to the peak of the $\Upsilon(4 S)$ resonance, the observed ratio of $B \bar{B}$ enriched hadronic events to lepton pair production is monitored online. Near the peak of the resonance, a $2.5 \%$ change in the $B \bar{B}$ production rate corresponds to a $2 \mathrm{MeV}$ change in the c.m. energy. The direction of the beams relative to BABAR is measured iteratively run-by-run using $e^{+} e^{-} \rightarrow e^{+} e^{-}$and $e^{+} e^{-} \rightarrow \mu^{+} \mu^{-}$events. The resultant uncertainty in the direction of the boost from the laboratory to the c.m. frame, $\vec{\beta}$, is about $1 \mathrm{mrad}$.

\subsubsection{Beam background sources}

The primary sources of accelerator backgrounds are: the beam-gas bremsstrahlung and Coulomb scattering between the beam particles and the residual gas in either ring; and electromagnetic showers generated by beam-beam collisions. Since the magnet bends the particles from the two beams in opposite directions, most BABAR detector systems exhibit occupancy peaks in the horizontal plane, i.e., the LER background near $\phi=0^{\circ}$ and HER background near $\phi=180^{\circ}$. 
Table 2.1: PEP-II beam parameter values for a typical colliding beam operation. HER and LER refer to the high energy $e^{-}$and low energy $e^{+}$ring, respectively. $\sigma_{L x}, \sigma_{L y}$, and $\sigma_{L z}$ refer to the horizontal, vertical, and longitudinal rms size of the luminous region.

\begin{tabular}{lc}
\hline Parameters & Values \\
\hline Energy HER/LER $(\mathrm{GeV})$ & $9.0 / 3.1$ \\
Current HER/LER $(\mathrm{A})$ & $1.8 / 3.0$ \\
$\sigma_{L x}(\mu \mathrm{m})$ & 120 \\
$\sigma_{L y}(\mu \mathrm{m})$ & 5.6 \\
$\sigma_{L z}(\mathrm{~mm})$ & 9 \\
Luminosity $\left(10^{33} \mathrm{~cm}^{-2} \mathrm{~s}^{-1}\right)$ & $8-12$ \\
\hline
\end{tabular}

\subsection{Data sample}

The analyses described in this document are done selecting $D^{*+} \rightarrow D^{0} \pi_{s}^{+}, D^{0} \rightarrow$ $h^{-} h^{+} \pi^{0}$ and charge-conjugate events, where $h$ refers to $\pi$ or $K$. The analyses use either the BABAR Runs 1-4 (integrated luminosity, $L=\frac{\# \text { events }}{\text { cross-section }}$, being roughly $\left.232 \mathrm{fb}^{-1}\right)$ or the Runs $1-5\left(385 \mathrm{fb}^{-1}\right)$ data sample. This includes on-resonance and off-resonance data. The size of the data-sample used in a particular analysis is mentioned in the respective chapters. For optimization of analysis variables, we use similated events: $e^{+} e^{-} \rightarrow b \bar{b}\left(2045 \mathrm{fb}^{-1}\right), c \bar{c}\left(327.5 \mathrm{fb}^{-1}\right)$ and also $u \bar{u}, d \bar{d}, s \bar{s}$ $\left(322.5 \mathrm{fb}^{-1}\right)$. Special signal Monte Carlo samples of $D^{0} \rightarrow K^{-} \pi^{+} \pi^{0}$ (4.7 million), $D^{0} \rightarrow \pi^{-} \pi^{+} \pi^{0}$ (4.7 million), and $D^{0} \rightarrow K^{-} K^{+} \pi^{0}$ ( 1.4 million ) signal modes ( $D^{0}$ and $\bar{D}^{0}$ combined, generated uniformly in phase space) are also used to study detector acceptance and signal-reconstruction efficiency.

\subsection{Charged particle track reconstruction}

Charged particle tracks are reconstructed by using information from the drift chamber and the silicon detector. They are found by fitting the expected helices formed by charged particles in the magnetic field to the sequences of hits. The tracks are parameterized by five parameters $\left(d_{0}, z_{0}, \phi_{0}, \omega, \tan \lambda\right)$. These parameters are measured at the point of closest approach to the z-axis. $d_{0}$ is the distance of this point from the origin of the coordinate system in the $\mathrm{x}-\mathrm{y}$ plane and $z_{0}$ is that distance along the z-axis. $\phi_{0}$ is the azimuthal angle, $\lambda$ the dip angle relative to the transverse plane, and $\omega=1 / p_{T}$ is the curvature of the track, $p_{T}$ being the transverse momentum. The signs of $d_{0}$ and $\omega$ depend on the charge of the particle.

The particles actually observed in the detector are pions, electrons, muons, kaons, protons and photons. All other particles that have decayed in flight are reconstructed from these long-lived particles. The criteria used to select charged particle tracks used in the present analyses are summarized in Table 2.2 . 


\begin{tabular}{ll}
\hline Variable & Selection \\
\hline \hline$p_{T}$ & $>0.1 \mathrm{GeV} / \mathrm{c}$ \\
$\#$ drift chamber hits & $\geq 12$ \\
$\left|x_{0}\right|$ & $<1.5 \mathrm{~cm}$ \\
$\left|z_{0}\right|$ & $<10.0 \mathrm{~cm}$ \\
\hline
\end{tabular}

Table 2.2: The basic track selection criteria used for charged particles. $\left|x_{0}\right|$ is the absolute value of the closet distance between the track and the beam spot in the $x-y$ plane.

\section{$2.5 \pi^{0}$ reconstruction}

The $\pi^{0}$ candidates are formed by combining a pair of photon candidates. The invariant mass of the photon pair is required to be within $0.115<M(\gamma, \gamma)<0.150$ $\mathrm{GeV} / c^{2}$ and each photon has energy at least $100 \mathrm{MeV}$. Selected $\gamma$ candidates are kinematically fitted so that the $\gamma \gamma$ invariant mass is equal to the nominal $\pi^{0}$ mass. Also, the $\pi^{0}$ energy is required to be greater than $350 \mathrm{MeV}$. Both photons in the pair are also required to have at least one hit in the calorimeter and $L A T<0.8$. Here $L A T$ is the lateral energy distribution variable and is defined as

$$
L A T=\frac{\sum_{i=3}^{N} E_{i} r_{i}^{2}}{\sum_{i=3}^{N} E_{i} r_{i}^{2}+E_{1} r_{0}^{2}+E_{2} r_{0}^{2}}, E_{1} \geq E_{2} \geq \ldots \ldots \geq E_{n}
$$

where $N$ is the number of crystals in a shower, $r_{0}$ is the average distance between two crystal front faces (about $5 \mathrm{~cm}$ ), $E_{i}$ is the energy deposited in the $i^{\text {th }}$ crystal, and $r_{i}$ is the distance between the $i^{t h}$ crystal and the shower center. The LAT variable can be used to discriminate between electromagnetic and hadronic showers. For electromagnetic showers, most of the energy is deposited in a small number of crystals, so that one expects a low value for the LAT parameter. Hadronic showers, however, generally result in a distribution of energy which is more even over a number of crystals, resulting in a value for the LAT parameter which is closer to one.

\subsection{Charged particle identification}

Charged particle candidates are identified by specific energy-loss $(d E / d x)$ measurements in the tracking detectors together with the number of Cherenkov photons reconstructed in the particle identification system. For each particle hypothesis a likelihood $(L)$ is calculated using the above variables. Particles are identified using different cuts on the relevant likelihood ratios. Figure 2.3 (2.4) shows the kaon (pion) identification efficiency and the pion (kaon) mis-identification rate. The values of likelihood ratios and other parameters are summarized in Table 2.3.

\subsection{Event reconstruction}

Events for this analysis are pre-selected from a pool of events classified as "useful physics events" by the online prompt reconstruction algorithm. This includes events 

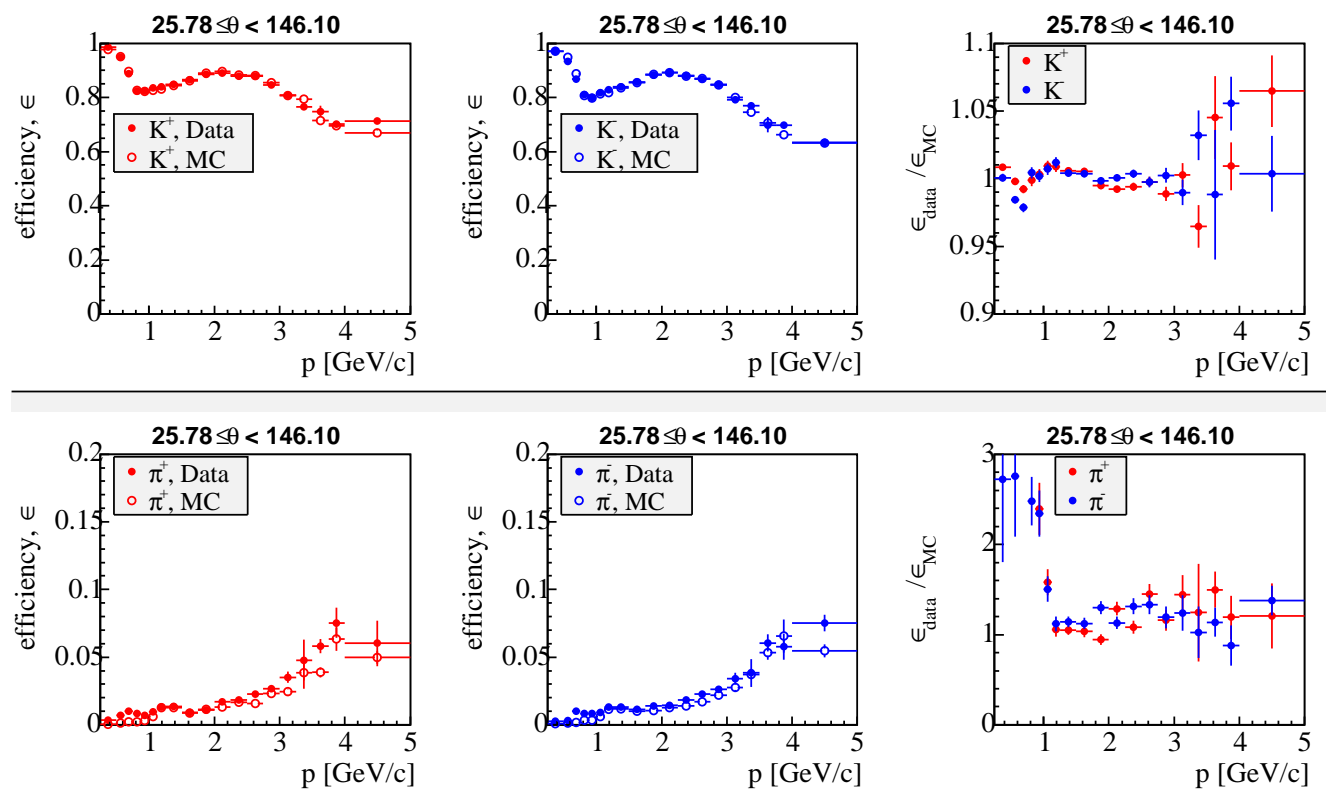

Figure 2.3: Kaon identification efficiency (top) and pion mis-identification rate (bottom) as a function of track momentum for positive (left) and negative (middle) tracks. The rightmost plots show ratios of efficiencies in data and simulation.
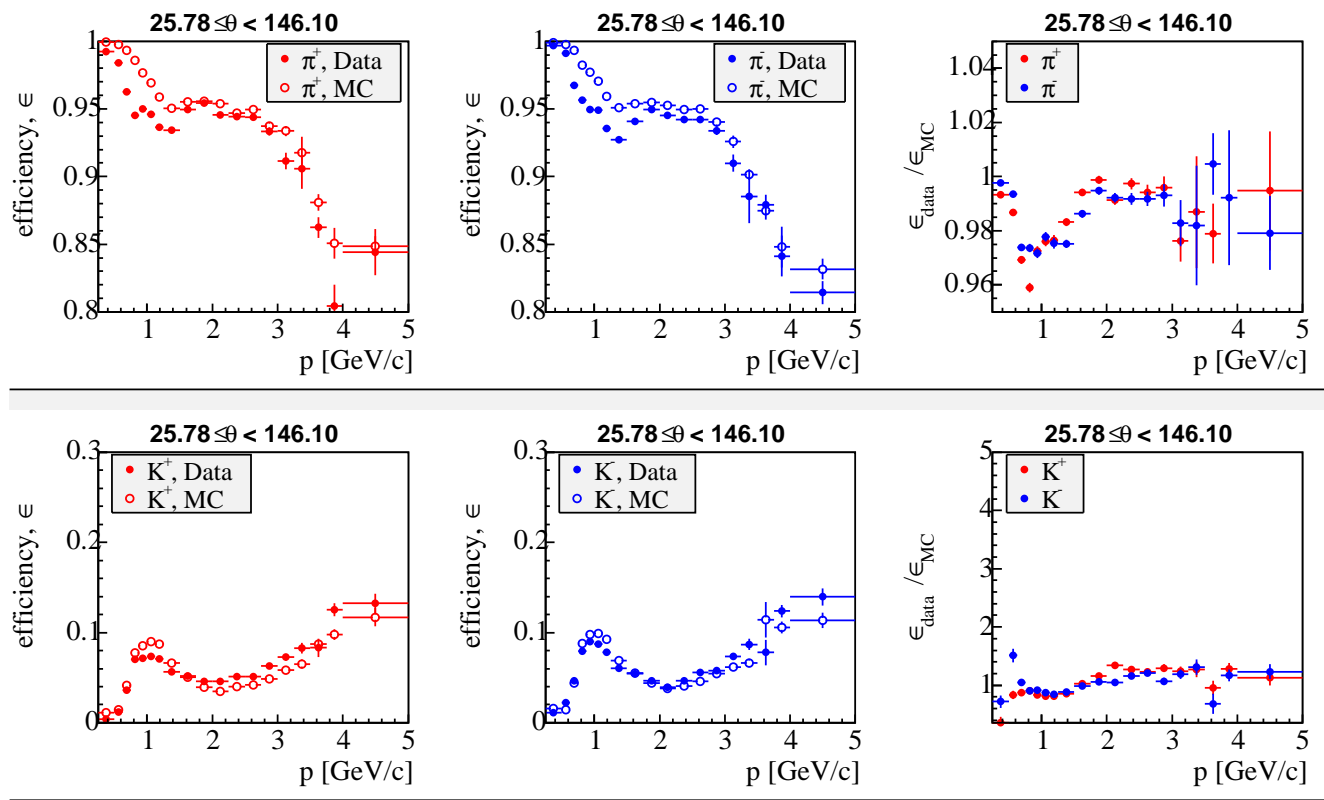

Figure 2.4: Pion identification efficiency (top) and kaon mis-identification rate (bottom) as a function of track momentum for negative (left) and positive (middle) tracks. The rightmost plots show ratios of efficiencies in data and simulation. 


\begin{tabular}{|l|l||l|}
\hline Parameter & For Kaons & For Pions \\
\hline$L_{\text {Kaon }} /\left(L_{\text {Kaon }}+L_{\text {Pion }}\right)$ & $>0.9$ & $<0.5$ \\
$L_{\text {Kaon }} /\left(L_{\text {Kaon }}+L_{\text {Proton }}\right)$ & $>0.2$ & - \\
$L_{\text {Proton }} /\left(L_{\text {Proton }}+L_{\text {Pion }}\right)$ & - & $<0.98$ \\
\hline
\end{tabular}

Table 2.3: Charged particle identification requirements. The tracks are also required to be inconsistent with electron hypothesis if their momentum is $>0.4 \mathrm{GeV} / c$.

assigned to any of the physics sub-streams by the background filter. The selected events are also required to belong to the class of hadronic events which contain at least three charged tracks. Using the criteria listed in Tables 2.2-2.3, the $D^{0} \rightarrow$ $h^{-} h^{+} \pi^{0}$ events are reconstructed going backward in the decay chain.

We select only those events where the $D^{0} \rightarrow h^{-} h^{+} \pi^{0}$ candidate comes from $D^{*}$ decays. Using two distinct pairs of oppositely charged tracks with $\pi^{ \pm}$and $K^{ \pm}$ mass hypotheses and a $\pi^{0}$, we form $D^{0}$ candidates by adding the four-momenta and minimizing the $\chi^{2}$ of the vertex. In order to suppress background from charged track combinatorics in $D^{0}$ reconstruction, we require both the charged tracks to have a minimum transverse momentum, $p_{T}, 100 \mathrm{MeV} / \mathrm{c}$. We also require them to have at least 20 drift chamber hits and at least 6 hits in the silicon tracker with a minimum of one hit in each of three inner layers. We also require the charged tracks to be within the fiducial volume of the Cherenkov detector acceptance range of lab-momentum and polar angle (because particle identification performances are known reliably in this range only). Similarly, in order to minimize background from fake soft pions in $D^{*}$ candidate reconstruction, we require the soft pion $p_{T}$ to be greater than 100 $\mathrm{MeV} / \mathrm{c}$, at least 20 drift chamber hits and at least 6 hits in silicon detector with a minimum of one hit each in the three inner layers.

To minimize background coming from $\pi^{0}$ mis-reconstruction, we require the $\pi^{0}$ candidate lab-energy to be greater than $350 \mathrm{MeV}$. We also constrain the $\pi^{0}$ candidate invariant mass to nominal $\pi^{0}$ mass in order to have a a good resolution in $D^{0}$ candidate mass.

At this stage we also use particle identification method, described earlier, to identify kaons and pions. At various stages of this process, we use appropriate selection criteria to reject background and to speed up the reconstruction process. We require the $D^{0}$ vertex-fit to have $\chi^{2}$ probability greater than $0.5 \%$, and the resultant invariant mass to lie between $1.7 \mathrm{GeV} / c^{2}$ and $2.0 \mathrm{GeV} / c^{2}$. This selection retains only events in the interesting mass window. The minimum center-of-mass momentum of the candidate is $2.77 \mathrm{GeV} / c$. This selection removes $B$ decay products and reduces high multiplicity events. The selected candidates after the above requirements are combined with the third charged track to make a $D^{*}$ candidate. The tracks are required to originate close to the beam spot.

To get a reasonably clean sample of $h^{-} h^{+} \pi^{0}$ events from data samples, we studied the $\Delta m\left(D^{*}-D^{0}\right.$ mass difference) distributions in data and Monte Carlo samples, in order to be able to apply optimal selection cuts on this variable. The mean value of $\Delta m$ is the same for data and simulation $\left(145.4 \mathrm{MeV} / c^{2}\right)$ and for all the three modes under study but the r.m.s. width is slightly different in data and 
simulation ( $\sim 0.32 \mathrm{MeV} / c^{2}$ in data and $\sim 0.25 \mathrm{MeV} / c^{2}$ in simulation), therefore any cut applied on $\Delta m$ will not have exactly the same effect on data and simulation. Since eventually we obtain the signal reconstruction efficiency using simulated signal events, it is important that we apply uniform selection cuts (as far as possible) to all the three modes under study so that any small discrepancy between data and simulation mostly cancels out in the ratio of branching ratio. Accordingly, we use a uniform $\Delta m$ selection, $|\Delta m-145.4|<0.6 \mathrm{MeV} / c^{2}$, for all the three decay modes under study, in both data and simulation. We study systematic uncertainty in our final result due to this selection, this will be described in detail in a later section.

If the $D^{*}$ candidate's vertex fit is good and the mass difference between the reconstructed $D^{*}$ and $D^{0}$ candidates is within $0.6 \mathrm{MeV} / c^{2}$ of the central value, then these events are the most probable $D^{0} \rightarrow h^{-} h^{+} \pi^{0}$ signal candidates.

The event selection criteria are summarized in Table 2.5 and Table 2.7 shows the reconstruction efficiency after successive cuts. Events which pass the above selections are investigated further for analysis.

\begin{tabular}{|c|c|c|}
\hline Decay & Mean $\left[\mathrm{MeV} / c^{2}\right]$ & $R M S\left[\mathrm{MeV} / c^{2}\right]$ \\
\hline \hline$K^{-} \pi^{+} \pi^{0}$ data & $145.42 \pm 0.01$ & $0.34 \pm 0.02$ \\
\hline$K^{-} \pi^{+} \pi^{0}$ simulation & $145.44 \pm 0.01$ & $0.26 \pm 0.04$ \\
\hline$\pi^{-} \pi^{+} \pi^{0}$ data & $145.42 \pm 0.01$ & $0.32 \pm 0.02$ \\
\hline$\pi^{-} \pi^{+} \pi^{0}$ simulation & $145.43 \pm 0.01$ & $0.26 \pm 0.01$ \\
\hline$K^{-} K^{+} \pi^{0}$ data & $145.42 \pm 0.01$ & $0.30 \pm 0.07$ \\
\hline$K^{-} K^{+} \pi^{0}$ simulation & $145.43 \pm 0.01$ & $0.24 \pm 0.01$ \\
\hline
\end{tabular}

Table 2.4: Mean and rms values of $\Delta m$ distribution for reconstructed $D^{0}$ candidates having mass within $1 \sigma$ of the central value.

\begin{tabular}{|c|c|}
\hline Variable & Cut \\
\hline Charged tracks and $\pi_{s} p_{T}$ & $\geq 100 \mathrm{MeV} / \mathrm{c}$ \\
Hits in the silicon strip detector & $\geq 6$ total, 2 in $\mathrm{r}-\phi$ plane, 1 in inner 3 layers \\
Hits in the drift chamber & $\geq 20$ \\
$|\Delta m-145.4|$ & $<0.6 \mathrm{MeV} / c^{2}$ \\
$P^{*}\left(D^{0} / \bar{D}^{0}\right)$ & $>2.77 \mathrm{GeV} / c$ \\
$E_{\gamma}$ & $>100 \mathrm{MeV}$ \\
$E_{\pi^{0}}$ & $>350 \mathrm{MeV}$ \\
$m_{\pi^{0}}$ & {$[115-160] \mathrm{MeV} / c^{2}$} \\
Kaon, pion identification & Yes \\
\hline
\end{tabular}

Table 2.5: Event selection criteria

\subsection{Dealing with multiple candidates}

When reconstructing the whole $D^{*}$ decay chain, it can sometimes happen that more than one combination satisfies the selection criteria in the same event. The rate 


\begin{tabular}{r|r|r|r}
\hline \hline Selection & \multicolumn{3}{|c}{ Efficiency after selection (in \%) } \\
\hline & $D^{0} \rightarrow \pi^{-} \pi^{+} \pi^{0}$ & $D^{0} \rightarrow K^{-} \pi^{+} \pi^{0}$ & $D^{0} \rightarrow K^{-} K^{+} \pi^{0}$ \\
\hline$P_{D^{0}}^{C M}>2.77 \mathrm{GeV} / c$ & 49.46 & 49.67 & 49.58 \\
Track quality requirements & 7.29 & 6.31 & 5.45 \\
$E_{\pi^{0}}>350 \mathrm{MeV}$ & 7.12 & 6.16 & 5.22 \\
$\Delta m$ selection & 6.23 & 5.51 & 4.74 \\
\hline
\end{tabular}

Table 2.6: Efficiency after successive selection requirements.

at which this occurs depends on the selection cuts applied but is the same for all the three $h^{-} h^{+} \pi^{0}$ modes under study. The multiplicity after applying analysis-level selection cuts (summarized in table 2.5) is 1.03 per event for all three decay modes.

The main sources of multiple candidates are:

- Charged track combinatorics in $D^{0} \rightarrow h^{-} h^{+} \pi^{0}$ reconstruction.

- Right $D^{0}$ combined with a fake $\pi_{s}$ to make a $D^{*}$.

- More than one $\pi^{0}$ candidate with or without a shared photon.

- Fake/mis-reconstructed $\pi^{0}$.

In order to select only one candidate per event, it is necessary to define a criterion that permits one to identify, as far as possible, the combination with the largest probability of being a true signal. In events with multiplicity greater than one, we select the candidate with the minimum value of combined vertex $\chi^{2}$, where the vertex $\chi^{2}$ is calculated for the whole decay chain $\left(D^{*+} \rightarrow D^{0} \pi_{s}^{+}, D^{0} \rightarrow h^{-} h^{+} \pi^{0}\right.$, $\pi^{0} \rightarrow \gamma \gamma$ ). We studied the effect of this selection criterion on simulated events and found that our procedure selects the correct candidate $\sim 82 \%$ of the time.

\subsection{Next steps}

At this stage, we have already obtained a relatively clean subset of the data sample and optimized selection criteria to obtain $h^{-} h^{+} \pi^{0}$ signals with greater sensitivity. Now we store the necessary event variables needed for physics analysis, extract the number of signal events, and calculate event reconstruction efficiencies. We describe the physics analysis procedure in detail in the next chapters. 


\section{Chapter 3}

\section{Branching Ratio Measurements of $D^{0} \rightarrow \pi^{-} \pi^{+} \pi^{0}, K^{-} K^{+} \pi^{0}$}

In this chapter we describe the measurement [18] of the relative branching ratios of the three-body singly Cabibbo-suppressed decays $D^{0} \rightarrow \pi^{-} \pi^{+} \pi^{0}$ and $D^{0} \rightarrow$ $K^{-} K^{+} \pi^{0}$ with respect to the Cabibbo-favored decay $D^{0} \rightarrow K^{-} \pi^{+} \pi^{0}$. The relative branching ratios $\frac{\mathcal{B}\left(D^{0} \rightarrow \pi^{-} \pi^{+} \pi^{0}\right)}{\mathcal{B}\left(D^{0} \rightarrow K^{-} \pi^{+} \pi^{0}\right)}$ and $\frac{\mathcal{B}\left(D^{0} \rightarrow K^{-} K^{+} \pi^{0}\right)}{\mathcal{B}\left(D^{0} \rightarrow K^{-} \pi^{+} \pi^{0}\right)}$ are simply the ratios of the reconstructed signal events after being corrected for detector acceptance and reconstruction efficiency

$$
\frac{\mathcal{B}\left(D^{0} \rightarrow \pi^{-} \pi^{+} \pi^{0}\right)}{\mathcal{B}\left(D^{0} \rightarrow K^{-} \pi^{+} \pi^{0}\right)}=\frac{S_{\pi^{-} \pi^{+} \pi^{0}} \times W_{\pi^{-} \pi^{+} \pi^{0}}}{S_{K^{-} \pi^{+} \pi^{0}} \times W_{K^{-} \pi^{+} \pi^{0}}}
$$

and

$$
\frac{\mathcal{B}\left(D^{0} \rightarrow K^{-} K^{+} \pi^{0}\right)}{\mathcal{B}\left(D^{0} \rightarrow K^{-} \pi^{+} \pi^{0}\right)}=\frac{S_{K^{-} K^{+} \pi^{0}} \times W_{K^{-} K^{+} \pi^{0}}}{S_{K^{-} \pi^{+} \pi^{0}} \times W_{K^{-} \pi^{+} \pi^{0}}},
$$

where $\mathrm{S}$ and $W$ denote, respectively, the raw number of signal events detected and the average inverse signal reconstruction efficiency. We obtain the raw number of signal events using the invariant mass distribution of the reconstructed $D^{0}$ candidate and fitting for the signal peak around the nominal $D^{0}$ mass. We estimate signal reconstruction efficiency using simulated events.

\subsection{Description of backgrounds}

For all the three modes under study, there are combinatorial backgrounds normally encountered in any multi-body charm decay events. The sources of this combinatorial background are:

- Charged track combinatorics in $D^{0} \rightarrow h^{-} h^{+} \pi^{0}$ reconstruction.

- Fake/mis-reconstructed $\pi^{0}$.

- Real $D^{0}$, fake $\pi_{s}$. 
Figures 3.1-3.1 show the $\Delta m=m_{D^{*}}-m_{D^{0}}$ distributions for the reconstructed $D^{0}$ events in simulation and data. The combinatorial background is non-peaking and uniform and, therefore, can be modelled by a linear or exponential function. Apart from the combinatorial background, for $\pi^{-} \pi^{+} \pi^{0}$ and $K^{-} K^{+} \pi^{0}$ modes, there is a peaking background in the lower and upper sidebands respectively due to the $K^{-} \pi^{+} \pi^{0}$ reflection (i.e., when the kaon in $K^{-} \pi^{+} \pi^{0}$ is mis-identified as a pion thus peaking in the lower sideband of $\pi^{-} \pi^{+} \pi^{0}$ invariant mass or when the pion in $K^{-} \pi^{+} \pi^{0}$ is mis-identified as a kaon thus peaking this time in the upper sideband of $K^{-} K^{+} \pi^{0}$ invariant mass). The levels of different background contributions in $\pi^{-} \pi^{+} \pi^{0}$ and $K^{-} K^{+} \pi^{0}$ invariant mass are shown in Figure 3.3. The details of this background and its parameterization are described in the next section.

\subsection{Extracting signal events}

To estimate the number of signal events, all of the $h^{-} h^{+} \pi^{0}$ samples are fit using similar procedures. The $D^{0}$ signals are always fit as the sums of three Gaussians. The central values, as well as the widths, of these Gaussians are allowed to vary. All three decay modes have combinatorial backgrounds which are modeled using linear functions. In addition, reflections of misidentified $D^{0} \rightarrow K^{-} \pi^{+} \pi^{0}$ signals are explicitly added to the background descriptions for the $\pi^{-} \pi^{+} \pi^{0}$ and $K^{-} K^{+} \pi^{0}$ samples. In order to estimate the systematic error in the number of signal events due to background modeling, we repeat the $D^{0}$ mass fit with exponential and polynomial function for combinatorial background.

The $\pi^{-} \pi^{+} \pi^{0}$ samples have background from $D^{0} \rightarrow K^{-} \pi^{+} \pi^{0}$ decays in which the kaon is misidentified as a pion. To determine the shape of this reflection, Monte Carlo signal events of $D^{0} \rightarrow K^{-} \pi^{+} \pi^{0}$ are reconstructed as $\pi^{-} \pi^{+} \pi^{0}$. The reflected invariant mass distribution of these events is shown in Figure 3.3. Figure 3.4 shows the shape and number of $K^{-} \pi^{+} \pi^{0}$ reflection events in the $\pi^{-} \pi^{+} \pi^{0}$ reconstruction. The shape is parameterized using a sum of three Gaussians having different means and widths (there is no physical significance to this particular parameterization, we simply used a PDF which fits the reflection peak well). The level of $K^{-} \pi^{+} \pi^{0}$ reflection in $\pi^{-} \pi^{+} \pi^{0}$ data sample is determined by fitting the distribution of these mis-identified data events using the $K^{-} \pi^{+} \pi^{0}$ hypothesis. The peak at the $D^{0}$ mass clearly arises from reflection, and its area determines the level of this contamination. The reflection contribution to the $\pi^{-} \pi^{+} \pi^{0}$ mass fit is fixed by this level and by the shape determined from Monte Carlo.

The $K^{-} K^{+} \pi^{0}$ samples have background from $D^{0} \rightarrow K^{-} \pi^{+} \pi^{0}$ decays in which the pion is misidentified as a kaon. To determine the shape of this reflection, Monte Carlo signal events of $D^{0} \rightarrow K^{-} \pi^{+} \pi^{0}$ are reconstructed as $K^{-} K^{+} \pi^{0}$. Figure 3.5 shows the shape and number of $K^{-} \pi^{+} \pi^{0}$ reflection events in the $K^{-} K^{+} \pi^{0}$ reconstruction. Again the shape is parameterized using a sum of three Gaussians having different means and widths. The level of $K^{-} \pi^{+} \pi^{0}$ reflection in $K^{-} K^{+} \pi^{0}$ data sample is determined by fitting the distribution of these reflection events using the $K^{-} \pi^{+} \pi^{0}$ hypothesis. 

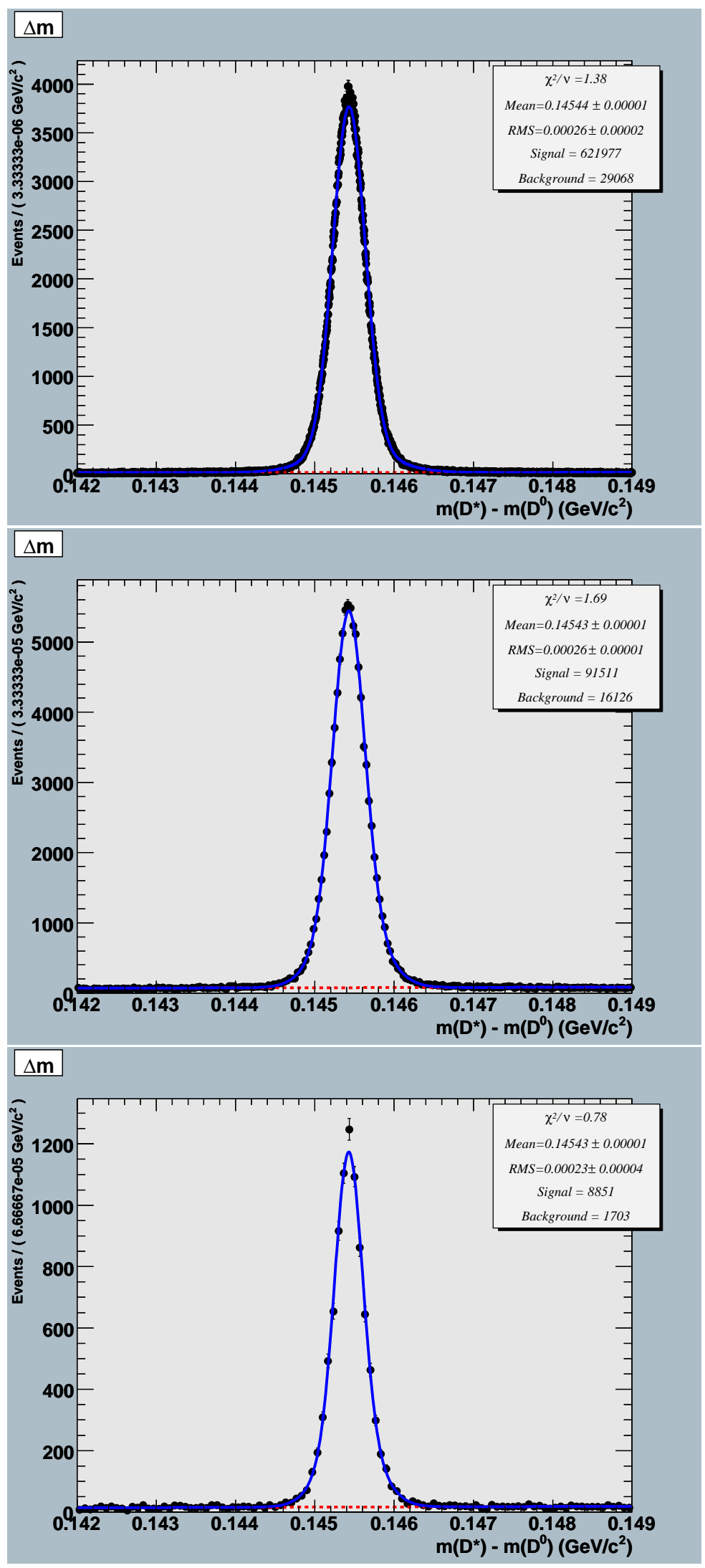

Figure 3.1: $\Delta m=m_{D^{*}}-m_{D^{0}}$ distributions for $K^{-} \pi^{+} \pi^{0}$ (top), $\pi^{-} \pi^{+} \pi^{0}$ (middle), and $K^{-} K^{+} \pi^{0}$ (bottom) generic $c \bar{c}$ simulated events around $\pm 1 \sigma$ of the $D^{0}$ invariant mass. 

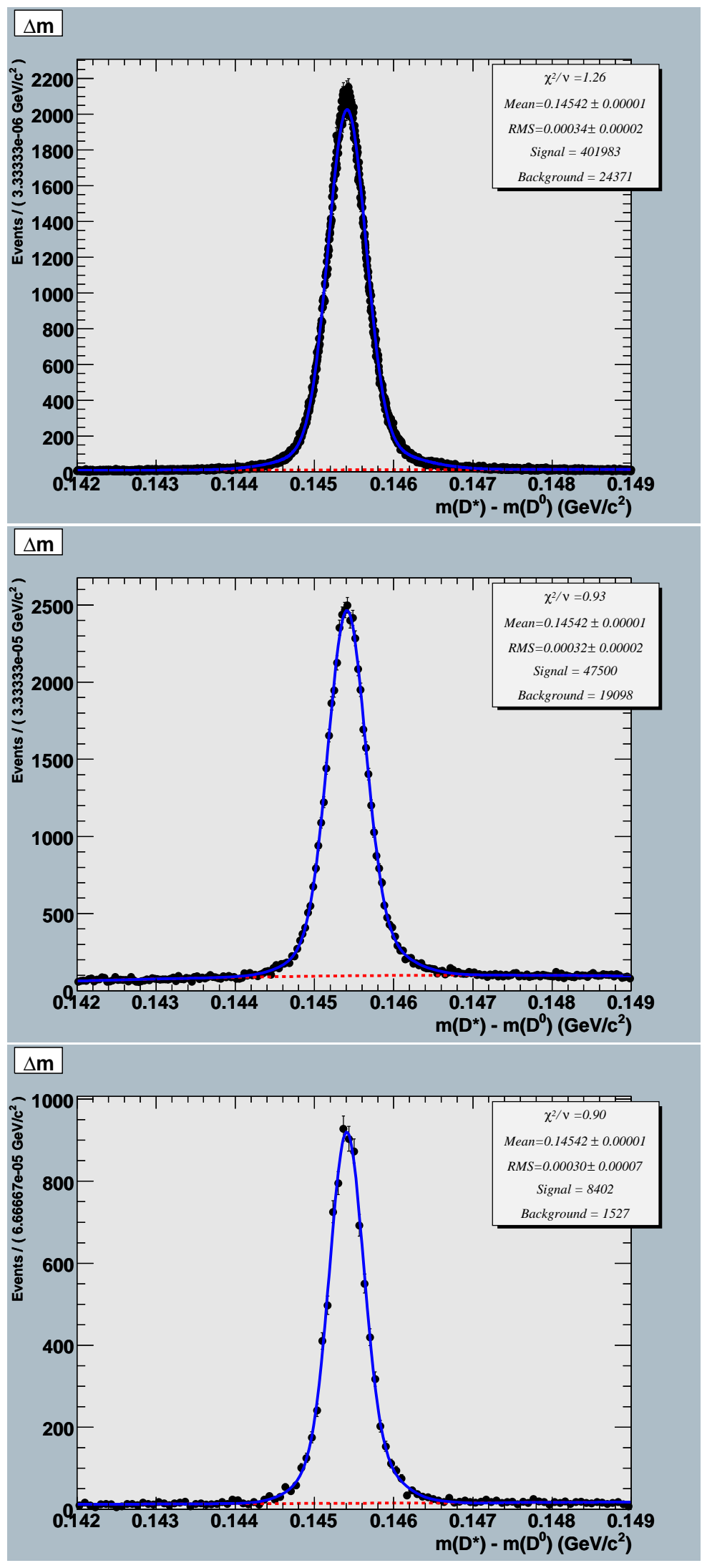

Figure 3.2: $\Delta m=m_{D^{*}}-m_{D^{0}}$ distributions for $K^{-} \pi^{+} \pi^{0}$ (top), $\pi^{-} \pi^{+} \pi^{0}$ (middle), and $K^{-} K^{+} \pi^{0}$ (bottom) data events arouß3d $\pm 1 \sigma$ of the $D^{0}$ invariant mass. 

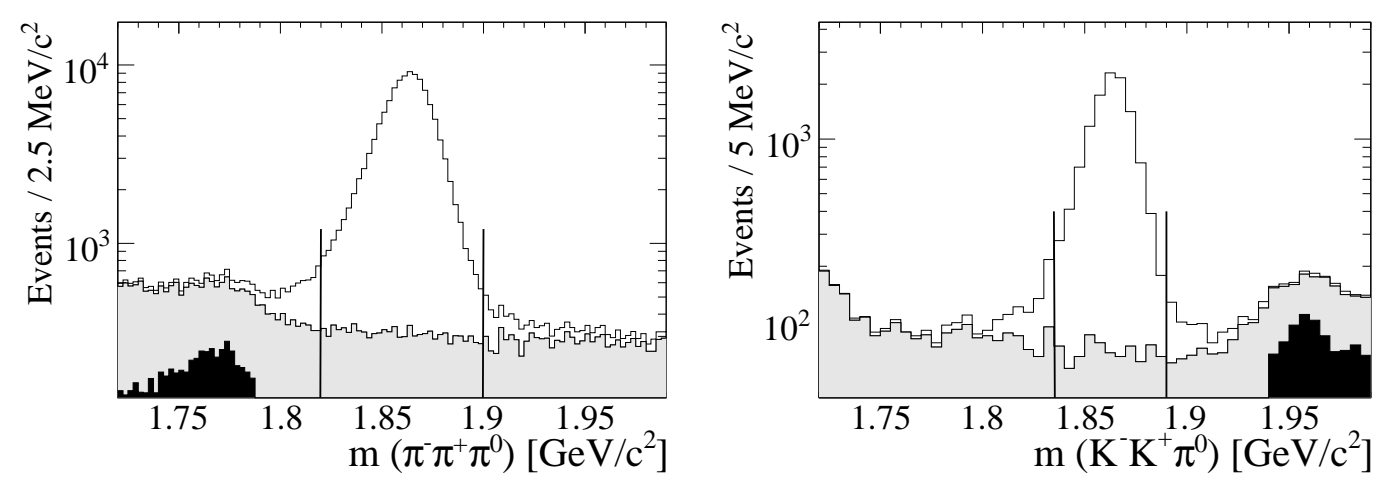

Figure 3.3: Simulated $\pi^{-} \pi^{+} \pi^{0}$ (left) and $K^{-} K^{+} \pi^{0}$ (right) invariant mass distributions. Signal events are shown as open histograms. Combinatorial and reflection backgrounds are shown by the light and dark shaded histograms, respectively. The signal region is delimited by the vertical lines.
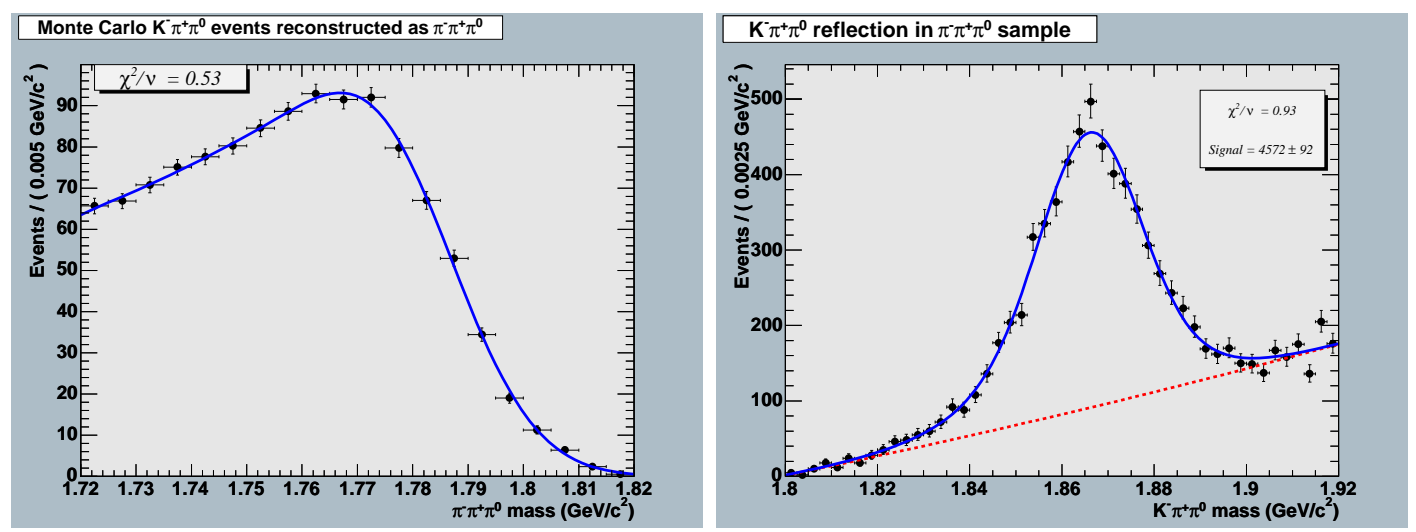

Figure 3.4: Simulated $K^{-} \pi^{+} \pi^{0}$ events reconstructed as $\pi^{-} \pi^{+} \pi^{0}$ (left) and the level of this reflection in $\pi^{-} \pi^{+} \pi^{0}$ data sample (right).
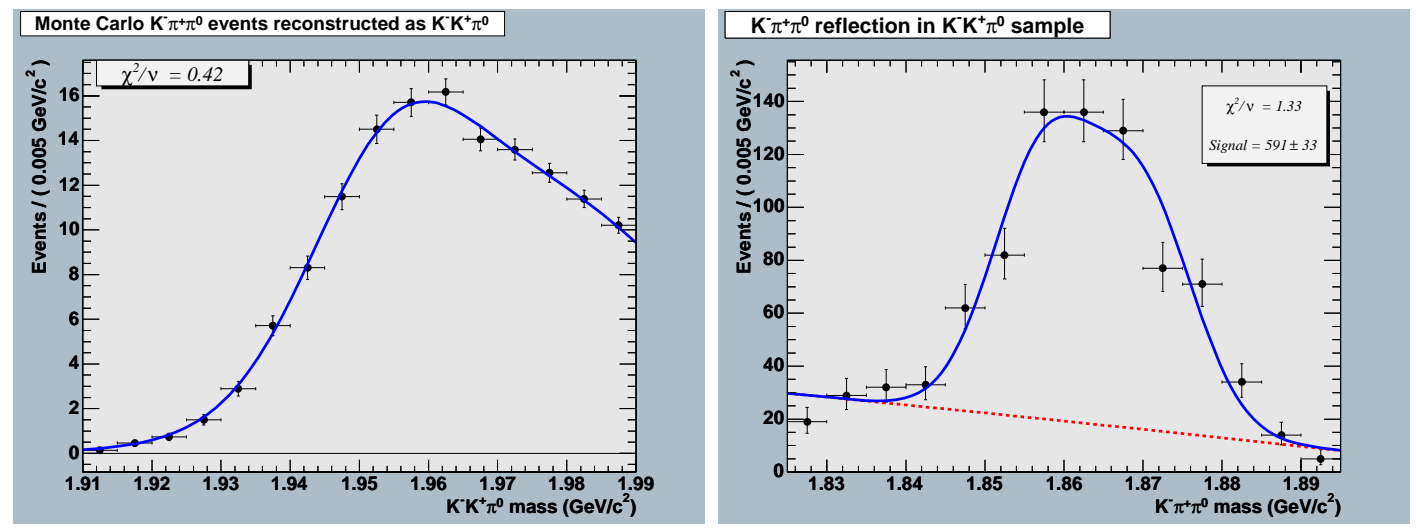

Figure 3.5: Simulated $K^{-} \pi^{+} \pi^{0}$ events reconstructed as $K^{-} K^{+} \pi^{0}$ (left) and the level of this reflection in $K^{-} K^{+} \pi^{0}$ data sample (right). 

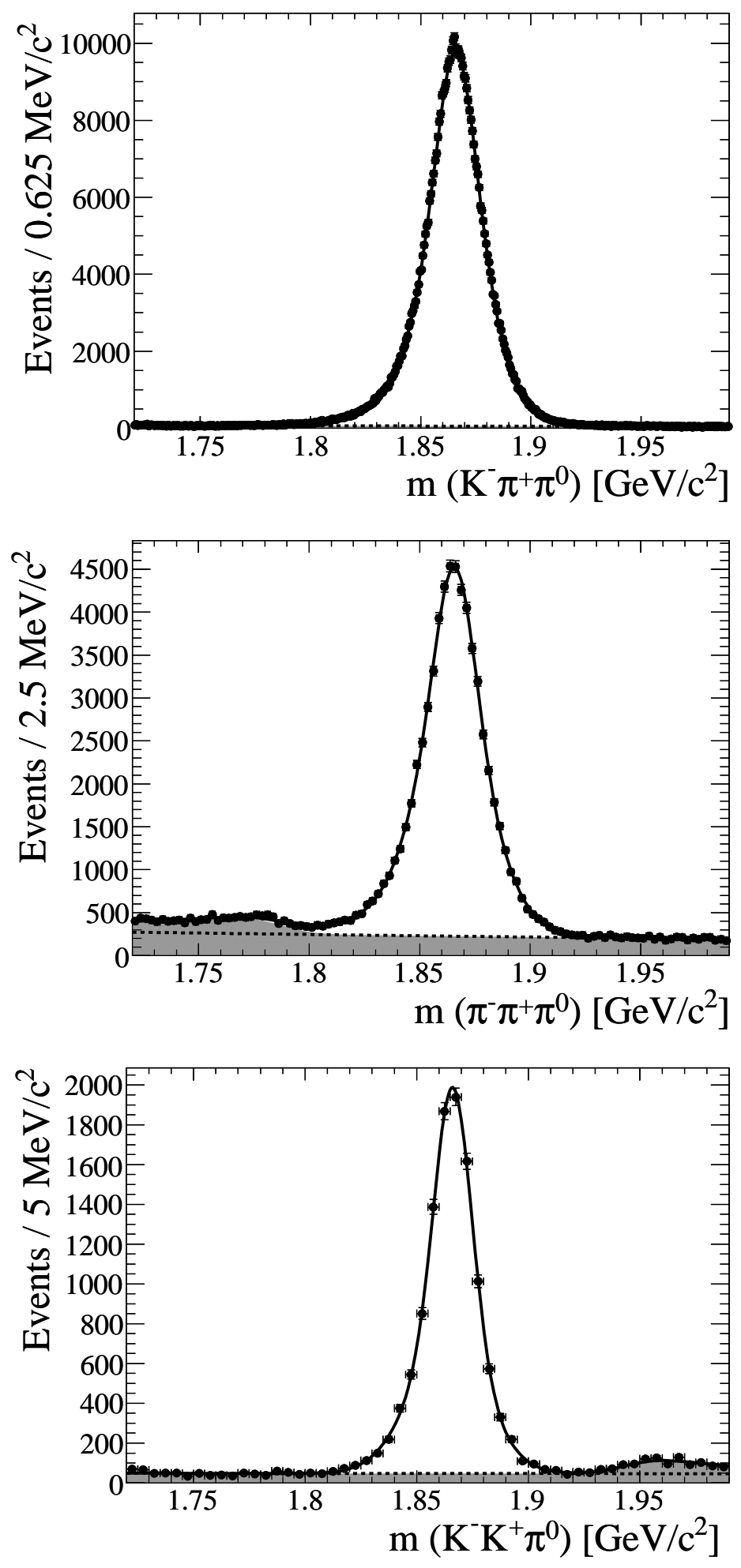

Figure 3.6: Fitted mass for the $K^{-} \pi^{+} \pi^{0}, \pi^{-} \pi^{+} \pi^{0}$, and $K^{-} K^{+} \pi^{0}$ data samples. Dots are data points and the solid curves are the fit. The dot-dashed lines show the level of combinatorial background in each case. For the $\pi^{-} \pi^{+} \pi^{0}$ and the $K^{-} K^{+} \pi^{0}$ modes, the shaded region represents the total background. 


\begin{tabular}{|c|c|c|}
\hline Mode & Mean $\left[\mathrm{GeV} / c^{2}\right]$ & $R M S\left[\mathrm{GeV} / c^{2}\right]$ \\
\hline \hline$K^{-} \pi^{+} \pi^{0}$ data & $1.8646 \pm 0.0002$ & $0.0160 \pm 0.0005$ \\
\hline$K^{-} \pi^{+} \pi^{0}$ simulation & $1.8615 \pm 0.0001$ & $0.0153 \pm 0.0005$ \\
\hline$\pi^{-} \pi^{+} \pi^{0}$ data & $1.8637 \pm 0.0004$ & $0.0174 \pm 0.0008$ \\
\hline$\pi^{-} \pi^{+} \pi^{0}$ simulation & $1.8609 \pm 0.0002$ & $0.0150 \pm 0.0009$ \\
\hline$K^{-} K^{+} \pi^{0}$ data & $1.8649 \pm 0.0004$ & $0.0135 \pm 0.0010$ \\
\hline$K^{-} K^{+} \pi^{0}$ simulation & $1.8624 \pm 0.0002$ & $0.0086 \pm 0.0009$ \\
\hline
\end{tabular}

Table 3.1: Mean and RMS values of $D^{0}$ candidate invariant mass distribution with both signal and some background.

\begin{tabular}{|c|c|c|c|}
\hline Mode & Signal & Mass & Width \\
\hline$\pi^{-} \pi^{+} \pi^{0}$ & $60964 \pm 341$ & $1.8637 \pm 0.0004$ & $0.0174 \pm 0.0008$ \\
\hline$K^{-} \pi^{+} \pi^{0}$ & $505660 \pm 750$ & $1.8646 \pm 0.0002$ & $0.0160 \pm 0.0005$ \\
\hline$K^{-} K^{+} \pi^{0}$ & $10773 \pm 122$ & $1.8649 \pm 0.0004$ & $0.0135 \pm 0.0010$ \\
\hline
\end{tabular}

Table 3.2: A summary of the number of observed events and other fit results for the reconstructed $D^{0}$ invariant mass.

\subsection{Signal reconstruction efficiency}

We estimate signal efficiency for each event as a function of its position in the Dalitz plot [19] using simulated $D^{0} \rightarrow h^{-} h^{+} \pi^{0}$ events from $c \bar{c}$ decays, generated uniformly in the available phase space and subjected to the same reconstruction and selection procedure applied to data. To correct for differences in particle-identification rates in data and simulation, we determine the ratio of these for each track, and apply an event-by-event correction factor.

We parametrize this efficiency with a 3rd order polynomial shape in two dimensions in terms of squared invariant masses $m^{2}\left(h^{-} \pi^{0}\right)$ and $m^{2}\left(h^{+} \pi^{0}\right)$. The signal reconstruction efficiencies for each of the three $h^{-} h^{+} \pi^{0}$ decays are shown in Figure 3.7. The Dalitz plots for real data events for $h^{-} h^{+} \pi^{0}$ data samples are shown in Figure 3.3.

We use the observed bin-by-bin efficiency for applying the efficiency-correction to data events. The parametrized efficiency serves as a cross-check. We calculate the efficiency for each real data signal event and take the inverse of the efficiency as the event's weight. The average weight for each decay mode is computed by summing the weights of events in the signal region and subtracting scaled weights from sidebands to account for background events in the signal region. Signal data events are selected in the mass region of $\pm 3 \mathrm{rms}$ width from the central value. Background under the signal is subtracted selecting events from the sidebands. For $K^{-} \pi^{+} \pi^{0}$ mode, we use two side bands, $1.73<M\left(h^{-} h^{+} \pi^{0}\right)<1.77 \mathrm{GeV} / c^{2}$ and $1.95<M\left(h^{-} h^{+} \pi^{0}\right)<1.99 \mathrm{GeV} / c^{2}$ which are almost symmetrically spaced around the $D^{0}$ mass. For $\pi^{-} \pi^{+} \pi^{0}$ mode we use the upper sideband $\left(1.93<M\left(h^{-} h^{+} \pi^{0}\right)<\right.$ $\left.1.99 \mathrm{GeV} / c^{2}\right)$ only because of the presence of $K^{-} \pi^{+} \pi^{0}$ reflection events in the lower sideband. Similarly, we use lower sideband $\left(1.75<M\left(h^{-} h^{+} \pi^{0}\right)<1.80 \mathrm{GeV} / c^{2}\right)$ for the $K^{-} K^{+} \pi^{0}$ mode because of the presence of $K^{-} \pi^{+} \pi^{0}$ reflection events in the 
Efficiency [\%] across K $\pi^{+} \pi^{0}$ Dalitz plot-data

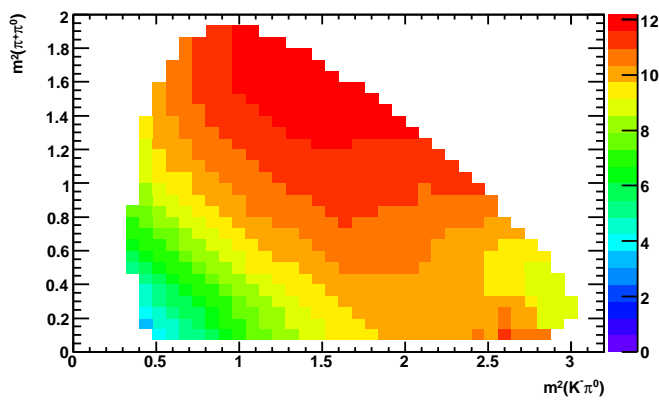

Efficiency [\%] across $\pi^{2} \pi^{+} \pi^{0}$ Dalitz plot-data

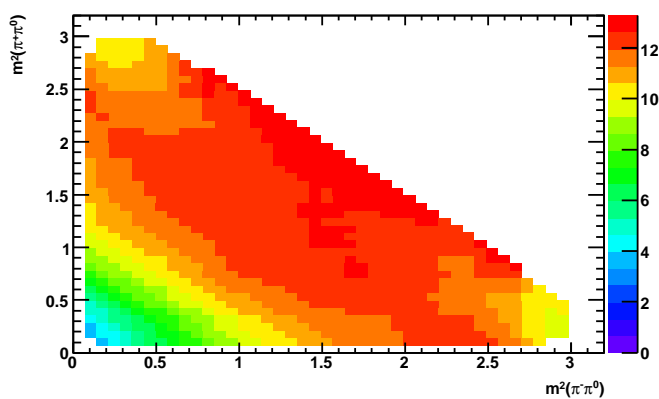

Efficiency [\%] across $\mathrm{KK}^{+} \pi^{0}$ Dalitz plot-data

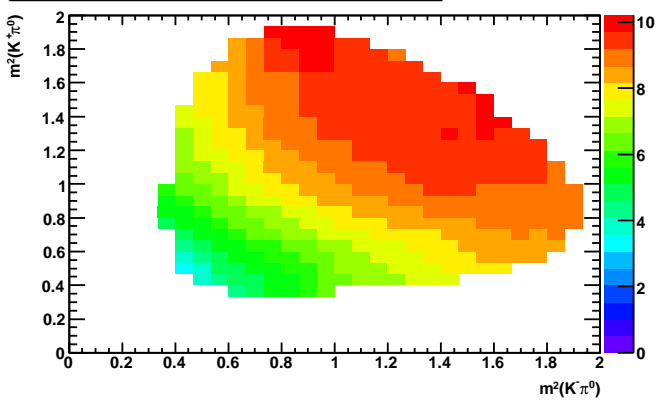

Efficiency [\%] across K $\pi^{+} \pi^{0}$ Dalitz plot - fit

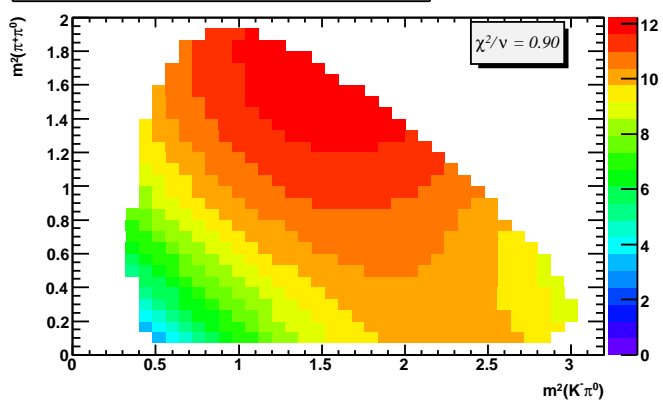

Efficiency [\%] across $\pi^{*} \pi^{+} \pi^{0}$ Dalitz plot - fit

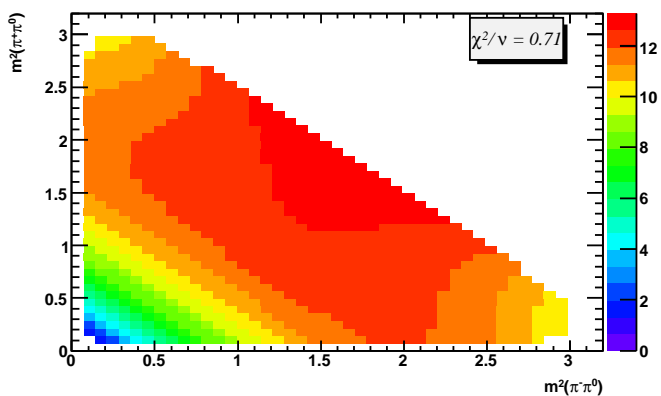

Efficiency [\%] across $\mathrm{KK}^{+} \pi^{0}$ Dalitz plot - fit

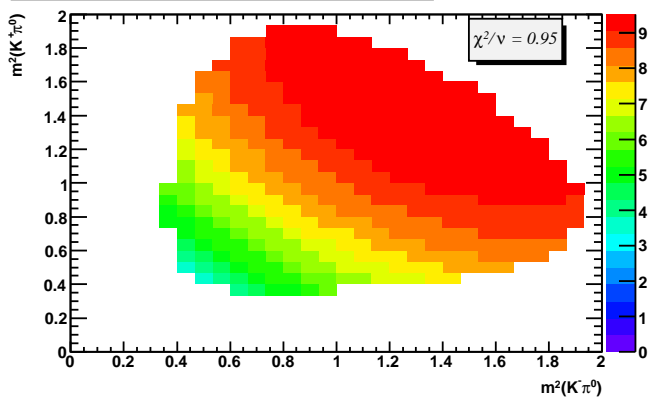

Figure 3.7: Efficiency across Dalitz plots for $K^{-} \pi^{+} \pi^{0}, \pi^{-} \pi^{+} \pi^{0}$, and $K^{-} K^{+} \pi^{0}$ signal reconstruction obtained using simulated events from $c \bar{c}$ decays, generated uniformly in the available phase space. The observed efficienciencies are shown on the left and the parametrized ones (using a 3rd order polynomial) are shown on the right. 
upper sideband. The contribution from the sideband is scaled to the area of the background shape in the signal region.

\subsection{Number of signal events produced}

The number of signal events produced in the experiment is

$$
S_{\text {produced }}=\sum\left(\frac{1}{\epsilon_{i}}\right)
$$

where the sum is taken over observed signal events and $\epsilon_{i}$ is the overall reconstruction efficiency for each of those events. We cannot separate signal and background events in our final sample event by event, so we estimate an average value of $1 / \epsilon$ for signal events in each sample and use these to weight the observed signals; see Eqns. 3.1 and 3.2. The average weight for each sample depends upon the distribution of signal events in the Dalitz plot and upon the efficiency determined from the Monte Carlo simulation. The average weight for signal events in the signal region is calculated by summing the weights of all the events in a signal region and subtracting the scaled weights of events in the sideband to account for the weights of background events in the signal region, and then dividing by the number of signal events, as described in previous sections. Therefore, the number of signal events produced in the experiment for each mode, is the product of the number of signal events obtained from the fit to the $D^{0}$ candidate mass (scaled to the area of the signal region, i.e., within 3 RMS width from the central value) and the average weight of signal events.

$$
S_{\text {produced }}=S_{\text {SignalRegion }} \times<W>
$$

\subsection{Calculation of ratios of branching ratios}

Table 3.3 summarizes the numbers used to calculate the central values of the ratios of branching ratios, the statistical errors, and part of the systematic errors. The second column reports the number of signal events detected in each decay mode along with statistical error in them, as reported earlier in Table 3.2. The third column reports the average inverse efficiency after correcting for difference in particle-identification rates in data and simulation.

\begin{tabular}{|c|c|c|c|}
\hline Mode & Signal $(S)$ & ave.weight $(<W>)$ & ave.weight from fit \\
\hline \hline$\pi^{-} \pi^{+} \pi^{0}$ & $60964 \pm 341$ & $9.4308 \pm 0.0198$ & $9.4485 \pm 0.0194$ \\
\hline$K^{-} \pi^{+} \pi^{0}$ & $505660 \pm 750$ & $10.7471 \pm 0.0182$ & $10.7366 \pm 0.0187$ \\
\hline$K^{-} K^{+} \pi^{0}$ & $10773 \pm 122$ & $12.6101 \pm 0.0530$ & $12.6312 \pm 0.0551$ \\
\hline
\end{tabular}

Table 3.3: Final yield and average weight and errors associated with them.

\subsection{Study of systematic uncertainties}

We investigate the following sources of systematic uncertainty in the measurement of ratios of branching ratios: 

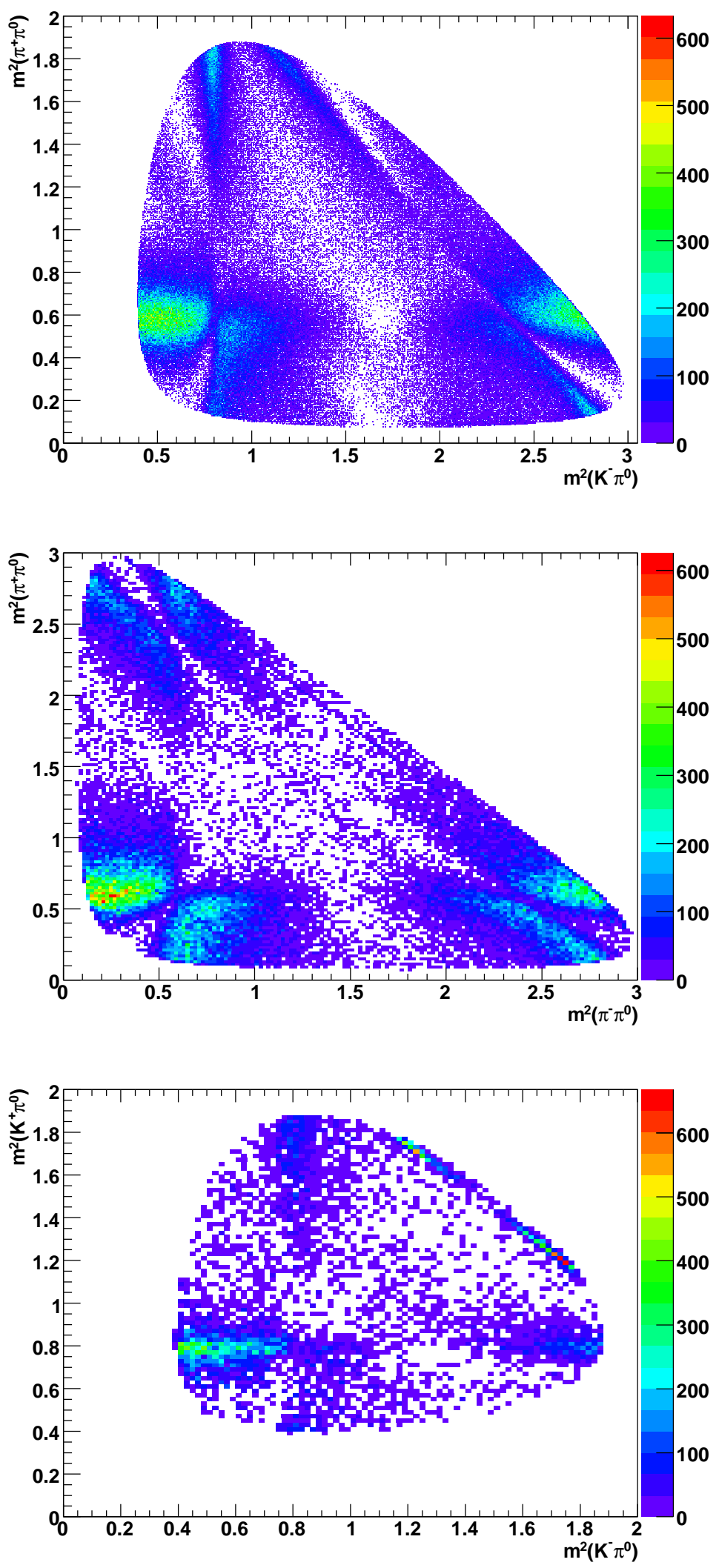

Figure 3.8: Efficiency corrected $K^{-} \pi^{+} \pi^{0}, \pi^{-} \pi^{+} \pi^{0}$, and $K^{-} K^{+} \pi^{0}$ Dalitz plots. 
- Uncertainty due to finite size of the simulated data sample which is used to determine signal reconstruction efficiency.

- Variation in signal optimization criteria ( $\Delta m$ selection)

- Error from estimation of background events in signal region

- Effect of background shape on signal yield

- Effect of difference in $D^{0} \mathrm{CM}$ momentum distribution in data and simulation

- Systematic uncertainty from particle identification

- Tracking efficiency systematics

Also, as consistency check, we did the analysis seperately for $D^{0}$ and $\bar{D}^{0}$ events in different $D^{0}$ candidate lab momentum ranges to look for systematic variations as a function of charge or momentum outside the levels accounted for in our estimates of statistical and systematic uncertainties. Each of them are described in detail in the following sections.

\subsection{Systematic error in average weight}

There are two sources of systematic error in the average weight obtained by above method:

- the uncertainty in event reconstruction efficiency due to limited statistics of the Monte Carlo sample.

- the uncertainty due to the assumption that the average weight of background events in the signal region is the same as the average weight of background events in the sideband.

\subsubsection{Systematic error due to simulation statistics}

We estimate the systematic error in the average weight in the following way. In each bin of the Dalitz plot we assign an efficiency by generating a random number with Gaussian distribution having the following parameters:

- mean $=$ number of events reconstructed in a particular bin / number of events generated in that bin

- width $=$ fractional error in the above ratio

Now, we calculate the average weight using this new efficiency. We repeat this process several times and take the average of all these measurements. The deviation of this value from the originally obtained average weight represents the systematic error in the average weight. 


\subsubsection{Systematic error from background subtraction}

First of all, we did a feasibility study to find out whether the background events in the signal region and the sideband have the same Dalitz plot distribution or not. We proceeded in four steps:

- First, we compared the Dalitz plot distributions for events in the sidebands of $m_{D^{0}}$ in real data and in generic $q \bar{q}$ simulation. For this purpose, we use only the upper sideband of $D^{0} \rightarrow \pi^{-} \pi^{+} \pi^{0}$ and the lower sideband of $D^{0} \rightarrow$ $K^{-} K^{+} \pi^{0}$ events, because of contamination from $D^{0} \rightarrow K^{-} \pi^{+} \pi^{0}$ events in the lower (upper) sideband of $D^{0} \rightarrow \pi^{-} \pi^{+} \pi^{0}\left(D^{0} \rightarrow K^{-} K^{+} \pi^{0}\right)$. Figures 3.9 and 3.10 show the normalized residuals of these two distributions for $\pi^{-} \pi^{+} \pi^{0}$ and $K^{-} K^{+} \pi^{0}$ decays respectively. The agreement between the Dalitz plot distributions in the sidebands of data and simulation is good.
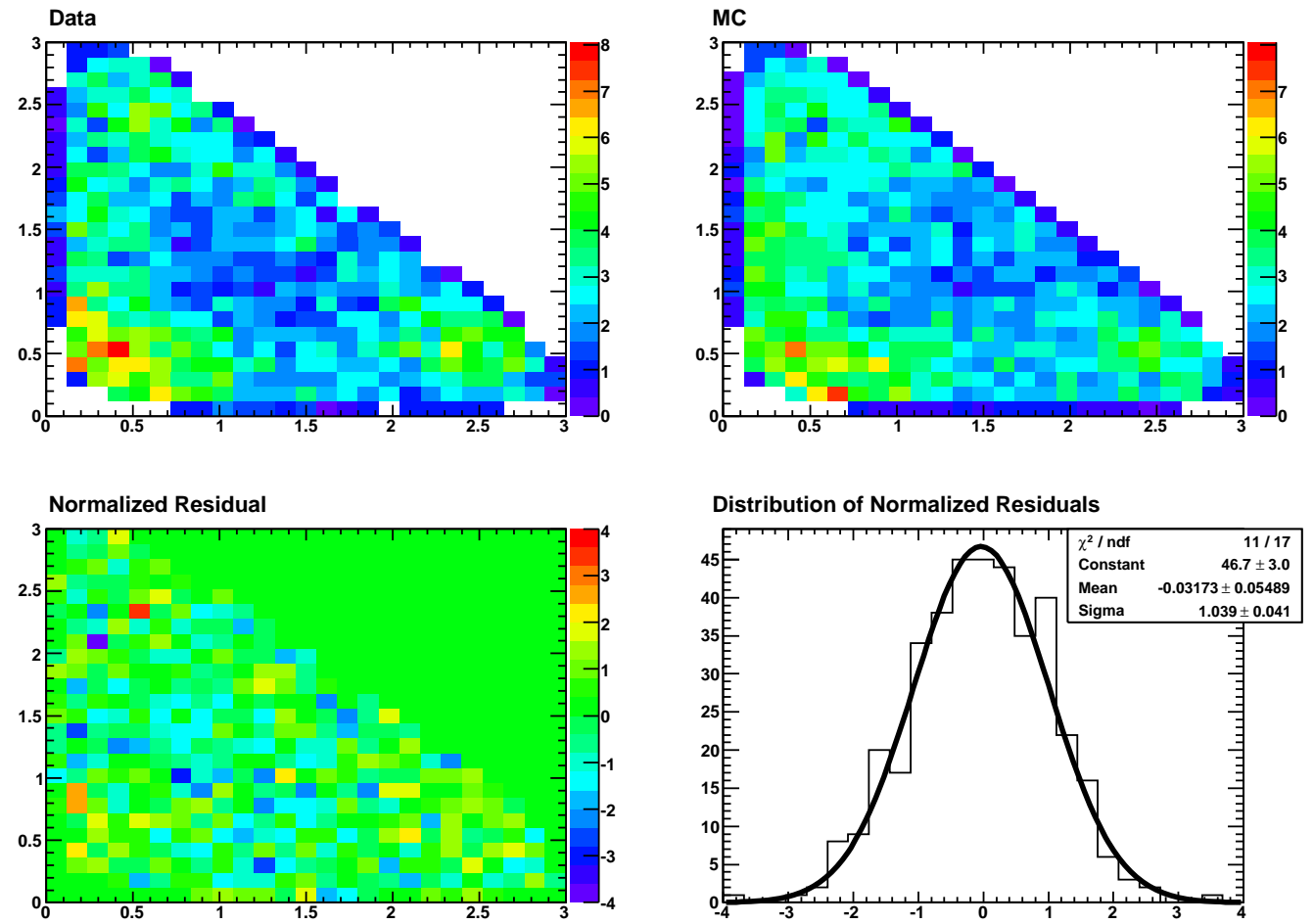

Figure 3.9: Comparison of $D^{0} \rightarrow \pi^{-} \pi^{+} \pi^{0}$ Dalitz plot distributions for events in the upper sideband of $m_{D^{0}}$ in data and simulation.

- Next, we compared the Dalitz plot distributions of the background events in the signal region and the sideband for generic $q \bar{q}$ simulated events. The normalized residuals of these two distributions are shown in Figures 3.11 and 3.12 for $\pi^{-} \pi^{+} \pi^{0}$ and $K^{-} K^{+} \pi^{0}$ decays respectively. Again the agreement between data and simulation is very good. 

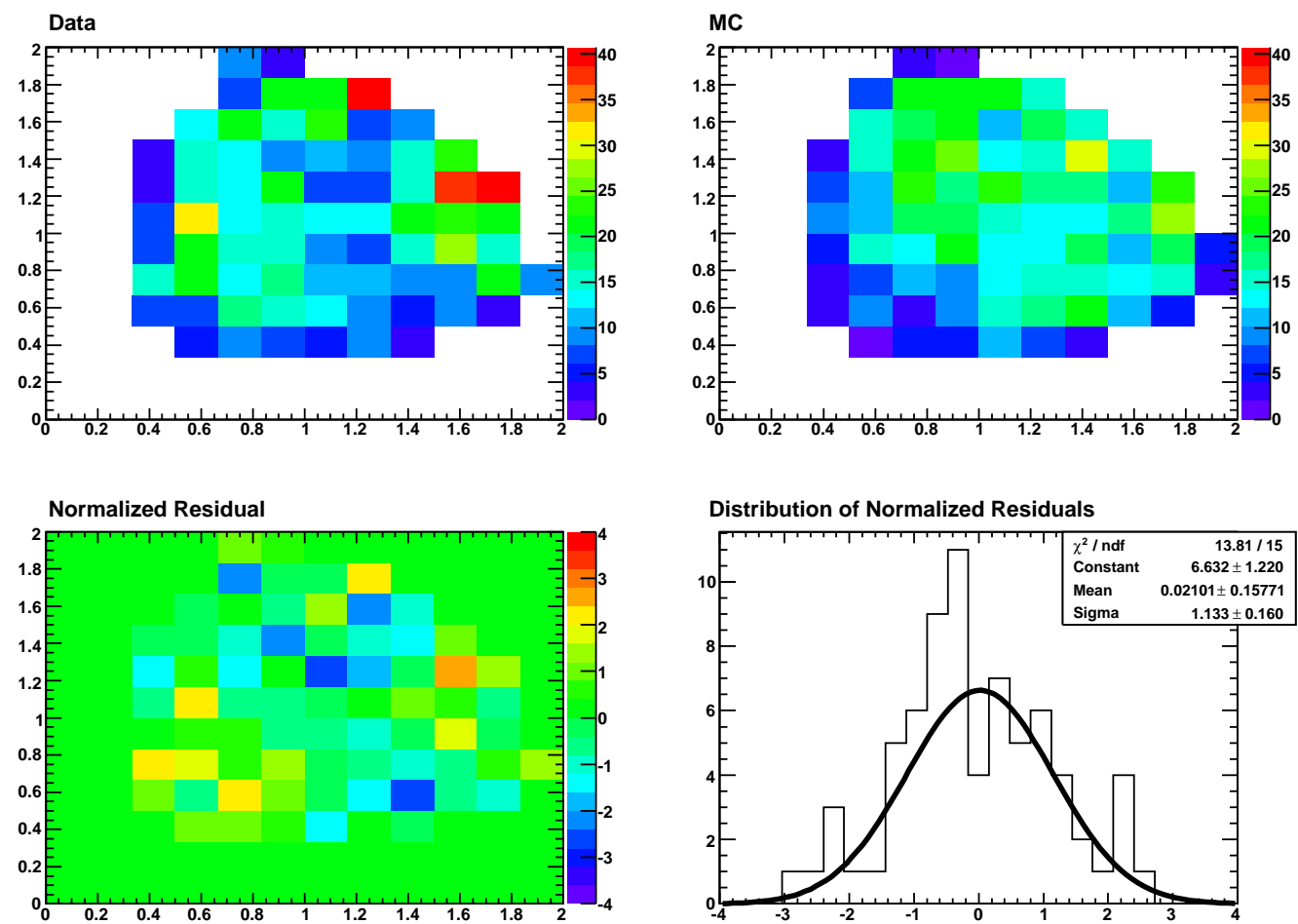

Figure 3.10: Comparison of $D^{0} \rightarrow K^{-} K^{+} \pi^{0}$ Dalitz plot distributions for events in the lower sideband of $m_{D^{0}}$ in data and simulation. 

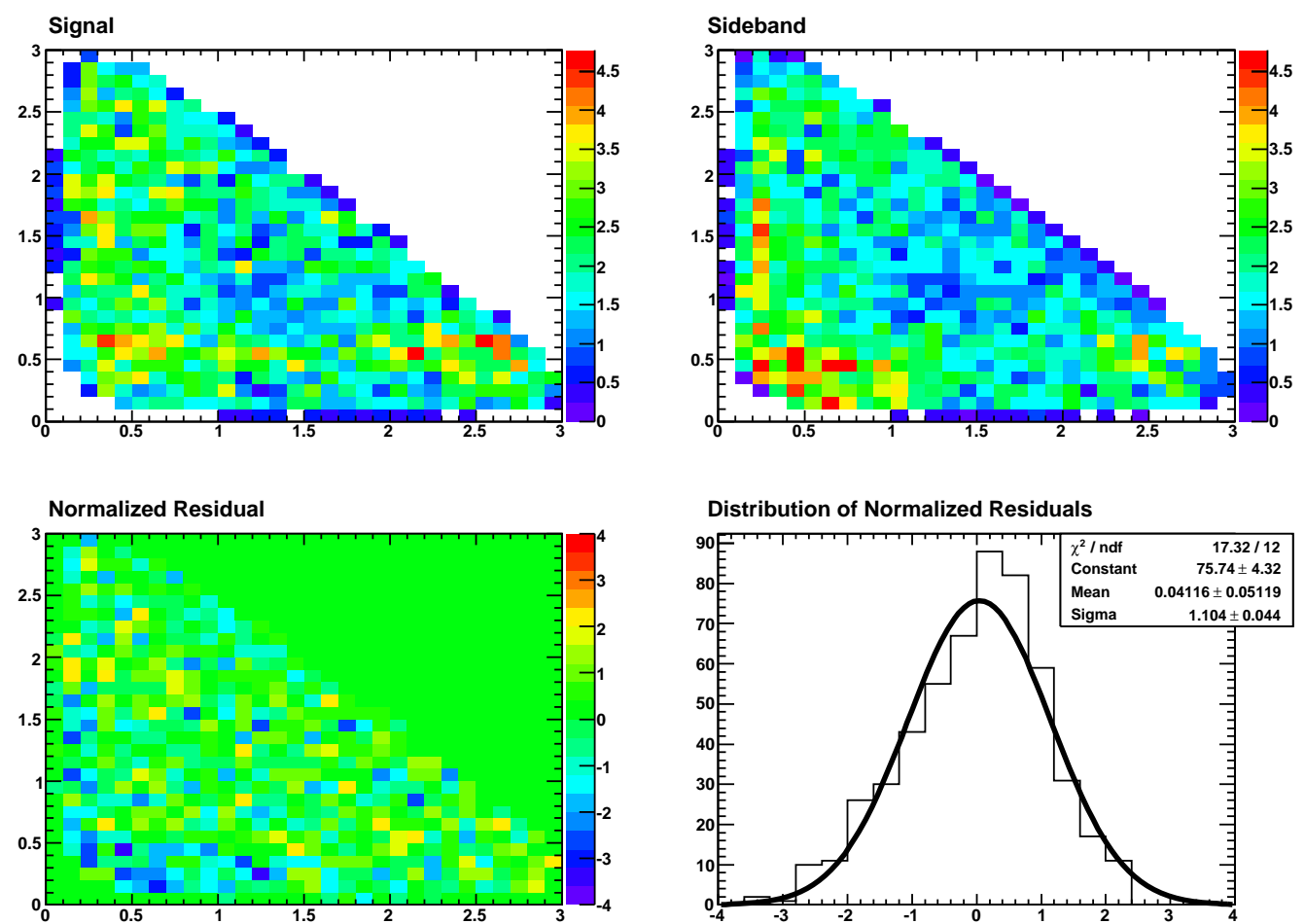

Figure 3.11: Comparison of $D^{0} \rightarrow \pi^{-} \pi^{+} \pi^{0}$ Dalitz plot distributions for events in the signal region and in the upper sideband of $m_{D^{0}}$ in simulation. 

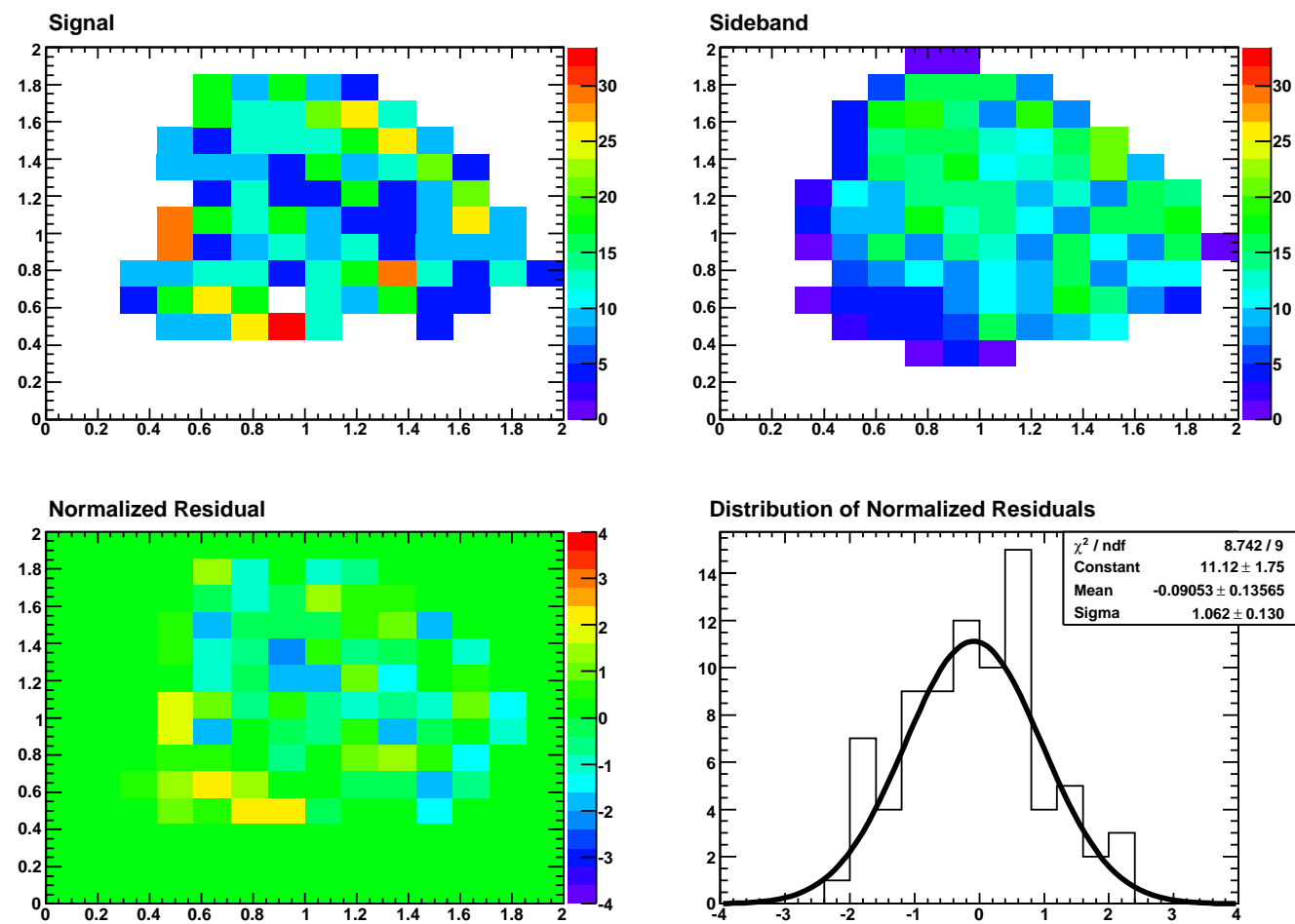

Figure 3.12: Comparison of $D^{0} \rightarrow K^{-} K^{+} \pi^{0}$ Dalitz plot distributions for events in the signal region and in the lower sideband of $m_{D^{0}}$ in simulation. 
- We also did a comparison study of $D^{0}$ candidate mass and Dalitz plot distributions for truth-matched and non-truth-matched Monte Carlo signal events to make sure that they have very similar distributions.

- Finally, by repeating the whole analysis on generic $c \bar{c}$ events and doing the background subtraction of true background events in the signal region, we find a systematic error of $0.6 \%$ for $\frac{\mathcal{B}\left(D^{0} \rightarrow \pi^{-} \pi^{+} \pi^{0}\right)}{\mathcal{B}\left(D^{0} \rightarrow K^{-} \pi^{+} \pi^{0}\right)}$ and $0.9 \%$ for $\frac{\mathcal{B}\left(D^{0} \rightarrow K^{-} K^{+} \pi^{0}\right)}{\mathcal{B}\left(D^{0} \rightarrow K^{-} \pi^{+} \pi^{0}\right)}$.

\section{8 $\Delta m$ systematics}

As described earlier (in section Optimization of Analysis Cuts), we select only those $D^{*+} \rightarrow D^{0} \pi_{s}^{+}$events which are in the $\Delta m\left(\mathrm{~m}\left(D^{*}\right)-\mathrm{m}\left(D^{0}\right)\right)$ window of $0.6 \mathrm{MeV} / c^{2}$ from the central value. In order to estimate systematic uncertainty due to this selection, we repeated the whole analysis for the $\Delta m$ window of $0.4 \mathrm{MeV} / c^{2}$ and 0.8 $\mathrm{MeV} / c^{2}$ which correspond to roughly $1.5 \sigma$ and $3 \sigma$ respectively of the $\Delta m$ resolution. The results are summarized in Table 3.4.

Based on this study, we assign a systematic uncertainty of $0.3 \%$ in $\frac{\mathcal{B}\left(D^{0} \rightarrow \pi^{-} \pi^{+} \pi^{0}\right)}{\mathcal{B}\left(D^{0} \rightarrow K^{-} \pi^{+} \pi^{0}\right)}$ and $0.9 \%$ in $\frac{\mathcal{B}\left(D^{0} \rightarrow K^{-} K^{+} \pi^{0}\right)}{\mathcal{B}\left(D^{0} \rightarrow K^{-} \pi^{+} \pi^{0}\right)}$ measurements.

\begin{tabular}{|c|c|c|c|}
\hline Ratio & $\Delta m$ cut $=0.4 \mathrm{MeV} / c^{2}$ & $\Delta m$ cut $=0.6 \mathrm{MeV} / c^{2}$ & $\Delta m$ cut $=0.8 \mathrm{MeV} / c^{2}$ \\
\hline \hline$\frac{\mathcal{B}\left(D^{0} \rightarrow \pi^{-} \pi^{+} \pi^{0}\right)}{\mathcal{B}\left(D^{0} \rightarrow K^{-} \pi^{+} \pi^{0}\right)}$ & $0.1065 \pm 0.0006$ & $0.1064 \pm 0.0006$ & $0.1061 \pm 0.0006$ \\
\hline$\frac{\mathcal{B}\left(D^{0} \rightarrow K^{-} K^{+} \pi^{0}\right)}{\mathcal{B}\left(D^{0} \rightarrow K^{-} \pi^{+} \pi^{0}\right)}$ & $0.0236 \pm 0.0004$ & $0.0238 \pm 0.0004$ & $0.0238 \pm 0.0004$ \\
\hline
\end{tabular}

Table 3.4: Results for different $\Delta m$ cuts.

\subsection{Effect of background model on signal yield}

We performed the mass plot fits with different combinatorial background models (linear, polynomial, and exponential shapes). We assign half the maximum deviation in the signal yield (with respect to the default linear background) as the systematic uncertainty in the number of signal events. We find systematic uncertainty of $0.014 \%$ in $K^{-} \pi^{+} \pi^{0}, 0.157 \%$ in $\pi^{-} \pi^{+} \pi^{0}$, and $0.125 \%$ in $K^{-} K^{+} \pi^{0}$ signal yields. Accordingly, we assign a systematics of $0.16 \%$ to $\frac{\mathcal{B}\left(D^{0} \rightarrow \pi^{-} \pi^{+} \pi^{0}\right)}{\mathcal{B}\left(D^{0} \rightarrow K^{-} \pi^{+} \pi^{0}\right)}$ and $0.13 \%$ to $\frac{\mathcal{B}\left(D^{0} \rightarrow K^{-} K^{+} \pi^{0}\right)}{\mathcal{B}\left(D^{0} \rightarrow K^{-} \pi^{+} \pi^{0}\right)}$ due to background modeling.

\subsection{Effect of difference in $P^{*}$ distributions in data and simulation}

The $D^{0}$ candidate momentum in the event's CM frame $\left(P^{*}\right)$ has slightly different distribution in data than in simulation (used to obtain reconstruction efficiency). In order to estimate the uncertainty due to this difference, we correct the reconstruction efficiency by the $P_{\text {data }}^{*} / P_{\mathrm{MC}}^{*}$ ratio event-by-event and obtain average inverse efficiency for the three decay modes again. With this correction, we find that the average inverse efficiency goes down by $0.31 \%$ for the $K^{-} \pi^{+} \pi^{0}, 0.79 \%$ for the $\pi^{-} \pi^{+} \pi^{0}$, 
and $0.27 \%$ for the $K^{-} K^{+} \pi^{0}$ decays. We use this corrected average inverse efficiency in the calculation of central value of our result and assign half the difference as systematics.

Accordingly, we assign systematic uncertainty of $0.24 \%$ to $\frac{\mathcal{B}\left(D^{0} \rightarrow \pi^{-} \pi^{+} \pi^{0}\right)}{\mathcal{B}\left(D^{0} \rightarrow K^{-} \pi^{+} \pi^{0}\right)}$ and $0.02 \%$ to $\frac{\mathcal{B}\left(D^{0} \rightarrow K^{-} K^{+} \pi^{0}\right)}{\mathcal{B}\left(D^{0} \rightarrow K^{-} \pi^{+} \pi^{0}\right)}$ due to different $P^{*}$ distributions in data and simulation.

\subsection{Particle-identification correction and systematics}

The systematic uncertainty due to difference in particle-identification efficiencies in data and simulation mostly cancel out in the ratio of Branching Ratios. There is a small residual uncertainty though as explained below.

There are two components two this systematics:

- The systematic errors in kaon and pion identification efficiencies and misidentification rates.

- The systematic uncertainties in event-reconstruction efficiency calculation due to differences in particle-identification rates in data and simulation.

The first type of systematic uncertainty is estimated empirically to be $0.48 \%$ for kaon identification and $0.52 \%$ for pion identification. In order to estimate the second type of uncertainty, we proceed in two steps. First, we obtain particle-identification efficiency distribution for real data and simulated events using the standard BABAR calibration sample $\left(D^{* \pm} \rightarrow D_{K^{\mp} \pi^{ \pm}} \pi^{ \pm}\right)$but with stricter track selection criteria, as used in the present analysis. Then we plot their ratio, $\epsilon_{\text {data }} / \epsilon_{\mathrm{MC}}$, for both kaon and pion tracks. A comparison of this ratio for our (stricter) track selection criteria with the standatd track selection is shown in Fig 3.13. We repeat the whole analysis by applying this new correction to the signal reconstruction efficiency and take the difference in the final result as a systematic uncertainty. With the new data-simulation correction factor, we find that the average inverse efficiency changes by $-0.31 \%$ for $K^{-} \pi^{+} \pi^{0}$, by $0.58 \%$ for $\pi^{-} \pi^{+} \pi^{0}$, and by $-0.84 \%$ for $K^{-} K^{+} \pi^{0}$ modes. We apply this correction factor to the average weight and assign half the difference to systematics.

Combining the two types of uncertainties described above, we assign a systematic of $0.77 \%$ due to particle-identification to $\frac{\mathcal{B}\left(D^{0} \rightarrow \pi^{-} \pi^{+} \pi^{0}\right)}{\mathcal{B}\left(D^{0} \rightarrow K^{-} \pi^{+} \pi^{0}\right)}$ and $0.84 \%$ to $\frac{\mathcal{B}\left(D^{0} \rightarrow K^{-} K^{+} \pi^{0}\right)}{\mathcal{B}\left(D^{0} \rightarrow K^{-} \pi^{+} \pi^{0}\right)}$.

\subsection{Tracking efficiency systematics}

There are two types of issues related to data-simulation tracking efficiency correction and systematics :

- How well does simulation mimic the real data with respect to hit-pattern and resolution in the drift chamber and silicon tracker?

- As tracks traverse the detector they interact, scattering elastically or inelastically. The simulation has cross-sections built into it according to some hypothesis. How much does this cross-section differ from the one in real data? 

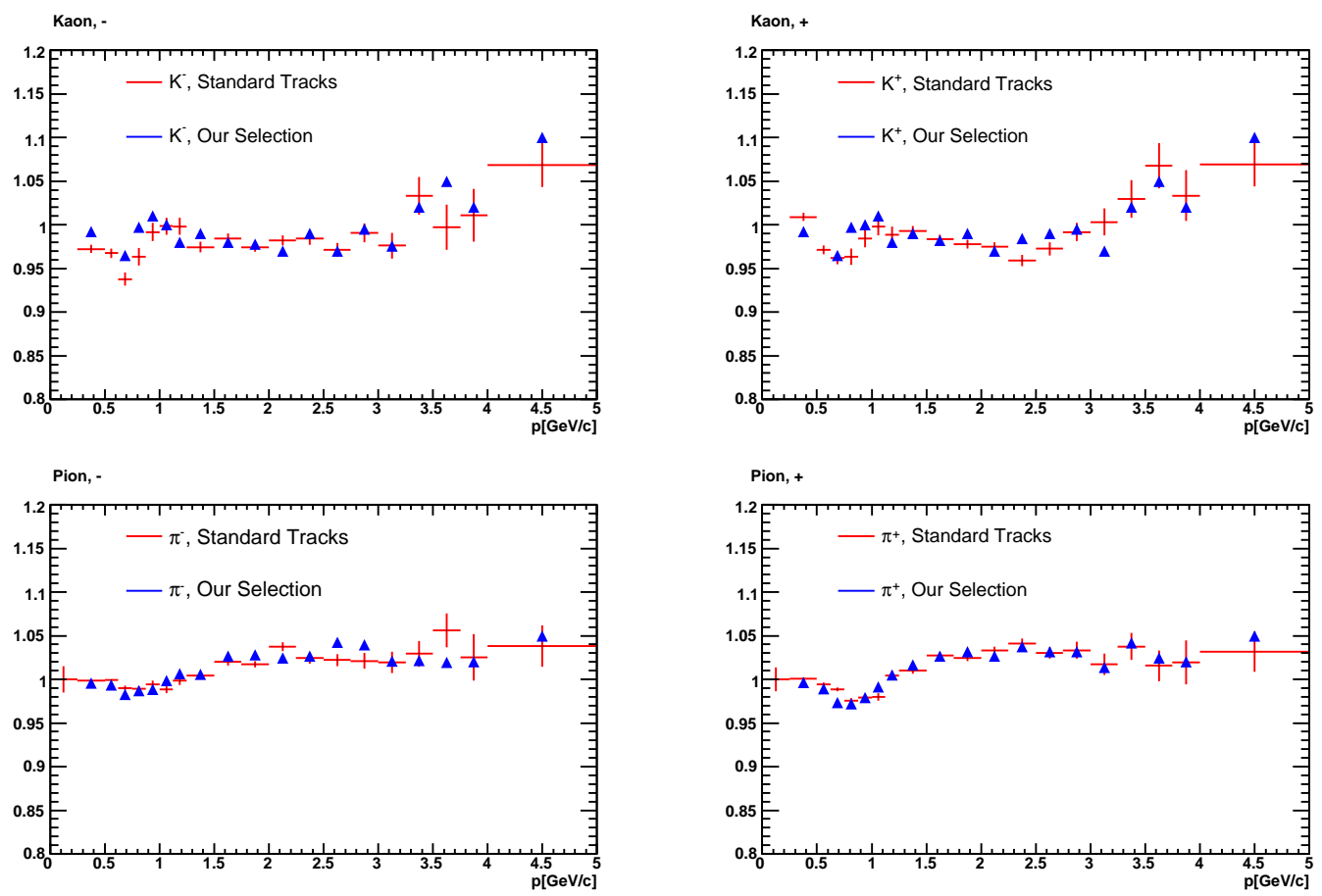

Figure 3.13: Comparison of the ratio of particle-identification rates in data and simulation for our track selection with those from the standard calibration.

The correction for the data-simulation difference due to the first effect is exactly the same for both kaons and pions and cancels out in the ratio of branching ratios. The correction for the second effect is also the same for kaons and pions but the uncertainty in this correction is slightly different for the two particles $(0.8 \pm 0.5 \%$ for kaons and $0.8 \pm 0.3 \%$ for pions) according to empirical studies. Accordingly, we conservatively assign a systematic uncertainty of $0.6 \%$ to both $\frac{\mathcal{B}\left(D^{0} \rightarrow \pi^{-} \pi^{+} \pi^{0}\right)}{\mathcal{B}\left(D^{0} \rightarrow K^{-} \pi^{+} \pi^{0}\right)}$ and $\frac{\mathcal{B}\left(D^{0} \rightarrow K^{-} K^{+} \pi^{0}\right)}{\mathcal{B}\left(D^{0} \rightarrow K^{-} \pi^{+} \pi^{0}\right)}$ ratios due to uncertainty in tracking efficiency corrections.

\subsection{Removal of $D^{0} \rightarrow K_{S}^{0} \pi^{0}$ from $D^{0} \rightarrow \pi^{-} \pi^{+} \pi^{0}$ events}

The $D^{0} \rightarrow K_{S}^{0} \pi^{0}$ decay is a Cabibbo favored decay and is a background for the $D^{0} \rightarrow \pi^{-} \pi^{+} \pi^{0}$ mode reconstruction. The long lifetime of the $K_{S}^{0}$ means that the two-body decay $D^{0} \rightarrow K_{S}^{0} \pi^{0}$ will not interfere with any other resonance and will form a narrow peak in the $\pi^{-} \pi^{+}$invariant mass plot. We estimate the level of $K_{S}^{0}$ contamination in $\pi^{-} \pi^{+} \pi^{0}$ sample by fitting the $m\left(\pi^{-} \pi^{+}\right)$distribution in this narrow peak region for the signal region of $\pi^{-} \pi^{+} \pi^{0}$ invariant mass. We get 538 $\pm 41 K_{S}^{0} \pi^{0}$ events and subtract this contribution from $\pi^{-} \pi^{+} \pi^{0}$ signal yield (and include the uncertainty in the number of $K_{S}^{0} \pi^{0}$ events in the final systematics). Figure 3.14 shows the fit for the invariant mass and flight-length distributions for the reconstructed $K_{S}^{0}$ candidates. 

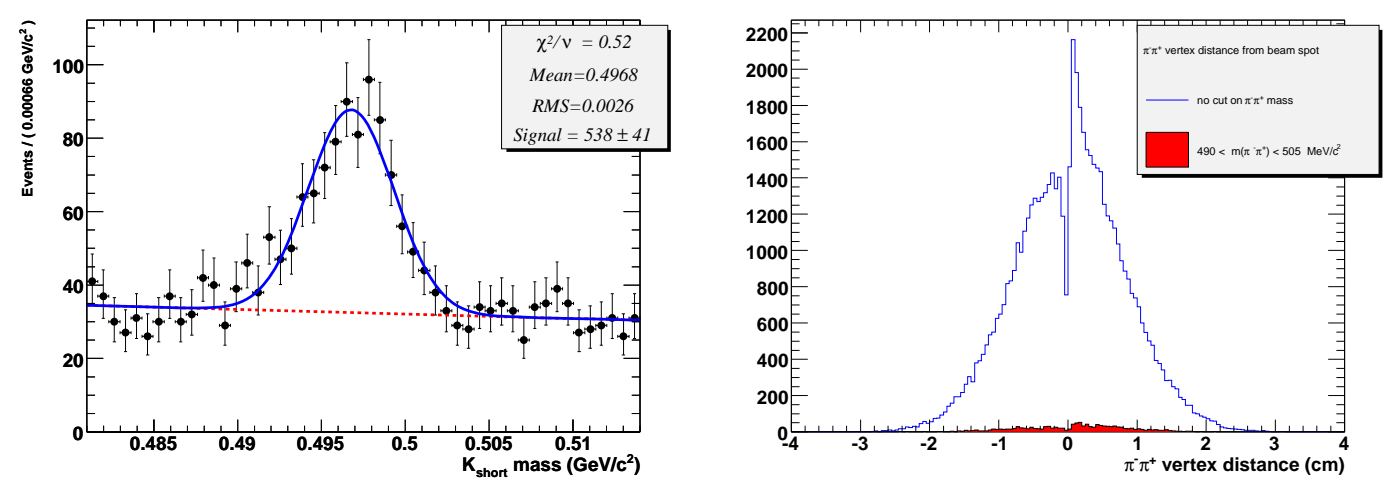

Figure 3.14: Fit for $K_{S}^{0}$ peak in the $\pi^{-} \pi^{+}$invariant mass plot (top) and the distribution of signed distance of the $\pi^{-} \pi^{+}$vertex from the beam spot (bottom).

\subsection{Summary of corrections}

Table 3.14 lists all the corrections applied to the ratio of branching ratios. The overall correction is $-0.47 \%$ for $\frac{\mathcal{B}\left(D^{0} \rightarrow \pi^{-} \pi^{+} \pi^{0}\right)}{\mathcal{B}\left(D^{0} \rightarrow K^{-} \pi^{+} \pi^{0}\right)}$ and $-0.49 \%$ for $\frac{\mathcal{B}\left(D^{0} \rightarrow K^{-} K^{+} \pi^{0}\right)}{\mathcal{B}\left(D^{0} \rightarrow K^{-} \pi^{+} \pi^{0}\right)}$.

\begin{tabular}{|c|c|c|}
\hline Correction & $\frac{\mathcal{B}\left(D^{0} \rightarrow \pi^{-} \pi^{+} \pi^{0}\right)}{\mathcal{B}\left(D^{0} \rightarrow K^{-} \pi^{+} \pi^{0}\right)}$ & $\frac{\mathcal{B}\left(D^{0} \rightarrow K^{-} K^{+} \pi^{0}\right)}{\mathcal{B}\left(D^{0} \rightarrow K^{-} \pi^{+} \pi^{0}\right)}$ \\
\hline \hline$P^{*}$ distribution (data/simulation) & $-0.48 \%$ & $+0.04 \%$ \\
\hline particle-identification & $+0.89 \%$ & $-0.53 \%$ \\
\hline$K_{S}^{0}$ Removal & $-0.88 \%$ & $0.0 \%$ \\
\hline Total & $-0.47 \%$ & $-0.49 \%$ \\
\hline
\end{tabular}

Table 3.5: Summary of corrections

\subsection{Summary of systematic uncertainties}

Table 3.6 lists results of all the systematic studies done for this analysis. Combining all these uncertainties, the final systematic error in $\frac{\mathcal{B}\left(D^{0} \rightarrow \pi^{-} \pi^{+} \pi^{0}\right)}{\mathcal{B}\left(D^{0} \rightarrow K^{-} \pi^{+} \pi^{0}\right)}$ measurement is $1.25 \%$ and in $\frac{\mathcal{B}\left(D^{0} \rightarrow K^{-} K^{+} \pi^{0}\right)}{\mathcal{B}\left(D^{0} \rightarrow K^{-} \pi^{+} \pi^{0}\right)}$ measurement is $1.71 \%$.

\subsection{Consistency check: analysis on disjoint data sub- samples}

For consistency check, we split the whole data sample into 10 disjoint sub-samples as described below, and analyze each sub-sample as an independent dataset. We do this to look for systematic variations as a function of charge or momentum outside the levels accounted for in our estimates of statistical and systematic uncertainties. To the extent that the results from disjoint samples agree within errors, we gain confidence that our error estimates for the combined, final sample are correct. First, 


\begin{tabular}{|c|c|c|}
\hline Uncertainty & $\frac{\mathcal{B}\left(D^{0} \rightarrow \pi^{-} \pi^{+} \pi^{0}\right)}{\mathcal{B}\left(D^{0} \rightarrow K^{-} \pi^{+} \pi^{0}\right)}$ & $\frac{\mathcal{B}\left(D^{0} \rightarrow K^{-} K^{+} \pi^{0}\right)}{\mathcal{B}\left(D^{0} \rightarrow K^{-} \pi^{+} \pi^{0}\right)}$ \\
\hline \hline Simulation statistics & $0.27 \%$ & $0.47 \%$ \\
\hline$\Delta m$ cut & $0.30 \%$ & $0.90 \%$ \\
\hline Background Subtraction & $0.60 \%$ & $0.90 \%$ \\
\hline Background PDF model & $0.16 \%$ & $0.13 \%$ \\
\hline Different $P^{*}$ in data and simulation & $0.24 \%$ & $0.02 \%$ \\
\hline Particle Identification & $0.77 \%$ & $0.84 \%$ \\
\hline Tracking Efficiency & $0.60 \%$ & $0.60 \%$ \\
\hline$K_{S}^{0}$ Removal & $0.07 \%$ & $0.0 \%$ \\
\hline Total & $1.25 \%$ & $1.71 \%$ \\
\hline
\end{tabular}

Table 3.6: Summary of systematic uncertainties

the data is divided into disjoint $D^{0}$ and $\bar{D}^{0}$ subsets. Then, each of these is divided into five ranges of laboratory momentum, each momentum range having a roughly equal number of events. They are as follows: $0<P_{l}<2.80 \mathrm{GeV} / c, 2.80<P_{l}<3.40$ $\mathrm{GeV} / c, 3.40<P_{l}<4.00 \mathrm{GeV} / c, 4.00<P_{l}<4.65 \mathrm{GeV} / c$, and $P_{l}>4.65 \mathrm{GeV} / c$. Table 3.7 summarizes the results for the disjoint samples. These are consistent with the main analysis results. Figure 3.16 shows the measured ratio of branching ratios for the disjoint data samples for the two Cabibbo-suppressed decay modes.
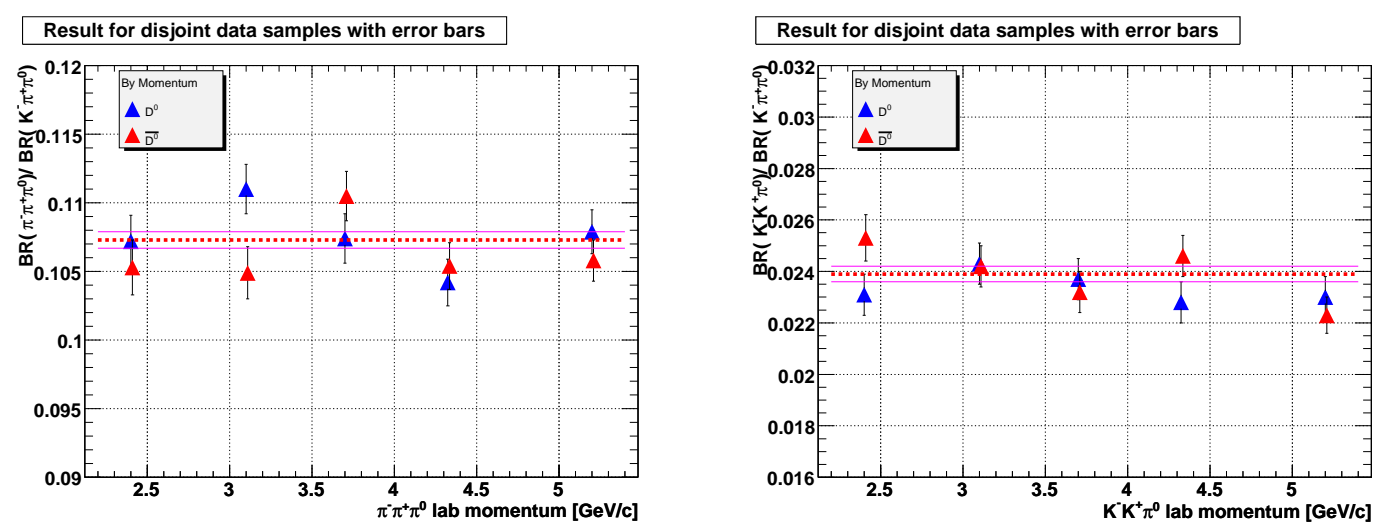

Figure 3.15: Measured $\frac{\mathcal{B}\left(D^{0} \rightarrow \pi^{-} \pi^{+} \pi^{0}\right)}{\mathcal{B}\left(D^{0} \rightarrow K^{-} \pi^{+} \pi^{0}\right)}$ (left) and $\frac{\mathcal{B}\left(D^{0} \rightarrow K^{-} K^{+} \pi^{0}\right)}{\mathcal{B}\left(D^{0} \rightarrow K^{-} \pi^{+} \pi^{0}\right)}$ (right) of disjoint data samples. The horizontal red line shows the result for the full data sample. Errors are statistical only.

\subsection{Results and conclusions}

Using Eqns 3.1 and 3.2, along with the results reported in Tables 3.3, 3.14 and 3.6, we obtain: $\frac{\mathcal{B}\left(D^{0} \rightarrow \pi^{-} \pi^{+} \pi^{0}\right)}{\mathcal{B}\left(D^{0} \rightarrow K^{-} \pi^{+} \pi^{0}\right)}=10.59 \pm 0.06$ (stat) \pm 0.13 (syst) $\times 10^{-2}$ and $\frac{\mathcal{B}\left(D^{0} \rightarrow K^{-} K^{+} \pi^{0}\right)}{\mathcal{B}\left(D^{0} \rightarrow K^{-} \pi^{+} \pi^{0}\right)}=$ $2.37 \pm 0.03$ (stat) \pm 0.04 (syst) $\times 10^{-2}$. 


\begin{tabular}{|c|c|c|c|c|}
\hline Data & Mode & Signal & ave. weight & av. weight from fit \\
\hline \multirow[t]{3}{*}{$\bar{D}^{\sigma}$} & $\pi^{-} \pi^{+} \pi^{0}$ & $30976 \pm 239$ & $9.2603 \pm 0.0287$ & $9.3966 \pm 0.0298$ \\
\hline & $K^{-} \pi^{+} \pi^{0}$ & $253781 \pm 529$ & $10.7681 \pm 0.0263$ & $10.7365 \pm 0.0282$ \\
\hline & $K^{-} K^{+} \pi^{0}$ & $5420 \pm 83$ & $12.3497 \pm 0.0723$ & $12.1916 \pm 0.0822$ \\
\hline \multirow[t]{3}{*}{$\bar{D}^{0}$} & $\pi^{-} \pi^{+} \pi^{0}$ & $30413 \pm 215$ & $9.5853 \pm 0.0279$ & $9.7048 \pm 0.0281$ \\
\hline & $K^{-} \pi^{+} \pi^{0}$ & $252138 \pm 538$ & $10.7251 \pm 0.0258$ & $10.6985 \pm 0.0206$ \\
\hline & $K^{-} K^{+} \pi^{0}$ & $5359 \pm 83$ & $12.8629 \pm 0.0756$ & $12.6800 \pm 0.0763$ \\
\hline \multirow{3}{*}{$\begin{array}{l}D^{0} \text { 1st Mom. Bin } \\
\left(0<P_{l}<2.80 \mathrm{GeV} / c\right)\end{array}$} & $\pi^{-} \pi^{+} \pi^{0}$ & $5822 \pm 109$ & $12.0442 \pm 0.0667$ & $12.3367 \pm 0.0638$ \\
\hline & $K^{-} \pi^{+} \pi^{0}$ & $46382 \pm 232$ & $14.9154 \pm 0.0572$ & $14.8734 \pm 0.0540$ \\
\hline & $K^{-} K^{+} \pi^{0}$ & $1010 \pm 35$ & $17.4724 \pm 0.1709$ & $17.9814 \pm 0.1796$ \\
\hline \multirow{3}{*}{$\begin{array}{l}\overline{\bar{D}}^{0} \text { 1st Mom. Bin } \\
\left(0<P_{l}<2.80 \mathrm{GeV} / c\right)\end{array}$} & $\pi^{-} \pi^{+} \pi^{0}$ & $5698 \pm 101$ & $13.3347 \pm 0.0689$ & $13.7304 \pm 0.0657$ \\
\hline & $K^{-} \pi^{+} \pi^{0}$ & $45915 \pm 241$ & $15.8238 \pm 0.0599$ & $15.6749 \pm 0.0515$ \\
\hline & $K^{-} K^{+} \pi^{0}$ & $955 \pm 35$ & $19.5717 \pm 0.1645$ & $19.6980 \pm 0.1809$ \\
\hline \multirow{3}{*}{$\begin{array}{l}D^{0} \text { 2nd Mom. Bin } \\
\left(2.80<P_{l}<3.40 \mathrm{GeV} / c\right)\end{array}$} & $\pi^{-} \pi^{+} \pi^{0}$ & $5891 \pm 98$ & $5.5272 \pm 0.0672$ & $5.6689 \pm 0.0659$ \\
\hline & $K^{-} \pi^{+} \pi^{0}$ & $50964 \pm 236$ & $6.2629 \pm 0.0611$ & $6.3026 \pm 0.0541$ \\
\hline & $K^{-} K^{+} \pi^{0}$ & $1097 \pm 36$ & $7.6498 \pm 0.1651$ & $7.7257 \pm 0.1880$ \\
\hline \multirow{3}{*}{$\begin{array}{l}\bar{D}^{0} \text { 2nd Mom. Bin } \\
\left(2.80<P_{l}<3.40 \mathrm{GeV} / c\right)\end{array}$} & $\pi^{-} \pi^{+} \pi^{0}$ & $5937 \pm 108$ & $5.6762 \pm 0.0649$ & $5.8176 \pm 0.0692$ \\
\hline & $K^{-} \pi^{+} \pi^{0}$ & $50698 \pm 241$ & $6.1144 \pm 0.0570$ & $6.0922 \pm 0.0610$ \\
\hline & $K^{-} K^{+} \pi^{0}$ & $1035 \pm 35$ & $7.6226 \pm 0.1687$ & $7.8588 \pm 0.1917$ \\
\hline \multirow{3}{*}{$\begin{array}{l}D^{0} \text { 3rd Mom. Bin } \\
\left(3.40<P_{l}<4.00 \mathrm{GeV} / c\right)\end{array}$} & $\pi^{-} \pi^{+} \pi^{0}$ & $5923 \pm 91$ & $5.2437 \pm 0.0644$ & $5.3373 \pm 0.0657$ \\
\hline & $K^{-} \pi^{+} \pi^{0}$ & $51135 \pm 238$ & $5.9546 \pm 0.0563$ & $5.9651 \pm 0.0672$ \\
\hline & $K^{-} K^{+} \pi^{0}$ & $1141 \pm 37$ & $6.6878 \pm 0.1720$ & $6.6591 \pm 0.1612$ \\
\hline \multirow{3}{*}{$\begin{array}{l}\bar{D}^{0} \text { 3rd Mom. Bin } \\
\left(3.40<P_{l}<4.00 \mathrm{GeV} / c\right)\end{array}$} & $\pi^{-} \pi^{+} \pi^{0}$ & $5967 \pm 94$ & $5.3260 \pm 0.0675$ & $5.4245 \pm 0.0635$ \\
\hline & $K^{-} \pi^{+} \pi^{0}$ & $50629 \pm 228$ & $5.7211 \pm 0.0506$ & $5.7522 \pm 0.0619$ \\
\hline & $K^{-} K^{+} \pi^{0}$ & $1091 \pm 38$ & $6.7398 \pm 0.1642$ & $6.7785 \pm 0.1964$ \\
\hline \multirow{3}{*}{$\begin{array}{l}D^{0} \text { 4th Mom. Bin } \\
\left(4.00<P_{l}<4.65 \mathrm{GeV} / c\right)\end{array}$} & $\pi^{-} \pi^{+} \pi^{0}$ & $5935 \pm 111$ & $6.3010 \pm 0.0628$ & $6.3262 \pm 0.0683$ \\
\hline & $K^{-} \pi^{+} \pi^{0}$ & $50209 \pm 231$ & $7.1674 \pm 0.0591$ & $7.1662 \pm 0.0576$ \\
\hline & $K^{-} K^{+} \pi^{0}$ & $1082 \pm 38$ & $8.1019 \pm 0.1926$ & $8.2742 \pm 0.1707$ \\
\hline \multirow{3}{*}{$\begin{array}{l}\bar{D}^{0} 4 \text { th Mom. Bin } \\
\left(4.00<P_{l}<4.65 \mathrm{GeV} / c\right)\end{array}$} & $\pi^{-} \pi^{+} \pi^{0}$ & $5859 \pm 88$ & $6.3084 \pm 0.0613$ & $6.3143 \pm 0.0622$ \\
\hline & $K^{-} \pi^{+} \pi^{0}$ & $50518 \pm 239$ & $7.1590 \pm 0.0535$ & $7.1509 \pm 0.0582$ \\
\hline & $K^{-} K^{+} \pi^{0}$ & $1139 \pm 36$ & $8.3149 \pm 0.1795$ & $8.2573 \pm 0.1902$ \\
\hline \multirow{3}{*}{$\begin{array}{l}D^{0} \text { 5th Mom. Bin } \\
\left(P_{l}>4.65 \mathrm{GeV} / c\right)\end{array}$} & $\pi^{-} \pi^{+} \pi^{0}$ & $6834 \pm 93$ & $16.0507 \pm 0.0544$ & $16.1518 \pm 0.0562$ \\
\hline & $K^{-} \pi^{+} \pi^{0}$ & $54672 \pm 243$ & $18.6637 \pm 0.0494$ & $18.3631 \pm 0.0504$ \\
\hline & $K^{-} K^{+} \pi^{0}$ & $1041 \pm 43$ & $21.1034 \pm 0.1602$ & $21.6436 \pm 0.1711$ \\
\hline \multirow{3}{*}{$\begin{array}{l}\bar{D}^{0} \text { 5th Mom. Bin } \\
\left(P_{l}>4.65 \mathrm{GeV} / c\right)\end{array}$} & $\pi^{-} \pi^{+} \pi^{0}$ & $6919 \pm 98$ & $16.2426 \pm 0.0556$ & $16.5615 \pm 0.0565$ \\
\hline & $K^{-} \pi^{+} \pi^{0}$ & $55046 \pm 249$ & $18.8196 \pm 0.0507$ & $18.7154 \pm 0.0499$ \\
\hline & $K^{-} K^{+} \pi^{0}$ & $1175 \pm 35$ & $21.3309 \pm 0.1621$ & $21.7394 \pm 0.1668$ \\
\hline
\end{tabular}

Table 3.7: Measurements of disjoint samples. 
Using the PDG [20] fit value $(13.9 \pm 0.9 \%)$ of the branching ratio of $D^{0} \rightarrow$ $K^{-} \pi^{+} \pi^{0}$, the absolute branching ratios for the two decay modes are: $\mathcal{B}\left(D^{0} \rightarrow\right.$ $\left.\pi^{-} \pi^{+} \pi^{0}\right)=(1.472 \pm 0.008 \pm 0.018 \pm 0.097) \times 10^{-2}$ and $\mathcal{B}\left(D^{0} \rightarrow K^{-} K^{+} \pi^{0}\right)=$ $(0.332 \pm 0.004 \pm 0.006 \pm 0.021) \times 10^{-2}$, where the first error is statistical, the second error is systematic, and the third error is due to uncertainty in $\mathcal{B}\left(D^{0} \rightarrow K^{-} \pi^{+} \pi^{0}\right)$. Table 3.8 compares the previous measurements with those from this analysis.

\begin{tabular}{|c|c|c|}
\hline Ratios of Branching ratios & this analysis & PDG Value \\
\hline \hline$\frac{\mathcal{B}\left(D^{0} \rightarrow \pi^{-} \pi^{+} \pi^{0}\right)}{\mathcal{B}\left(D^{0} \rightarrow K^{-} \pi^{+} \pi^{0}\right)}$ & $10.59 \pm 0.06 \pm 0.13 \%$ & $8.40 \pm 3.11 \%$ \\
\hline$\frac{\mathcal{B}\left(D^{0} \rightarrow K^{-} K^{+} \pi^{0}\right)}{\mathcal{B}\left(D^{0} \rightarrow K^{-} \pi^{+} \pi^{0}\right)}$ & $2.37 \pm 0.03 \pm 0.04 \%$ & $0.92 \pm 0.31 \%$ \\
\hline
\end{tabular}

Table 3.8: A comparison of the previous PDG [20] results with this analysis.

The decay rates for each of the processes considered in this analysis are proportional to the product of the relevant quantum mechanical matrix element and phase space factor. For each process we can write

$$
\Gamma=\left\langle|\mathcal{M}|^{2}\right\rangle \times \Phi
$$

where $\Gamma$ is the decay rate to a particular three-body decay integrated over the Dalitz plot, $\left\langle|\mathcal{M}|^{2}\right\rangle$ is the average value of $|\mathcal{M}|^{2}$ over the Dalitz plot, and $\Phi$ is the three-body phase space which is proportional to the area of the Dalitz plot. Because the mass of the kaon is greater than the mass of the pion, the phase space available for the decay $D^{0} \rightarrow K^{-} K^{+} \pi^{0}$ is less than that for $D^{0} \rightarrow K^{-} \pi^{+} \pi^{0}$, which is less than that for $D^{0} \rightarrow \pi^{-} \pi^{+} \pi^{0}$. This can be seen in Fig. 3.16 which shows the boundaries of the the three Dalitz plots. Table 3.9 summarizes the areas, calculated numerically. The ratios of the matrix elements are calculated by dividing the ratios of the decay rates (branching ratios) by the ratios of the Dalitz plot areas. Combining the statistical and systematic errors, we find:

$$
\begin{aligned}
& \frac{|\mathcal{M}|^{2}\left(D^{0} \rightarrow \pi^{-} \pi^{+} \pi^{0}\right)}{|\mathcal{M}|^{2}\left(D^{0} \rightarrow K^{-} \pi^{+} \pi^{0}\right)}=0.0668 \pm 0.0004 \pm 0.0008, \\
& \frac{|\mathcal{M}|^{2}\left(D^{0} \rightarrow K^{-} K^{+} \pi^{0}\right)}{|\mathcal{M}|^{2}\left(D^{0} \rightarrow K^{-} \pi^{+} \pi^{0}\right)}=0.0453 \pm 0.0006 \pm 0.0008, \\
& \frac{|\mathcal{M}|^{2}\left(D^{0} \rightarrow K^{-} K^{+} \pi^{0}\right)}{|\mathcal{M}|^{2}\left(D^{0} \rightarrow \pi^{-} \pi^{+} \pi^{0}\right)}=0.6781 \pm 0.0007 \pm 0.0011 \text {. }
\end{aligned}
$$

To the extent that the differences in the matrix elements are only due to Cabibbosuppression at the quark level, as seen in Fig. 1.4, the ratios of the matrix elements squared for singly Cabibbo-suppressed decays to that for the Cabibbo-favored decay should be approximately $\sin ^{2} \theta_{C} \approx 0.05$ and ratio of the matrix elements squared for the two singly Cabibbo-suppressed decays should be the unity. We can compare these results to those for the two-body decays where the phase space is proportional to the magnitude of the decay momentum in the $D^{0}$ center-of-mass frame. For these we use the PDG averages to calculate

$$
\frac{|\mathcal{M}|^{2}\left(D^{0} \rightarrow \pi^{-} \pi^{+}\right)}{|\mathcal{M}|^{2}\left(D^{0} \rightarrow K^{-} \pi^{+}\right)}=0.0338 \pm 0.0009
$$




$$
\begin{aligned}
& \frac{|\mathcal{M}|^{2}\left(D^{0} \rightarrow K^{-} K^{+}\right)}{|\mathcal{M}|^{2}\left(D^{0} \rightarrow K^{-} \pi^{+}\right)}=0.1114 \pm 0.0023 \\
& \frac{|\mathcal{M}|^{2}\left(D^{0} \rightarrow K^{-} K^{+}\right)}{|\mathcal{M}|^{2}\left(D^{0} \rightarrow \pi^{-} \pi^{+}\right)}=3.5289 \pm 0.0026
\end{aligned}
$$

In summary, we have measured the ratios of branching ratios for the three-body decays $D^{0} \rightarrow \pi^{-} \pi^{+} \pi^{0}, D^{0} \rightarrow K^{-} K^{+} \pi^{0}$, and $D^{0} \rightarrow K^{-} \pi^{+} \pi^{0}$. The ratios of the singly Cabibbo-suppressed decay rates relative to the Cabibbo-favored decay rate are measured with significantly better precision than previously reported. The average matrix elements squared for both the singly Cabibbo-suppressed decays studied here, $D^{0} \rightarrow \pi^{-} \pi^{+} \pi^{0}$ and $D^{0} \rightarrow K^{-} K^{+} \pi^{0}$, are roughly a factor of $\sin ^{2} \theta_{C}$ smaller than that for the corresponding Cabibbo-favored decay $D^{0} \rightarrow K^{-} \pi^{+} \pi^{0}$. This can be compared to the case for the corresponding two-body decays where the matrix elements squared for the $D^{0} \rightarrow K^{-} K^{+}$and $D^{0} \rightarrow \pi^{-} \pi^{+}$decays differ roughly by a factor of two.

\begin{tabular}{|c|c|}
\hline Mode & area \\
\hline \hline$\pi^{-} \pi^{+} \pi^{0}$ & 5.053 \\
$K^{-} \pi^{+} \pi^{0}$ & 3.188 \\
$K^{-} K^{+} \pi^{0}$ & 1.668 \\
\hline
\end{tabular}

Table 3.9: Areas covered by Dalitz plots.

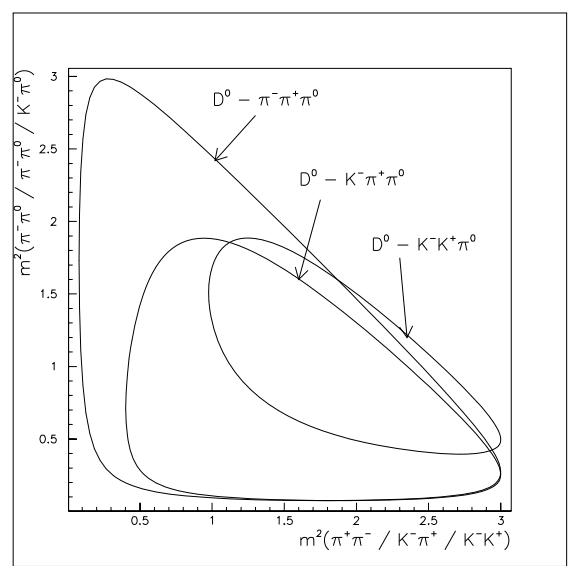

Figure 3.16: Dalitz boundaries 


\section{Chapter 4}

\section{Amplitude Analysis of the Decay $D^{0} \rightarrow K^{-} K^{+} \pi^{0}$}

Using $385 \mathrm{fb}^{-1}$ of $e^{+} e^{-}$collisions, we study the amplitudes of the singly Cabibbosuppressed decay $D^{0} \rightarrow K^{-} K^{+} \pi^{0}[21]$. We measure the strong phase difference between the $\bar{D}^{0}$ and $D^{0}$ decays to $K^{*}(892)^{+} K^{-}$to be $-35.5^{\circ} \pm 1.9^{\circ}$ (stat) $\pm 2.2^{\circ}$ (syst), and their amplitude ratio to be $0.599 \pm 0.013$ (stat) \pm 0.011 (syst). We observe contributions from the $K \pi$ and $K^{-} K^{+}$scalar and vector amplitudes, and analyze their angular moments. We find no evidence for charged $\kappa$, nor for higher spin states. We also perform a partial-wave analysis of the $K^{-} K^{+}$system in a limited mass range.

\subsection{Introduction}

The amplitudes describing $D$ meson weak decays into three-body final states are dominated by intermediate resonances that lead to highly nonuniform intensity distributions in the available phase space. Analyses of these distributions have led to new insights into the role of the light-meson systems produced [22]. The $K^{ \pm} \pi^{0}$ systems from the decay $D^{0} \rightarrow K^{-} K^{+} \pi^{0}{ }^{1}$ can provide information on the $K \pi S$-wave (spin-0) amplitude in the mass range 0.6-1.4 GeV/c $c^{2}$, and hence on the possible existence of the $\kappa(800)$, reported to date only in the neutral state $\left(\kappa^{0} \rightarrow K^{-} \pi^{+}\right)$[23]. If the $\kappa$ has isospin $1 / 2$, it should be observable also in the charged states. Results of the present analysis can be an input for extracting the $C P$-violating phase $\gamma=\arg \left(-V_{u d} V_{u b}^{*} / V_{c d} V_{c b}^{*}\right)$ of the quark mixing matrix by exploiting interference structure in the Dalitz plot [19] from the decay $B^{ \pm} \rightarrow D_{K^{-} K^{+} \pi^{0}}^{0} K^{ \pm}[24,25]$. Singly Cabibbo-suppressed decays are also important because they might be sensitive to direct $C P$ violation in charm decays [9], the discovery of which might indicate physics beyond the Standard Model.

\footnotetext{
${ }^{1}$ Reference to the charge-conjugate decay is implied throughout this chapter unless stated otherwise. The initial state referred to is $D^{0}$, not $\bar{D}^{0}$.
} 


\subsection{Event selection}

We perform the present analysis on $385 \mathrm{fb}^{-1}$ of $e^{+} e^{-}$collision data collected at and around $10.58 \mathrm{GeV}$ center-of-mass (CM) energy with the BABAR detector [12] at the PEP-II storage ring. We distinguish $D^{0}$ from $\bar{D}^{0}$ by reconstructing the decays $D^{*+} \rightarrow D^{0} \pi^{+}$and $D^{*-} \rightarrow \bar{D}^{0} \pi^{-}$. The event-selection criteria are the same as those used in our measurement of the branching ratio of the decay $D^{0} \rightarrow K^{-} K^{+} \pi^{0}$ [18]. In particular, we require that the $\mathrm{CM}$ momentum of $D^{0}$ candidate be greater than $2.77 \mathrm{GeV} / c$, and that $\left|m_{D^{*+}}-m_{D^{0}}-145.4\right|<0.6 \mathrm{MeV} / c^{2}$, where $m$ refers to a reconstructed invariant mass. To minimize uncertainty from background shape, we choose a sample of very high purity $(\sim 98.1 \%)$ using $1855<m_{D^{0}}<1875 \mathrm{MeV} / c^{2}$, and find $11278 \pm 110$ signal events.

\subsection{Efficiency calculation}

We estimate the signal efficiency for each event as a function of its position in the Dalitz plot using simulated $D^{0} \rightarrow K^{-} K^{+} \pi^{0}$ decays from $e^{+} e^{-} \rightarrow c \bar{c}$ events, generated uniformly in the available phase space. To correct for differences in particleidentification rates in data and simulation, we determine the ratio of these for each track, and apply an event-by-event correction factor.

To obtain the efficiency function, a simulation sample of about 1.5 million $D^{0} \rightarrow$ $K^{-} K^{+} \pi^{0}$ signal events uniformly populated in phase space was generated. A track parameters-dependent particle-identification correction was applied to this sample on an event-by-event basis, using the ratio of signal reconstruction efficiencies in data and simulation in bins of momentum and polar angle. For this purpose, the generated and reconstructed Dalitz plots are divided into $\mathrm{N} \times \mathrm{N}$ cells, $\mathrm{N}$ chosen in such a way to have at least 25 reconstructed events per cell on average. The efficiency map is then obtained by dividing the reconstructed Dalitz plot over the generated one. The efficiency is parametrized as a 3rd order polynomial in the two Dalitz plot variables $s_{+0}\left(=m_{K^{+} \pi^{0}}^{2}\right)$ and $s_{-0}\left(=m_{K^{-} \pi^{0}}^{2}\right)$ as

$$
\begin{aligned}
\epsilon\left(s_{+0}, s_{-0}\right)=1 & +s_{1}\left(s_{+0}+s_{-0}\right) \\
& +s_{2}\left(s_{+0}^{2}+s_{-0}^{2}\right)+s_{3}\left(s_{+0}^{3}+s_{-0}^{3}\right) \\
& +s_{4}\left(s_{-0} s_{+0}^{2}+s_{+0} s_{-0}^{2}\right)+s_{5}\left(s_{+0} s_{-0}\right) .
\end{aligned}
$$

In addition, $\epsilon\left(s_{+0}, s_{-0}\right) \equiv 0$ for all points outside the physical boundary of the Dalitz plot. The results of the fit are

$s_{1}=-0.58 \pm 0.02$

$s_{2}=0.30 \pm 0.06$

$s_{3}=-0.15 \pm 0.05$

$s_{4}=0.27 \pm 0.11$

$s_{5}=0.39 \pm 0.12$ 


\subsection{Parametrization of amplitudes}

Neglecting $C P$ violation in $D$ meson decays, we define the $D^{0}\left(\bar{D}^{0}\right)$ decay amplitude $\mathcal{A}(\overline{\mathcal{A}})$ in the $D^{0} \rightarrow K^{-} K^{+} \pi^{0}$ Dalitz plot of Fig. 4.1, as:

$$
\begin{aligned}
& \mathcal{A}\left[D^{0} \rightarrow K^{-} K^{+} \pi^{0}\right] \equiv f_{D^{0}}\left(m_{K^{+} \pi^{0}}^{2}, m_{K^{-} \pi^{0}}^{2}\right), \\
& \overline{\mathcal{A}}\left[\bar{D}^{0} \rightarrow K^{+} K^{-} \pi^{0}\right] \equiv f_{D^{0}}\left(m_{K^{-} \pi^{0}}^{2}, m_{K^{+} \pi^{0}}^{2}\right) .
\end{aligned}
$$

The complex quantum mechanical amplitude $f$ is a coherent sum of all relevant quasitwo-body $D^{0} \rightarrow(r \rightarrow A B) C$ isobar model [26] resonances, $f=\sum_{r} a_{r} e^{i \phi_{r}} A_{r}(s)$. Here $s=m_{A B}^{2}$, and $A_{r}$ is the resonance amplitude. We obtain coefficients $a_{r}$ and $\phi_{r}$ from a likelihood fit. The probability density function for signal events is $|f|^{2}$. We model incoherent background empirically using events from the lower sideband of the $m_{D^{0}}[18]$ distribution.
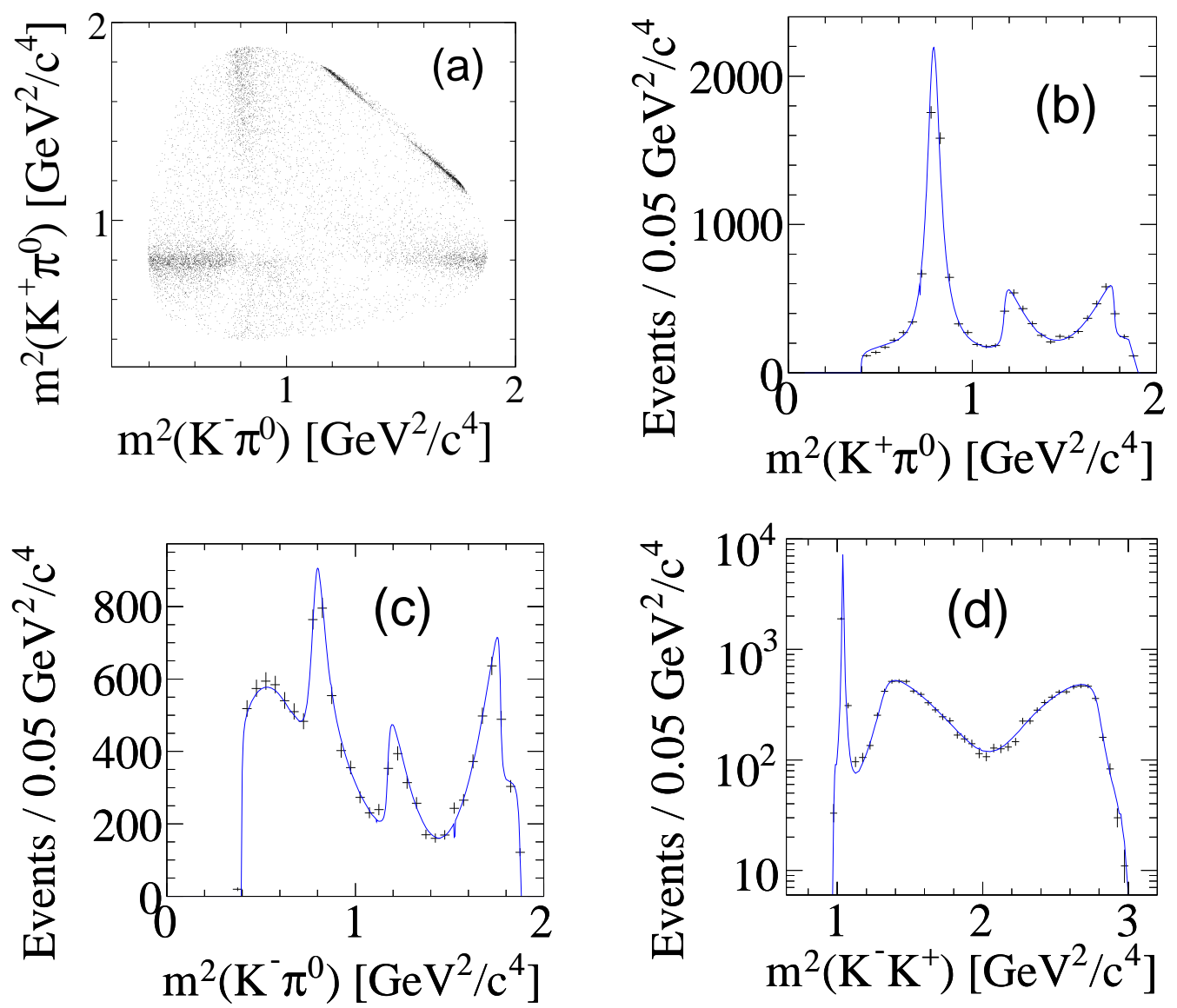

Figure 4.1: Dalitz plot for $D^{0} \rightarrow K^{-} K^{+} \pi^{0}$ data (a), and the corresponding squared invariant mass projections $(\mathrm{b}-\mathrm{d})$. The three-body invariant mass of the $D^{0}$ candidate is constrained to the nominal value. In plots $(b-d)$, the dots (with error bars, black) are data points and the solid lines (blue) correspond to the best isobar fit models. 


\subsection{Parametrization of $P$ - and $D$ - wave amplitudes}

For $D^{0}$ decays to spin-1 ( $P$-wave) and spin-2 states, we use the Breit-Wigner amplitude,

$$
\begin{aligned}
A_{B W}(s) & =\mathcal{M}_{L}(s, p) \frac{1}{M_{0}^{2}-s-i M_{0} \Gamma(s)}, \\
\Gamma(s) & =\Gamma_{0}\left(\frac{M_{0}}{\sqrt{s}}\right)\left(\frac{p}{p_{0}}\right)^{2 L+1}\left[\frac{\mathcal{F}_{L}(p)}{\mathcal{F}_{L}\left(p_{0}\right)}\right]^{2},
\end{aligned}
$$

where $M_{0}\left(\Gamma_{0}\right)$ is the resonance mass (width) [20], $L$ is the angular momentum quantum number, $p$ is the momentum of either daughter in the resonance rest frame, and $p_{0}$ is the value of $p$ when $\mathrm{s}=M_{0}^{2}$. The function $\mathcal{F}_{L}$ is the Blatt-Weisskopf barrier factor [27]: $\mathcal{F}_{0}=1, \mathcal{F}_{1}=1 / \sqrt{1+R p^{2}}$, and $\mathcal{F}_{2}=1 / \sqrt{9+3 R p^{2}+R p^{4}}$, where we take the meson radial parameter $R$ to be $1.5 \mathrm{GeV}^{-1}$ [28]. We define the spin part of the amplitude, $\mathcal{M}_{L}$, as: $\mathcal{M}_{0}=M_{D^{0}}^{2}, \mathcal{M}_{1}=-2 \overrightarrow{p_{A}} \cdot \overrightarrow{p_{C}}$, and $\mathcal{M}_{2}=\frac{4}{3}$ $\left.\left[3 \overrightarrow{p_{A}} \cdot \overrightarrow{p_{C}}\right)^{2}-\left|\overrightarrow{p_{A}}\right|^{2} \cdot\left|\overrightarrow{p_{C}}\right|^{2}\right] M_{D^{0}}^{-2}$, where $M_{D^{0}}$ is the nominal $D^{0}$ mass, and $\overrightarrow{p_{i}}$ is the 3 -momentum of particle $i$ in the resonance rest frame.

\subsection{Parametrization of $K^{ \pm} \pi^{0} S$-wave amplitudes}

For $D^{0}$ decays to $K^{ \pm} \pi^{0} S$-wave states, we consider three amplitude models. One model uses the LASS amplitude for $K^{-} \pi^{+} \rightarrow K^{-} \pi^{+}$elastic scattering [29],

$$
\begin{aligned}
A_{K \pi(S)}(s)= & \frac{\sqrt{s}}{p} \sin \delta(s) e^{i \delta(s)} \\
& \delta(s)=\cot ^{-1}\left(\frac{1}{p a}+\frac{b p}{2}\right)+\cot ^{-1}\left(\frac{M_{0}^{2}-s}{M_{0} \Gamma_{0} \cdot \frac{M_{0}}{\sqrt{s}} \cdot \frac{p}{p_{0}}}\right),
\end{aligned}
$$

where $M_{0}\left(\Gamma_{0}\right)$ refers to the $K_{0}^{*}(1430)$ mass (width), $a=1.95 \pm 0.09 \mathrm{GeV}^{-1} c$, and $b=1.76 \pm 0.36 \mathrm{GeV}^{-1} c$. The unitary nature of Eq. 4.6 provides a good description of the amplitude up to $1.45 \mathrm{GeV} / c^{2}$ (i.e., $K \eta^{\prime}$ threshold). In Eq. 4.7, the first term is a nonresonant contribution defined by a scattering length $a$ and an effective range $b$, and the second term represents the $K_{0}^{*}(1430)$ resonance. The phase space factor $\sqrt{s} / p$ converts the scattering amplitude, as measured by LASS, to the invariant amplitude required in the present analysis. Our second model uses the E-791 results for the $K^{-} \pi^{+} S$-wave amplitude from an energy-independent partial-wave analysis in the decay $D^{+} \rightarrow K^{-} \pi^{+} \pi^{+}$[30]. The third model uses a coherent sum of a uniform nonresonant term, and Breit-Wigner terms for the $\kappa(800)$ and $K_{0}^{*}(1430)$ resonances.

\subsection{Nature of $K^{ \pm} \pi^{0} S$-wave}

In Fig. 4.7 we compare the $K \pi S$-wave amplitude from the E-791 analysis [30] to the LASS amplitude of Eqs. 4.6-4.7. For easy comparison, we have normalized the LASS amplitude in Fig. 4.7a approximately to the E-791 measurements with $\sqrt{s}>1.15 \mathrm{GeV} / c^{2}$, and have reduced the LASS phase, $\delta(s)$, in Fig. $4.7 \mathrm{~b}$ by $80^{\circ}$. We 
then observe good agreements in the mass dependence of amplitude and phase for $\sqrt{s}>1.15 \mathrm{GeV} / c^{2}$. As the mass decreases from $1.15 \mathrm{GeV} / c^{2}$, the E-791 amplitude increases while the LASS amplitude decreases, with the ratio finally reaching $\sim 1.7$ at threshold. At the same time, their phase difference increases to $\sim 40^{\circ}$ at threshold. This behavior might be due to the form factor describing $D^{0}$ decay to a $K \pi S$ wave system and a bachelor $\bar{K}$. Since no centrifugal barrier is involved, such an effect should be more significant for $S$-wave than for higher spin waves because of the larger overlap between the initial and final state wave functions. However, the inverse momentum of the $K \pi$ system in the $D^{0}$ rest frame increases from 0.27 Fermi at $K \pi$ threshold to 0.48 Fermi at $1.15 \mathrm{GeV} / c^{2}$, therefore any form factor effect would decrease with increasing $K \pi$ mass. If the effect is essentially gone by $1.15 \mathrm{GeV} / c^{2}$, similar mass dependence of amplitude and phase in $D^{0}$ decay and $K \pi$ scattering would be observable at higher mass values, in agreement with Fig. 4.7. In the present analysis, we make an attempt to distinguish between the two rather different $K \pi$ $S$-wave mass dependences in the region below $\sim 1.15 \mathrm{GeV} / c^{2}$. In each case, we also allow the fit to determine the strength and phase of these amplitudes relative to the $K^{*}(892)^{+}$reference.

\subsection{Parametrization of $K^{-} K^{+} S$-wave amplitude}

We describe the $D^{0}$ decay to a $K^{-} K^{+} S$-wave state by a coupled-channel BreitWigner amplitude for the $f_{0}(980)$ and $a_{0}(980)$ resonances, with their respective couplings to $\pi \pi, K \bar{K}$ and $\eta \pi, K \bar{K}$ final states [31],

$$
A_{f_{0}\left[a_{0}\right]}(s)=\frac{M_{D^{0}}^{2}}{M_{0}^{2}-s-i\left(g_{1}^{2} \rho_{\pi \pi[\eta \pi]}+g_{2}^{2} \rho_{K \bar{K}}\right)} .
$$

Here $\rho$ represents Lorentz invariant phase space, $2 p / \sqrt{s}$. For the $f_{0}(980)$, we use the BES [32] parameter values $M_{0}=965 \pm 10 \mathrm{MeV} / c^{2}, g_{1}^{2}=165 \pm 18 \mathrm{MeV}^{2} / \mathrm{c}^{4}$, and $g_{2}^{2} / g_{1}^{2}=4.21 \pm 0.33$. For the $a_{0}(980)$, we use the Crystal Barrel [33] values $M_{0}=$ $999 \pm 2 \mathrm{MeV} / c^{2}, g_{1}=324 \pm 15 \mathrm{MeV} / c^{2}$, and $g_{1}^{2} / g_{2}^{2}=1.03 \pm 0.14$. Only the high mass tails of $f_{0}(980)$ and $a_{0}(980)$ are observable, as shown in Fig. 4.3a. They are similar, so we try a model for each as a description of the $K^{-} K^{+} S$-wave amplitude. In Fig. 4.3b we show, in the same mass range, the $K^{-} K^{+} P$-wave amplitude parametrized by the $\phi(1020)$ meson.

\subsection{Maximum likelihood fit to data}

To fit the Dalitz plot, we try several models incorporating various combinations of intermediate states. In each fit, we include the $K^{*}(892)^{+}$and measure the complex amplitude coefficients of other states relative to it. As a check on the quality of each fit, we compare the number of events observed in bins in the Dalitz plot with the number predicted by the fit. We compute residuals and statistical uncertainties to form a $\chi^{2}$, and take $\chi^{2} / \nu$ (where $\nu$ is the number of bins less the number of variable parameters) as a figure of merit. We also compare the distributions of angular moments (described later) predicted by the fit and actually observed in the data. 

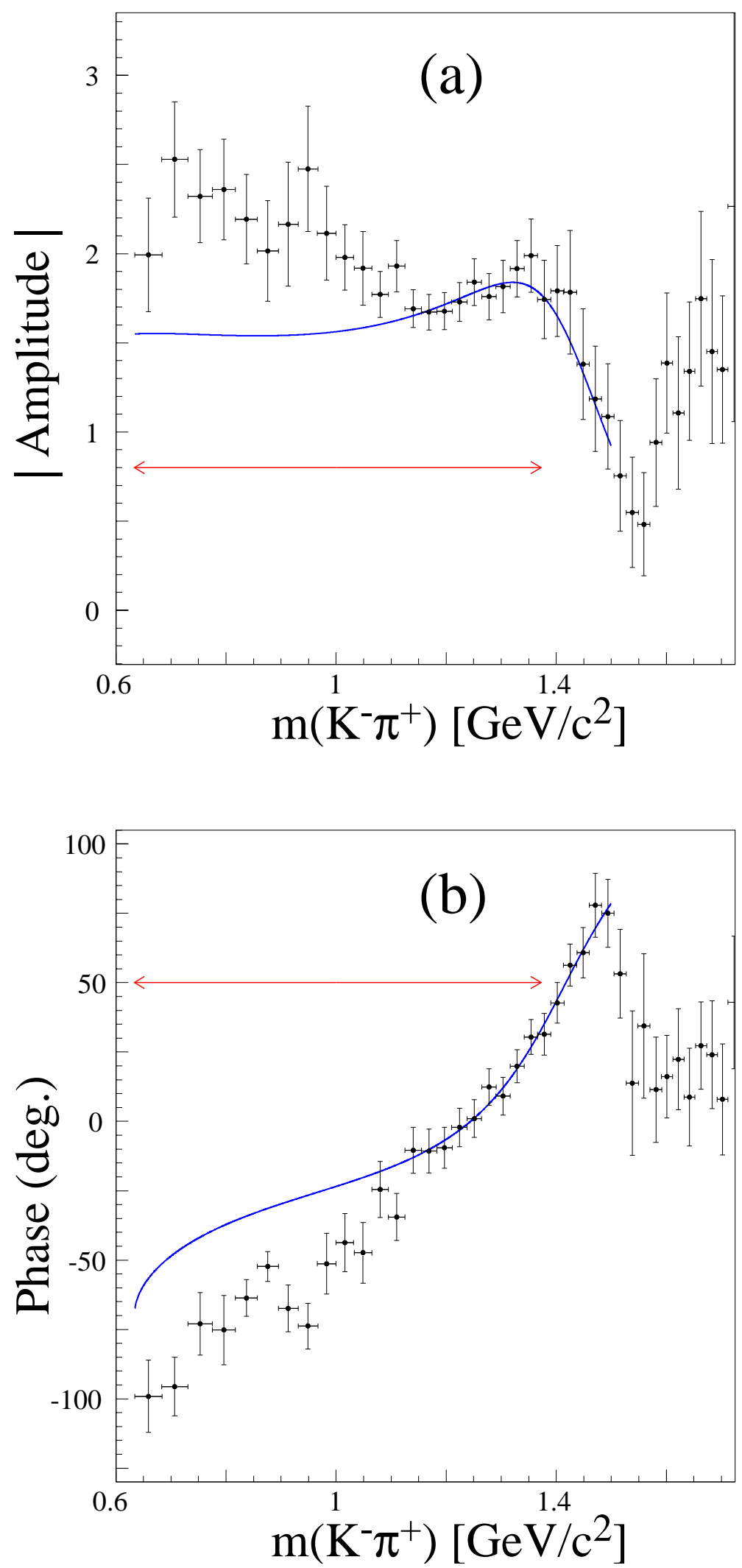

Figure 4.2: LASS (solid line, blue) and5\$-791 (dots with error bars) $K \pi S$-wave amplitudes (a), in arbitrary units, and phase (b). The double headed arrow (red) indicates the mass range available in the decay $D^{0} \rightarrow K^{-} K^{+} \pi^{0}$. 
Table 4.1: The results obtained from the $D^{0} \rightarrow K^{-} K^{+} \pi^{0}$ Dalitz plot fit. We define amplitude coefficients $a_{r}$ and $\phi_{r}$ relative to those of the $K^{*}(892)^{+}$. The errors are statistical and systematic, respectively. We show the $a_{0}(980)$ contribution, when it is included in place of the $f_{0}(980)$, in square brackets. We denote the $K \pi S$-wave states here by $K^{ \pm} \pi^{0}(S)$. We use LASS amplitude to describe the $K \pi S$-wave states in both the isobar models (I and II).

\begin{tabular}{|l|rrr|}
\hline & Model I & & \\
\hline State & Amplitude, $a_{r}$ & Phase, $\phi_{r}\left(^{\circ}\right)$ & Fraction, $f_{r}(\%)$ \\
\hline \hline$K^{*}(892)^{+}$ & 1.0 (fixed) & 0.0 (fixed) & $45.2 \pm 0.8 \pm 0.6$ \\
$K^{*}(1410)^{+}$ & $2.29 \pm 0.37 \pm 0.20$ & $86.7 \pm 12.0 \pm 9.6$ & $3.7 \pm 1.1 \pm 1.1$ \\
$K^{+} \pi^{0}(S)$ & $1.76 \pm 0.36 \pm 0.18$ & $-179.8 \pm 21.3 \pm 12.3$ & $16.3 \pm 3.4 \pm 2.1$ \\
$\phi(1020)$ & $0.69 \pm 0.01 \pm 0.02$ & $-20.7 \pm 13.6 \pm 9.3$ & $19.3 \pm 0.6 \pm 0.4$ \\
$f_{0}(980)$ & $0.51 \pm 0.07 \pm 0.04$ & $-177.5 \pm 13.7 \pm 8.6$ & $6.7 \pm 1.4 \pm 1.2$ \\
{$\left[a_{0}(980)^{0}\right]$} & {$[0.48 \pm 0.08 \pm 0.04]$} & {$[-154.0 \pm 14.1 \pm 8.6]$} & {$[6.0 \pm 1.8 \pm 1.2]$} \\
$f_{2}^{\prime}(1525)$ & $1.11 \pm 0.38 \pm 0.28$ & $-18.7 \pm 19.3 \pm 13.6$ & $0.08 \pm 0.04 \pm 0.05$ \\
$K^{*}(892)^{-}$ & $0.601 \pm 0.011 \pm 0.011$ & $-37.0 \pm 1.9 \pm 2.2$ & $16.0 \pm 0.8 \pm 0.6$ \\
$K^{*}(1410)^{-}$ & $2.63 \pm 0.51 \pm 0.47$ & $-172.0 \pm 6.6 \pm 6.2$ & $4.8 \pm 1.8 \pm 1.2$ \\
$K^{-} \pi^{0}(S)^{-}$ & $0.70 \pm 0.27 \pm 0.24$ & $133.2 \pm 22.5 \pm 25.2$ & $2.7 \pm 1.4 \pm 0.8$ \\
\hline & Model II & & \\
\hline$K^{*}(892)^{+}$ & 1.0 (fixed) & $0.0($ fixed) & $44.4 \pm 0.8 \pm 0.6$ \\
$K^{+} \pi^{0}(S)$ & $3.66 \pm 0.11 \pm 0.09$ & $-148.0 \pm 2.0 \pm 2.8$ & $71.1 \pm 3.7 \pm 1.9$ \\
$\phi(1020)$ & $0.70 \pm 0.01 \pm 0.02$ & $18.0 \pm 3.7 \pm 3.6$ & $19.4 \pm 0.6 \pm 0.5$ \\
$f_{0}(980)$ & $0.64 \pm 0.04 \pm 0.03$ & $-60.8 \pm 2.5 \pm 3.0$ & $10.5 \pm 1.1 \pm 1.2$ \\
{$\left[a_{0}(980)^{0}\right]$} & {$[0.68 \pm 0.06 \pm 0.03]$} & {$[-38.5 \pm 4.3 \pm 3.0]$} & {$[11.0 \pm 1.5 \pm 1.2]$} \\
$K^{*}(892)^{-}$ & $0.597 \pm 0.013 \pm 0.009$ & $-34.1 \pm 1.9 \pm 2.2$ & $15.9 \pm 0.7 \pm 0.6$ \\
$K^{-} \pi^{0}(S)$ & $0.85 \pm 0.09 \pm 0.11$ & $108.4 \pm 7.8 \pm 8.9$ & $3.9 \pm 0.9 \pm 1.0$ \\
\hline
\end{tabular}

Table 4.2: Parameter index for the correlation matrix.

\begin{tabular}{lr|lr|lr|lr}
\hline Index & Parameter & Index & Parameter & Index & Parameter & Index & Parameter \\
\hline 1 & $a_{f_{0}(980)}$ & 2 & $\phi_{f_{0}(980)}$ & 3 & $a_{f_{2}^{\prime}(1525)}$ & 4 & $\phi_{f_{2}^{\prime}(1525)}$ \\
5 & $a_{K^{*}(892)^{-}}$ & 6 & $\phi_{K^{*}(892)^{-}}$ & 7 & $a_{K^{*}(1410)^{+}}$ & 8 & $\phi_{K^{*}(1410)^{+}}$ \\
9 & $a_{K^{*}(1410)^{-}}$ & 10 & $\phi_{K^{*}(1410)^{-}}$ & 11 & $a_{K^{+} \pi^{0}(S)}$ & 12 & $\phi_{K^{+} \pi^{0}(S)}$ \\
13 & $a_{K^{-} \pi^{0}(S)}$ & 14 & $\phi_{K^{-} \pi^{0}(S)}$ & 15 & $a_{\phi(1020)}$ & 16 & $\phi_{\phi(1020)}$ \\
\hline
\end{tabular}


Table 4.3: Parameter correlation matrix for fit Model-I. The parameter indices are explained in Table 4.2 .

\begin{tabular}{c|cccccccc}
\hline No. & 1 & 2 & 3 & 4 & 5 & 6 & 7 & 8 \\
\hline 1 & 1.00 & -0.43 & -0.09 & 0.33 & 0.18 & -0.23 & 0.51 & -0.71 \\
2 & -0.43 & 1.00 & 0.04 & 0.03 & -0.23 & 0.55 & 0.18 & 0.58 \\
3 & -0.09 & 0.04 & 1.00 & -0.02 & -0.01 & 0.00 & -0.09 & 0.10 \\
4 & 0.33 & 0.03 & -0.02 & 1.00 & 0.12 & 0.07 & 0.34 & -0.27 \\
5 & 0.18 & -0.23 & -0.01 & 0.12 & 1.00 & -0.13 & 0.02 & -0.12 \\
6 & -0.23 & 0.55 & 0.00 & 0.07 & -0.13 & 1.00 & 0.16 & 0.47 \\
7 & 0.51 & 0.18 & -0.09 & 0.34 & 0.02 & 0.16 & 1.00 & -0.21 \\
8 & -0.71 & 0.58 & 0.10 & -0.27 & -0.12 & 0.47 & -0.21 & 1.00 \\
9 & 0.42 & -0.35 & 0.05 & 0.19 & 0.18 & -0.42 & 0.14 & -0.44 \\
10 & 0.55 & -0.09 & -0.12 & 0.23 & 0.33 & 0.06 & 0.26 & -0.29 \\
11 & -0.14 & 0.46 & -0.00 & 0.02 & -0.15 & 0.36 & 0.22 & 0.24 \\
12 & -0.43 & 0.50 & -0.02 & -0.05 & 0.01 & 0.58 & -0.00 & 0.56 \\
13 & -0.48 & -0.28 & 0.06 & -0.35 & 0.02 & -0.29 & -0.63 & 0.17 \\
14 & 0.57 & -0.13 & -0.08 & 0.30 & 0.14 & 0.08 & 0.39 & -0.32 \\
15 & -0.12 & -0.02 & 0.00 & -0.01 & 0.08 & -0.02 & -0.05 & 0.03 \\
16 & -0.33 & 0.59 & 0.06 & 0.11 & -0.17 & 0.33 & 0.05 & 0.34 \\
\hline No. & 9 & 10 & 11 & 12 & 13 & 14 & 15 & 16 \\
\hline 1 & 0.42 & 0.55 & -0.14 & -0.43 & -0.47 & 0.57 & -0.12 & -0.33 \\
2 & -0.35 & -0.09 & 0.46 & 0.50 & -0.28 & -0.13 & -0.02 & 0.59 \\
3 & 0.05 & -0.12 & -0.00 & -0.02 & 0.06 & -0.08 & 0.00 & 0.06 \\
4 & 0.19 & 0.23 & 0.02 & -0.05 & -0.35 & 0.30 & -0.01 & 0.11 \\
5 & 0.18 & 0.33 & -0.15 & 0.01 & 0.02 & 0.14 & 0.08 & -0.17 \\
6 & -0.42 & 0.06 & 0.36 & 0.58 & -0.29 & 0.08 & -0.02 & 0.33 \\
7 & 0.14 & 0.26 & 0.22 & -0.00 & -0.63 & 0.39 & -0.05 & 0.05 \\
8 & -0.44 & -0.29 & 0.24 & 0.56 & 0.17 & -0.32 & 0.03 & 0.34 \\
9 & 1.00 & 0.18 & -0.38 & -0.36 & -0.06 & 0.25 & 0.16 & 0.10 \\
10 & 0.18 & 1.00 & -0.06 & -0.07 & -0.33 & 0.41 & -0.04 & -0.03 \\
11 & -0.38 & -0.06 & 1.00 & 0.28 & -0.33 & 0.21 & -0.03 & 0.20 \\
12 & -0.36 & -0.07 & 0.28 & 1.00 & -0.22 & -0.11 & 0.07 & 0.41 \\
13 & -0.06 & -0.33 & -0.33 & -0.22 & 1.00 & -0.48 & 0.19 & -0.20 \\
14 & 0.25 & 0.41 & 0.21 & -0.11 & -0.48 & 1.00 & -0.16 & -0.08 \\
15 & 0.16 & -0.04 & -0.03 & 0.07 & 0.19 & -0.16 & 1.00 & 0.24 \\
16 & 0.10 & -0.03 & 0.20 & 0.41 & -0.20 & -0.08 & 0.24 & 1.00 \\
\hline & & & & & & & & \\
& & & & & \\
1 &
\end{tabular}



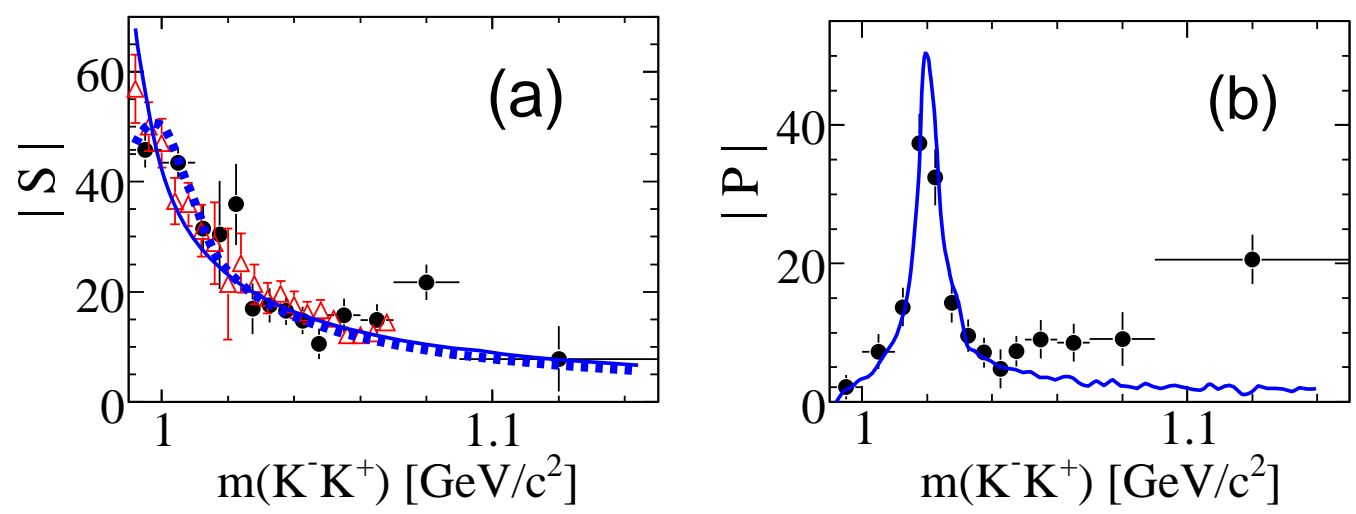

Figure 4.3: The phase-space-corrected $K^{-} K^{+} S$ - and $P$-wave amplitudes, $|S|$ and $|P|$ respectively, in arbitrary units, as functions of the invariant mass. (a) Lineshapes for (solid line, blue) $f_{0}(980)$, and (broken line, blue) $a_{0}(980)$, derived from Eq. 4.8. (b) Lineshape for $\phi(1020)$ (solid line, blue). In each plot, solid circles with error bars correspond to values obtained from the model-independent analysis for $|S|$ and $|P|$ using Eq. 4.9. In (a), the open triangles (red) correspond to values obtained from the decay $D^{0} \rightarrow K^{-} K^{+} \bar{K}^{0}$ (see text).

Table 4.4: Parameter correlation matrix for fit Model-II. Parameters with indices 1-4 of Table 4.2 are not included in this fit model.

\begin{tabular}{c|cccccccccccc}
\hline NO. & 5 & 6 & 7 & 8 & 9 & 10 & 11 & 12 & 13 & 14 & 15 & 16 \\
\hline 5 & 1.00 & -0.42 & 0.42 & 0.19 & -0.07 & 0.38 & 0.48 & 0.01 & 0.07 & 0.34 & 0.31 & 0.27 \\
6 & -0.42 & 1.00 & -0.46 & -0.11 & 0.10 & -0.21 & -0.40 & 0.08 & -0.03 & -0.22 & -0.13 & -0.20 \\
7 & 0.42 & -0.46 & 1.00 & 0.32 & -0.38 & 0.62 & 0.83 & 0.12 & 0.04 & 0.75 & 0.06 & 0.69 \\
8 & 0.19 & -0.11 & 0.32 & 1.00 & -0.62 & -0.24 & 0.44 & 0.78 & -0.72 & 0.67 & -0.12 & 0.59 \\
9 & -0.07 & 0.10 & -0.38 & -0.62 & 1.00 & 0.12 & -0.54 & -0.70 & 0.56 & -0.71 & 0.23 & -0.46 \\
10 & 0.38 & -0.21 & 0.62 & -0.24 & 0.12 & 1.00 & 0.49 & -0.45 & 0.54 & 0.25 & 0.26 & 0.36 \\
11 & 0.48 & -0.40 & 0.83 & 0.44 & -0.54 & 0.49 & 1.00 & 0.21 & 0.02 & 0.87 & 0.17 & 0.62 \\
12 & 0.01 & 0.08 & 0.12 & 0.78 & -0.70 & -0.45 & 0.21 & 1.00 & -0.90 & 0.57 & -0.29 & 0.49 \\
13 & 0.07 & -0.03 & 0.04 & -0.72 & 0.56 & 0.54 & 0.02 & -0.90 & 1.00 & -0.33 & 0.32 & -0.38 \\
14 & 0.34 & -0.22 & 0.75 & 0.67 & -0.71 & 0.25 & 0.87 & 0.57 & -0.33 & 1.00 & -0.08 & 0.74 \\
15 & 0.31 & -0.13 & 0.06 & -0.12 & 0.23 & 0.26 & 0.17 & -0.29 & 0.32 & -0.08 & 1.00 & 0.00 \\
16 & 0.27 & -0.20 & 0.69 & 0.59 & -0.46 & 0.36 & 0.62 & 0.49 & -0.38 & 0.74 & 0.00 & 1.00 \\
\hline
\end{tabular}




\subsection{Result on $K \pi S$-wave}

The LASS $K \pi S$-wave amplitude gives the best agreement with data and we use it in our nominal fits (see next paragraph). The $K \pi S$-wave modeled by the combination of $\kappa(800)$ (with parameters taken from Ref. [23]), a nonresonant term and $K_{0}^{*}(1430)$ has a smaller fit probability $\left(\chi^{2}\right.$ probability $\left.<5 \%\right)$. The best fit with this model $\left(\chi^{2}\right.$ probability $13 \%)$ yields a charged $\kappa$ of mass $(870 \pm 30) \mathrm{MeV} / c^{2}$, and width $(150 \pm$ 20) $\mathrm{MeV} / c^{2}$, significantly different from those reported in Ref. [23] for the neutral state. This does not support the hypothesis that production of a charged, scalar $\kappa$ is being observed. The E-791 amplitude [30] describes the data well, except near threshold ( $\chi^{2}$ probability $23 \%$ ). Though our data favor the LASS parametrization for $\sqrt{s}<1.15 \mathrm{GeV} / c^{2}$, the insensitivity of the fit to small variations in amplitude at these masses does not allow an independent $S$-wave measurement with the present data sample. Therefore, we use the E-791 amplitude to estimate systematic uncertainty in our results.

\subsection{Results of the Dalitz plot fit}

We find that two different isobar models describe the data well. Both yield almost identical behavior in invariant mass (Fig. 4.1b-4.1d) and angular distribution (Fig. 4.4). We use LASS amplitude to describe the $K \pi S$-wave amplitudes in both the isobar models (I and II). We summarize the results of the best fits (Model I: $\chi^{2} / \nu=702.08 / 714$, probability $61.9 \%$; Model II: $\chi^{2} / \nu=718.89 / 717$, probability $47.3 \%)$ in Table 4.1. We also list the fit fraction for each resonant process $r$, defined as $f_{r} \equiv \int\left|a_{r} A_{r}\right|^{2} d \tau / \int\left|f_{D^{0}}\right|^{2} d \tau$, where $d \tau=d m_{K^{-} \pi^{0}}^{2} d m_{K^{+} \pi^{0}}^{2}$, in Table 4.1. Due to interference among the contributing amplitudes, the $f_{r}$ do not sum to one in general. We find that the $K \pi S$-wave is not in phase with the $P$-wave at threshold as it was in the LASS scattering data. For Model I (II), the $S$-wave phase relative to the $K^{*}(892)^{+}$is $\sim 180^{\circ}\left(150^{\circ}\right)$ for the positive charge and $135^{\circ}\left(110^{\circ}\right)$ for the negative charge. We give the correlation matrix for the parametrization coefficients of the $D^{0} \rightarrow K^{-} K^{+} \pi^{0}$ Dalitz plot fit in Tables 4.2-4.4.

\subsection{Are there additional states ?}

We have also considered the possible contributions from other resonant states such as: $K_{2}^{*}(1430), f_{2}(1270), f_{0}(1370)$, and $f_{0}(1510)$. We find that none of them is needed to describe the Dalitz plot, they all provide small contributions and lead to smaller $\chi^{2}$ probabilities.

\subsection{Description of angular moments}

Angular distributions provide a more detailed information on specific features of the amplitudes used in the description of the Dalitz plot. We define the helicity angle $\theta_{H}$ for the decay $D^{0} \rightarrow(r \rightarrow A B) C$ as the angle between the momentum of $A$ in the $A B$ rest frame and the momentum of $A B$ in the $D^{0}$ rest frame. The 

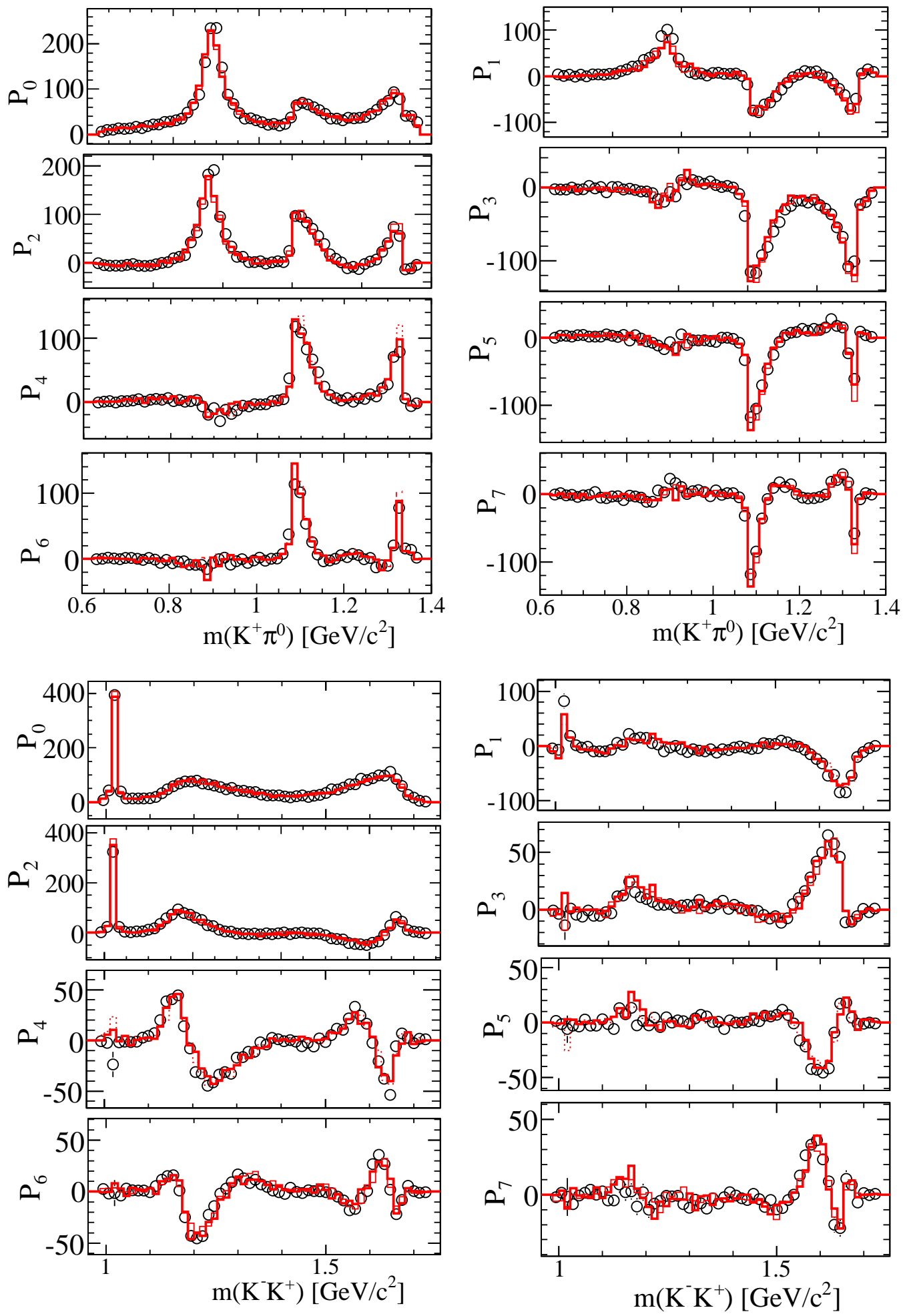

Figure 4.4: The mass dependence of the spherical harmonic moments of $\cos \theta_{H}$ after efficiency corrections and background subtraction: $K^{+} \pi^{0}$ (top) and $K^{-} K^{+}$ (bottom). The circles with error bars are data points and the curves (red) are derived from the fit functions (see text). For the sake of visibility, we do not show error bars on the curves. 
moments of $\cos \theta_{H}$, defined as the efficiency-corrected and background-subtracted invariant mass distributions of events weighted by spherical harmonic functions,

$Y_{l}^{0}\left(\theta_{H}\right)=\sqrt{\frac{2 l+1}{4 \pi}} P_{l}\left(\cos \theta_{H}\right)$, where the $P_{l}$ are Legendre polynomials of order $l$, are shown in Fig. 4.4 for the $K^{+} \pi^{0}$ and $K^{-} K^{+}$channels, for $l=0-7$. The $K^{-} \pi^{0}$ moments are similar to those for $K^{+} \pi^{0}$.

\subsection{Model-independent partial wave analysis}

The mass dependent $K^{-} K^{+} S$ - and $P$-wave complex amplitudes can also be obtained directly from our data in a model-independent way in a limited mass range around $1 \mathrm{GeV} / c^{2}$. In a region of the Dalitz plot where $S$ - and $P$-waves in a single channel dominate, their amplitudes are given by the following Legendre polynomial moments,

$$
\begin{aligned}
& P_{0}=\frac{|S|^{2}+|P|^{2}}{\sqrt{2}}, \\
& P_{1}=\sqrt{2}|S||P| \cos \theta_{S P}, \\
& P_{2}=\sqrt{\frac{2}{5}}|P|^{2},
\end{aligned}
$$

using $\int_{-1}^{1} P_{l} P_{m} d\left(\cos \theta_{H}\right)=\delta_{l m}$. Here $|S|$ and $|P|$ are, respectively, the magnitudes of the $S$ - and $P$-wave amplitudes, and $\theta_{S P}=\theta_{S}-\theta_{P}$ is the relative phase between them. We use these relations to evaluate $|S|$ and $|P|$, shown in Fig. 4.3, for the $K^{-} K^{+}$channel in the mass range $m_{K^{-} K^{+}}<1.15 \mathrm{GeV} / c^{2}$. The measured values of $|S|$ agree well with those obtained in the analysis of the decay $D^{0} \rightarrow K^{-} K^{+} \bar{K}^{0}$ [34]. They also agree well with either the $f_{0}(980)$ or the $a_{0}(980)$ lineshape. The measured values of $|P|$ are consistent with a Breit-Wigner lineshape for $\phi(1020)$. Results for $\cos \theta_{S P}$ and $\theta_{S P}$ are shown in Figs. 4.5a-4.5b. A twofold ambiguity in the sign of $\theta_{S P}$ exists, as shown in Fig. 4.5b. It is, however, straightforward to choose the physical solution. In this region, the $\phi(1020)$ meson $(P$-wave) has a very rapidly rising phase, while we expect the $S$-wave phase to be relatively slowly varying. Thus, the upper solution, in which $\theta_{S}-\theta_{P}$ is rapidly falling, is the physical solution. We take the Breit-Wigner phase of $\phi(1020)$, shown in Fig. 4.5c, to be a good model for $\theta_{P}$ and obtain $\theta_{S}$, as plotted in Fig. 4.5d. These results show little variation in $S$-wave phase up to about $1.02-1.03 \mathrm{GeV} / c^{2}$, then a rapid rise above that. Also, in Fig. 4.3b, we observe that $|P|$ follows the $\phi(1020)$ curve well up to about the same mass, with a significant deviation above that. The behavior observed matches well to that obtained from the isobar model I or II. No distinction between them appears possible from this analysis. The partial-wave analysis described above is valid, in the absence of higher spin states, only if no interference occurs from the crossing $K \pi$ channels. The behavior observed in both $S$ - and $P$-waves above $\sim 1.03 \mathrm{GeV} / c^{2}$ can, therefore, be attributed to high mass tails of the $K^{*}(892)$ and low mass tails of possible higher $K^{*}$ resonances. 

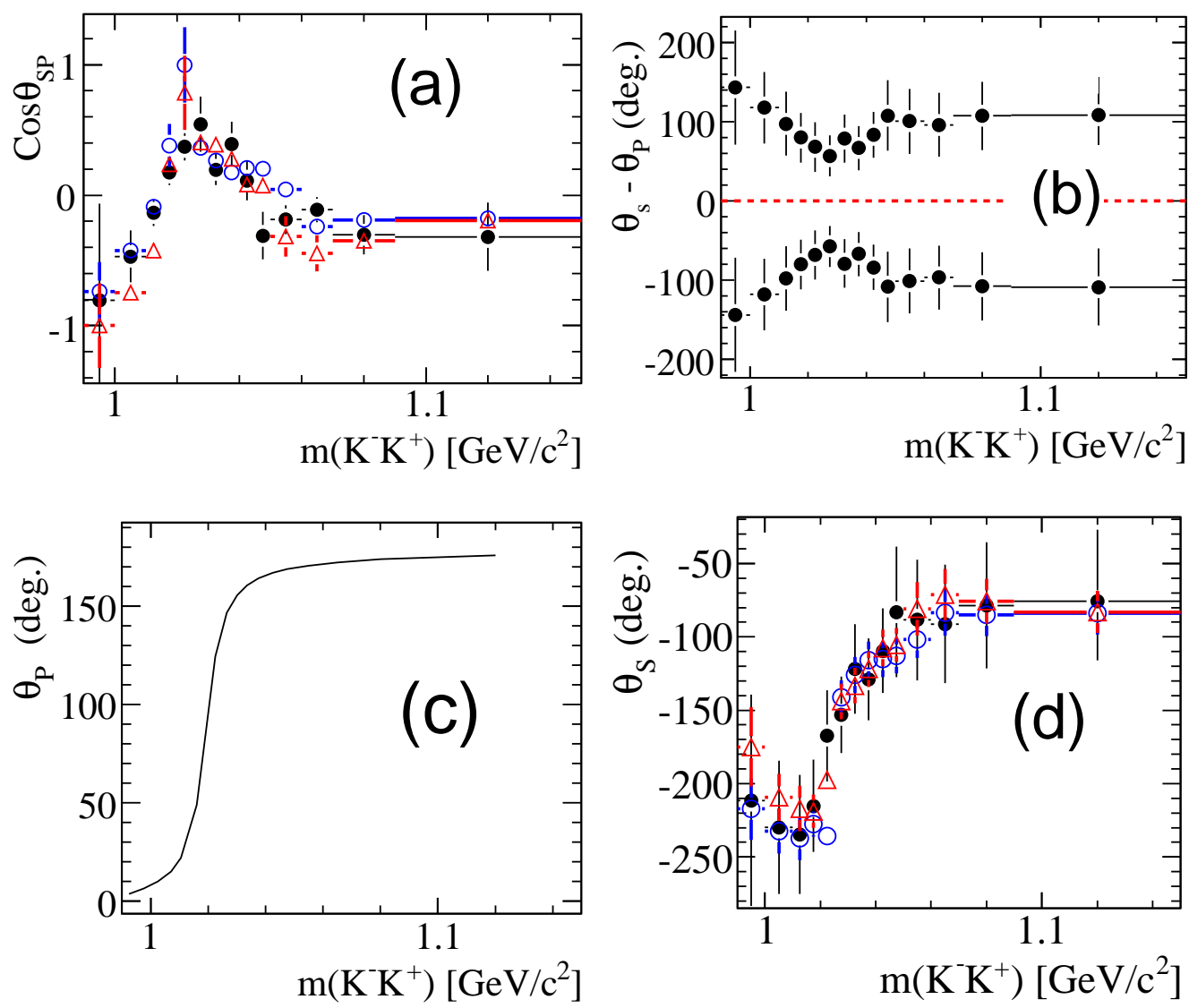

Figure 4.5: Results of the partial-wave analysis of the $K^{-} K^{+}$system using Eq. 4.9 described in the text. (a) Cosine of relative phase $\theta_{S P}=\theta_{S}-\theta_{P}$, (b) two solutions for $\theta_{S P}$, (c) $P$-wave phase taken from Eqs. 4.4-4.5 for the $\phi(1020)$ meson, and (d) $S$-wave phase derived from the upper solution in (b). Solid bullets are data points, and open circles (blue) and open triangles (red) correspond, respectively, to isobar models I and II. The number of simulated events used for the two models is 10 times larger than data. Errors for quantities from the isobar models arise from Monte Carlo statistical limitations, and differ from errors derived from Eq. 4.9. 


\subsection{Systematic uncertainties}

Systematic uncertainties in quantities in Table 4.1 arise from experimental effects, and also from uncertainties in the nature of the models used to describe the data. We determine these separately and add them in quadrature. In both cases, we assign the maximum deviation in the observed quantities (i.e., $a_{r}, \phi_{r}$, and $f_{r}$ ) from the central value as a systematic uncertainty, taking correlations among fit parameters into account. We characterize the uncertainties due to $K \pi S$-wave amplitudes and resonance mass-width values as model dependent. We estimate them conservatively taking symmetric errors from the spread in results when either the LASS amplitude is replaced by the E-791 amplitude, or the resonance parameters are changed by one standard deviation $(\sigma)$. Similarly, we estimate the experimental uncertainty from the variation in results when either the signal efficiency parameters are varied by $1 \sigma$, or the background shape is taken from simulation instead of the data sideband, or the ratio of particle-identification rates in data and simulation is varied by $1 \sigma$. Model and experimental systematics contribute almost equally to the total uncertainty. As a consistency check, we analyze disjoint data samples, in bins of reconstructed $D^{0}$ mass and laboratory momentum, and find consistent results.

\subsection{Strong-phase difference and suppression factor}

Neglecting $C P$ violation, the strong phase difference, $\delta_{D}$, between the $\bar{D}^{0}$ and $D^{0}$ decays to $K^{*}(892)^{+} K^{-}$state and their amplitude ratio, $r_{D}$, are given by

$$
r_{D} e^{i \delta_{D}}=\frac{a_{D^{0} \rightarrow K^{*-} K^{+}}}{a_{D^{0} \rightarrow K^{*+} K^{-}}} e^{i\left(\delta_{K^{*-} K^{+}}-\delta_{K^{*+} K^{-}}\right)} .
$$

Combining the results of models I and II, we find $\delta_{D}=-35.5^{\circ} \pm 1.9^{\circ}$ (stat) $\pm 2.2^{\circ}$ (syst) and $r_{D}=0.599 \pm 0.013$ (stat) \pm 0.011 (syst). These results are consistent with the previous measurements [35], $\delta_{D}=-28^{\circ} \pm 8^{\circ}$ (stat) $\pm 11^{\circ}$ (syst) and $r_{D}=$ $0.52 \pm 0.05$ (stat) \pm 0.04 (syst).

\subsection{Summary}

In conclusion, we have studied the amplitude structure of the decay $D^{0} \rightarrow K^{-} K^{+} \pi^{0}$, and measured $\delta_{D}$ and $r_{D}$. We find that two isobar models give excellent descriptions of the data. Both models include significant contributions from $K^{*}(892)$, and each indicates that $D^{0} \rightarrow K^{*+} K^{-}$dominates over $D^{0} \rightarrow K^{*-} K^{+}$. This suggests that, in tree-level diagrams, the form factor for $D^{0}$ coupling to $K^{*-}$ is suppressed compared to the corresponding $K^{-}$coupling. While the measured fit fraction for $D^{0} \rightarrow K^{*+} K^{-}$agrees well with a phenomenological prediction [36] based on a large $\mathrm{SU}(3)$ symmetry breaking, the corresponding results for $D^{0} \rightarrow K^{*-} K^{+}$and the color-suppressed $D^{0} \rightarrow \phi \pi^{0}$ decays differ significantly from the predicted values. It appears from Table 4.1 that the $K^{+} \pi^{0} S$-wave amplitude can absorb any $K^{*}(1410)$ and $f_{2}^{\prime}(1525)$ if those are not in the model. The other components are quite well established, independent of the model. The $K \pi S$-wave amplitude is consistent with 
that from the LASS analysis, throughout the available mass range. We cannot, however, completely exclude the behavior at masses below $\sim 1.15 \mathrm{GeV} / c^{2}$ observed in the decay $D^{+} \rightarrow K^{-} \pi^{+} \pi^{+}[23,30]$. The $K^{-} K^{+} S$-wave amplitude, parametrized as either $f_{0}(980)$ or $a_{0}(980)^{0}$, is required in both isobar models. No higher mass $f_{0}$ states are found to contribute significantly. In a limited mass range, from threshold up to $1.02 \mathrm{GeV} / c^{2}$, we measure this amplitude using a model-independent partial-wave analysis. Agreement with similar measurements from $D^{0} \rightarrow K^{-} K^{+} \bar{K}^{0}$ decay [34], and with the isobar models considered here, is excellent. 


\section{Chapter 5}

\section{Amplitude Analysis of the Decay $D^{0} \rightarrow \pi^{-} \pi^{+} \pi^{0}$}

\subsection{Data and simulation samples}

Using $232 \mathrm{fb}^{-1}$ of $e^{+} e^{-}$collision data at the center-of-mass energies of 10.58 and $10.54 \mathrm{GeV}$ with the BABAR detector [12] at the PEP-II storage rings, we study the amplitudes of the singly Cabibbo-suppressed decay $D^{0} \rightarrow \pi^{-} \pi^{+} \pi^{0}$ [25]. We measure the strong phase difference between the $\bar{D}^{0}$ and $D^{0}$ decays to $\rho(770)^{+} \pi^{-}$to be $-2.0^{\circ} \pm 0.6^{\circ}$ (stat) $\pm 0.6^{\circ}$ (syst), and their amplitude ratio to be $0.714 \pm 0.008$ (stat) \pm 0.003 (syst). We observe contributions from the $\pi \pi$ scalar and vector amplitudes, and analyze their angular moments.

For selection optimizations, we use the generic simulation samples of $e^{+} e^{-} \rightarrow$ $b \bar{b}\left(2045 \mathrm{fb}^{-1}\right), c \bar{c}\left(327.5 \mathrm{fb}^{-1}\right)$ and also $u \bar{u}, d \bar{d}, s \bar{s}\left(322.5 \mathrm{fb}^{-1}\right)$. In addition, $D^{0} \rightarrow$ $\pi^{-} \pi^{+} \pi^{0}$ signal events (4.7 million) generated uniformly in phase space from $c \bar{c}$ events are also used to study detector acceptance and relative signal efficiency.

\subsection{Event selection}

We distinguish $D^{0}$ from $\bar{D}^{0}$ by reconstructing the decays $D^{*+} \rightarrow D^{0} \pi^{+}$and $D^{*-} \rightarrow$ $\bar{D}^{0} \pi^{-}$. The event-selection criteria are the same as those used in our measurement of the branching ratio of the decay $D^{0} \rightarrow \pi^{-} \pi^{+} \pi^{0}$ [18] described in Chapter 3 .. In particular, we require that the $\mathrm{CM}$ momentum of $D^{0}$ candidate be greater than $2.77 \mathrm{GeV} / c$, and that $\left|m_{D^{*+}}-m_{D^{0}}-145.4\right|<0.6 \mathrm{MeV} / c^{2}$, where $m$ refers to a reconstructed invariant mass. We exclude the decay mode $D \rightarrow K_{S}^{0} \pi^{0}$, which is a Cabibbo-favored $C P$ eigenstate not related to the present study, by rejecting candidates with $489<M\left(\pi^{+} \pi^{-}\right)<508 \mathrm{MeV} / c^{2}$ or for which the distance between the $\pi^{+} \pi^{-}$vertex and the $D^{*}$ candidate decay vertex is more than $1.5 \mathrm{~cm}$. To minimize uncertainty from background shape, we choose a sample of very high purity $(\sim$ $98.1 \%$ ) using $1848<m_{D}<1880 \mathrm{MeV} / c^{2}$, and find $N_{S}=44780 \pm 250$ signal and $N_{B}=830 \pm 70$ background events. We estimate the signal efficiency for each event as a function of its position in the Dalitz plot using simulated $D^{0} \rightarrow \pi^{-} \pi^{+} \pi^{0}$ events from $c \bar{c}$ decays, generated uniformly in the available phase space. To correct for 
differences in particle-identification rates in data and simulation, we determine the ratio of these for each track, and apply an event-by-event correction factor.

\subsection{Efficiency calculation}

To obtain the efficiency function, a simulation sample of about 4.6 million $D^{0} \rightarrow$ $\pi^{-} \pi^{+} \pi^{0}$ signal events uniformly populated in phase space was generated. A track parameter-dependent particle-identification correction is applied to this sample on an event-by-event basis, using the ratio of signal reconstruction efficiencies in data and simulation in bins of momentum and polar angle. For this purpose, the generated and reconstructed Dalitz plots are divided into $\mathrm{N} \times \mathrm{N}$ cells, $\mathrm{N}$ chosen in such a way to have at least 25 reconstructed events per cell on average. The efficiency map is then obtained by dividing the reconstructed Dalitz plot over the generated one. The efficiency is parametrized as a 3rd order polynomial in the two Dalitz plot variables

$$
\begin{aligned}
\epsilon\left(s_{+}, s_{-}\right)=1 & +s_{1}\left(s_{+}+s_{-}\right) \\
& +s_{2}\left(s_{+}^{2}+s_{-}^{2}\right)+s_{3}\left(s_{+}^{3}+s_{-}^{3}\right) \\
& +s_{4}\left(s_{-} s_{+}^{2}+s_{+} s_{-}^{2}\right)+s_{5}\left(s_{+} s_{-}\right) .
\end{aligned}
$$

In addition, $\epsilon\left(s_{+}, s_{-}\right) \equiv 0$ for all points outside the physical boundary of the Dalitz plot. The results of the fit are

$s_{1}=3.56 \pm 0.25$

$s_{2}=-1.18 \pm 0.20$

$s_{3}=0.08 \pm 0.05$

$s_{4}=0.38 \pm 0.13$

$s_{5}=-2.15 \pm 0.38$

\subsection{Background model for Dalitz plot}

The Dalitz plot PDF for the background events is obtained from the sideband $1.930<$ $M\left(\pi^{-} \pi^{+} \pi^{0}\right)<1.990 \mathrm{GeV} / c^{2}$. To justify this procedure, we did a feasibility study to find out whether the background events in the signal region and the sideband have consistent Dalitz plot distributions. We proceeded in two steps:

- First, we compared the Dalitz plot distributions for events in the (upper) sideband of $m_{D^{0}}$ in data and simulation. We find that there is good agreement between them.

- Next, we compared the Dalitz plot distributions of the background events in the signal region and in the sideband for generic $q \bar{q}$ simulation events. Again, the agreement is very good.

Thus, we have verified that there is a good agreement between Dalitz plot distributions of the background events in the signal region and in the sideband, and the background shape can be taken from the sideband. 


\subsection{Fitting simulated events}

We conducted simulation experiments using CLEO parameters [41] for $D^{0} \rightarrow \pi^{-} \pi^{+} \pi^{0}$ decay. The invariant mass projections for one experiment are shown in Fig. 5.1. The pull distributions for the fit parameters are also shown. Fig. 5.2 shows the Dalitz plot variable projections for an experiment that also contains background.
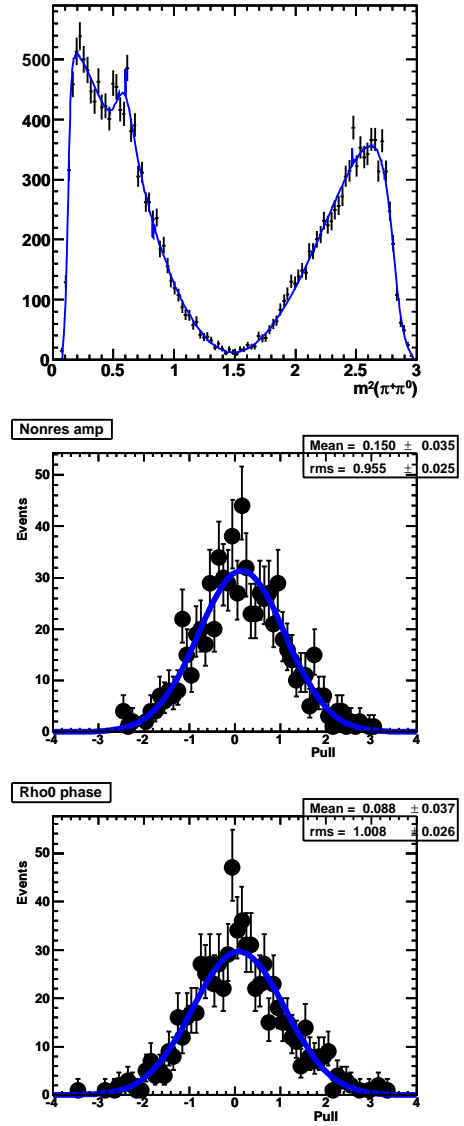
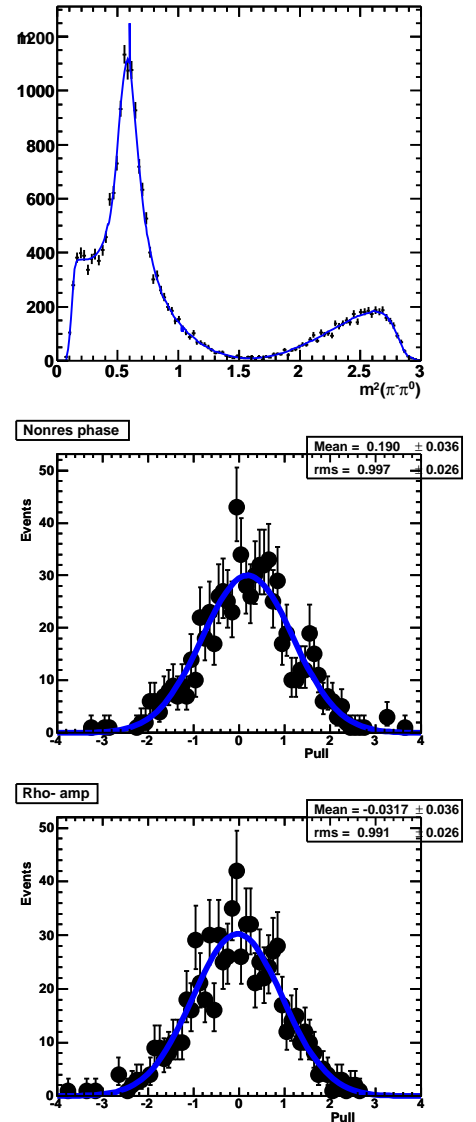
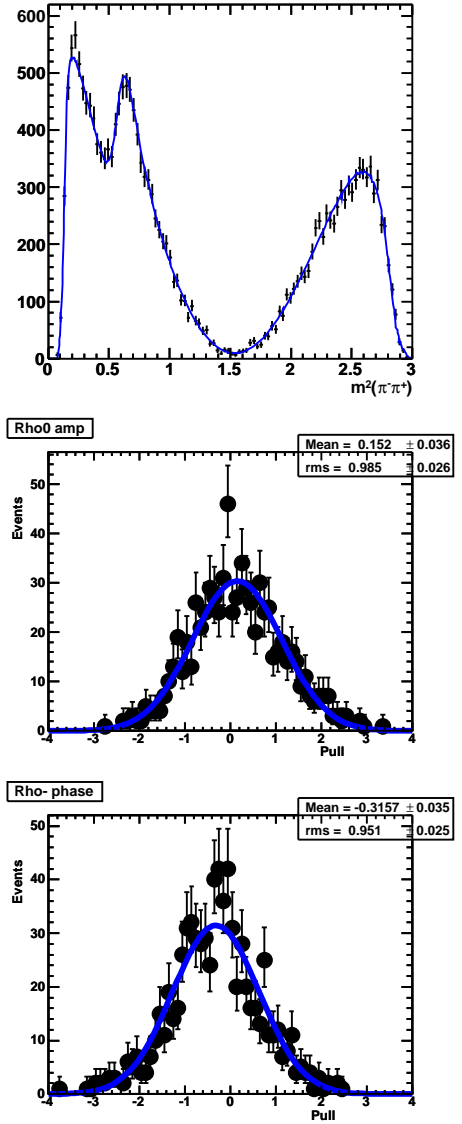

Figure 5.1: (Row 1) Invariant-mass projections for $\pi^{-} \pi^{+} \pi^{0}$ simulated events generated with CLEO parameters of Ref. [41] and fitted with a sum of three Breit-Wigners for $\rho(770)$ states. (Rows 2-3) Pull distributions for the fit parameters.

\subsection{Dalitz plot fit for data}

The results of the Dalitz plot fit to the data are shown in Table 5.1. The amplitude of the $\rho(770)^{+}$component is fixed to 1 and its phase is fixed to 0 (i.e., all amplitudes and phases are measured with reference to $\left.\rho(770)^{+}\right)$. In addition to the fit parameters, 

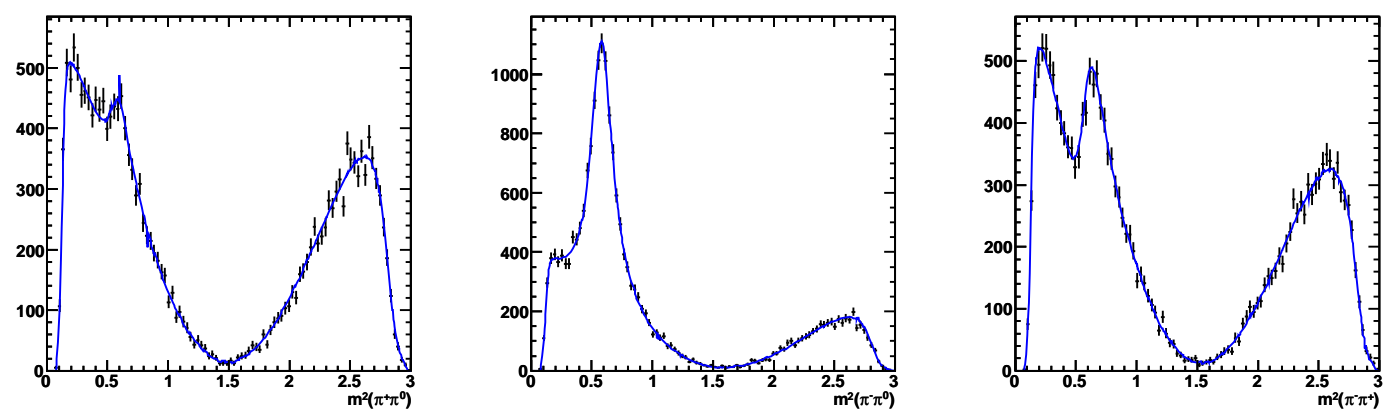

Figure 5.2: $\pi^{-} \pi^{+} \pi^{0}$ Dalitz plot for events generated with CLEO parameters and fitted with a sum of three Breit-Wigner amplitudes for the three $\rho(770)$ states. Background generated and fitted with a shape taken from Data sideband.

we also show the "fit fraction" for each PDF component $r$, defined as

$$
F_{r}=\frac{\int\left|a_{r} A_{r}\left(s_{+}, s_{-}\right) d s_{-} d s_{+}\right|^{2}}{\int\left|f_{D^{0}}\left(s_{+}, s_{-}\right)\right|^{2} d s_{-} d s_{+}} .
$$

Due to interference among the contributing states, the fit fractions do not sum to one in general. The efficiency-corrected Dalitz plot and invariant mass-squared projections are shown in Fig. 5.3.
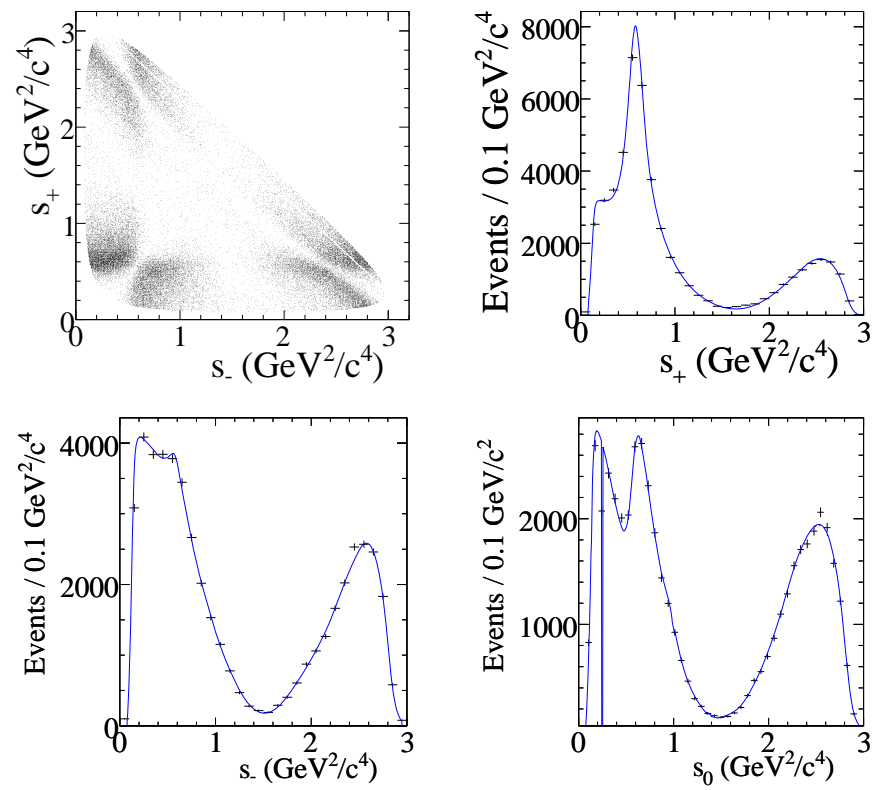

Figure 5.3: Efficiency-corrected Dalitz plot and invariant mass-squared projections for the $D^{0} / \bar{D}^{0} \rightarrow \pi^{-} \pi^{+} \pi^{0}$ decay excluding $D^{0} / \bar{D}^{0} \rightarrow K_{s}^{0} \pi^{0}$. 
Table 5.1: Result of the fit to the $D^{0} \rightarrow \pi^{-} \pi^{+} \pi^{0}$ Dalitz plot distribution. The amplitudes and phases are defined relative to those of the $\rho^{+}$. The fraction is $f_{r} \equiv \int\left|a_{r} A_{r}\right|^{2} d s_{-} d s_{+} / \int\left|f_{D^{0}}\right|^{2} d s_{-} d s_{+}$. The errors are statistical and systematic, respectively. We take the mass (width) of the $\sigma$ meson to be 400 (600) $\mathrm{MeV} / c^{2}$. The $\chi^{2} / \nu$ for the fit is 1.38 for $n u=565$.

\begin{tabular}{l|r|r|r}
\hline \hline State & Amplitude $a_{r}$ & Phase $\phi_{r}$ & Fraction $f_{r}(\%)$ \\
\hline$\rho^{+}(770)$ & 1 & 0 & $67.8 \pm 0.0 \pm 0.6$ \\
\hline$\rho^{0}(770)$ & $0.588 \pm 0.006 \pm 0.002$ & $16.2 \pm 0.6 \pm 0.4$ & $26.2 \pm 0.5 \pm 1.1$ \\
\hline$\rho^{-}(770)$ & $0.714 \pm 0.008 \pm 0.003$ & $-2.0 \pm 0.6 \pm 0.6$ & $34.6 \pm 0.8 \pm 0.3$ \\
\hline$\rho^{+}(1450)$ & $0.21 \pm 0.06 \pm 0.13$ & $-146 \pm 18 \pm 24$ & $0.11 \pm 0.07 \pm 0.12$ \\
\hline$\rho^{0}(1450)$ & $0.33 \pm 0.06 \pm 0.04$ & $10 \pm 8 \pm 13$ & $0.30 \pm 0.11 \pm 0.07$ \\
\hline$\rho^{-}(1450)$ & $0.82 \pm 0.05 \pm 0.04$ & $16 \pm 3 \pm 3$ & $1.79 \pm 0.22 \pm 0.12$ \\
\hline$\rho^{+}(1700)$ & $2.25 \pm 0.18 \pm 0.14$ & $-17 \pm 2 \pm 3$ & $4.1 \pm 0.7 \pm 0.7$ \\
\hline$\rho^{0}(1700)$ & $2.51 \pm 0.15 \pm 0.13$ & $-17 \pm 2 \pm 2$ & $5.0 \pm 0.6 \pm 1.0$ \\
\hline$\rho^{-}(1700)$ & $2.00 \pm 0.11 \pm 0.07$ & $-50 \pm 3 \pm 3$ & $3.2 \pm 0.4 \pm 0.6$ \\
\hline$f_{0}(980)$ & $0.052 \pm 0.004 \pm 0.006$ & $-59 \pm 5 \pm 4$ & $0.25 \pm 0.04 \pm 0.04$ \\
\hline$f_{0}(1370)$ & $0.22 \pm 0.03 \pm 0.03$ & $156 \pm 9 \pm 6$ & $0.37 \pm 0.11 \pm 0.09$ \\
\hline$f_{0}(1500)$ & $0.20 \pm 0.02 \pm 0.02$ & $12 \pm 9 \pm 4$ & $0.39 \pm 0.08 \pm 0.07$ \\
\hline$f_{0}(1710)$ & $0.39 \pm 0.05 \pm 0.06$ & $51 \pm 8 \pm 7$ & $0.31 \pm 0.07 \pm 0.08$ \\
\hline$f_{2}(1270)$ & $0.30 \pm 0.01 \pm 0.06$ & $-171 \pm 3 \pm 4$ & $1.32 \pm 0.08 \pm 0.10$ \\
\hline$\sigma$ & $0.24 \pm 0.02 \pm 0.04$ & $8 \pm 4 \pm 8$ & $0.82 \pm 0.10 \pm 0.10$ \\
\hline Nonres & $0.57 \pm 0.07 \pm 0.08$ & $-11 \pm 4 \pm 2$ & $0.84 \pm 0.21 \pm 0.12$ \\
\hline \hline
\end{tabular}

\subsection{Parameter-correlation matrix}

In this section we give the correlation matrix for the parametrization coefficients of the $D^{0} \rightarrow \pi^{-} \pi^{+} \pi^{0}$ Dalitz plot, the results for which were shown in the previous section. The matrix index is as follows:

Table 5.2: Parameter index for the correlation matrix.

\begin{tabular}{lr|lr|lr|lr}
\hline Index & Parameter & Index & Parameter & Index & Parameter & Index & Parameter \\
\hline 0 & $a_{f_{0}(1370)}$ & 1 & $\phi_{f_{0}(1370)}$ & 2 & $a_{f_{0}(1500)}$ & 3 & $\phi_{f_{0}(1500)}$ \\
4 & $a_{f_{0}(1710)}$ & 5 & $\phi_{f_{0}(1710)}$ & 6 & $a_{f_{0}(980)}$ & 7 & $\phi_{f_{0}(980)}$ \\
8 & $a_{f_{2}(1270)}$ & 9 & $\phi_{f_{2}(1270)}$ & 10 & $a_{\text {Nonres }}$ & 11 & $\phi_{\text {Nonres }}$ \\
12 & $a_{\rho(770)^{-}}$ & 13 & $\phi_{\rho(770)^{-}}$ & 14 & $a_{\rho(770)^{0}}$ & 15 & $\phi_{\rho(770)^{0}}$ \\
16 & $a_{\rho(1700)^{+}}$ & 17 & $\phi_{\rho(1700)^{+}}$ & 18 & $a_{\rho(1700)^{-}}$ & 19 & $\phi_{\rho(1700)^{-}}$ \\
20 & $a_{\rho(1700)^{0}}$ & 21 & $\phi_{\rho(1700)^{0}}$ & 22 & $a_{\rho(1450)^{+}}$ & 23 & $\phi_{\rho(1450)^{+}}$ \\
24 & $a_{\rho(1450)^{-}}$ & 25 & $\phi_{\rho(1450)^{-}}$ & 26 & $a_{\rho(1450)^{0}}$ & 27 & $\phi_{\rho(1450)^{0}}$ \\
28 & $a_{\sigma}$ & 29 & $\phi_{\sigma}$ & & & & \\
\hline
\end{tabular}


$30 \times 30$ matrix is as follows

\begin{tabular}{r|rrrrrr} 
& $\mid$ & 0 & 1 & 2 & 3 & 4 \\
-0 & $\mid$ & 1 & -0.2356 & 0.8357 & 0.1554 & 0.5407 \\
\hline 1 & -0.2356 & 1 & -0.5204 & 0.7713 & -0.3029 \\
2 & 0.8357 & -0.5204 & 1 & -0.157 & 0.5155 \\
3 & $\mid$ & 0.1554 & 0.7713 & -0.157 & 1 & 0.1556 \\
4 & 0.5407 & -0.3029 & 0.5155 & 0.1556 & 1 \\
5 & -0.01515 & 0.5391 & -0.4107 & 0.477 & -0.2196 \\
6 & -0.1353 & -0.01806 & -0.05153 & -0.07615 & -0.0155 \\
7 & 0.1329 & -0.1756 & 0.1565 & -0.04219 & 0.141 \\
8 & 0.06097 & -0.09621 & 0.08569 & -0.03675 & 0.1387 \\
9 & -0.1682 & 0.13 & -0.1347 & 0.01787 & -0.07357 \\
10 & $\mid$ & 0.5228 & 0.1503 & 0.1681 & 0.4585 & 0.1887 \\
11 & -0.5087 & 0.6745 & -0.667 & 0.2112 & -0.7863 \\
12 & -0.2868 & 0.1802 & -0.2207 & -0.05966 & -0.1164 \\
13 & $\mid$ & -0.04772 & -0.03172 & 0.04465 & -0.06311 & 0.1999 \\
14 & -0.05465 & 0.06647 & -0.05539 & -0.03166 & -0.04545 \\
15 & -0.08901 & 0.02413 & -0.03172 & -0.04343 & 0.05924 \\
16 & 0.0579 & 0.02674 & 0.009089 & 0.06522 & -0.05452 \\
17 & -0.1131 & 0.2479 & -0.1978 & 0.07684 & -0.2322 \\
18 & $\mid$ & -0.276 & 0.1633 & -0.2082 & -0.02957 & -0.1589 \\
19 & 0.04161 & -0.1691 & 0.1234 & -0.106 & 0.1646 \\
20 & -0.03848 & -0.009926 & -0.01021 & -0.01175 & -0.004637 \\
21 & $\mid$ & 0.0599 & -0.01329 & 0.03247 & 0.023 & 0.02173 \\
22 & $\mid$ & 0.09854 & -0.2499 & 0.1966 & -0.06721 & 0.2539 \\
23 & -0.1042 & -0.04156 & -0.01684 & -0.08125 & 0.06925 \\
24 & -0.2312 & 0.1911 & -0.2067 & 0.01687 & -0.2082 \\
25 & 0.003918 & -0.07692 & 0.05523 & -0.08746 & 0.1034 \\
26 & $\mid$ & -0.169 & 0.05698 & -0.09764 & -0.03304 & -0.06963 \\
27 & 0.03561 & -0.003513 & 0.01682 & 0.00415 & 0.002918 \\
28 & -0.2836 & 0.1486 & -0.2049 & -0.06843 & -0.1226 \\
29 & -0.1598 & 0.2909 & -0.23 & 0.1126 & -0.2131
\end{tabular}

\begin{tabular}{|c|c|c|c|c|c|}
\hline I & 51 & 61 & 7 & 81 & 9 \\
\hline 01 & -0.01515 & -0.1353 & 0.1329 & 0.06097 & -0.1682 \\
\hline 11 & 0.5391 & -0.01806 & -0.1756 & -0.09621 & 0.13 \\
\hline 21 & -0.4107 & -0.05153 & 0.1565 & 0.08569 & -0.1347 \\
\hline 31 & 0.477 & -0.07615 & -0.04219 & -0.03675 & 0.01787 \\
\hline 41 & -0.2196 & -0.0155 & 0.141 & 0.1387 & -0.07357 \\
\hline 51 & 1 & -0.09946 & -0.02705 & -0.02609 & -0.09017 \\
\hline 61 & -0.09946 & 1 & -0.0276 & -0.02194 & 0.02558 \\
\hline 71 & -0.02705 & -0.0276 & 1 & 0.0307 & 0.1195 \\
\hline
\end{tabular}




\begin{tabular}{r|rrrrr}
8 & -0.02609 & -0.02194 & 0.0307 & 1 & -0.06202 \\
9 & -0.09017 & 0.02558 & 0.1195 & -0.06202 & 1 \\
10 & 0.6832 & -0.2192 & 0.1577 & 0.09087 & -0.2114 \\
11 & 0.3494 & -0.1027 & -0.1679 & -0.227 & 0.183 \\
12 & -0.1473 & -0.03959 & -0.1173 & 0.3026 & 0.04804 \\
13 & -0.3004 & 0.02347 & 0.1371 & -0.1473 & 0.3923 \\
14 & -0.02874 & -0.03721 & -0.04732 & 0.09571 & -0.08144 \\
15 & -0.1831 & -0.03337 & 0.1177 & -0.1021 & 0.3342 \\
16 & 0.1928 & -0.02104 & 0.03927 & 0.1398 & -0.13 \\
17 & 0.1028 & -0.06639 & 0.03605 & 0.0299 & -0.0283 \\
18 & -0.1826 & -0.01285 & 0.0163 & 0.02245 & 0.3509 \\
19 & -0.1606 & 0.0419 & -0.01201 & -0.1588 & 0.01283 \\
20 & -0.03194 & 0.01625 & 0.03815 & 0.04781 & 0.05558 \\
21 & 0.05951 & 0.03758 & -0.04348 & -0.05002 & -0.1632 \\
22 & -0.1419 & 0.05789 & 0.05739 & -0.06812 & 0.2061 \\
23 & -0.2597 & -0.01566 & 0.09683 & -0.03585 & 0.2204 \\
24 & -0.05096 & -0.04939 & 0.02854 & 0.1133 & 0.1478 \\
25 & -0.1869 & 0.06194 & 0.02688 & -0.01812 & -0.001243 \\
26 & -0.0993 & -0.03873 & 0.09389 & 0.004726 & 0.3514 \\
27 & 0.04253 & 0.03821 & 0.003612 & 0.05381 & -0.1036 \\
28 & -0.2209 & -0.07249 & -0.1629 & -0.2181 & 0.07613 \\
29 & 0.002023 & -0.1811 & 0.06474 & -0.1663 & 0.2833
\end{tabular}

\begin{tabular}{|c|c|c|c|c|c|}
\hline I & $10 \quad$ & 111 & 12 & 13 & 14 \\
\hline 01 & 0.5228 & -0.5087 & -0.2868 & -0.04772 & -0.05465 \\
\hline 11 & 0.1503 & 0.6745 & 0.1802 & -0.03172 & 0.06647 \\
\hline 21 & 0.1681 & -0.667 & -0.2207 & 0.04465 & -0.05539 \\
\hline 31 & 0.4585 & 0.2112 & -0.05966 & -0.06311 & -0.03166 \\
\hline 41 & 0.1887 & -0.7863 & -0.1164 & 0.1999 & -0.04545 \\
\hline 51 & 0.6832 & 0.3494 & -0.1473 & -0.3004 & -0.02874 \\
\hline 61 & -0.2192 & -0.1027 & -0.03959 & 0.02347 & -0.03721 \\
\hline 71 & 0.1577 & -0.1679 & -0.1173 & 0.1371 & -0.04732 \\
\hline 81 & 0.09087 & -0.227 & 0.3026 & -0.1473 & 0.09571 \\
\hline 91 & -0.2114 & 0.183 & 0.04804 & 0.3923 & -0.08144 \\
\hline 101 & 1 & -0.1295 & -0.2681 & -0.3357 & -0.08065 \\
\hline $11 \mid$ & -0.1295 & 1 & 0.1741 & -0.007278 & 0.0479 \\
\hline $12 \mid$ & -0.2681 & 0.1741 & 1 & -0.04145 & 0.3547 \\
\hline 13 I & -0.3357 & -0.007278 & -0.04145 & 1 & 0.01535 \\
\hline 14 I & -0.08065 & 0.0479 & 0.3547 & 0.01535 & 1 \\
\hline 15 I & -0.2654 & 0.0735 & 0.04638 & 0.5447 & 0.01657 \\
\hline 161 & 0.1703 & -0.099 & 0.1921 & -0.2783 & 0.1964 \\
\hline 17 I & -0.04375 & 0.2739 & 0.2776 & 0.08383 & 0.462 \\
\hline 181 & -0.2429 & 0.2963 & 0.2305 & 0.2196 & -0.1554 \\
\hline 19 | & -0.1705 & -0.1922 & -0.1031 & 0.1459 & 0.2057 \\
\hline
\end{tabular}




\begin{tabular}{r|rrrrr}
20 & -0.03269 & -0.0337 & 0.0857 & -0.05826 & 0.01137 \\
21 & 0.03621 & -0.07938 & 0.02176 & -0.02492 & 0.09802 \\
22 & 0.03915 & -0.2518 & -0.3176 & 0.01122 & -0.4528 \\
23 & -0.2315 & 0.1017 & -0.1534 & 0.3338 & -0.2699 \\
24 & -0.1161 & 0.2543 & 0.2904 & 0.06046 & -0.04922 \\
25 & -0.1877 & -0.08817 & -0.07504 & 0.2281 & 0.144 \\
26 & -0.1622 & 0.1159 & 0.1654 & 0.122 & 0.08473 \\
27 & 0.01618 & -0.07243 & 0.02566 & -0.08642 & 0.1187 \\
28 & -0.6089 & 0.3085 & 0.0226 & 0.3581 & 0.007737 \\
29 & 0.01449 & 0.5437 & 0.07951 & 0.2759 & -0.07119
\end{tabular}

\begin{tabular}{|c|c|c|c|c|c|}
\hline I & 15 & 16 & 17 & 18 & 19 \\
\hline 01 & -0.08901 & 0.0579 & -0.1131 & -0.276 & 0.04161 \\
\hline 11 & 0.02413 & 0.02674 & 0.2479 & 0.1633 & -0.1691 \\
\hline 21 & -0.03172 & 0.009089 & -0.1978 & -0.2082 & 0.1234 \\
\hline 31 & -0.04343 & 0.06522 & 0.07684 & -0.02957 & -0.106 \\
\hline 41 & 0.05924 & -0.05452 & -0.2322 & -0.1589 & 0.1646 \\
\hline 51 & -0.1831 & 0.1928 & 0.1028 & -0.1826 & -0.1606 \\
\hline 61 & -0.03337 & -0.02104 & -0.06639 & -0.01285 & 0.0419 \\
\hline 71 & 0.1177 & 0.03927 & 0.03605 & 0.0163 & -0.01201 \\
\hline 81 & -0.1021 & 0.1398 & 0.0299 & 0.02245 & -0.1588 \\
\hline 91 & 0.3342 & -0.13 & -0.0283 & 0.3509 & 0.01283 \\
\hline $10 \mid$ & -0.2654 & 0.1703 & -0.04375 & -0.2429 & -0.1705 \\
\hline $11 \mid$ & 0.0735 & -0.099 & 0.2739 & 0.2963 & -0.1922 \\
\hline $12 \mid$ & 0.04638 & 0.1921 & 0.2776 & 0.2305 & -0.1031 \\
\hline 131 & 0.5447 & -0.2783 & 0.08383 & 0.2196 & 0.1459 \\
\hline $14 \mid$ & 0.01657 & 0.1964 & 0.462 & -0.1554 & 0.2057 \\
\hline $15 \mid$ & 1 & -0.128 & 0.1323 & 0.1397 & 0.1257 \\
\hline $16 \mid$ & -0.128 & 1 & 0.1934 & 0.1906 & 0.3401 \\
\hline 17 I & 0.1323 & 0.1934 & 1 & -0.2139 & 0.4608 \\
\hline $18 \mid$ & 0.1397 & 0.1906 & -0.2139 & 1 & -0.1395 \\
\hline 19 । & 0.1257 & 0.3401 & 0.4608 & -0.1395 & 1 \\
\hline 201 & 0.01926 & 0.6767 & 0.1311 & 0.4478 & 0.4787 \\
\hline $21 \mid$ & -0.1768 & 0.2284 & 0.5628 & -0.3661 & 0.6338 \\
\hline 22 । & -0.1128 & -0.1173 & -0.7419 & 0.4094 & -0.09901 \\
\hline 231 & 0.2586 & -0.7061 & -0.1659 & 0.2328 & -0.3636 \\
\hline $24 \mid$ & -0.05375 & 0.3297 & -0.1477 & 0.7167 & -0.3582 \\
\hline 25 । & 0.06645 & 0.1589 & 0.4391 & 0.1379 & 0.6617 \\
\hline 261 & 0.292 & 0.2702 & -0.2218 & 0.5906 & -0.1153 \\
\hline $27 \mid$ & 0.03328 & 0.4093 & 0.4475 & -0.1198 & 0.612 \\
\hline 281 & 0.2922 & -0.255 & 0.05661 & 0.05732 & 0.1923 \\
\hline 291 & 0.1926 & -0.3731 & 0.06554 & 0.4546 & -0.1347 \\
\hline
\end{tabular}




\begin{tabular}{|c|c|c|c|c|c|c|}
\hline & I & 20 & $21 \quad \mid$ & 22 & 23 & 24 \\
\hline 0 & & -0.03848 & 0.0599 & 0.09854 & -0.1042 & -0.2312 \\
\hline 1 & I & -0.009926 & -0.01329 & -0.2499 & -0.04156 & 0.1911 \\
\hline 2 & I & -0.01021 & 0.03247 & 0.1966 & -0.01684 & -0.2067 \\
\hline 3 & I & -0.01175 & 0.023 & -0.06721 & -0.08125 & 0.01687 \\
\hline 4 & I & -0.004637 & 0.02173 & 0.2539 & 0.06925 & -0.2082 \\
\hline 5 & I & -0.03194 & 0.05951 & -0.1419 & -0.2597 & -0.05096 \\
\hline 6 & I & 0.01625 & 0.03758 & 0.05789 & -0.01566 & -0.04939 \\
\hline 7 & | & 0.03815 & -0.04348 & 0.05739 & 0.09683 & 0.02854 \\
\hline 8 & I & 0.04781 & -0.05002 & -0.06812 & -0.03585 & 0.1133 \\
\hline 9 & I & 0.05558 & -0.1632 & 0.2061 & 0.2204 & 0.1478 \\
\hline 10 & I & -0.03269 & 0.03621 & 0.03915 & -0.2315 & -0.1161 \\
\hline 11 & I & -0.0337 & -0.07938 & -0.2518 & 0.1017 & 0.2543 \\
\hline 12 & I & 0.0857 & 0.02176 & -0.3176 & -0.1534 & 0.2904 \\
\hline 13 & I & -0.05826 & -0.02492 & 0.01122 & 0.3338 & 0.06046 \\
\hline 14 & I & 0.01137 & 0.09802 & -0.4528 & -0.2699 & -0.04922 \\
\hline 15 & I & 0.01926 & -0.1768 & -0.1128 & 0.2586 & -0.05375 \\
\hline 16 & I & 0.6767 & 0.2284 & -0.1173 & -0.7061 & 0.3297 \\
\hline 17 & I & 0.1311 & 0.5628 & -0.7419 & -0.1659 & -0.1477 \\
\hline 18 & I & 0.4478 & -0.3661 & 0.4094 & 0.2328 & 0.7167 \\
\hline 19 & | & 0.4787 & 0.6338 & -0.09901 & -0.3636 & -0.3582 \\
\hline 20 & I & 1 & 0.2223 & 0.2547 & -0.3167 & 0.4323 \\
\hline 21 & I & 0.2223 & 1 & -0.3278 & -0.4725 & -0.266 \\
\hline 22 & I & 0.2547 & -0.3278 & 1 & 0.2861 & 0.3061 \\
\hline 23 & I & -0.3167 & -0.4725 & 0.2861 & 1 & 0.07072 \\
\hline 24 & I & 0.4323 & -0.266 & 0.3061 & 0.07072 & 1 \\
\hline 25 & I & 0.3274 & 0.5468 & -0.1752 & -0.2389 & -0.1284 \\
\hline 26 & I & 0.1828 & -0.6175 & 0.3294 & 0.1611 & 0.4867 \\
\hline 27 & | & 0.6436 & 0.4257 & -0.1942 & -0.4366 & -0.1042 \\
\hline 28 & I & -0.07876 & -0.008576 & -0.09723 & 0.2514 & -0.09644 \\
\hline 29 & I & -0.035 & -0.1932 & 0.1077 & 0.4378 & 0.2305 \\
\hline
\end{tabular}

\begin{tabular}{|c|c|c|c|c|c|}
\hline I & $25 \quad \mid$ & $26 \quad$ & 27 & 28 & 29 \\
\hline 01 & 0.003918 & -0.169 & 0.03561 & -0.2836 & -0.1598 \\
\hline 11 & -0.07692 & 0.05698 & -0.003513 & 0.1486 & 0.2909 \\
\hline 21 & 0.05523 & -0.09764 & 0.01682 & -0.2049 & -0.23 \\
\hline 31 & -0.08746 & -0.03304 & 0.00415 & -0.06843 & 0.1126 \\
\hline 41 & 0.1034 & -0.06963 & 0.002918 & -0.1226 & -0.2131 \\
\hline 51 & -0.1869 & -0.0993 & 0.04253 & -0.2209 & 0.002023 \\
\hline 61 & 0.06194 & -0.03873 & 0.03821 & -0.07249 & -0.1811 \\
\hline 71 & 0.02688 & 0.09389 & 0.003612 & -0.1629 & 0.06474 \\
\hline 81 & -0.01812 & 0.004726 & 0.05381 & -0.2181 & -0.1663 \\
\hline 91 & -0.001243 & 0.3514 & -0.1036 & 0.07613 & 0.2833 \\
\hline
\end{tabular}




\begin{tabular}{|c|c|c|c|c|c|}
\hline 101 & -0.1877 & -0.1622 & 0.01618 & -0.6089 & 0.01449 \\
\hline 111 & -0.08817 & 0.1159 & -0.07243 & 0.3085 & 0.5437 \\
\hline 12 I & -0.07504 & 0.1654 & 0.02566 & 0.0226 & 0.07951 \\
\hline 131 & 0.2281 & 0.122 & -0.08642 & 0.3581 & 0.2759 \\
\hline 14 I & 0.144 & 0.08473 & 0.1187 & 0.007737 & -0.07119 \\
\hline 151 & 0.06645 & 0.292 & 0.03328 & 0.2922 & 0.1926 \\
\hline 161 & 0.1589 & 0.2702 & 0.4093 & -0.255 & -0.3731 \\
\hline 17 I & 0.4391 & -0.2218 & 0.4475 & 0.05661 & 0.06554 \\
\hline 181 & 0.1379 & 0.5906 & -0.1198 & 0.05732 & 0.4546 \\
\hline 19 | & 0.6617 & -0.1153 & 0.612 & 0.1923 & -0.1347 \\
\hline 20 & 0.3274 & 0.1828 & 0.6436 & -0.07876 & -0.035 \\
\hline 211 & 0.5468 & -0.6175 & 0.4257 & -0.008576 & -0.1932 \\
\hline 221 & -0.1752 & 0.3294 & -0.1942 & -0.09723 & 0.1077 \\
\hline 231 & -0.2389 & 0.1611 & -0.4366 & 0.2514 & 0.4378 \\
\hline 241 & -0.1284 & 0.4867 & -0.1042 & -0.09644 & 0.2305 \\
\hline 251 & 1 & -0.2248 & 0.5234 & 0.1432 & -0.03996 \\
\hline 261 & -0.2248 & 1 & -0.2168 & 0.02286 & 0.1774 \\
\hline 27 I & 0.5234 & -0.2168 & 1 & -0.01875 & -0.1923 \\
\hline 281 & 0.1432 & 0.02286 & -0.01875 & 1 & 0.1542 \\
\hline 291 & -0.03996 & 0.1774 & -0.1923 & 0.1542 & 1 \\
\hline
\end{tabular}

\subsection{Fit with CLEO parametrization}

In this section we give the results of an alternative parametrization, where we fit the data with only $\rho(770)$ and a nonresonant term, as done by the CLEO collaboration [41]. The results of this fit are shown in Table 5.3. The Dalitz plot and invariant mass-squared projections for this fit are shown in are shown in Fig. 5.4. As can be easily seen, this parametrization does not reproduce the features observed in our data.

Table 5.3: Result of the fit to the $D^{0} \rightarrow \pi^{-} \pi^{+} \pi^{0}$ Dalitz plot with only $\rho(770)$ states and a nonresonant term. The errors are statistical only. The $\chi^{2} / \nu$ for the fit is 2.82 for $\nu=574$.

\begin{tabular}{l|r|r|r}
\hline \hline State & Amplitude $a_{r}$ & Phase $\phi_{r}$ & Fraction $f_{r}(\%)$ \\
\hline$\rho^{+}(770)$ & 1 & 0 & 71.7 \\
\hline$\rho^{0}(770)$ & $0.594 \pm 0.004$ & $11.1 \pm 0.5$ & $26.2 \pm 0.4$ \\
\hline$\rho^{-}(770)$ & $0.680 \pm 0.004$ & $-4.0 \pm 0.5$ & $33.1 \pm 0.4$ \\
\hline Nonres & $1.21 \pm 0.02$ & $-114.5 \pm 1.2$ & $4.1 \pm 0.1$ \\
\hline \hline
\end{tabular}



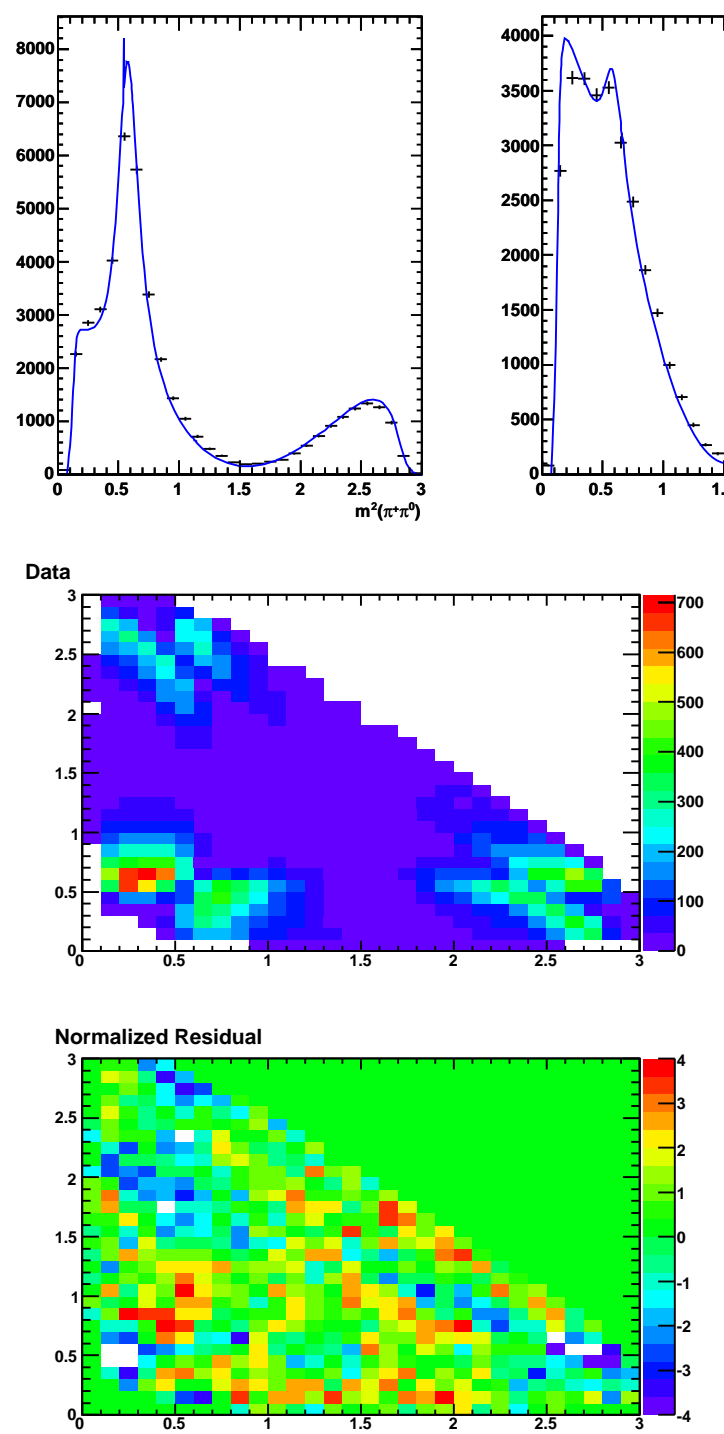
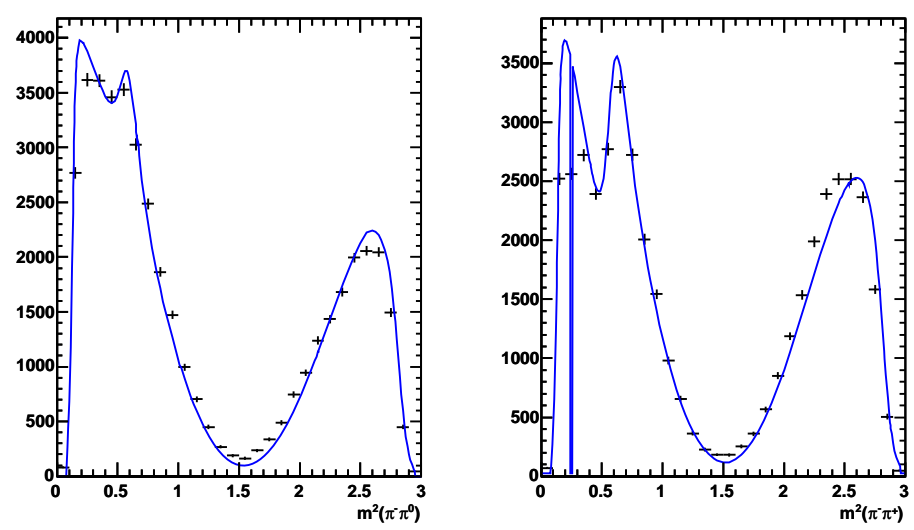

Fit with CLEO Model
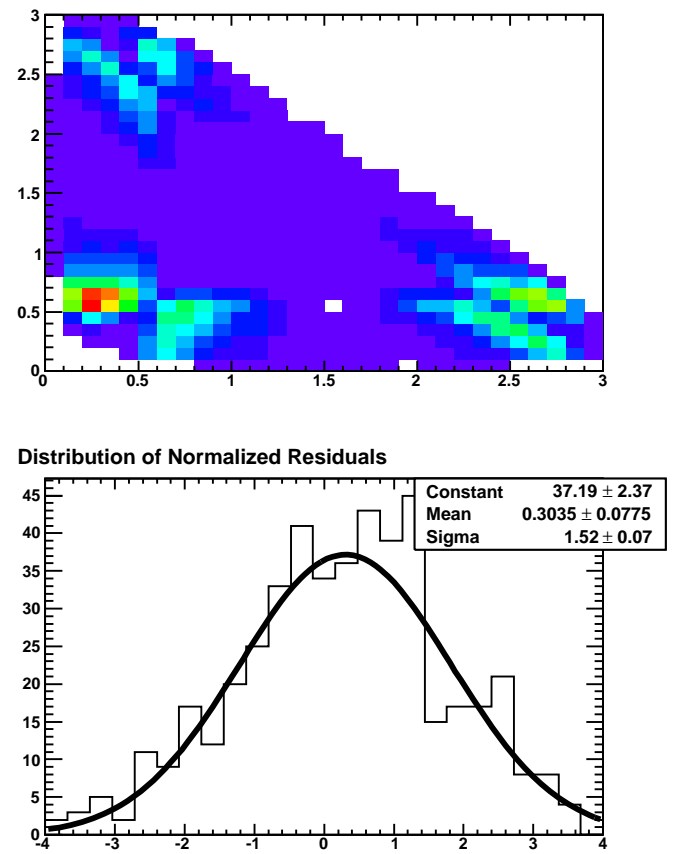

Figure 5.4: Invariant mass projections (top row), Dalitz plot (second row), and normalized residuals (bottom row) for the fit with only $\rho(770)$ and a nonresonant term. 


\subsection{Effort to include $p$-wave nonresonant terms}

When we fit the data with nonresonant p-wave components, the fit fractions of these components are of order 10. To understand why this is so, we study a PDF with only these components, and with the parameters obtained in the data. These parameters are given in Table 5.4. Also given in the table are the fit fractions for the three nonresonant p-wave components.

It is evident from the magnitude of the fit fractions that with their amplitudes and phases nearly identical, the three nonresonant p-wave amplitudes experience strong destructive interference, so that they cancel down to about one part in 500 . We note that the amplitudes and fractions for the three components are consistent with being identical. When setting them to be truly identical, we find that their fit fractions are 11700.

Furthermore, the cross-terms of these states with the charged rho's also have large destructive interference, as evidenced by their $\mathcal{O}(10)$ fit fractions on the data, and validated in toy fits that contain only nonresonant p-wave and rho terms.

Given the hugely destructive interference, it is difficult to conclude that the nonresonant $\mathrm{p}$-wave contributions in the data actually have a physical origin. It seems quite likely that the parameters that the fit finds for these terms are such that they help to improve the goodness of the fit only due to the greater flexibility that they provide, without actually corresponding to true physical states.

\begin{tabular}{lccc}
\hline \hline P-wave pair & Amplitude & Phase & Fit fraction \\
\hline$\pi^{0} \pi^{+}$ & $15.6 \pm 1.7$ & $-51.4 \pm 7.5$ & 483 \\
$\pi^{-} \pi^{0}$ & $16.3 \pm 1.7$ & $-51.8 \pm 7.1$ & 518 \\
$\pi^{+} \pi^{-}$ & $15.8 \pm 1.7$ & $-50.7 \pm 7.5$ & 475 \\
\hline \hline
\end{tabular}

Table 5.4: Parameters of the nonresonant p-wave found in the data fit, and the corresponding fit fractions (not in percent!) of these components for a PDF that contains only these components with the parameters shown.

\subsection{Description of angular moments}

Since the decay $D^{0} \rightarrow \pi^{-} \pi^{+} \pi^{0}$ has three spinless particles in the final state, the Dalitz plots in Fig. 5.3 uniquely represents the kinematics of the final state. The angular distributions provide information on the detailed event-density variations in various regions of the phase space in a different form. We define the helicity angle $\theta_{H}$ for decays $D^{0} \rightarrow(r \rightarrow A B) C$ as the angle between the momentum of $A$ in the $A B$ rest frame and the momentum of $A B$ in $D^{0}$ rest frame. The moments of the cosine of the helicity angle, $Y_{l}^{0}\left(\theta_{H}\right)$ are defined as the efficiency-corrected invariant mass distributions of events when weighted by spherical harmonic functions

$$
Y_{l}^{0}\left(\theta_{H}\right)=\sqrt{\frac{1}{2 \pi}} P_{l}\left(\cos \theta_{H}\right)
$$


where $P_{l}$ are Legendre polynomials of order $l$ :

$$
\int_{-1}^{1} P_{l}(x) P_{n}(x) d x=\delta_{l n} .
$$

Figure 5.5 shows the distribution of $P_{l}(m)$ for events corresponding to the decay $D^{0} / \bar{D}^{0} \rightarrow \pi^{-} \pi^{+} \pi^{0}$ for the channels $\pi^{+} \pi^{0}$ and $\pi^{+} \pi^{-}$. The moments in the $\pi^{-} \pi^{0}$ channel have distributions similar to those of $\pi^{+} \pi^{0}$.

These angular moments have an obvious physical significance. Since spherical harmonic functions are the eigen functions of the angular momentum, the Dalitz plot of a three-body decay can be represented by the sum of an infinite number of spherical harmonic moments in any two-body channel. In a region of the Dalitz plot where $S$ - and $P$-waves in a single channel dominate, their amplitudes are given by the following Legendre polynomial moments,

$$
P_{0}=\frac{|S|^{2}+|P|^{2}}{\sqrt{2}}, P_{1}=\sqrt{2}|S||P| \cos \theta_{S P}, P_{2}=\sqrt{\frac{2}{5}}|P|^{2},
$$

where $|S|$ and $|P|$ are, respectively, the magnitudes of the $S$ - and $P$-wave amplitudes, and $\theta_{S P}=\theta_{S}-\theta_{P}$ is the relative phase between them. It is worth noting that this partial-wave analysis is valid, in the absence of higher spin states, only if no interference occurs from the crossing channels. In the presence of substantial interference from crossing channels, it is not possible to decompose the Dalitz plot amplitude into partial waves (i.e., $S, P, D$ waves etc.). Because of the large interference from crossing channels in the decay $D^{0} \rightarrow \pi^{-} \pi^{+} \pi^{0}$, the model-independent partial-wave analysis using this method is not possible in the present case (unlike in the case of $D^{0} \rightarrow K^{-} K^{+} \pi^{0}$, as described in Chapter 4).

\subsection{Systematic uncertainties}

We determine the experimental and model systematic uncertainties separately and add them in quadrature to obtain the total systematic uncertainty. The only significant model dependent uncertainty comes from the large uncertainties in the masses and widths of the $\sigma$ and $\rho(1700)$ resonances. We vary their masses and widths and assign the larger of the variation in the $\sigma$ and $\rho(1700)$ fit parameter values with respect to the nominal fit parameters as model systematics. The systematic uncertainty due to reconstruction efficiency parametrization is estimated by comparing results of the nominal fit with the fit performed with uniform efficiency. Similarly, we study the systematic uncertainty due to different particle identification efficiencies in data and simulation by comparing the nominal fit results with the one performed without applying correction for this difference. We estimate the uncertainty due to the background shape by using simulation, instead of the $m_{D^{0}}$ sideband, to model the distribution of background events. We assign the maximum deviation in the observed quantities (amplitudes, phases, and fit fractions) with respect to the nominal fit as the systematic uncertainties. 



Figure 5.5: The mass dependence of the spherical harmonic moments of $\cos \theta_{H}$ after efficiency corrections and background subtraction: $\pi^{+} \pi^{0}$ (top) and $\pi^{-} \pi^{+}$(bottom) channels of the $D^{0} / \bar{D}^{0} \rightarrow \pi^{-} \pi^{+} \pi^{0}$ decay. The circles with error-bars are data points and the curves (red) are derived from the fit parametrization of Table 5.1. For the sake of visibility, we do not show error bars on the curves. 


\subsection{Strong-phase difference and suppression factor}

Neglecting $C P$ violation, the strong phase difference, $\delta_{D}$, between the $\bar{D}^{0}$ and $D^{0}$ decays to $\rho(770)^{+} \pi^{-}$state and their amplitude ratio, $r_{D}$, are given by

$$
r_{D} e^{i \delta_{D}}=\frac{a_{D^{0} \rightarrow \rho^{-} \pi^{+}}}{a_{D^{0} \rightarrow \rho^{+} \pi^{-}}} e^{i\left(\delta_{\rho^{-} \pi^{+}}-\delta_{\rho^{+} \pi^{-}}\right)} .
$$

Using the results of Table 5.1, we find $\delta_{D}=-2.0^{\circ} \pm 0.6^{\circ}$ (stat) $\pm 0.6^{\circ}$ (syst) and $r_{D}$ $=0.714 \pm 0.008$ (stat) \pm 0.003 (syst). These results are consistent with the previous measurements [41], $\delta_{D}=-4^{\circ} \pm 3^{\circ}$ (stat) $\pm 4^{\circ}$ (syst) and $r_{D}=0.65 \pm 0.03$ (stat) \pm 0.04 (syst).

\subsection{Summary}

In conclusion, we have studied the amplitude structure of the decay $D^{0} \rightarrow \pi^{-} \pi^{+} \pi^{0}$, and measured $\delta_{D}$ and $r_{D}$. We find significant contributions from the three $\rho(770)$ states. The decay $D^{0} \rightarrow \rho(770)^{+} \pi^{-}$dominates over $D^{0} \rightarrow \rho(770)^{-} \pi^{+}$. This suggests that, in tree-level diagrams, the form factor for $D^{0}$ coupling to $\rho^{-}$is suppressed compared to the corresponding $\pi^{-}$coupling.

The measured fit fractions for the observed states do not agree well with a phenomenological prediction [36] based on a large SU(3) symmetry breaking. It appears from the Dalitz plot distribution of Fig. 5.3 and the results reported in Table 5.1, that the decay $D^{0} \rightarrow \pi^{-} \pi^{+} \pi^{0}$ is marked by three destructively interfering $\rho \pi$ amplitudes (with different radial excitations of the $\rho$ states), suggesting an $I=0, \Delta I=1 / 2$ dominated final state [37]. 


\section{Chapter 6}

\section{Measurement of $\gamma$ Using $B^{ \pm} \rightarrow D_{\pi^{+} \pi^{-} \pi^{0}} K^{ \pm}$}

We report [25] the results of a $C P$ violation analysis of the decay $B^{ \pm} \rightarrow D_{\pi^{+} \pi^{-} \pi^{0}} K^{ \pm}$, where $D^{0} \rightarrow \pi^{-} \pi^{+} \pi^{0}$ indicates a neutral $D$ meson detected in the final state $\pi^{+} \pi^{-} \pi^{0}$, excluding $K_{S}^{0} \pi^{0}$. The analysis makes use of 324 million $e^{+} e^{-} \rightarrow B \bar{B}$ events recorded by the BABAR experiment at the PEP-II $e^{+} e^{-}$storage ring. By analyzing the $\pi^{+} \pi^{-} \pi^{0}$ Dalitz plot distribution and the $B^{ \pm} \rightarrow D_{\pi^{+} \pi^{-} \pi^{0}} K^{ \pm}$branching fraction and decay rate asymmetry, we calculate parameters related to the phase $\gamma$ of the CKM unitarity triangle. We also measure the magnitudes and phases of the components of the $D^{0} \rightarrow \pi^{+} \pi^{-} \pi^{0}$ decay amplitude. We observe $170 \pm 29 B^{ \pm} \rightarrow D_{\pi^{+} \pi^{-} \pi^{0}} K^{ \pm}$ events and calculate the branching fraction and decay rate asymmetry: $\mathcal{B}\left(B^{ \pm} \rightarrow\right.$ $\left.D_{\pi^{+} \pi^{-} \pi^{0}} K^{ \pm}\right)=(4.6 \pm 0.8 \pm 0.4) \times 10^{-6}, A\left(B^{ \pm} \rightarrow D_{\pi^{+} \pi^{-} \pi^{0}} K^{ \pm}\right)=-0.02 \pm 0.15 \pm 0.03$, the errors being statistical and systematic, respectively. We find the $C P$-violation parameters: $\rho_{-}=0.72 \pm 0.11 \pm 0.06, \theta_{-}=(173 \pm 42 \pm 19)^{\circ}, \rho_{+}=0.75 \pm 0.11 \pm 0.06$, $\theta_{+}=(147 \pm 23 \pm 13)^{\circ}$, where the first errors are statistical and the second are systematic. The parameters $\rho_{ \pm}, \theta_{ \pm}$are defined as $\rho_{ \pm} \equiv\left|z_{ \pm}-x_{0}\right|, \theta_{ \pm} \equiv \tan ^{-1}\left(\frac{\Im\left[z_{ \pm}\right]}{\Re\left[z_{ \pm}\right]-x_{0}}\right)$,

where $z_{ \pm} \equiv r_{B} e^{i(\delta \pm \gamma)}$ ( $\delta$ is a $C P$-even phase and $r_{B}$ is the ratio of the magnitudes of the $b \rightarrow u \bar{c} s$ and $b \rightarrow c \bar{u} s$ amplitudes), and $x_{0}=0.850$ is a coordinate transformation parameter. Using these results, we find the following one-standard-deviation constraints on the amplitude ratio and on the weak and strong phases: $0.06<r_{B}<0.78$, $-30^{\circ}<\gamma<76^{\circ},-27^{\circ}<\delta<78^{\circ}$.

To apply the results of the amplitude analysis of the decay $D^{0} \rightarrow \pi^{-} \pi^{+} \pi^{0}$ to the measurement of quantities related to the quark-mixing phase $\gamma$ (or $\phi_{3}$ ) using the decay chain $B^{ \pm} \rightarrow D_{\pi^{+} \pi^{-} \pi^{0}} K^{ \pm}$, I teamed up with three other BABAR colleagues from different institutions: Abi Soffer, Frank Winklmeier, and Jinlong Zhang. Because the decay $D^{0} \rightarrow \pi^{-} \pi^{+} \pi^{0}$ involves three particles in the final state, it proceeds via intermediate resonances which interfere with each other. I performed a detailed analysis of the quantum-mechanical amplitudes involved in these decays, as described in chapter 5 , in order to understand the relative contributions and underlying properties of the intermediate resonances. As a part of this team, I also helped in understanding the likelihood behavior of the fit for extracting $C P$ violation parameters related to phase $\gamma$, and contributed to the documentation of the analysis procedure. I am 
listed as a leading author of this analysis, Abi Soffer being the primary corresponding author.

\subsection{Introduction}

An important component of the program to study $C P$ violation is the measurement of the angle $\gamma=\arg \left(-V_{u d} V_{u b}^{*} / V_{c d} V_{c b}^{*}\right)$ of the unitarity triangle related to the CabibboKobayashi-Maskawa quark mixing matrix [2]. The decays $B \rightarrow D^{(*) 0} K^{(*)}$ can be used to measure $\gamma$ with essentially no hadronic uncertainties, exploiting interference between $b \rightarrow u \bar{c} s$ and $b \rightarrow c \bar{u} s$ decay amplitudes [4]. In one of the measurement methods [6], $\gamma$ is extracted by analyzing the $D$-decay Dalitz plot distribution in $B^{ \pm} \rightarrow D K^{ \pm}$with multi-body $D$ decays ${ }^{1}$. This method has only been used with the Cabibbo-favored (also has a small doubly Cabibbo-suppressed component) decay $D \rightarrow K_{S}^{0} \pi^{+} \pi^{-}[38,39]$, and Cabibbo-suppressed decays are expected to be similarly sensitive to $\gamma[40]$. We present here the first $C P$-violation study of $B^{ \pm} \rightarrow D K^{ \pm}$with a multibody, Cabibbo-suppressed $D$ decay, $D \rightarrow \pi^{+} \pi^{-} \pi^{0}$.

\subsection{Detector and dataset}

The data used in this analysis were collected with the BABAR detector at the PEP-II $e^{+} e^{-}$storage ring, and include $288 \mathrm{fb}^{-1}$ taken on the $\Upsilon(4 \mathrm{~S})$ resonance and $27 \mathrm{fb}^{-1}$ collected $40 \mathrm{MeV}$ below the resonance. Samples of simulated Monte Carlo (MC) events were analyzed with the same reconstruction and analysis procedures. These samples include an $e^{+} e^{-} \rightarrow B \bar{B}$ sample about five times larger than the data; a continuum $e^{+} e^{-} \rightarrow q \bar{q}$ sample, where $q$ is a $u, d, s$, or $c$ quark, with luminosity equivalent to the data; and a signal sample about 300 times larger than the data, with both phase space $D$ decays and decays generated according to the amplitudes measured by CLEO [41]. The BABAR detector and the methods used for particle reconstruction and identification are described in Ref. [12].

\subsection{Event selection}

We use event-shape variables [42] to suppress continuum background, and identify kaon and pion candidates using specific ionization and Cherenkov radiation. The invariant mass of $D$ candidates must satisfy $1830<M_{D}<1895 \mathrm{MeV} / \mathrm{c}^{2}$. We require $5272<m_{E S}<5300 \mathrm{MeV} / c^{2}$, where $m_{E S} \equiv \sqrt{E_{\mathrm{CM}}^{2} / 4-\left|\mathbf{p}_{\mathbf{B}}\right|^{2}}, E_{\mathrm{CM}}$ is the total $e^{+} e^{-}$center-of-mass $(\mathrm{CM})$ energy, and $\mathbf{p}_{\mathbf{B}}$ is the $B$ candidate CM momentum. Events must satisfy $-70<\Delta E<60 \mathrm{MeV}$, where $\Delta E=E_{B}-E_{\mathrm{CM}} / 2$ and $E_{B}$ is the $B$ candidate $\mathrm{CM}$ energy. We exclude the decay mode $D \rightarrow K_{S}^{0} \pi^{0}$, which is a previously studied $C P$ eigenstate not related to the method of Ref. [6], by rejecting candidates with $489<M\left(\pi^{+} \pi^{-}\right)<508 \mathrm{MeV} / c^{2}$ or for which the distance between the $\pi^{+} \pi^{-}$vertex and the $B^{-}$candidate decay vertex is more than $1.5 \mathrm{~cm}$.

\footnotetext{
${ }^{1}$ We use the symbol $D$ in this chapter to indicate any linear combination of a $D^{0}$ and a $\bar{D}^{0}$ meson state.
} 
We reject $B^{ \pm} \rightarrow D_{\pi^{+} \pi^{-} \pi^{0}} K^{ \pm}$candidates in which the $K^{ \pm} \pi^{\mp}$ invariant mass satisfies $1840<M\left(K^{ \pm} \pi^{\mp}\right)<1890 \mathrm{MeV} / c^{2}$, to suppress $B^{-} \rightarrow D_{K^{-} \pi^{+}}^{0} \rho^{-}$decays. We require $d>0.25$, where $d[42]$ is a neural net variable that separates signal candidates (which peak toward $d=1$ ) from those with a misreconstructed $D$ (peaking toward $d=0$ ). In events with multiple candidates ( $9 \%$ of the sample), we keep the candidate whose $m_{E S}$ value is closest to the nominal $B^{ \pm}$mass [20]. The final signal reconstruction efficiency is $\epsilon=11.4 \%$.

\subsection{Signal-background discrimination}

For each $B^{ \pm} \rightarrow D_{\pi^{+} \pi^{-} \pi^{0}} K^{ \pm}$candidate, we compute the neural net variable $q$ [42]. The $q$ distribution of $B \bar{B}$ events peaks toward $q=1$, while that of continuum peaks at $q=0$. For $\nu \in\{q, d\}$, we define the variables $\nu^{\prime} \equiv \tanh ^{-1}\left[\frac{\nu-\frac{1}{2}\left(\nu_{\max }+\nu_{\min }\right)}{\frac{1}{2}\left(\nu_{\max }+\nu_{\min }\right)}\right]$, where $q_{\max }=d_{\max }=1, q_{\min }=0.1$, and $d_{\min }=0.25$ are the allowed ranges for $q$ and $d$. The $\nu^{\prime}$ variables can be conveniently fit with Gaussians, as described later.

\subsection{Event types}

As in Ref. [42], we identify in the MC samples ten event types, one signal and nine different backgrounds. We list them here with the labels used to refer to them throughout the paper. $\boldsymbol{D} \boldsymbol{K}_{\text {sig: }} \quad B^{ \pm} \rightarrow D_{\pi^{+} \pi^{-} \pi^{0}} K^{ \pm}$events that are correctly reconstructed; these are the only events considered to be signal. $\boldsymbol{D} \boldsymbol{K}_{\mathbf{b g d}}$ : $B^{ \pm} \rightarrow D_{\pi^{+} \pi^{-} \pi^{0}} K^{ \pm}$events that are misreconstructed; that is, some of the particles used to form the final state do not originate from the $B^{ \pm} \rightarrow D_{\pi^{+} \pi^{-} \pi^{0}} K^{ \pm}$decay. $\boldsymbol{D} \boldsymbol{\pi}_{\boldsymbol{D}}\left(\boldsymbol{D} \pi_{\not D}\right): B^{-} \rightarrow D^{0} \pi^{-}, D^{0} \rightarrow \pi^{+} \pi^{-} \pi^{0}$ decays, where the decay $D^{0} \rightarrow \pi^{+} \pi^{-} \pi^{0}$ is correctly reconstructed (misreconstructed). $\boldsymbol{D} \boldsymbol{K} \boldsymbol{X}: B \rightarrow D^{(*)} K^{(*)-}$ events not containing the decay $D \rightarrow \pi^{+} \pi^{-} \pi^{0} . \boldsymbol{D} \boldsymbol{\pi} \boldsymbol{X}: B \rightarrow D^{(*)} \pi^{-}$and $B \rightarrow D^{(*)} \rho^{-}$decays, excluding $D \rightarrow \pi^{+} \pi^{-} \pi^{0}$. $\boldsymbol{B} \boldsymbol{B} \boldsymbol{C}_{\boldsymbol{D}}\left(\boldsymbol{B} \boldsymbol{B} \boldsymbol{C}_{\not D}\right)$ : all other $B \bar{B}$ events with a correctly reconstructed (misreconstructed) $D$ candidate. $\boldsymbol{q q}_{\boldsymbol{D}}\left(\boldsymbol{q q}_{\not \mathbf{D}}\right)$ : continuum $e^{+} e^{-} \rightarrow q \bar{q}$ events with a correctly reconstructed (misreconstructed) $D$ candidate.

\subsection{Analysis procedure}

The measurement of the $C P$ parameters proceeds in three steps, each involving an unbinned maximum likelihood fit. In step 1 (described in detail in chapter 5), we measure the complex Dalitz plot amplitude $\alpha\left(s_{+}, s_{-}\right)$for the decay $D^{0} \rightarrow \pi^{+} \pi^{-} \pi^{0}$, where $s_{ \pm}=m^{2}\left(\pi^{ \pm} \pi^{0}\right)$ are the squared invariant masses of the $\pi^{ \pm} \pi^{0}$ pairs. In step 2 , we extract the numbers of $B^{+}$and $B^{-}$signal events and background yields. We obtain the $C P$ parameters in step 3.

We parameterize $\alpha\left(s_{+}, s_{-}\right)$using the isobar model, $\alpha\left(s_{+}, s_{-}\right)=\left[a_{\mathrm{NR}} e^{i \phi_{\mathrm{NR}}}+\right.$ $\left.\sum_{r} a_{r} e^{i \phi_{r}} A_{r}\left(s_{+}, s_{-}\right)\right] / N_{\alpha}$, where the first term represents a nonresonant contribution, the sum is over all intermediate two-body resonances $r$, and $N_{\alpha}$ is such that $\int d s_{+} d s_{-}\left|\alpha\left(s_{+}, s_{-}\right)\right|^{2}=1$. The amplitude for the decay chain $D^{0} \rightarrow r C, r \rightarrow A B$ is $A_{r}\left(s_{+}, s_{-}\right)=F_{r} F_{s}\left(m_{r}^{2}-M_{A B}^{2}-i m_{r} \Gamma_{r}\left(M_{A B}\right)\right)^{-1}$, where $m_{r}$ is the peak mass of the resonance [20], $M_{A B}^{2}$ is the squared invariant mass of the $A B$ pair, $F_{r}$ is a 
spin-dependent form factor [43], and $\Gamma_{r}\left(M_{A B}\right)$ is the mass-dependent width for the resonance $r$ [43]. The spin factors $F_{s}$ are $F_{0}=m_{D}^{2}, F_{1}=M_{B C}^{2}-M_{A C}^{2}+\left(m_{D}^{2}-\right.$ $\left.m_{C}^{2}\right)\left(m_{A}^{2}-m_{B}^{2}\right) M_{A B}^{-2}$, and $F_{2}=\left(F_{1}^{2}-{ }_{3}^{1} \mu_{C D}^{2} \mu_{A B}^{2}\right) m_{D}^{-2}$, where $\mu_{j k}^{2} \equiv M_{A B}^{2}-2 m_{j}^{2}-$ $2 m_{k}^{2}+\left(m_{j}^{2}-m_{k}^{2}\right)^{2} M_{j k}^{-2}$, and $m_{i}$ is the mass of particle $i[20]$.

\subsection{Step 1: $D^{0}$ Dalitz plot fit using $D^{*}$ sample}

In step 1 , we determine the parameters $a_{\mathrm{NR}}, a_{r}, \phi_{\mathrm{NR}}$, and $\phi_{r}$ by fitting a large sample of $D^{0}$ and $\bar{D}^{0}$ mesons, flavor-tagged through their production in the decay $D^{*+} \rightarrow$ $D^{0} \pi^{+}$[18]. To select this sample, we require the CM momentum of the $D^{*}$ candidate to be greater than $2770 \mathrm{MeV} / c$, and $\left|M_{D^{*}}-M_{D}-145.4 \mathrm{MeV} / c^{2}\right|<0.6 \mathrm{MeV} / c^{2}$, where $M_{D^{*}}$ is the invariant mass of the $D^{*}$ candidate. The signal reconstruction procedure is described in detail in Chapter 3. The signal and background yields are obtained from a fit to the reconstructed $M_{D}$ distribution, modeling the signal as a Gaussian and the background as an exponential. The signal Gaussian peaks at $1863.7 \pm 0.4 \mathrm{MeV} / \mathrm{c}^{2}$ and has a width of $17.4 \pm 0.8 \mathrm{MeV} / \mathrm{c}^{2}$.

Of the $D^{0}$ candidates in the signal region $1848<M_{D}<1880 \mathrm{MeV} / c^{2}$, we obtain from the fit $N_{S}=44780 \pm 250$ signal and $N_{B}=830 \pm 70$ background events. To obtain the parameters of $\alpha\left(s_{ \pm}, s_{\mp}\right)$, we fit these candidates with the probability distribution function (PDF) $N_{S}\left|\alpha\left(s_{+}, s_{-}\right)\right|^{2} \epsilon\left(s_{+}, s_{-}\right)+N_{B}\left|f_{B}\left(s_{+}, s_{-}\right)\right|^{2}$, where the background $\mathrm{PDF} f_{B}\left(s_{+}, s_{-}\right)$is a binned distribution obtained from events in the sideband $1930<$ $M_{D}<1990 \mathrm{MeV} / c^{2}$, and $\epsilon\left(s_{+}, s_{-}\right)$is an efficiency function, parameterized as a twodimensional third-order polynomial determined from MC. To within the MC-signal statistical uncertainty, $\epsilon\left(s_{+}, s_{-}\right)=\epsilon\left(s_{-}, s_{+}\right)$. The region $M_{D}<1848 \mathrm{MeV} / c^{2}$, which contains $D^{0} \rightarrow K^{-} \pi^{+} \pi^{0}$ events that are absent from the signal region, is not used.

Table 6.1 summarizes the results of this fit, with systematic errors obtained by varying the masses and widths of the $\rho(1700)$ and $\sigma$ resonances, setting $F_{r}=1$, and varying $\epsilon\left(s_{+}, s_{-}\right)$to account for uncertainties in reconstruction and particle identification. The Dalitz plot distribution of the data is shown in Fig. 6.1(a-c). The distribution is marked by three destructively interfering $\rho \pi$ amplitudes, suggesting an $I=0$-dominated final state [37]. We refer to Chapter 4 for further details on the Dalitz plot analysis of the decay $D^{0} \rightarrow \pi^{-} \pi^{+} \pi^{0}$.

\subsection{Next Steps}

The fit for step $i \in\{2,3\}$ uses the PDF

$$
\mathcal{P}_{i}^{C}=\sum_{t} \frac{N_{t}}{2 \eta}\left(1-C A_{t}\right) \mathcal{P}_{i, t}^{(C)}\left(\xi_{i}\right) \div \int \mathcal{P}_{i, t}^{(C)}\left(\xi_{i}^{\prime}\right) d^{n_{i}} \xi_{i}^{\prime},
$$

where $\xi_{i}$ is the set of $n_{i}$ event variables $\xi_{1}=\left\{\Delta E, q^{\prime}, d^{\prime}\right\}, \xi_{2}=\left\{\Delta E, q^{\prime}, s_{-}, s_{+}\right\}, t$ corresponds to one of the ten event types listed above, $N_{t}=N_{t}^{+}+N_{t}^{-}$is the number of events of type $t, A_{t}=\left(N_{t}^{-}-N_{t}^{+}\right) / N_{t}$ is their charge asymmetry, $C= \pm 1$ is the electric charge of the $B$ candidate, and $\eta \equiv \sum_{t} N_{t}$. Using MC, we verify that the variables in each set $\xi_{i}$ are uncorrelated for each event type. Therefore, the PDFs 
Table 6.1: Result of the fit to the $D^{*+} \rightarrow D^{0} \pi^{+}$sample, showing the amplitudes ratio $R_{r} \equiv a_{r} / a_{\rho^{+}(770)}$, phase differences $\Delta \phi_{r} \equiv \phi_{r}-\phi_{\rho^{+}(770)}$, and fit fractions $f_{r} \equiv \int\left|a_{r} A_{r}\left(s_{+}, s_{-}\right)\right|^{2} d s_{-} d s_{+}$. The first (second) errors are statistical (systematic). We take the mass (width) of the $\sigma$ meson to be 400 (600) $\mathrm{MeV} / c^{2}$.

\begin{tabular}{l|r|r|r}
\hline \hline State & $R_{r}(\%)$ & $\Delta \phi_{r}\left(^{\circ}\right)$ & $f_{r}(\%)$ \\
\hline$\rho^{+}(770)$ & 100 & 0 & $67.8 \pm 0.0 \pm 0.6$ \\
$\rho^{0}(770)$ & $58.8 \pm 0.6 \pm 0.2$ & $16.2 \pm 0.6 \pm 0.4$ & $26.2 \pm 0.5 \pm 1.1$ \\
$\rho^{-}(770)$ & $71.4 \pm 0.8 \pm 0.3$ & $-2.0 \pm 0.6 \pm 0.6$ & $34.6 \pm 0.8 \pm 0.3$ \\
$\rho^{+}(1450)$ & $21 \pm 6 \pm 13$ & $-146 \pm 18 \pm 24$ & $0.11 \pm 0.07 \pm 0.12$ \\
$\rho^{0}(1450)$ & $33 \pm 6 \pm 4$ & $10 \pm 8 \pm 13$ & $0.30 \pm 0.11 \pm 0.07$ \\
$\rho^{-}(1450)$ & $82 \pm 5 \pm 4$ & $16 \pm 3 \pm 3$ & $1.79 \pm 0.22 \pm 0.12$ \\
$\rho^{+}(1700)$ & $225 \pm 18 \pm 14$ & $-17 \pm 2 \pm 3$ & $4.1 \pm 0.7 \pm 0.7$ \\
$\rho^{0}(1700)$ & $251 \pm 15 \pm 13$ & $-17 \pm 2 \pm 2$ & $5.0 \pm 0.6 \pm 1.0$ \\
$\rho^{-}(1700)$ & $200 \pm 11 \pm 7$ & $-50 \pm 3 \pm 3$ & $3.2 \pm 0.4 \pm 0.6$ \\
$f_{0}(980)$ & $1.50 \pm 0.12 \pm 0.17$ & $-59 \pm 5 \pm 4$ & $0.25 \pm 0.04 \pm 0.04$ \\
$f_{0}(1370)$ & $6.3 \pm 0.9 \pm 0.9$ & $156 \pm 9 \pm 6$ & $0.37 \pm 0.11 \pm 0.09$ \\
$f_{0}(1500)$ & $5.8 \pm 0.6 \pm 0.6$ & $12 \pm 9 \pm 4$ & $0.39 \pm 0.08 \pm 0.07$ \\
$f_{0}(1710)$ & $11.2 \pm 1.4 \pm 1.7$ & $51 \pm 8 \pm 7$ & $0.31 \pm 0.07 \pm 0.08$ \\
$f_{2}(1270)$ & $104 \pm 3 \pm 21$ & $-171 \pm 3 \pm 4$ & $1.32 \pm 0.08 \pm 0.10$ \\
$\sigma(400)$ & $6.9 \pm 0.6 \pm 1.2$ & $8 \pm 4 \pm 8$ & $0.82 \pm 0.10 \pm 0.10$ \\
Non-Res & $57 \pm 7 \pm 8$ & $-11 \pm 4 \pm 2$ & $0.84 \pm 0.21 \pm 0.12$ \\
\hline \hline
\end{tabular}

$\mathcal{P}_{i, t}^{(C)}$ are the products

$$
\begin{aligned}
\mathcal{P}_{2, t}\left(\Delta E, q^{\prime}, d^{\prime}\right) & =\mathcal{E}_{t}(\Delta E) \mathcal{Q}_{t}\left(q^{\prime}\right) \mathcal{C}_{t}\left(d^{\prime}\right) \\
\mathcal{P}_{3, t}^{C}\left(\Delta E, q^{\prime}, s_{+}, s_{-}\right) & =\mathcal{E}_{t}(\Delta E) \mathcal{Q}_{t}\left(q^{\prime}\right) \mathcal{D}_{t}^{\prime}{ }_{t}\left(s_{+}, s_{-}\right) .
\end{aligned}
$$

The parameters of the Dalitz plot PDF $\mathcal{D}^{\prime C} K_{\text {sig }}\left(s_{+}, s_{-}\right)$are obtained from the data as described below. Those of all other functions in Eq. (6.2) are obtained from the MC samples. The functions $\mathcal{E}_{t}(\Delta E)$ are parameterized as the sum of a Gaussian and a second-order polynomial. The PDFs $\mathcal{Q}_{t}\left(q^{\prime}\right)$ and $\mathcal{C}_{t}\left(d^{\prime}\right)$ are the sum of a Gaussian and an asymmetric Gaussian. The PDF parameters are different for each event type. Assuming no $C P$ violation in the background, we take $\mathcal{D}_{t}^{\prime+}\left(s_{+}, s_{-}\right)=\mathcal{D}_{t}^{\prime-}\left(s_{-}, s_{+}\right)$ and $A_{t}=0$ for $t \neq D K_{\text {sig. }}$. The functions $\mathcal{D}_{D \pi X}^{\prime C}\left(s_{+}, s_{-}\right)$and $\mathcal{D}_{D K_{\mathrm{bgd}}}^{\prime C}\left(s_{+}, s_{-}\right)$ are binned histograms obtained from the MC. For other event types, $\mathcal{D}_{t}^{\prime C}\left(s_{+}, s_{-}\right)=$ $\epsilon\left(s_{+}, s_{-}\right) \mathcal{D}_{t}^{C}\left(s_{+}, s_{-}\right)$, where the efficiency function $\epsilon\left(s_{+}, s_{-}\right)$has different parameters for well-reconstructed and misreconstructed $D$ candidates.

We define $z_{ \pm} \equiv r_{B} e^{i(\delta \pm \gamma)}$, where $\delta$ is a $C P$-even phase and $r_{B}$ is the ratio of the magnitudes of the $b \rightarrow u \bar{c} s$ and $b \rightarrow c \bar{u} s$ amplitudes. Ignoring negligible $D^{0}-\bar{D}^{0}$ mixing effects [44], the signal Dalitz PDF is

$$
\mathcal{D}_{D K_{\mathrm{sig}}}^{ \pm}\left(s_{+}, s_{-}\right)=\left|\alpha\left(s_{\mp}, s_{ \pm}\right)+z_{ \pm} \alpha\left(s_{ \pm}, s_{\mp}\right)\right|^{2} .
$$




\subsection{Signal yields and asymmetry}

In the step-2 fit, we extract the $B^{ \pm} \rightarrow D_{\pi^{+} \pi^{-} \pi^{0}} K^{ \pm}$signal yield and asymmetry, as well as some background yields, as described in Ref. [42]. From this fit we find $N_{D K_{\text {sig }}}=170 \pm 29$ signal events and a decay rate asymmetry $A_{D K_{\mathrm{sig}}}=-0.02 \pm 0.15$. Errors are statistical only.

\subsection{Likelihood function}

Only the complex parameters $z_{ \pm}$are free in the step-3 fit. This fit minimizes the function

$$
\mathcal{L}=-\sum_{e=1}^{N_{\mathrm{ev}}} \log \mathcal{P}_{3}^{C_{e}}\left(\xi_{3}^{e}\right)+\frac{1}{2} \chi^{2},
$$

where $N_{\mathrm{ev}}$ is the number of events in the data sample. The term $\chi^{2}=\sum_{u, v=1}^{2} X_{u} V_{u v}^{-1} X_{v}$ increases the sensitivity of the fit by using the results of the step- 2 fit via

$$
\begin{aligned}
& X_{1}=N_{D K_{\mathrm{sig}}}-\left(n_{-}+n_{+}\right), \\
& X_{2}=A_{D K_{\mathrm{sig}}}-\left(n_{-}-n_{+}\right) /\left(n_{-}+n_{+}\right),
\end{aligned}
$$

where

$$
n_{ \pm}=N^{0} \frac{\int \mathcal{D}^{\prime}{ }_{D K_{\mathrm{sig}}}\left(s_{+}, s_{-}\right) d s_{+} d s_{-}}{\int\left|\alpha\left(s_{\mp}, s_{ \pm}\right)\right|^{2} \epsilon\left(s_{+}, s_{-}\right) d s_{+} d s_{-}}
$$

are the expected numbers of $B^{ \pm}$signal events. In Eq. (6.6), $N^{0}$ is the product of the number $N_{B^{+} B^{-}}$of charged $B^{+} B^{-}$pairs in the dataset, the branching fractions $\mathcal{B}\left(B^{-} \rightarrow D^{0} K^{-}\right)[20]$ and $\mathcal{B}\left(D^{0} \rightarrow \pi^{+} \pi^{-} \pi^{0}\right)$ [18], and the reconstruction efficiency $\epsilon$. The error matrix $V_{u v}$ is the sum of two components: the step-2 fit error matrix $V_{u v}^{\text {stat}}$, which is almost diagonal (the correlation coefficient is $-2.8 \%$ ), and the $N^{0}$ systematic error matrix $V_{u v}^{\text {syst }}$. Here $V_{12}^{\text {syst }}=V_{22}^{\text {syst }}=0$, and $V_{11}^{\text {syst }}=\sum_{c=1}^{4}\left(N^{0} \sigma_{c}^{\text {rel }}\right)^{2}$, where $\sigma_{c}^{\text {rel }}$ are the relative errors on the four components $N_{B^{+} B^{-}}(1.1 \%), \epsilon(3.3 \%)$, $\mathcal{B}\left(D \rightarrow \pi^{+} \pi^{-} \pi^{0}\right)(3.8 \%)[18]$, and $\mathcal{B}\left(B^{-} \rightarrow D^{0} K^{-}\right)(5.9 \%)[20]$.

\subsection{Polar coordinates}

We parameterize $z_{ \pm}$with the polar coordinates

$$
\rho_{ \pm} \equiv\left|z_{ \pm}-x_{0}\right|, \quad \theta_{ \pm} \equiv \tan ^{-1}\left(\frac{\Im\left[z_{ \pm}\right]}{\Re\left[z_{ \pm}\right]-x_{0}}\right)
$$

where $x_{0}$ is a coordinate transformation parameter,

$$
\begin{aligned}
x_{0} & \equiv-\int \Re\left[\alpha\left(s_{+}, s_{-}\right) \alpha^{*}\left(s_{-}, s_{+}\right)\right] \epsilon\left(s_{+}, s_{-}\right) d s_{+} d s_{-} \\
& =0.850 .
\end{aligned}
$$

This parameterization is optimal due to the polar symmetry of $n_{ \pm}=N^{0}\left(1+\rho_{ \pm}{ }^{2}-\right.$ $\left.x_{0}^{2}\right)$. Other parameterizations, such as $\left(r_{B}, \gamma, \delta\right)$ or $\left(\Re\left[z_{ \pm}\right], \Im\left[z_{ \pm}\right]\right)$, result in significant nonlinear correlations between the fit variables, which cannot be parameterized 
with an error matrix, and bias the fit result. The polar coordinates enable a significant improvement in sensitivity due to the $\chi^{2}$ term in Eq. (6.4), and are determined from parameterized simulation to be unbiased. The step-3 fit yields

$$
\begin{array}{ll}
\rho_{-}=0.72 \pm 0.11 \pm 0.04, & \theta_{-}=(173 \pm 42 \pm 2)^{\circ}, \\
\rho_{+}=0.75 \pm 0.11 \pm 0.04, & \theta_{+}=(147 \pm 23 \pm 1)^{\circ},
\end{array}
$$

where the first errors are statistical and the second are systematic, due only to $V_{11}^{\text {syst }}$. The largest correlation coefficient is $c_{\rho_{-} \rho_{+}}=14 \%$, originating from $V_{11}^{\text {syst }}$. All others are $1 \%$ or less. Contours of constant $\mathcal{L}$ values are shown in Fig. 6.1(d). Projections of the data and the PDF onto $s_{+}$and $s_{-}$are shown in Fig. 6.1(e-f).

\subsection{Systematics}

Additional systematic errors due to the analysis procedure are evaluated for the signal branching fraction, charge asymmetry, $\rho_{ \pm}$, and $\theta_{ \pm}$. The uncertainty in the model used for $\alpha\left(s_{+}, s_{-}\right)$is the largest source of error on the $C P$ parameters: $\sigma_{\rho_{ \pm}}^{\text {model }}=$ $0.03, \sigma_{\theta_{-}}^{\text {model }}=14^{\circ}, \sigma_{\theta_{+}}^{\text {model }}=11^{\circ}$. This error is evaluated by removing all but the $\rho(770), \rho(1450), f_{0}(980)$, and nonresonant terms in $\alpha\left(s_{+}, s_{-}\right)$; adding an $f_{2}^{\prime}(1525)$, an $\omega$, and a nonresonant P-wave contribution; varying the meson "radius" parameter in $F_{r}$ [43]; and propagating the errors from Table 6.1. Uncertainties due to the masses and widths of the $\rho(1700)$ and $\sigma$ resonances are small by comparison. Other errors are due to uncertainties on background yields that are fixed in the fits [42], finite MC sample size, a possible reconstruction efficiency charge asymmetry, and uncertainties in the background PDF shapes, evaluated by comparing MC and data in signal-free sidebands of the variables $M_{D}, \Delta E$, and $m_{E S}$. We also evaluate errors due to possible charge asymmetries in $D K X$ and $D K_{\mathrm{bgd}}$, uncertainties in particle identification and the efficiency functions, the finite $s_{ \pm}$measurement resolution, the background $\mathrm{PDF} f_{B}$ in the $D^{*}$ sample, $D$-flavor mistagging in the $D^{*}$ sample, and correlations between the $D$ flavor and the kaon charge in $q q_{D}$ events. These errors add in quadrature to $\sigma_{\rho_{ \pm}}^{\text {syst }}=0.05, \sigma_{\theta_{-}}^{\text {syst }}=19^{\circ}, \sigma_{\theta_{+}}^{\text {syst }}=13^{\circ}$, and are combined with the systematic errors of Eqs. (6.9).

\subsection{Validation}

The analysis procedure is validated in several ways. Conducting the analysis on the MC sample yields results consistent with the generated values. We carry out the step3 fit on a sample of $1800 \pm 70 B^{-} \rightarrow D_{\pi^{+} \pi^{-} \pi^{0}}^{0} \pi^{-}$events, obtaining the background Dalitz plot distribution from the $\Delta E$ sideband. The fit yields $\rho_{-}=0.815 \pm 0.034$, $\theta_{-}=(186 \pm 7)^{\circ}, \rho_{+}=0.854 \pm 0.035, \theta_{+}=(192 \pm 7)^{\circ}$, consistent with $\rho_{ \pm}=x_{0}$, $\theta_{ \pm}=180^{\circ}$, which corresponds to $z_{ \pm}=0$. We verify the signal efficiency by measuring the branching fraction $\mathcal{B}\left(B^{-} \rightarrow D^{0} \pi^{-}\right)$with $D^{0} \rightarrow K^{-} \pi^{+} \pi^{0}$ and $D^{0} \rightarrow \pi^{+} \pi^{-} \pi^{0}$. We compare the fit variable distributions of data and $\mathrm{MC}$ events in signal-free sidebands. Good agreement is found in all cases. 

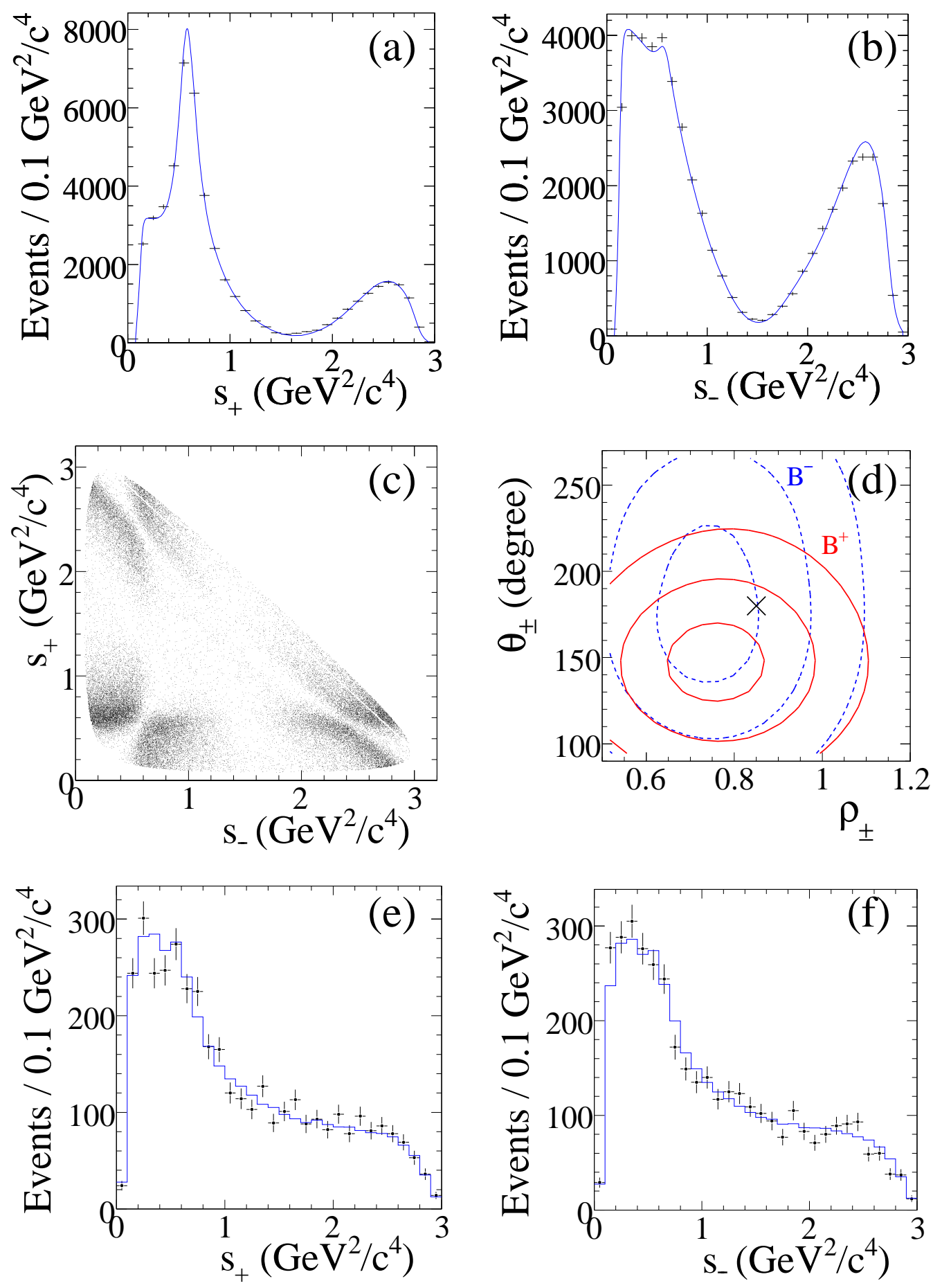

Figure 6.1: (Charge conjugation is implied for all plots.) (a,b) Projections of the $D^{*+} \rightarrow D^{0} \pi^{+}$data events and PDF onto the Dalitz plot variables $s_{+}$and $s_{-}$. (c) The 2-dimensional $\left(s_{+}, s_{-}\right)$distribution of the $D^{*+} \rightarrow D^{0} \pi^{+}$data. (d) One-, two, and three-standard-deviation contours of $\mathcal{L}$ as a function of $\theta_{ \pm}$vs. $\rho_{ \pm}$. The solid (dashed) curves correspond to $B^{+}\left(B^{-}\right)$results. The no-interference point $\left(\rho_{ \pm}=x_{0}, \theta_{ \pm}=180^{\circ}\right)$ is marked with an $\times$. (e,f) Projection of the $B^{-} \rightarrow D_{\pi^{+} \pi^{-} \pi^{0}} K^{-}$ candidate data onto $s_{+}$and $s_{-}$. 


\subsection{Results in terms of experimental parameters}

In summary, using a sample of $(324.0 \pm 3.6) \times 10^{6} e^{+} e^{-} \rightarrow B \bar{B}$ events, we observe $170 \pm 29 B^{ \pm} \rightarrow D_{\pi^{+} \pi^{-} \pi^{0}} K^{ \pm}$events. We calculate the branching fraction and decay rate asymmetry

$$
\begin{aligned}
\mathcal{B}\left(B^{ \pm} \rightarrow D_{\pi^{+} \pi^{-} \pi^{0}} K^{ \pm}\right) & =(4.6 \pm 0.8 \pm 0.4) \times 10^{-6}, \\
A\left(B^{ \pm} \rightarrow D_{\pi^{+} \pi^{-} \pi^{0}} K^{ \pm}\right) & =-0.02 \pm 0.15 \pm 0.03
\end{aligned}
$$

and the $C P$-violation parameters

$$
\begin{array}{ll}
\rho_{-}=0.72 \pm 0.11 \pm 0.06, & \theta_{-}=(173 \pm 42 \pm 19)^{\circ}, \\
\rho_{+}=0.75 \pm 0.11 \pm 0.06, & \theta_{+}=(147 \pm 23 \pm 13)^{\circ},
\end{array}
$$

where the first errors are statistical and the second are systematic. The parameters $\rho_{ \pm}, \theta_{ \pm}$are defined in Eq. (6.7). While the errors on $\theta_{ \pm}$are too large for a meaningful determination of $\gamma$ with these results alone, our errors on $\rho_{ \pm}$are small enough to make a non-negligible contribution to the overall precision of $\gamma$ in a combination of all measurements related to $\gamma$. In addition, we measure the magnitudes and phases of the components of the amplitude of the decay $D^{0} \rightarrow \pi^{+} \pi^{-} \pi^{0}$ in the isobar model.

\subsection{Constraints on physical parameters}
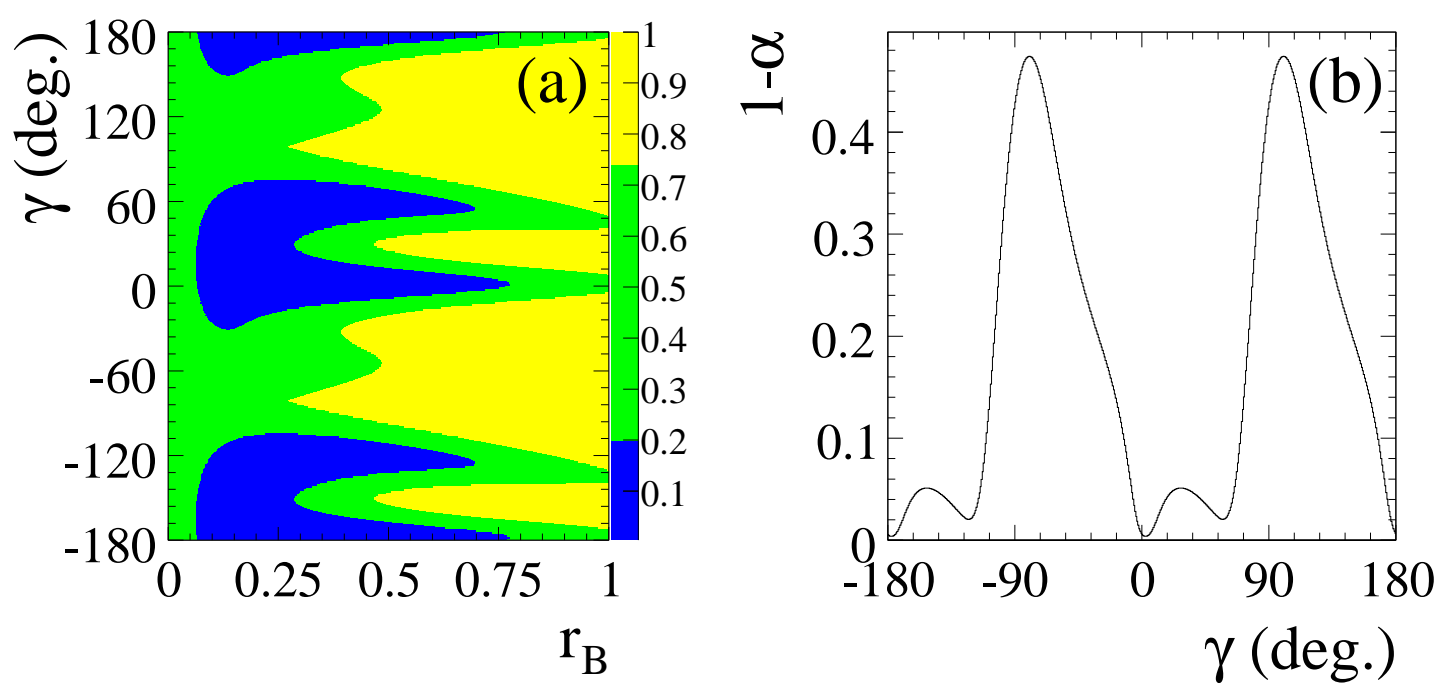

Figure 6.2: (a) Projection of the three-dimensional confidence level, $1-\alpha$, onto $r_{B}$ and $\gamma$. (b) confidence level, $1-\alpha$, versus $\gamma$.

We use the frequentist approach outlined in Ref. [39] to extract confidence regions of $\mathbf{p}=\left(r_{B}, \gamma, \delta\right)$, accounting for the dependence of the experimental errors on the values of $z_{ \pm}$and for small non-Gaussian effects in the likelihood function. Twodimensional projections onto $r_{B}$ and $\gamma$ of regions of one, two, and three standard 
deviations are shown in Fig. 6.1a. These regions are defined as containing the $\mathbf{p}$ values with three-dimensional significance $\alpha$ smaller than $19.9 \%, 73.9 \%$, and $97.1 \%$, respectively. Fig. $6.2 \mathrm{~b}$ shows the projected $\gamma$-dependence of the confidence level, $1-\alpha$. We find the one-standard-deviation regions

$$
\begin{aligned}
0.06<r_{B} & <0.78, \\
-30^{\circ}<\gamma & <76^{\circ}, \\
-27^{\circ}<\delta & <78^{\circ},
\end{aligned}
$$

including both statistical and systematic errors. Sensitivity to $r_{B}, \gamma$, and $\delta$ arises from both the Dalitz plot distribution and the signal branching fraction and asymmetry. 


\section{Chapter 7}

\section{Search for $C P$ Violation in Charm Decays}

Using $385 \mathrm{fb}^{-1}$ of $e^{+} e^{-}$collisions at the center-of-mass energies of around 10.6 $\mathrm{GeV}$, we search for time-integrated $C P$ violation in the Cabibbo-suppressed decays $D^{0} / \bar{D}^{0} \rightarrow \pi^{-} \pi^{+} \pi^{0}$ and $D^{0} / \bar{D}^{0} \rightarrow K^{-} K^{+} \pi^{0}$ using both model-independent and model-dependent methods. We find no evidence of $C P V$ at the $1 \%$ level, as expected from the Standard Model. Measured values of asymmetry in the amplitudes of flavor states and $C P$ eigenstates provide constraints on theories beyond the Standard Model some of which predict $C P$ violation in these days at the $1 \%$ level or higher.

\subsection{Motivation}

$C P$ violation $(C P V)$, manifested in an asymmetry between the decay rates of a particle and its $C P$-conjugate anti-particle, requires at least two interfering quantum mechanical amplitudes with different phases. The strong phase of each respects $C P$ symmetry while the weak phase changes sign under $C P$-conjugation. In the Standard Model (SM), the relative weak phase is typically between "tree level" and SM "penguin" amplitudes. The SM penguin amplitudes in charm decay are $\sim \mathcal{O}$ $0.1 \%$. Extensions of the SM introduce additional amplitudes which can produce $C P$ asymmetry [8] as large as $1 \%[9,10,11]$. With current experimental sensitivities $(\sim \mathcal{O} 1 \%)$, observation of $\mathrm{CPV}$ would provide a signal of new physics. The singly Cabibbo-suppressed (SCS) $D^{0}$ decays are uniquely sensitive to CPV in $c \rightarrow u \bar{d} d, u \bar{s} s$ transitions and probe contributions from supersymmetric gluonic penguins and models where up-sector quarks play a special role [9]. Such transitions can affect neither the Cabibbo favored $(c \rightarrow s \bar{d} u)$ nor the doubly Cabibbo-suppressed $(c \rightarrow d \bar{s} u)$ decays. Experimental searches $[41,45,46,47,48,49]$ are approaching this interesting level of sensitivity.

Time-integrated $C P$ asymmetries in $D^{0}$ decays can have three components: direct $\mathrm{CPV}$ in decays to specific states, indirect $\mathrm{CPV}$ in $D^{0}-\bar{D}^{0}$ mixing, and indirect CPV in interference of decays with and without mixing. Indirect CPV should be universal, but direct $\mathrm{CPV}$ can be non-universal depending on the specifics of the 
new physics [9]. We search for time-integrated CPV in the three-body, SCS decays $D^{0} / \bar{D}^{0} \rightarrow \pi^{-} \pi^{+} \pi^{0}, K^{-} K^{+} \pi^{0}$ by comparing features of $D^{0}$ amplitudes to those of $\bar{D}^{0}$. These decays proceed via both $C P$ eigenstates and flavor states, thus making it possible to probe CPV in both types of amplitudes and in the interference between them. Measuring interference effects in a Dalitz plot probes asymmetries in both the magnitudes and phases of the amplitudes, not simply in the overall decay rates. It is also insensitive to the experimental asymmetry associated with $D^{0}-\bar{D}^{0}$ mistagging. We adopt four approaches in our blind search for evidence of CPV, three of which are model-independent. First, we quantify differences between the $D^{0}$ and $\bar{D}^{0}$ Dalitz plots bin-by-bin. Second, we look for differences in the angular moments of the $D^{0}$ and $\bar{D}^{0}$ amplitudes. Third, in a model-dependent approach, we parametrize the amplitudes for $D^{0}$ and $\bar{D}^{0}$ decays separately, and look for $C P$ asymmetries in the intermediate states. Finally, we look for the phase-space-integrated asymmetry in the $D^{0}$ and $\bar{D}^{0}$ decays.

\subsection{Previous experimental studies}

Table 7.1 summarizes experimental searches for $C P$ asymmetries in various $D^{0}$ decay modes. Most of these studies looked for $C P$ asymmetry in the decay rates of two-body or quasi-two-body decays, and were therefore less sensitive to any asymmetry in the weak phase. Experimentally such a phase can show up directly in the interference effects observable in parts of the phase-space of three-body or higher multi-body decays. Since three-body decays of $D$ mesons proceed mostly via intermediate vector and scalar resonances populating different parts of the phase space (Dalitz plot [19]), it is likely that the asymmetry will be localized, and to have different magnitude and sign in different parts of the Dalitz plot. When integrated over the entire phasespace, these effects may partially, or completely, cancel each other out. Therefore, one needs to disentangle the effects of the magnitude and the phase of the new physics amplitude in the localized regions of the Dalitz plot. This requires a thorough understanding and an established parameterization of the ordinary (SM) Dalitz plot interference structure as well as knowledge of the branching fractions of the $D^{0}$ decays to these modes.

Table 7.1: Experimental measurements of direct $C P$ asymmetry in $D^{0}-\bar{D}^{0}$ decays (source: Ref. [20]).

\begin{tabular}{l|r}
\hline Decay Mode & Asymmetry \\
\hline$D^{0} / \bar{D}^{0} \rightarrow K^{+} K^{-}$ & $0.014 \pm 0.010$ \\
$D^{0} / \bar{D}^{0} \rightarrow \pi^{+} \pi^{-}$ & $0.013 \pm 0.012$ \\
$D^{0} / \bar{D}^{0} \rightarrow \pi^{0} \pi^{0}$ & $0.00 \pm 0.05$ \\
$D^{0} / \bar{D}^{0} \rightarrow \pi^{-} \pi^{+} \pi^{0}$ & $0.01 \pm 0.08$ \\
$D^{0} / \bar{D}^{0} \rightarrow K^{+} K^{-} \pi^{+} \pi^{-}$ & $-0.08 \pm 0.07$ \\
\hline
\end{tabular}




\subsection{Why three-body decays ?}

We search for direct $C P$ asymmetry in the three body, singly Cabibbo-suppressed decays to the self-conjugate final states:

$$
\begin{gathered}
D^{0} / \bar{D}^{0} \rightarrow \pi^{-} \pi^{+} \pi^{0}, \\
D^{0} / \bar{D}^{0} \rightarrow K^{-} K^{+} \pi^{0},
\end{gathered}
$$

by comparing features of $D^{0}$ decays to those of $\bar{D}^{0}$. These decays proceed via both $C P$ eigenstates (e.g., $D^{0} / \bar{D}^{0} \rightarrow \rho^{0} \pi^{0}, D^{0} / \bar{D}^{0} \rightarrow \phi(1020) \pi^{0}, D^{0} / \bar{D}^{0} \rightarrow f_{0}(980) \pi^{0}$ etc.) and non- $C P$ eigenstates (e.g., $D^{0} / \bar{D}^{0} \rightarrow \rho^{ \pm} \pi^{\mp}, D^{0} / \bar{D}^{0} \rightarrow K^{* \pm} K^{\mp}$ etc.).

We choose these channels for several reasons:

- It is possible to isolate relatively large and very pure data samples (purity $\sim 98 \%$ ).

- Small contributions to direct $C P$ asymmetry from the SM processes in the singly Cabibbo-suppressed decays make these modes particularly sensitive to new physics as described by Grossman et al. [9]: "Singly Cabibbo-suppressed $D$ decays are now more sensitive to gluonic penguin amplitudes than are the charmless $B$ decays".

- Decay modes with three-body final states are sensitive to phase information.

- The effect of charm-flavor mistagging is relatively small compared to two-body decays because the Dalitz plot is self normalized and the mistagging is symmetric between $D^{0}$ and $\bar{D}^{0}$.

- The technique of measuring interference effects in a Dalitz plot provides the tool to investigate asymmetry directly in the amplitude, not simply in the overall decay rate.

In the absence of direct $C P V$, the Dalitz plot amplitude $\mathcal{A}$ can be parameterized using the isobar model as the sum of amplitudes $A_{r}\left(s_{+}, s_{-}\right)$of all intermediate two-body resonances $r$ with complex coefficients, i.e., $\mathcal{A}=\sum_{r} a_{r} e^{i \delta_{r}} A_{r}\left(s_{+}, s_{-}\right)$. Here $s_{ \pm}$are the squared invariant masses of the pair of final state particles with charges $(+, 0)$ and $(-, 0)$, respectively. The quantities $a_{r}$ and $\delta_{r}$ are, respectively, the magnitude and phase of the complex amplitude-coefficients; they determine the relative contributions of the interfering resonances. To permit the possibility of direct $C P V$, we allow a second process - not necessarily of SM origin - to contribute to each of the amplitudes $A_{r}$, thus allowing the amplitude coefficients of $D^{0}$ and $\bar{D}^{0}$ to differ.

\subsection{Event selection}

We perform the present analysis on $385 \mathrm{fb}^{-1}$ of $e^{+} e^{-}$collision data collected at and around $10.58 \mathrm{GeV}$ center-of-mass (CM) energy with the BABAR detector [12] at the 
PEP-II storage ring. Tracking of charged particles is provided by a silicon-strip detector and a drift chamber operating in a 1.5-T magnetic field of a superconducting solenoid. Particle types are identified using specific ionization energy loss measurements in the two tracking devices and Cherenkov photons detected in a ring-imaging detector [17]. We distinguish $D^{0}$ from $\bar{D}^{0}$ by reconstructing the decays $D^{*+} \rightarrow D^{0} \pi^{+}$ and $D^{*-} \rightarrow \bar{D}^{0} \pi^{-}$.

The event-selection criteria are the same as those used in our measurement of the branching ratio of the decays $D^{0} / \bar{D}^{0} \rightarrow \pi^{-} \pi^{+} \pi^{0}, K^{-} K^{+} \pi^{0}$ [18]. In particular, we require that the $\mathrm{CM}$ momentum of the $D^{0} / \bar{D}^{0}$ candidate be greater than $2.77 \mathrm{GeV} / c$, and that $\left|m_{D^{* \pm}}-m_{D^{0} / \bar{D}^{0}}-145.4\right|<0.6 \mathrm{MeV} / c^{2}$, where $m$ refers to a reconstructed invariant mass. In the signal region, $\pm 1 \sigma$ around the reconstructed $D^{0}$ mass peak, we find $82468 \pm 321 D^{0} / \bar{D}^{0} \rightarrow \pi^{-} \pi^{+} \pi^{0}$ and $11278 \pm 110 D^{0} / \bar{D}^{0} \rightarrow K^{-} K^{+} \pi^{0}$ signal events, in both cases with a purity of about $98.1 \%$.

\subsection{Signal reconstruction efficiency}

We determine the signal reconstruction efficiency of an event as a function of the Dalitz plot variables using simulated $D^{0} / \bar{D}^{0} \rightarrow \pi^{-} \pi^{+} \pi^{0}, K^{-} K^{+} \pi^{0}$ events from $c \bar{c}$ decays, generated uniformly in the available phase space, and subjected to the same selection procedure applied to the data. In the efficiency estimation we include a binby-bin correction (in bins of momentum and polar angle of the particle tracks in the event) for any difference in the particle identification rates in data and simulation. We fit the efficiency with a cubic polynomial:

$$
\begin{aligned}
\epsilon\left(s_{+}, s_{-}\right)=1 & +s_{1}\left(s_{+}+s_{-}\right) \\
& +s_{2}\left(s_{+}^{2}+s_{-}^{2}\right)+s_{3}\left(s_{+}^{3}+s_{-}^{3}\right) \\
& +s_{4}\left(s_{-} s_{+}^{2}+s_{+} s_{-}^{2}\right)+s_{5}\left(s_{+} s_{-}\right)
\end{aligned}
$$

In addition to the relation $(7.3), \epsilon\left(s_{+}, s_{-}\right) \equiv 0$ for all points outside the physical boundary of the Dalitz plot. The result of the efficiency fit is listed in Table 7.5.

\begin{tabular}{l||r|r||r|r}
\hline \hline parameter & $D^{0} \rightarrow \pi^{-} \pi^{+} \pi^{0}$ & \multicolumn{1}{|c}{$\bar{D}^{0} \rightarrow \pi^{-} \pi^{+} \pi^{0}$} & $D^{0} \rightarrow K^{-} K^{+} \pi^{0}$ & $\bar{D}^{0} \rightarrow K^{-} K^{+} \pi^{0}$ \\
\hline$s_{1}$ & $3.47 \pm 0.38$ & $3.59 \pm 0.40$ & $-0.60 \pm 0.03$ & $-0.57 \pm 0.03$ \\
$s_{2}$ & $-1.17 \pm 0.30$ & $-1.20 \pm 0.28$ & $0.31 \pm 0.08$ & $0.28 \pm 0.09$ \\
$s_{3}$ & $0.02 \pm 0.09$ & $-0.03 \pm 0.08$ & $-0.16 \pm 0.06$ & $-0.15 \pm 0.06$ \\
$s_{4}$ & $0.43 \pm 0.21$ & $0.37 \pm 0.21$ & $0.29 \pm 0.15$ & $0.23 \pm 0.14$ \\
$s_{5}$ & $-2.08 \pm 0.54$ & $-2.27 \pm 0.51$ & $0.33 \pm 0.16$ & $0.47 \pm 0.20$ \\
\hline \hline
\end{tabular}

Table 7.2: Fit results for the efficiency coefficients of Eq. (7.3) for the signal reconstruction efficiency. 


\subsection{Sensitivity study with simulated data}

In this section, we report on the studies made using a model-independent approach using approximately $675 \mathrm{fb}^{-1}$ of $D^{0} / \bar{D}^{0} \rightarrow \pi^{-} \pi^{+} \pi^{0}$ and $440 \mathrm{fb}^{-1}$ of $D^{0} / \bar{D}^{0} \rightarrow$ $K^{-} K^{+} \pi^{0}$ simulated events. No $C P$ violation was generated in the production of the simulated signal sample, but we did introduce such effects by weighting each event by the ratio of the probability density functions with and without such $C P$ violation. We applied an "accept/reject" approach for preserving the sample to have events of weight unity.

\subsubsection{Asymmetry in two-dimensional Dalitz plot}

A straightforward comparison of the efficiency-corrected two-dimensional scatter Dalitz plots for $D^{0}$ and $\bar{D}^{0}$ events is the most intuitive way to look for $C P$ asymmetry. We compare the number of $D^{0}$ and $\bar{D}^{0}$ events observed in bins in the Dalitz plot, and plot the two-dimensional normalized residual of the difference. For this purpose, we divide the Dalitz plot in variables $m^{2}\left(h^{+} \pi^{0}, h^{-} \pi^{0}\right)$, where $h=\pi$ or $K$, into bins. We calculate normalized residual,

$$
\Delta=\left(n_{\bar{D}^{0}}-R \cdot n_{D^{0}}\right) / \sqrt{\sigma_{n_{D^{0}}}^{2}+R^{2} \cdot \sigma_{n_{D^{0}}}^{2}},
$$

in each bin, where $n$ denotes the number of events and $\sigma$ denotes statistical uncertainty. The quantity $R$ is the ratio of the total number of $\bar{D}^{0}$ to $D^{0}$ events. The factor $R$ is introduced since the total number of events in the $D^{0}$ and $\bar{D}^{0}$ Dalitz plots may differ because of production crosssection asymmetry and/or asymmetry in the branching ratio. In Figs. 7.1, we show the normalized residuals for $D^{0}$ and $\bar{D}^{0}$ Dalitz plots in simulated data. In Fig. 7.2 we show these for a $5 \%$ change in amplitude and $5^{\circ}$ difference in phase in the $D^{0}$ decays relative to the corresponding decays of $\bar{D}^{0}$ 's. This high level of $C P$ asymmetry is generated in order to clearly see the precise location(s) of asymmetry in the phase-space of the decays.
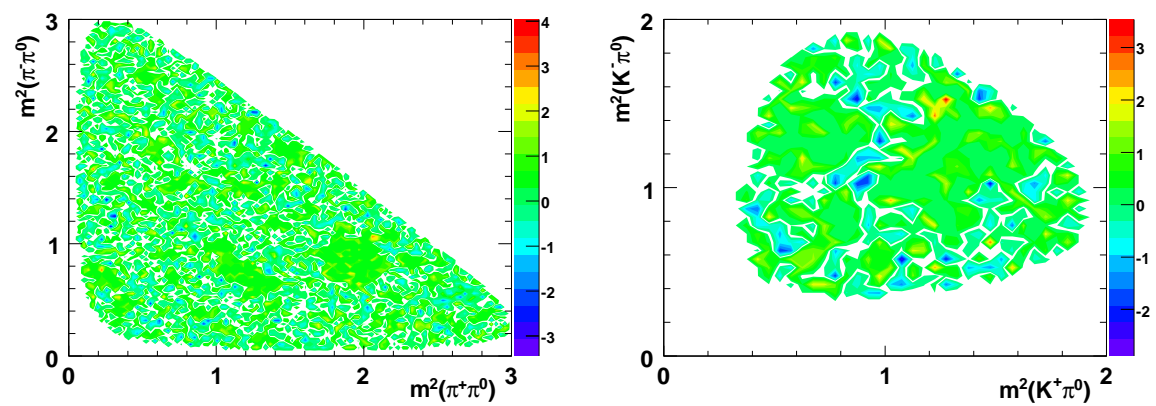

Figure 7.1: The difference between $D^{0}$ and $\bar{D}^{0}$ Dalitz plot distributions, in units of $\sigma$, for decays to the $\pi^{-} \pi^{+} \pi^{0}$ (left) and $K^{-} K^{+} \pi^{0}$ (right) final states. The amplitudes and phases for $D^{0}$ and $\bar{D}^{0}$ events are equal. The size of the simulated sample used here is roughly 25 times larger than our data sample. 

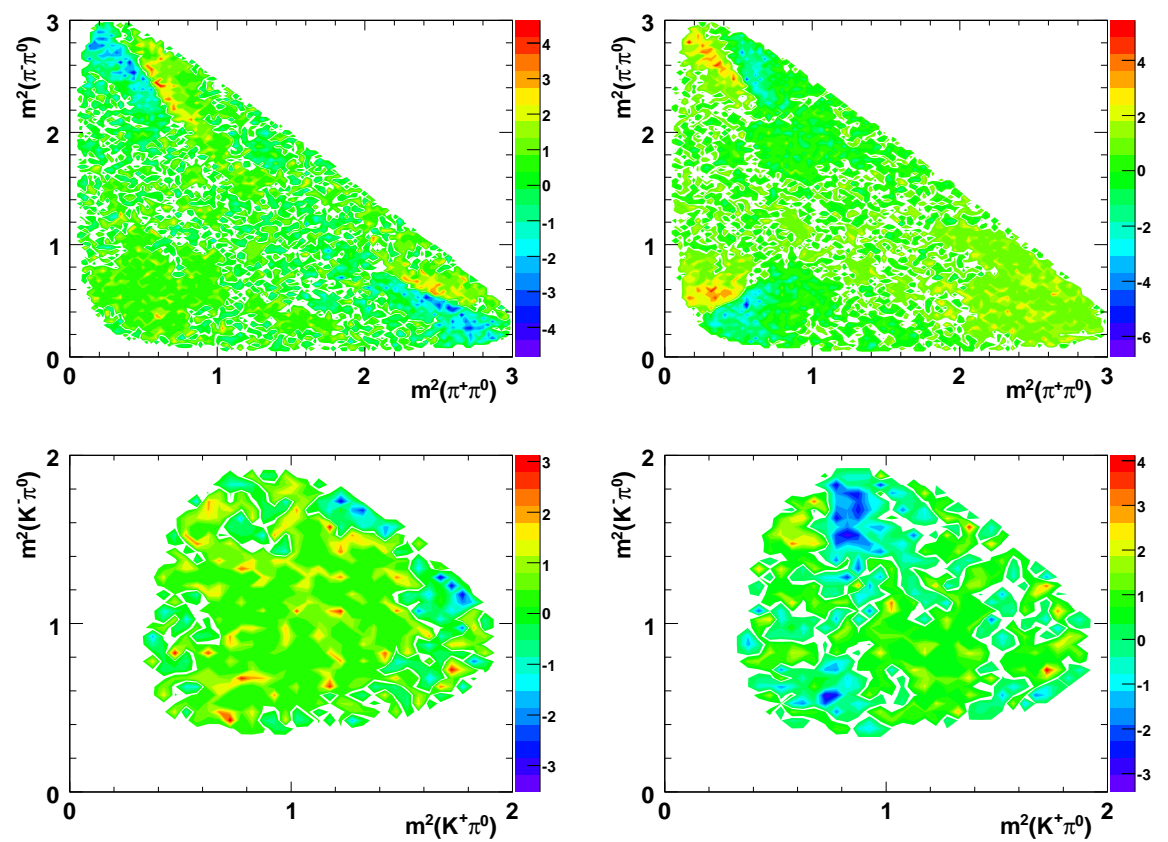

Figure 7.2: Difference between $D^{0}$ and $\bar{D}^{0}$ Dalitz plot distributions, in units of $\sigma$, for the $\pi^{-} \pi^{+} \pi^{0}$ (top) and $K^{-} K^{+} \pi^{0}$ (bottom) decays. In the top left (right) plot $\bar{D}^{0} \rightarrow$ $\rho^{0} \pi^{0}\left(\bar{D}^{0} \rightarrow \rho^{-} \pi^{+}\right)$amplitude has been changed by $+5 \%$, and its phase has been changed by $+5^{\circ}$. In the bottom left (right) plot $\bar{D}^{0} \rightarrow \phi \pi^{0}\left(\bar{D}^{0} \rightarrow K^{*}(892)^{-} K^{+}\right)$ amplitude has been changed by $+5 \%$, and its phase has been changed by $+5^{\circ}$. The size of the simulated sample used here is $\sim 25$ times larger than our data sample. 


\subsubsection{Asymmetry in distribution of angular moments}

The distribution of angular moments provides a very useful and important tool to investigate $C P$ asymmetry. It provides a model-independent framework to analyze the Dalitz plot structure without depending on the details of the parametrization. On the flip side, it is not easy to correlate the observed asymmetry in angular moments to the asymmetry in one particular intermediate resonant state.

In order to study any difference between the $D^{0}$ and $\bar{D}^{0}$ decay amplitudes, we plot the "normalized" (in number of $\sigma$ ) difference in their moments,

$$
\Delta P_{l}=\left(\overline{P_{l}}-R \cdot P_{l}\right) / \sqrt{\sigma_{P_{l}}^{2}+R^{2} \cdot \sigma_{\overline{P_{l}}}^{2}},
$$

up to order $l=7$ (i.e., the first 8 moments). The values of $\Delta P_{l}$ are expected to approach zero for larger $l$, so the value of $\chi^{2}$ obtained will depend on the chosen limit of $l$. From studies made with simulated data, we observe that effects of $C P$ violation show up predominantly in moments up to $l=7$, and that little further information is obtained above that value. We therefore limit our study to this range of moments only. We show in Fig. 7.3 the difference, $\Delta P_{l}$, for the $\pi^{-} \pi^{+} \pi^{0}$ simulated events with no $C P$ violation. As expected, the results appear to be consistent with zero. To illustrate the statistical sensitivity to $C P$ violation effects, we made similar plots for events weighted to generate $5 \%$ change in amplitude and $5^{\circ}$ difference in phase for $D^{0} \rightarrow \rho^{+} \pi^{-}\left(D^{0} \rightarrow K^{*+} K^{-}\right)$or $D^{0} \rightarrow \rho^{0} \pi^{0}\left(D^{0} \rightarrow \phi \pi^{0}\right)$ relative to the corresponding decays of $\bar{D}^{0}$ 's. These are shown in Figs. 7.4 and 7.5 for $\pi^{-} \pi^{+} \pi^{0}$ and $K^{-} K^{+} \pi^{0}$ ) decays respectively.

As observed, both model-independent methods - direct comparison of Dalitz plot distributions, and differences in moments (up to order $l=7$ ) - clearly show $C P$ violation effects if sufficiently large. In the former method, the value of $\chi^{2} / \nu$, evaluated from a binned Dalitz plot such as that in Figs. 7.1 and 7.2, serves as a figure of merit and objective test for the presence of $C P$ asymmetry.

\subsection{3 $\quad \chi^{2} / \nu$ from Dalitz plot distribution}

We compare the number of $D^{0}$ and $\bar{D}^{0}$ events observed in bins in the Dalitz plot, and plot the two-dimensional normalized residual of the difference. For this purpose, we divide the Dalitz plot in variables $m^{2}\left(h^{+} \pi^{0}, h^{-} \pi^{0}\right)$, where $h=\pi$ or $K$, into bins of size $0.05\left(\mathrm{GeV} / c^{2}\right)^{2} \times 0.05\left(\mathrm{GeV} / c^{2}\right)^{2}$. The normalized residuals are calculated as

$$
\Delta=\frac{\left(n_{\bar{D}^{0}}-R \cdot n_{D^{0}}\right)}{\sqrt{\sigma_{n_{\bar{D}^{0}}}^{2}+R^{2} \cdot \sigma_{n_{D^{0}}}^{2}}},
$$

where $n_{D^{0}}$ is the number of $D^{0}$ events, $n_{\bar{D}^{0}}$ is the number of $\bar{D}^{0}$ events in each bin and $\sigma$ is the uncertainty in the numbers. The $\chi^{2}$ of the difference in the two Dalitz plots is given by

$$
\chi^{2}=\sum_{b i n s} \Delta^{2} .
$$

Values of $\chi^{2}$ per degrees of freedom (number of non-empty bins - 1) for various cases of new physics scenarios are quoted in Table 7.3. 

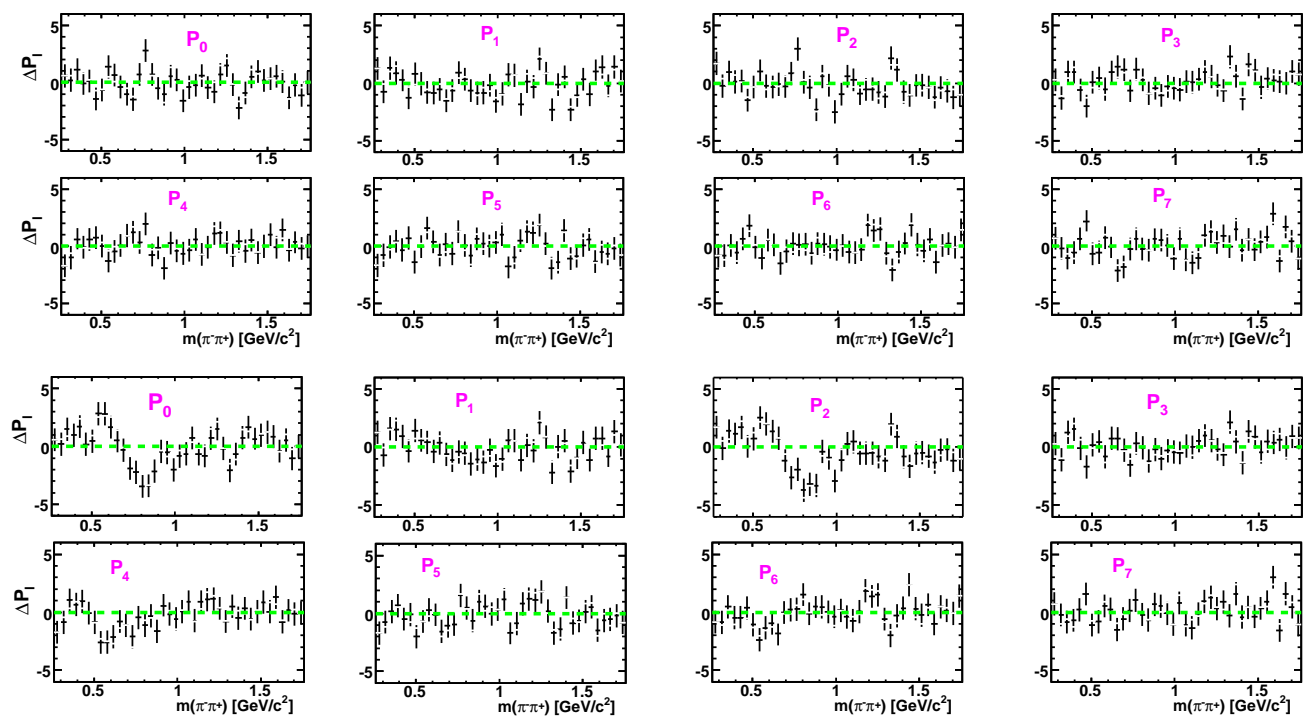

Figure 7.3: The difference in $\pi^{-} \pi^{+}$channel Legendre polynomial moments between $D^{0}$ and $\bar{D}^{0}$ decays to the $\pi^{-} \pi^{+} \pi^{0}$ final state (in number of $\sigma^{\prime}$ s). The top two rows correspond to the case of no asymmetry. In the bottom two rows, the $\bar{D}^{0} \rightarrow \rho^{0} \pi^{0}$ amplitude has been changed by $+5 \%$, and its phase has been changed by $+5^{\circ}$. The size of the simulated sample roughly corresponds to the size of our data sample.
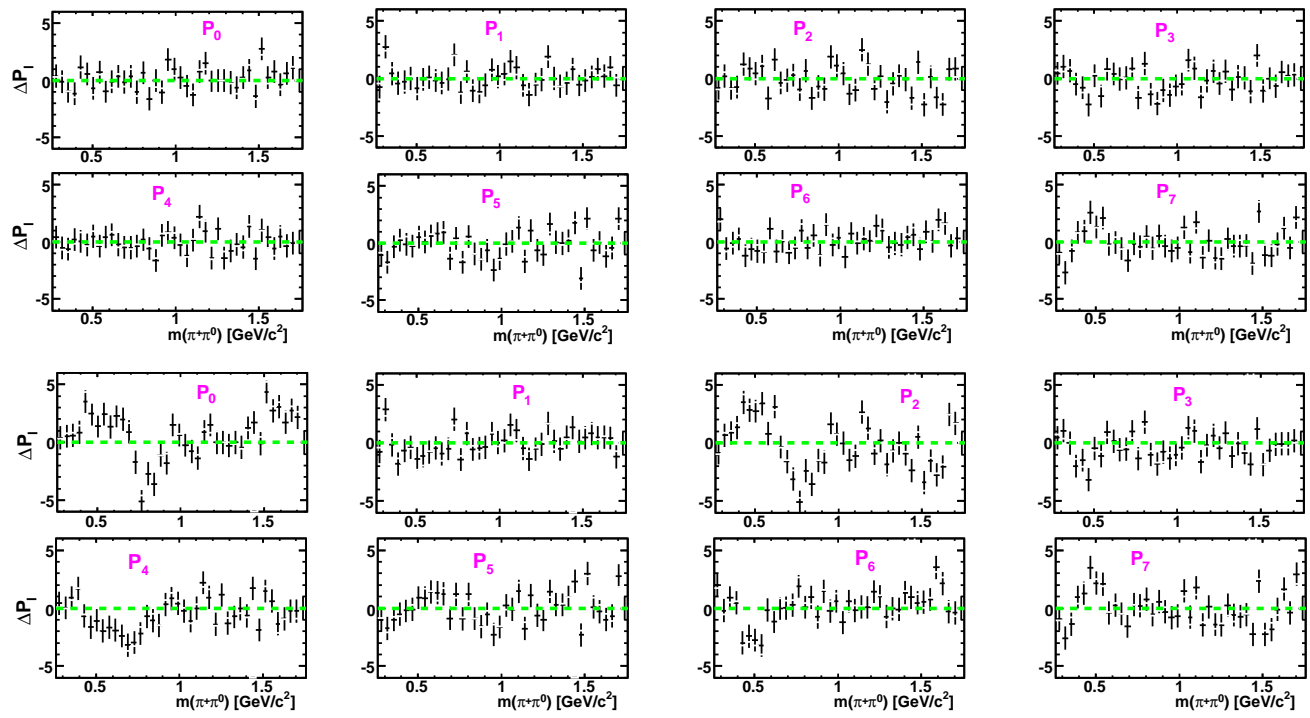

Figure 7.4: The difference in $\pi^{+} \pi^{0}$ channel Legendre polynomial moments between $D^{0}$ and $\bar{D}^{0}$ decays to the $\pi^{-} \pi^{+} \pi^{0}$ final state (in number of $\sigma^{\prime}$ s). The top two rows correspond to the case of no asymmetry. In the bottom two rows, the $\bar{D}^{0} \rightarrow \rho^{-} \pi^{+}$ amplitude has been changed by $+5 \%$, and its phase has been changed by $+5^{\circ}$. The size of the simulated sample roughly corresponds to the size of our data sample. 

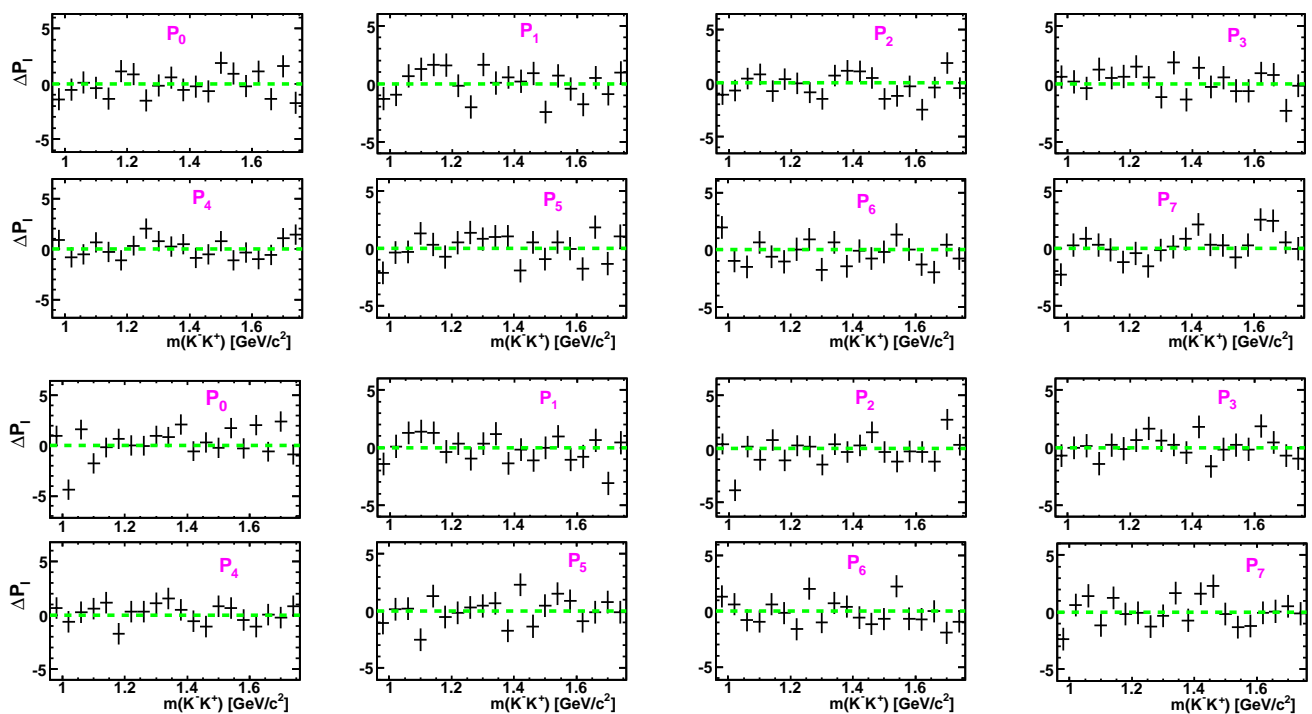

Figure 7.5: The difference in $K^{-} K^{+}$channel Legendre polynomial moments between $D^{0}$ and $\bar{D}^{0}$ decays to the $K^{-} K^{+} \pi^{0}$ final state (in number of $\sigma^{\prime}$ 's). The top two rows correspond to the case of no asymmetry. In the bottom two rows, the $\bar{D}^{0} \rightarrow \phi \pi^{0}$ amplitude has been changed by $+5 \%$, and its phase has been changed by $+5^{\circ}$. The size of the simulated sample roughly corresponds to the size of our data sample.
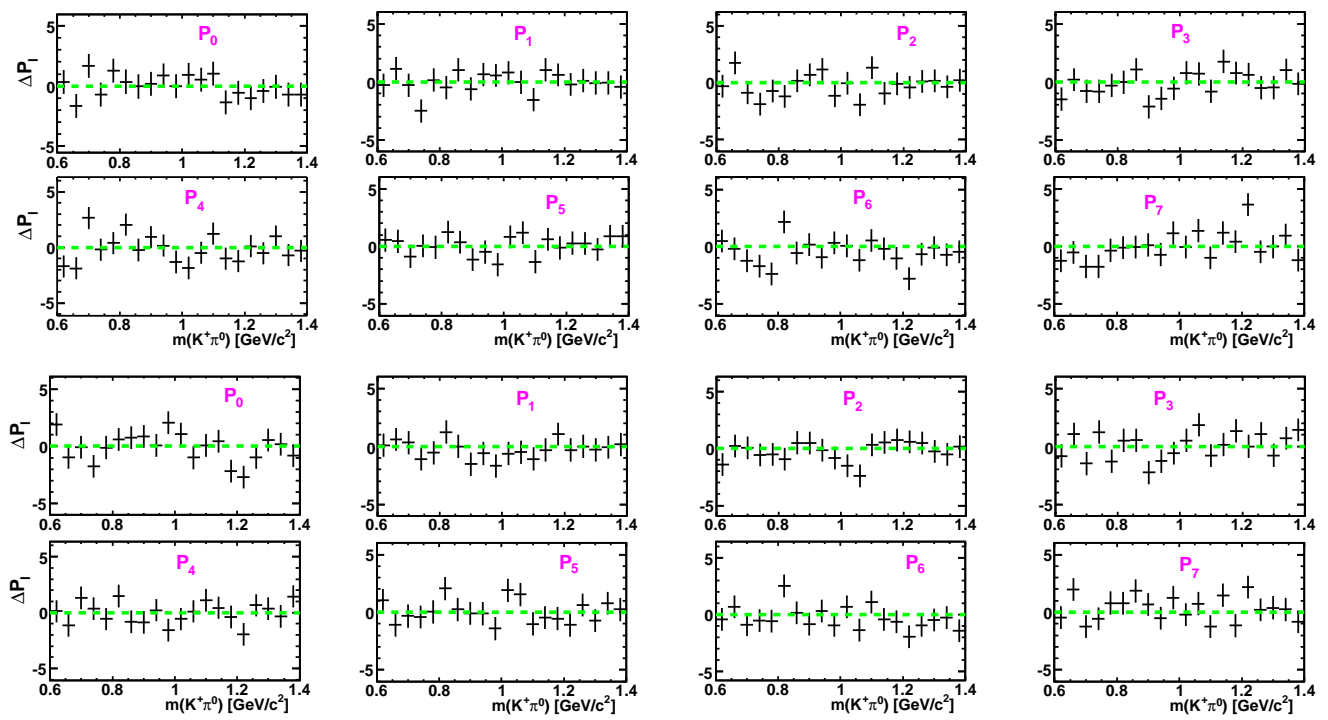

Figure 7.6: The difference in $K^{+} \pi^{0}$ channel Legendre polynomial moments between $D^{0}$ and $\bar{D}^{0}$ decays to the $K^{-} K^{+} \pi^{0}$ final state (in number of $\sigma^{\prime}$ s). The top two rows correspond to the case of no asymmetry. In the bottom two rows, the $\bar{D}^{0} \rightarrow$ $K^{*}(892)^{-} K^{+}$amplitude has been changed by $-5 \%$, and its phase has been changed by $-5^{\circ}$. The size of the simulated sample corresponds to the size of our data sample. 
Table 7.3: Model-independent $\chi^{2} / \nu$ values obtained from the difference in Dalitz plots of $D^{0}$ and $\bar{D}^{0}$ events for different levels of asymmetry in one of the intermediate states of the simulated $D^{0} \rightarrow \pi^{-} \pi^{+} \pi^{0}\left(\nu=1429\right.$, size $\left.675 \mathrm{fb}^{-1}\right)$ and $D^{0} \rightarrow K^{-} K^{+} \pi^{0}$ $\left(\nu=726\right.$, size $\left.440 \mathrm{fb}^{-1}\right)$ samples.

\begin{tabular}{|r|r|r|c|}
\hline Asymmetry in state & $\Delta a_{r} / a_{r}(\%)$ & $\Delta \phi_{r}\left(^{\circ}\right)$ & $\chi^{2} / \nu$ \\
\hline \hline$D^{0} \rightarrow \rho^{+} \pi^{-}$ & 5 & 5 & 1.57 \\
& 2 & 2 & 1.34 \\
& 1 & 1 & 1.12 \\
& 0 & 0 & 1.01 \\
\hline$D^{0} \rightarrow \rho^{0} \pi^{0}$ & 5 & 5 & 1.41 \\
& 2 & 2 & 1.19 \\
& 1 & 1 & 1.08 \\
& 0 & 0 & 1.01 \\
\hline$D^{0} \rightarrow K^{*}(892)^{+} K^{-}$ & 5 & 5 & 1.19 \\
& 2 & 2 & 1.08 \\
& 1 & 1 & 1.01 \\
& 0 & 0 & 0.99 \\
\hline$D^{0} \rightarrow \phi(1020) \pi^{0}$ & 5 & 5 & 1.27 \\
& 2 & 2 & 1.10 \\
& 1 & 1 & 1.04 \\
& 0 & 0 & 0.99 \\
\hline
\end{tabular}

\subsection{4 $\chi^{2} / \nu$ from angular moment distribution}

We then calculate a combined $\chi^{2} / \nu$ (where $\nu=320$ ) in Legendre polynomial moments for the $D^{0}$ and $\bar{D}^{0}$ samples, taking correlation among moment distributions into account.

$$
\chi^{2}=\sum_{\text {bins }} \sum_{i} \sum_{j} X_{i} \rho_{i j} X_{j}
$$

where

$$
X_{i}=\frac{\left(P_{i}^{\bar{D}^{0}}-R \cdot P_{i}^{D^{0}}\right)}{\sqrt{\sigma_{P_{i}^{D^{0}}}^{2}+R^{2} \cdot \sigma_{P_{i}^{D^{0}}}^{2}}},
$$

and $\rho_{i j}$ is the correlation coefficient between the difference in pair of moments of orders $i$ and $j$.

\subsubsection{Correlation among the angular moments}

The correlation coefficient, $\rho_{i j}$, between the difference in pair of moments of orders $i$ and $j$ is given by

$$
\rho_{i j}=\frac{\left\langle X_{i} X_{j}\right\rangle-\left\langle X_{i}\right\rangle\left\langle X_{j}\right\rangle}{\sqrt{\left\langle X_{i}^{2}\right\rangle-\left\langle X_{i}\right\rangle^{2}} \sqrt{\left\langle X_{j}^{2}\right\rangle-\left\langle X_{j}\right\rangle^{2}}}
$$


In order to determine $\left\langle X_{i}\right\rangle,\left\langle X_{i}^{2}\right\rangle$, and $\left\langle X_{i} X_{j}\right\rangle$, we generate several hundred samples (each of the size of our data sample) of simulated $D^{0}$ and $\bar{D}^{0}$ events with no asymmetry in any of the amplitudes and phases. We calculate the correlation coefficients in each of the relevant two-body invariant mass bins.

Values of $\chi^{2} / \nu$, where $\nu=320$, for various new physics scenarios are quoted in Tables 7.4-7.5. The distributions of $\chi^{2} / \nu$ for $D^{0} / \bar{D}^{0} \rightarrow \pi^{-} \pi^{+} \pi^{0}$ and $D^{0} / \bar{D}^{0} \rightarrow$ $K^{-} K^{+} \pi^{0}$ obtained from simulation are shown in Fig. 7.7 and Fig. 7.9.

Table 7.4: Model-independent $\chi^{2} / \nu$ (where $\nu=320$ ) values obtained from the difference in Legendre polynomial moments of $D^{0}$ and $\bar{D}^{0}$ events for different levels of asymmetry in one of the amplitudes $\left(\rho^{+}\right.$or $\left.\rho^{0}\right)$ of the simulated $D^{0} \rightarrow \pi^{-} \pi^{+} \pi^{0}$ sample of size $675 \mathrm{fb}^{-1}$. The amplitude ratios are in percent and the angles are in degrees.

\begin{tabular}{|c|c|c|c|c|c|c|c|}
\hline$\frac{\Delta a_{r}}{a_{r}}$ & $\Delta \phi_{r}$ & $\left(\chi^{2} / \nu\right)_{\rho^{+}}$ & $\left(\chi^{2} / \nu\right)_{\rho^{0}}$ & $\frac{\Delta a_{r}}{a_{r}}$ & $\Delta \phi_{r}$ & $\left(\chi^{2} / \nu\right)_{\rho^{+}}$ & $\left(\chi^{2} / \nu\right)_{\rho^{0}}$ \\
\hline \multirow[t]{7}{*}{-5} & 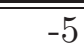 & 2.45 & $\begin{array}{l}2.12 \\
\end{array}$ & \multirow[t]{7}{*}{+5} & $\overline{-5}$ & 1.73 & $\begin{array}{ll}1.89\end{array}$ \\
\hline & -2 & 1.89 & 1.86 & & -2 & 1.59 & 1.70 \\
\hline & -1 & 1.74 & 1.84 & & -1 & 1.55 & 1.62 \\
\hline & 0 & 1.52 & 1.65 & & 0 & 1.52 & 1.51 \\
\hline & +1 & 1.70 & 1.69 & & +1 & 1.79 & 1.67 \\
\hline & +2 & 1.79 & 1.76 & & +2 & 2.09 & 1.82 \\
\hline & +5 & 2.09 & 1.96 & & +5 & 3.15 & 2.13 \\
\hline \multirow[t]{7}{*}{-2} & -5 & 2.08 & 2.11 & \multirow[t]{7}{*}{+2} & -5 & 1.80 & 1.92 \\
\hline & -2 & 1.44 & 1.60 & & -2 & 1.60 & 1.68 \\
\hline & -1 & 1.33 & 1.52 & & -1 & 1.47 & 1.47 \\
\hline & 0 & 1.14 & 1.46 & & 0 & 1.43 & 1.33 \\
\hline & +1 & 1.32 & 1.48 & & +1 & 1.62 & 1.40 \\
\hline & +2 & 1.52 & 1.61 & & +2 & 1.85 & 1.61 \\
\hline & +5 & 1.88 & 1.68 & & +5 & 2.26 & 1.80 \\
\hline \multirow[t]{7}{*}{-1} & -5 & 1.87 & 1.85 & \multirow[t]{7}{*}{+1} & -5 & 1.95 & 1.90 \\
\hline & -2 & 1.53 & 1.60 & & -2 & 1.74 & 1.73 \\
\hline & -1 & 1.49 & 1.48 & & -1 & 1.70 & 1.61 \\
\hline & 0 & 1.44 & 1.12 & & 0 & 1.38 & 1.39 \\
\hline & +1 & 1.56 & 1.51 & & +1 & 1.54 & 1.47 \\
\hline & +2 & 1.74 & 1.79 & & +2 & 1.64 & 1.60 \\
\hline & +5 & 2.03 & 1.90 & & +5 & 1.80 & 2.10 \\
\hline \multirow[t]{4}{*}{0} & -5 & 2.05 & 1.81 & \multirow[t]{4}{*}{0} & +5 & 2.04 & 1.84 \\
\hline & -2 & 1.51 & 1.55 & & +2 & 1.44 & 1.58 \\
\hline & -1 & 1.28 & 1.29 & & +1 & 1.24 & 1.25 \\
\hline & 0 & 1.03 & 1.03 & & & & \\
\hline
\end{tabular}

\subsubsection{Model dependent analysis on simulated data}

We the simulated data sample using exactly the same procedure and phase conventions as in the previous chapters. The results of the fit, for various cases of 
Table 7.5: Model-independent $\chi^{2} / \nu$ (where $\nu=160$ ) values obtained from the difference in Legendre polynomial moments of $D^{0}$ and $\bar{D}^{0}$ events for different levels of asymmetry in one of the amplitudes $\left(K^{*}(892)^{+}\right.$or $\left.\phi(1020)\right)$ of the simulated $D^{0} \rightarrow K^{-} K^{+} \pi^{0}$ sample of size $440 \mathrm{fb}^{-1}$. The amplitude ratios are in percent and the angles are in degrees.

\begin{tabular}{|rrrr|rrrr|}
\hline$\frac{\Delta a_{r}}{a_{r}}$ & $\Delta \phi_{r}$ & $\left(\chi^{2} / \nu\right)_{K^{*}}$ & $\left(\chi^{2} / \nu\right)_{\phi}$ & $\frac{\Delta a_{r}}{a_{r}}$ & $\Delta \phi_{r}$ & $\left(\chi^{2} / \nu\right)_{K^{*}}$ & $\left(\chi^{2} / \nu\right)_{\phi}$ \\
\hline \hline-5 & -5 & 1.94 & 1.86 & +5 & -5 & 1.86 & 1.82 \\
& -2 & 1.80 & 1.72 & & -2 & 1.80 & 1.76 \\
& -1 & 1.74 & 1.65 & & -1 & 1.72 & 1.64 \\
& 0 & 1.67 & 1.54 & & 0 & 1.49 & 1.46 \\
+1 & 1.78 & 1.58 & & +1 & 1.64 & 1.64 \\
& +2 & 1.82 & 1.63 & & +2 & 1.72 & 1.88 \\
& +5 & 2.07 & 1.85 & & +5 & 1.87 & 1.99 \\
\hline-2 & -5 & 1.81 & 1.81 & +2 & -5 & 1.88 & 1.72 \\
& -2 & 1.72 & 1.70 & & -2 & 1.74 & 1.59 \\
& -1 & 1.63 & 1.64 & & -1 & 1.66 & 1.43 \\
& 0 & 1.49 & 1.49 & & 0 & 1.32 & 1.31 \\
& +1 & 1.56 & 1.61 & & +1 & 1.46 & 1.42 \\
& +2 & 1.70 & 1.75 & & +2 & 1.70 & 1.44 \\
+5 & 1.74 & 1.82 & & +5 & 1.82 & 1.53 \\
\hline-1 & -5 & 1.74 & 1.60 & +1 & -5 & 1.79 & 1.64 \\
& -2 & 1.69 & 1.52 & & -2 & 1.67 & 1.51 \\
& -1 & 1.43 & 1.47 & & -1 & 1.54 & 1.21 \\
& 0 & 1.24 & 1.35 & & 0 & 1.22 & 1.17 \\
& 1 & 1.29 & 1.49 & & +1 & 1.41 & 1.24 \\
& +2 & 1.42 & 1.56 & & +2 & 1.63 & 1.30 \\
& 1.62 & 1.63 & & +5 & 1.72 & 1.58 \\
\hline 0 & -5 & 1.54 & 1.48 & 0 & +5 & 1.40 & 1.42 \\
& -2 & 1.51 & 1.31 & & +2 & 1.24 & 1.28 \\
& 1 & 1.34 & 1.17 & & +1 & 1.14 & 1.13 \\
& 0 & 1.02 & 1.02 & & & & \\
\hline
\end{tabular}



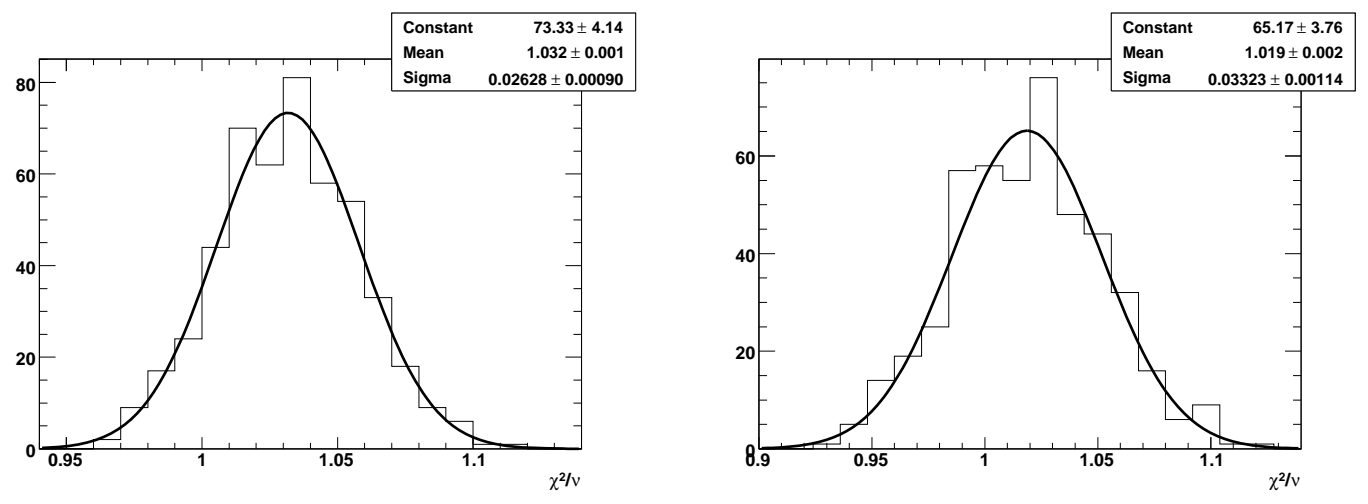

Figure 7.7: $\chi^{2} / \nu$ for difference in angular moments in case of no $C P$ asymmetry obtained from simulation: $\pi^{+} \pi^{-}$channel of $D \rightarrow \pi^{-} \pi^{+} \pi^{0}$ (left), $K^{+} K^{-}$channel of $D \rightarrow K^{-} K^{+} \pi^{0}$ (right).
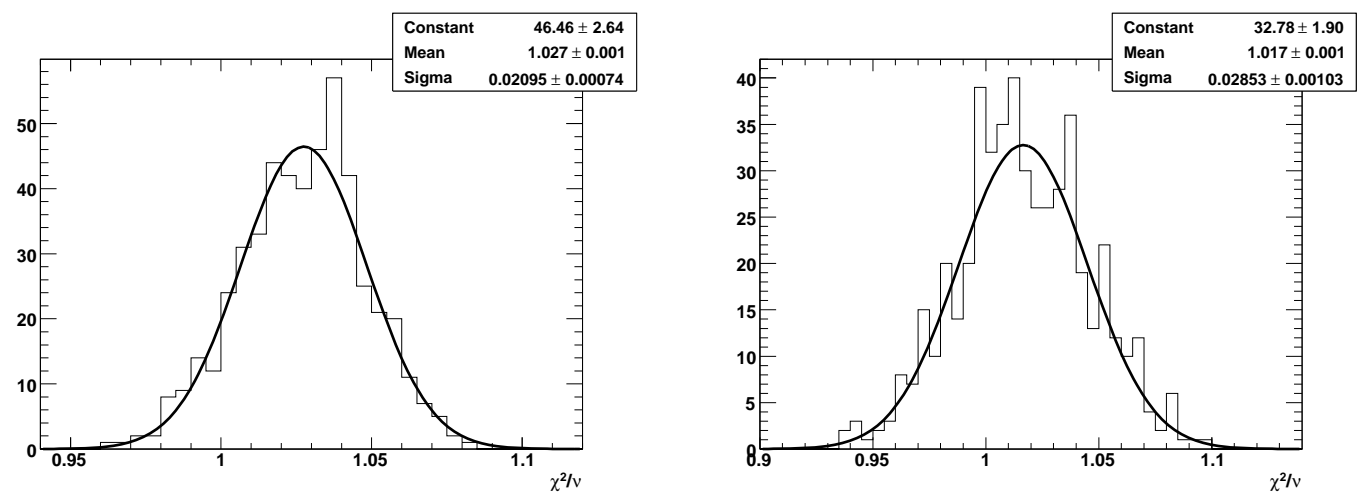

Figure 7.8: $\chi^{2} / \nu$ for difference in angular moments in case of no $C P$ asymmetry obtained from simulation: $\pi^{+} \pi^{0}$ channel of $D \rightarrow \pi^{-} \pi^{+} \pi^{0}$ (left), $K^{+} \pi^{0}$ channel of $D \rightarrow K^{-} K^{+} \pi^{0}$ (right).
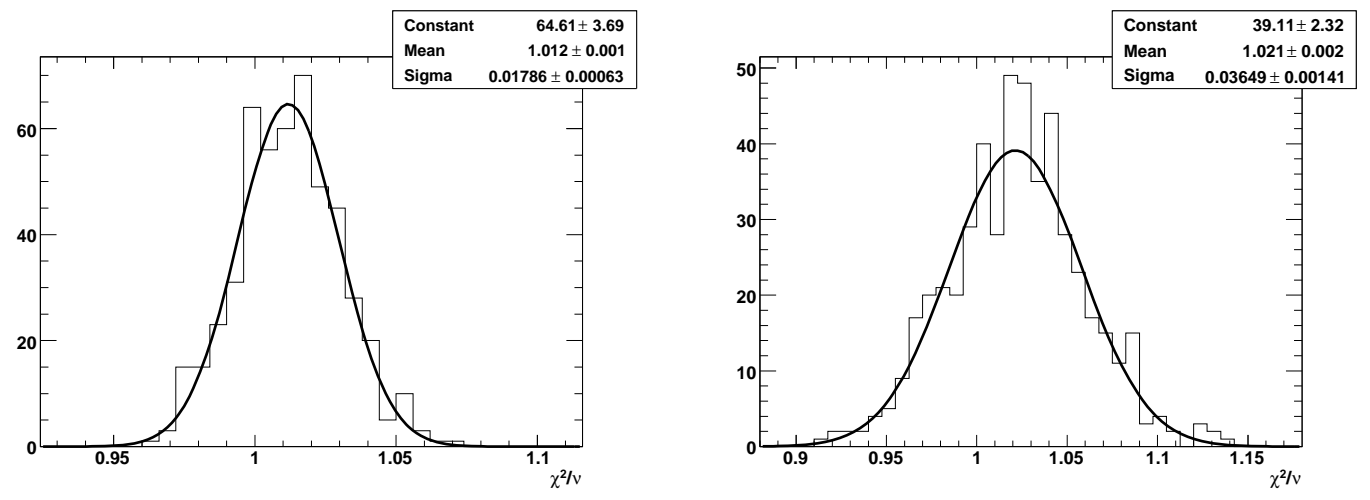

Figure 7.9: $\chi^{2} / \nu$ for difference in Dalitz plot structure in case of no $C P$ asymmetry obtained from simulation: $D \rightarrow \pi^{-} \pi^{+} \pi^{0}$ (left), $D \rightarrow K^{-} K^{+} \pi^{0}$ (right). 
asymmetry are listed in the Tables 7.6-7.9. It is clear from these results that we are able to get generated parameters back from the fit for all studied cases of $C P$ violation effects. We also observe that we have statistically significant sensitivity in determining the phase-shift and amplitude variation in the dominant decay modes of the $D^{0} / \bar{D}^{0} \rightarrow \pi^{-} \pi^{+} \pi^{0}$ decay if such changes are more than $1 \%$ in amplitude or more than $1^{\circ}$ in phase. As expected, the sensitivity for detecting either phase-shift or amplitude variation in the $D^{0} / \bar{D}^{0} \rightarrow K^{-} K^{+} \pi^{0}$ decay is substantially smaller than that in $D^{0} \rightarrow \pi^{-} \pi^{+} \pi^{0}$. However, if the asymmetry is large $\left(\sim 5 \%\right.$ or $\left.5^{\circ}\right)$ in any dominant decay mode, then we should be able to observe it with good sensitivity even in the $D^{0} / \bar{D}^{0} \rightarrow K^{-} K^{+} \pi^{0}$ decay.

Table 7.6: Model-dependent asymmetry values obtained from the likelihood fit for different levels of generated asymmetry in the $D^{0} \rightarrow \rho^{0} \pi^{0}$ state in the Monte Carlo $D^{0} \rightarrow \pi^{-} \pi^{+} \pi^{0}$ sample of size $675 \mathrm{fb}^{-1}$. The amplitude ratios are in percent and the angles are in degrees.

\begin{tabular}{|c|c|c|c|c|c|c|c|}
\hline & Gen & \multicolumn{2}{|l|}{ Fit } & \multicolumn{4}{|c|}{ Gen } \\
\hline$\frac{\Delta a_{r}}{a_{r}}$ & $\Delta \phi_{r}$ & $\frac{\Delta a_{r}}{a_{r}}$ & $\Delta \phi_{r}$ & $\frac{\Delta a_{r}}{a_{r}}$ & $\Delta \phi_{r}$ & $\frac{\Delta a_{r}}{a_{r}}$ & $\Delta \phi_{r}$ \\
\hline \multirow[t]{7}{*}{-5} & -5 & $\overline{-5.97 \pm 0.56}$ & $\overline{-4.74 \pm 0.39}$ & \multirow[t]{7}{*}{+5} & -5 & $+5.53 \pm 0.62$ & $\overline{-5.36 \pm 0.47}$ \\
\hline & -2 & $-4.84 \pm 0.58$ & $-1.92 \pm 0.38$ & & -2 & $+4.71 \pm 0.65$ & $-2.65 \pm 0.41$ \\
\hline & -1 & $-5.60 \pm 0.58$ & $-1.36 \pm 0.42$ & & -1 & $+5.20 \pm 0.56$ & $-0.86 \pm 0.40$ \\
\hline & 0 & $-5.75 \pm 0.60$ & $+0.22 \pm 0.41$ & & 0 & $+4.40 \pm 0.58$ & $-0.23 \pm 0.40$ \\
\hline & +1 & $-4.65 \pm 0.60$ & $+0.79 \pm 0.43$ & & +1 & $+5.08 \pm 0.66$ & $+1.22 \pm 0.48$ \\
\hline & +2 & $-6.08 \pm 0.58$ & $+2.44 \pm 0.45$ & & +2 & $+4.57 \pm 0.62$ & $+1.89 \pm 0.42$ \\
\hline & +5 & $-4.83 \pm 0.61$ & $+4.32 \pm 0.42$ & & +5 & $+4.15 \pm 0.63$ & $+4.52 \pm 0.44$ \\
\hline \multirow[t]{7}{*}{-2} & -5 & $-1.65 \pm 0.58$ & $-5.86 \pm 0.46$ & \multirow[t]{7}{*}{+2} & -5 & $+1.45 \pm 0.58$ & $-4.45 \pm 0.45$ \\
\hline & -2 & $-2.25 \pm 0.60$ & $-2.69 \pm 0.41$ & & -2 & $+1.66 \pm 0.61$ & $-2.81 \pm 0.44$ \\
\hline & -1 & $-3.23 \pm 0.61$ & $-1.03 \pm 0.44$ & & -1 & $+2.47 \pm 0.61$ & $-1.51 \pm 0.42$ \\
\hline & 0 & $-2.08 \pm 0.60$ & $-0.65 \pm 0.42$ & & 0 & $+2.04 \pm 0.58$ & $-0.38 \pm 0.45$ \\
\hline & +1 & $-2.59 \pm 0.63$ & $+1.38 \pm 0.43$ & & +1 & $+1.97 \pm 0.61$ & $+0.41 \pm 0.46$ \\
\hline & +2 & $-1.67 \pm 0.61$ & $+1.64 \pm 0.43$ & & +2 & $+1.51 \pm 0.60$ & $+1.57 \pm 0.42$ \\
\hline & +5 & $-3.25 \pm 0.63$ & $+4.69 \pm 0.42$ & & +5 & $+2.51 \pm 0.64$ & $+5.91 \pm 0.48$ \\
\hline \multirow[t]{7}{*}{-1} & -5 & $-1.62 \pm 0.54$ & $-4.26 \pm 0.44$ & \multirow[t]{7}{*}{+1} & -5 & $+0.14 \pm 0.60$ & $-4.80 \pm 0.42$ \\
\hline & -2 & $-1.26 \pm 0.60$ & $-2.18 \pm 0.46$ & & -2 & $+1.09 \pm 0.61$ & $-1.74 \pm 0.43$ \\
\hline & -1 & $-0.87 \pm 0.63$ & $+0.04 \pm 0.39$ & & -1 & $+1.31 \pm 0.62$ & $-0.44 \pm 0.45$ \\
\hline & 0 & $-1.05 \pm 0.54$ & $+0.31 \pm 0.40$ & & 0 & $+0.92 \pm 0.63$ & $+0.64 \pm 0.44$ \\
\hline & +1 & $-1.58 \pm 0.54$ & $+0.63 \pm 0.47$ & & +1 & $+0.34 \pm 0.61$ & $+1.83 \pm 0.44$ \\
\hline & +2 & $-0.72 \pm 0.58$ & $+1.65 \pm 0.42$ & & +2 & $+1.27 \pm 0.61$ & $+2.64 \pm 0.46$ \\
\hline & +5 & $-1.10 \pm 0.58$ & $+4.62 \pm 0.43$ & & +5 & $+0.60 \pm 0.65$ & $+5.55 \pm 0.42$ \\
\hline \multirow[t]{4}{*}{0} & -5 & $-0.22 \pm 0.56$ & $-6.11 \pm 0.45$ & \multirow[t]{4}{*}{0} & +5 & $-0.49 \pm 0.63$ & $+4.08 \pm 0.44$ \\
\hline & -2 & $-0.81 \pm 0.61$ & $-2.85 \pm 0.47$ & & +2 & $+0.89 \pm 0.62$ & $+1.69 \pm 0.43$ \\
\hline & -1 & $+0.57 \pm 0.63$ & $-0.82 \pm 0.48$ & & +1 & $+0.34 \pm 0.58$ & $+1.15 \pm 0.43$ \\
\hline & 0 & $+0.14 \pm 0.60$ & $+0.29 \pm 0.45$ & & & & \\
\hline
\end{tabular}


Table 7.7: Model-dependent asymmetry values obtained from the likelihood fit for different levels of generated asymmetry in the $D^{0} \rightarrow \rho^{+} \pi^{-}$state in the Monte Carlo $D^{0} \rightarrow \pi^{-} \pi^{+} \pi^{0}$ sample of size $675 \mathrm{fb}^{-1}$. The amplitude ratios are in percent and the angles are in degrees.

\begin{tabular}{|c|c|c|c|c|c|c|c|}
\hline & Gen & \multicolumn{2}{|l|}{ Fit } & \multicolumn{2}{|r|}{ Gen } & \multicolumn{2}{|l|}{ Fit } \\
\hline$\frac{\Delta a_{r}}{a_{r}}$ & $\Delta \phi_{r}$ & $\frac{\Delta a_{r}}{a_{r}}$ & $\Delta \phi_{r}$ & $\frac{\Delta a_{r}}{a_{r}}$ & $\Delta \phi_{r}$ & $\frac{\Delta a_{r}}{a_{r}}$ & $\Delta \phi_{r}$ \\
\hline \multirow[t]{7}{*}{-5} & -5 & $-4.27 \pm 0.67$ & $-4.91 \pm 0.28$ & \multirow[t]{7}{*}{+5} & -5 & $+5.30 \pm 0.67$ & $-4.61 \pm 0.34$ \\
\hline & -2 & $-4.45 \pm 0.68$ & $-1.40 \pm 0.29$ & & -2 & $+5.27 \pm 0.69$ & $-1.74 \pm 0.35$ \\
\hline & -1 & $-5.10 \pm 0.61$ & $-0.66 \pm 0.29$ & & -1 & $+4.48 \pm 0.66$ & $-0.44 \pm 0.38$ \\
\hline & 0 & $-4.61 \pm 0.68$ & $-0.10 \pm 0.31$ & & 0 & $+4.31 \pm 0.68$ & $+0.33 \pm 0.37$ \\
\hline & +1 & $-5.88 \pm 0.64$ & $+1.49 \pm 0.33$ & & +1 & $+4.37 \pm 0.69$ & $+1.28 \pm 0.39$ \\
\hline & +2 & $-5.07 \pm 0.59$ & $+2.60 \pm 0.29$ & & +2 & $+5.64 \pm 0.61$ & $+2.64 \pm 0.38$ \\
\hline & +5 & $-4.52 \pm 0.65$ & $+5.69 \pm 0.29$ & & +5 & $+5.02 \pm 0.64$ & $+5.58 \pm 0.32$ \\
\hline \multirow[t]{7}{*}{-2} & -5 & $-2.10 \pm 0.68$ & $-4.96 \pm 0.32$ & \multirow[t]{7}{*}{+2} & -5 & $+1.06 \pm 0.63$ & $-4.81 \pm 0.32$ \\
\hline & -2 & $-1.12 \pm 0.70$ & $-1.48 \pm 0.34$ & & -2 & $+2.07 \pm 0.66$ & $-1.57 \pm 0.34$ \\
\hline & -1 & $-2.00 \pm 0.61$ & $-0.24 \pm 0.35$ & & -1 & $+1.68 \pm 0.65$ & $-0.75 \pm 0.34$ \\
\hline & 0 & $-1.83 \pm 0.60$ & $-0.44 \pm 0.29$ & & 0 & $+2.18 \pm 0.65$ & $-0.54 \pm 0.33$ \\
\hline & +1 & $-1.33 \pm 0.61$ & $+1.21 \pm 0.31$ & & +1 & $+2.52 \pm 0.67$ & $+1.42 \pm 0.34$ \\
\hline & +2 & $-1.94 \pm 0.65$ & $+2.40 \pm 0.32$ & & +2 & $+2.19 \pm 0.67$ & $+2.41 \pm 0.34$ \\
\hline & +5 & $-2.45 \pm 0.66$ & $+5.17 \pm 0.32$ & & +5 & $+2.10 \pm 0.60$ & $+5.42 \pm 0.37$ \\
\hline \multirow[t]{7}{*}{-1} & -5 & $-0.80 \pm 0.69$ & $-4.40 \pm 0.38$ & \multirow[t]{7}{*}{+1} & -5 & $+1.28 \pm 0.67$ & $-4.49 \pm 0.39$ \\
\hline & -2 & $-0.45 \pm 0.62$ & $-2.01 \pm 0.31$ & & -2 & $+1.50 \pm 0.60$ & $-1.86 \pm 0.31$ \\
\hline & -1 & $-1.26 \pm 0.63$ & $-0.62 \pm 0.30$ & & -1 & $+0.90 \pm 0.69$ & $-0.80 \pm 0.30$ \\
\hline & 0 & $-0.93 \pm 0.65$ & $+0.10 \pm 0.31$ & & 0 & $+1.72 \pm 0.63$ & $+0.32 \pm 0.33$ \\
\hline & +1 & $-1.08 \pm 0.67$ & $+1.19 \pm 0.39$ & & +1 & $+1.74 \pm 0.63$ & $+1.24 \pm 0.34$ \\
\hline & +2 & $-0.98 \pm 0.64$ & $+2.18 \pm 0.31$ & & +2 & $+1.66 \pm 0.64$ & $+1.95 \pm 0.33$ \\
\hline & +5 & $-1.17 \pm 0.69$ & $+5.53 \pm 0.34$ & & +5 & $+1.10 \pm 0.67$ & $+5.72 \pm 0.35$ \\
\hline \multirow[t]{4}{*}{0} & -5 & $-0.96 \pm 0.65$ & $-5.40 \pm 0.30$ & \multirow[t]{4}{*}{0} & +5 & $+0.44 \pm 0.63$ & $+5.62 \pm 0.36$ \\
\hline & -2 & $+0.29 \pm 0.64$ & $-1.80 \pm 0.32$ & & +2 & $+0.81 \pm 0.62$ & $+2.23 \pm 0.34$ \\
\hline & -1 & $+0.45 \pm 0.67$ & $-1.13 \pm 0.30$ & & +1 & $-0.26 \pm 0.69$ & $+1.63 \pm 0.30$ \\
\hline & 0 & $+0.74 \pm 0.68$ & $-0.16 \pm 0.32$ & & & & \\
\hline
\end{tabular}


Table 7.8: Model-dependent asymmetry values obtained from the likelihood fit for different levels of generated asymmetry in the $D^{0} \rightarrow \phi(1020) \pi^{0}$ state in the Monte Carlo $D^{0} \rightarrow K^{-} K^{+} \pi^{0}$ sample of size $440 \mathrm{fb}^{-1}$. The amplitude ratios are in percent and the angles are in degrees.

\begin{tabular}{|rr|rr||rr|rr|}
\hline & Gen & \multicolumn{2}{|c|}{ Fit } & & Gen & \multicolumn{2}{c|}{ Fit } \\
\hline$\frac{\Delta a_{r}}{a_{r}}$ & $\Delta \phi_{r}$ & $\frac{\Delta a_{r}}{a_{r}}$ & $\Delta \phi_{r}$ & $\frac{\Delta a_{r}}{a_{r}}$ & $\Delta \phi_{r}$ & $\frac{\Delta a_{r}}{a_{r}}$ & $\Delta \phi_{r}$ \\
\hline \hline-5 & -5 & $-3.79 \pm 1.05$ & $-5.70 \pm 1.82$ & +5 & -5 & $+6.84 \pm 1.13$ & $-5.94 \pm 1.68$ \\
& -2 & $-5.05 \pm 1.06$ & $-1.45 \pm 1.75$ & -2 & $+5.48 \pm 1.12$ & $-3.72 \pm 1.70$ \\
& -1 & $-6.85 \pm 1.05$ & $-1.62 \pm 1.71$ & -1 & $+4.19 \pm 1.09$ & $-2.30 \pm 1.71$ \\
& 0 & $-4.18 \pm 1.08$ & $-0.79 \pm 1.74$ & & 0 & $+5.75 \pm 1.10$ & $+0.68 \pm 1.68$ \\
& +1 & $-7.10 \pm 1.11$ & $+1.94 \pm 1.77$ & +1 & $+3.88 \pm 1.14$ & $+1.59 \pm 1.67$ \\
& +2 & $-5.32 \pm 1.06$ & $+3.61 \pm 1.76$ & & +2 & $+4.44 \pm 1.05$ & $+3.07 \pm 1.69$ \\
& +5 & $-3.85 \pm 1.07$ & $+5.88 \pm 1.78$ & & +5 & $+4.89 \pm 1.08$ & $+4.44 \pm 1.70$ \\
\hline-2 & -5 & $-2.86 \pm 1.06$ & $-6.87 \pm 1.74$ & +2 & -5 & $+2.14 \pm 1.04$ & $-4.52 \pm 1.76$ \\
& -2 & $-3.32 \pm 1.05$ & $-3.48 \pm 1.73$ & & -2 & $+1.88 \pm 1.05$ & $-3.36 \pm 1.65$ \\
& -1 & $-1.39 \pm 1.05$ & $+0.62 \pm 1.74$ & -1 & $+1.27 \pm 1.12$ & $-0.86 \pm 1.73$ \\
& 0 & $-1.78 \pm 1.04$ & $-0.21 \pm 1.68$ & & 0 & $+2.96 \pm 1.07$ & $-0.11 \pm 1.75$ \\
& +1 & $-2.14 \pm 1.07$ & $+0.24 \pm 1.70$ & +1 & $+2.38 \pm 1.10$ & $+0.09 \pm 1.72$ \\
& +2 & $-1.65 \pm 1.10$ & $+1.11 \pm 1.73$ & +2 & $+1.44 \pm 1.09$ & $+2.88 \pm 1.70$ \\
+5 & $-2.22 \pm 1.08$ & $+3.31 \pm 1.74$ & & +5 & $+1.78 \pm 1.06$ & $+6.73 \pm 1.71$ \\
\hline-1 & -5 & $+0.20 \pm 1.11$ & $-4.08 \pm 1.72$ & +1 & -5 & $+0.43 \pm 1.04$ & $-5.75 \pm 1.75$ \\
& -2 & $-1.64 \pm 1.09$ & $-1.31 \pm 1.76$ & -2 & $+0.87 \pm 1.07$ & $-1.96 \pm 1.71$ \\
& -1 & $-0.76 \pm 1.09$ & $-1.22 \pm 1.73$ & -1 & $+1.59 \pm 1.05$ & $-1.49 \pm 1.67$ \\
0 & $-0.49 \pm 1.10$ & $-0.01 \pm 1.72$ & & 0 & $+1.88 \pm 1.06$ & $-0.37 \pm 1.71$ \\
& +1 & $-2.01 \pm 1.08$ & $+1.85 \pm 1.74$ & +1 & $+0.29 \pm 1.04$ & $+0.17 \pm 1.71$ \\
+2 & $-1.24 \pm 1.07$ & $+2.16 \pm 1.70$ & +2 & $+0.95 \pm 1.05$ & $+1.24 \pm 1.71$ \\
+5 & $-0.91 \pm 1.08$ & $+4.49 \pm 1.73$ & +5 & $+1.95 \pm 1.09$ & $+4.11 \pm 1.68$ \\
\hline 0 & -5 & $+0.32 \pm 1.06$ & $-4.37 \pm 1.74$ & 0 & +5 & $+0.18 \pm 1.05$ & $+3.99 \pm 1.74$ \\
-2 & $-0.81 \pm 1.04$ & $-3.16 \pm 1.75$ & +2 & $-0.02 \pm 1.06$ & $+2.06 \pm 1.72$ \\
-1 & $-0.25 \pm 1.05$ & $-2.03 \pm 1.71$ & +1 & $+0.76 \pm 1.07$ & $+0.15 \pm 1.71$ \\
0 & $+0.54 \pm 1.07$ & $-0.82 \pm 1.69$ & & & \\
\hline
\end{tabular}


Table 7.9: Model-dependent asymmetry values obtained from the likelihood fit for different levels of generated asymmetry in the $D^{0} \rightarrow K^{*}(892)^{+} K^{-}$state in the Monte Carlo $D^{0} \rightarrow K^{-} K^{+} \pi^{0}$ sample of size $440 \mathrm{fb}^{-1}$. The amplitude ratios are in percent and the angles are in degrees.

\begin{tabular}{|rr|rr||rr|rr|}
\hline & Gen & \multicolumn{2}{|c||}{ Fit } & & Gen & \multicolumn{2}{|c|}{ Fit } \\
\hline$\frac{\Delta a_{r}}{a_{r}}$ & $\Delta \phi_{r}$ & $\frac{\Delta a_{r}}{a_{r}}$ & $\Delta \phi_{r}$ & $\frac{\Delta a_{r}}{a_{r}}$ & $\Delta \phi_{r}$ & $\frac{\Delta a_{r}}{a_{r}}$ & $\Delta \phi_{r}$ \\
\hline \hline-5 & -5 & $-4.81 \pm 0.82$ & $-5.28 \pm 0.86$ & +5 & -5 & $+4.47 \pm 0.88$ & $-5.49 \pm 0.85$ \\
& -2 & $-3.79 \pm 0.84$ & $-1.77 \pm 0.81$ & & -2 & $+7.16 \pm 0.91$ & $-2.43 \pm 0.89$ \\
& -1 & $-5.26 \pm 0.89$ & $-0.35 \pm 0.85$ & & -1 & $+5.31 \pm 0.89$ & $-1.84 \pm 0.86$ \\
& 0 & $-5.25 \pm 0.81$ & $-0.21 \pm 0.80$ & & 0 & $+4.82 \pm 0.90$ & $-1.16 \pm 0.87$ \\
& +1 & $-4.68 \pm 0.85$ & $1.07 \pm 0.87$ & & +1 & $+6.09 \pm 0.90$ & $1.75 \pm 0.85$ \\
& +2 & $-4.16 \pm 0.83$ & $1.79 \pm 0.86$ & & +2 & $+5.57 \pm 0.89$ & $1.75 \pm 0.87$ \\
& +5 & $-6.64 \pm 0.84$ & $4.15 \pm 0.87$ & & +5 & $+5.82 \pm 0.89$ & $4.63 \pm 0.89$ \\
\hline-2 & -5 & $-2.48 \pm 0.82$ & $-5.66 \pm 0.86$ & +2 & -5 & $+3.60 \pm 0.87$ & $-5.04 \pm 0.85$ \\
& -2 & $-0.11 \pm 0.83$ & $-2.32 \pm 0.88$ & & -2 & $+2.83 \pm 0.88$ & $-0.77 \pm 0.81$ \\
& -1 & $-2.39 \pm 0.88$ & $-2.10 \pm 0.86$ & & -1 & $+0.69 \pm 0.86$ & $-2.30 \pm 0.84$ \\
& 0 & $-1.70 \pm 0.80$ & $0.77 \pm 0.89$ & & 0 & $+3.51 \pm 0.83$ & $0.36 \pm 0.88$ \\
& +1 & $-0.67 \pm 0.88$ & $0.77 \pm 0.87$ & & +1 & $+0.64 \pm 0.85$ & $1.33 \pm 0.82$ \\
& +2 & $-2.61 \pm 0.87$ & $1.63 \pm 0.82$ & & +2 & $+3.17 \pm 0.80$ & $2.91 \pm 0.87$ \\
& +5 & $-3.05 \pm 0.84$ & $5.52 \pm 0.85$ & & +5 & $+2.73 \pm 0.83$ & $4.95 \pm 0.86$ \\
\hline-1 & -5 & $-0.47 \pm 0.81$ & $-5.84 \pm 0.89$ & +1 & -5 & $+0.73 \pm 0.87$ & $-6.23 \pm 0.87$ \\
& -2 & $-1.97 \pm 0.88$ & $-2.29 \pm 0.88$ & & -2 & $+2.89 \pm 0.83$ & $-2.13 \pm 0.84$ \\
& -1 & $-2.01 \pm 0.85$ & $-2.14 \pm 0.88$ & & -1 & $+0.81 \pm 0.88$ & $-1.96 \pm 0.86$ \\
& 0 & $-1.45 \pm 0.88$ & $0.60 \pm 0.87$ & & 0 & $+0.02 \pm 0.80$ & $-0.67 \pm 0.88$ \\
& +1 & $+0.24 \pm 0.85$ & $2.15 \pm 0.88$ & & +1 & $+1.27 \pm 0.80$ & $1.16 \pm 0.87$ \\
& +2 & $-2.23 \pm 0.84$ & $0.02 \pm 0.86$ & & +2 & $+0.17 \pm 0.89$ & $2.73 \pm 0.86$ \\
+5 & $-1.48 \pm 0.88$ & $6.78 \pm 0.88$ & & +5 & $+1.67 \pm 0.81$ & $4.09 \pm 0.87$ \\
\hline 0 & -5 & $+1.00 \pm 0.86$ & $-4.73 \pm 0.82$ & 0 & +5 & $-0.91 \pm 0.84$ & $3.23 \pm 0.89$ \\
& -2 & $+0.52 \pm 0.83$ & $-2.91 \pm 0.87$ & & +2 & $+1.19 \pm 0.81$ & $2.05 \pm 0.86$ \\
& -1 & $-0.66 \pm 0.86$ & $0.47 \pm 0.87$ & & +1 & $-1.73 \pm 0.85$ & $2.29 \pm 0.85$ \\
0 & $-0.84 \pm 0.81$ & $0.37 \pm 0.86$ & & & & \\
\hline
\end{tabular}




\section{7 $\quad$ Systematic uncertainty in simulated data}

Though eventually the systematic uncertainty in our measurements will depend on the measured values of asymmetry parameters from data, we have made several preliminary studies to quantify approximately the level of systematic uncertainty we expect.

\subsubsection{Experimental and model-dependent systematics}

Systematic uncertainties in quantities describing $C P$ asymmetry arise from experimental effects, and also (in case of the model-dependent analysis) from uncertainties in the nature of the models used to describe the Dalitz plot structure. We determine these separately and add them in quadrature. In both cases, we assign the maximum deviation in the observed quantities (i.e., $\Delta a_{r} / a_{r}, \Delta \phi_{r}$, and $\chi^{2} / \nu$ ) from the central value as a systematic uncertainty, taking correlations among fit-parameters (or moment distributions in case of the model-independent analysis) into account.

We characterize the following types of uncertainties as model dependent:

- uncertainties due to mass-width values of the resonances and damping factor used to describe the Dalitz plot amplitudes. We estimate this from the spread in amplitudes when either of the resonance parameters are changed by one standard deviation $(\sigma)$.

- uncertainties in lineshape of the intermediate states, e.g., $K \pi S$-wave and $f_{0} / a_{0}(980)$. We estimate this uncertainty by trying alternative lineshapes.

- in case of $D^{0} / \bar{D}^{0} \rightarrow K^{-} K^{+} \pi^{0}$, there are two models to describe the Standard Model Dalitz plot distribution. We use Model-I for our analysis, and estimate the systematic uncertainty due to this by using Model-II instead.

Similarly, we estimate the experimental uncertainty from the variation in results when

- either the signal efficiency parameters for $D^{0}$ and $\bar{D}^{0}$ events are varied by $1 \sigma$ in each case, or

- the background shape is varied.

- We also repeat the measurements using the same efficiency function and background shape for both $D^{0}$ and $\bar{D}^{0}$ events and take the shift in central values as another experimental systematic uncertainty.

- Another experimental systematic uncertainty due to $D^{0} / \bar{D}^{0}$ mistagging in our data sample is described below.

\subsubsection{Systematic uncertainty from $D^{0} / \bar{D}^{0}$ mistagging}

Unlike in two-body $\left(D^{0} \rightarrow K^{-} K^{+}, \pi^{-} \pi^{+}\right)$analysis, here we are trying to measure asymmetry in the underlying amplitudes and phases of the interfering intermediate resonances with almost equal numbers of $D^{0}$ and $\bar{D}^{0}$ events. Now, some of the $D^{0}$ 
events may be misidentified as $\bar{D}^{0}$ or vice versa which will change the Dalitz plot interference structure somewhat. But this change is rather small and systematic uncertainty due to misidentification is a second-order effect. Below we tabulate the results of our studies of this systematic effect using simulation for different levels of mistagging.

Table 7.10: Observed asymmetry in amplitude and phase and the $\chi^{2} /$ dof values for different levels of cross-feed. In the model-dependent fit, the amplitude and phase coefficients are determined using $D^{0} \rightarrow \rho^{+} \pi^{-}\left(\right.$for $D^{0} \rightarrow \pi^{-} \pi^{+} \pi^{0}$ ) and $D^{0} \rightarrow$ $K^{*+} K^{-}$(for $D^{0} \rightarrow K^{-} K^{+} \pi^{0}$ ) decay.

\begin{tabular}{|r|r|r|r|r|r|r|}
\hline Cross-feed (\%) & $\Delta a_{\rho^{0}} / a_{\rho^{0}}(\%)$ & $\Delta \phi_{\rho^{0}}\left(^{\circ}\right)$ & $\chi^{2} / \nu$ & $\Delta a_{\phi} / a_{\phi}(\%)$ & $\Delta \phi_{\phi}\left(^{\circ}\right)$ & $\chi^{2} / \nu$ \\
\hline \hline 0.1 & 0.02 & 0.03 & 0.99 & 0.01 & 0.05 & 1.02 \\
0.2 & 0.16 & 0.12 & 1.03 & 0.05 & 0.13 & 1.01 \\
0.4 & 0.21 & 0.19 & 1.04 & 0.12 & 0.45 & 1.04 \\
0.5 & 0.28 & 0.32 & 1.07 & 0.15 & 0.47 & 1.05 \\
0.8 & 0.36 & 0.84 & 1.10 & 0.24 & 1.21 & 1.11 \\
1.0 & 0.44 & 1.12 & 1.09 & 0.41 & 1.68 & 1.10 \\
\hline
\end{tabular}

\subsection{Validation study using simulation treated as data}

In order to validate the methodology proposed in this document for establishing $C P$ violation and for extracting the corresponding asymmetry parameters, we perform the whole analysis on simulated event sample of the size of our present dataset. We perform this validation study for two extreme cases: (a) no $C P$ violation, and (b) $5 \%$ $C P$ violation. The statistical uncertainties in the $\chi^{2} / \nu$ values for model-independent analysis have been taken from Fig. 7.7 (for angular moments distributions) and Fig. 7.9 (for Dalitz plot distributions). The expectations for the null hypothesis (no $C P$ violation) in the two model-independent analysis methods are also summarized in Table 7.11.

\begin{tabular}{l|r|r|r}
\hline \hline Mode & $\chi^{2} / \nu:$ Dalitz plot & $\chi^{2} / \nu: h^{+} h^{-}$moments & $\chi^{2} / \nu: h^{+} \pi^{0}$ moments \\
\hline$\pi^{-} \pi^{+} \pi^{0}$ & $1.012 \pm 0.018$ & $1.032 \pm 0.026$ & $1.027 \pm 0.021$ \\
$K^{-} K^{+} \pi^{0}$ & $1.021 \pm 0.036$ & $1.019 \pm 0.033$ & $1.017 \pm 0.028$ \\
\hline \hline
\end{tabular}

Table 7.11: The values and spread of $\chi^{2} / \nu$ for the null hypothesis (no $C P$ violation) in the two model-independent analysis methods. These were obtained using 500 simulation experiments. See Fig. 7.7 (for angular moments distributions) and Fig. 7.9 (for Dalitz plot distributions) for further details.

\subsubsection{Analysis with $C P$-symmetric $D \rightarrow \pi^{-} \pi^{+} \pi^{0}$ simulated events}

We perform the analysis on a simulated data sample in which both $D^{0}$ and $\bar{D}^{0}$ events have exactly the same amplitudes and phases. The size of the sample is 
roughly equal to the size of our present dataset. The results of the analysis and confidence levels (CL) for for consistency with no $C P$ asymmetry are summarized below.

$$
\begin{aligned}
& \chi^{2} / \nu(\text { Dalitz })=1.051 \pm 0.018 \text { (stat.) } \pm 0.011 \text { (syst.) }[\nu=1429] \\
& \text { CL for consistency with no } C P \text { violation }=\frac{1}{2} \cdot \operatorname{Erfc}\left(\frac{1.051-1.012}{\sqrt{2} \times 0.018}\right)=1.51 \% . \\
& \chi^{2} / \nu\left(\pi^{+} \pi^{-} \text {moments) }=1.069 \pm 0.026 \text { (stat.) } \pm 0.015 \text { (syst.) }[\nu=320]\right. \\
& \text { CL for consistency with no } C P \text { violation }=\frac{1}{2} . \operatorname{Erfc}\left(\frac{1.069-1.032}{\sqrt{2} \times 0.026}\right)=7.74 \% . \\
& \frac{\Delta a_{\rho^{0}}}{a_{\rho^{0}}}=0.0027 \pm 0.0089 \text { (stat.) } \pm 0.0036 \text { (syst.) } \\
& \Delta \phi_{\rho^{0}}=0.28^{\circ} \pm 0.72^{\circ} \text { (stat.) } \pm 0.35^{\circ} \text { (syst.) } \\
& \frac{\Delta a_{\rho^{+}}}{a_{\rho^{+}}}=0.0018 \pm 0.0047 \text { (stat.) } \pm 0.0019 \text { (syst.) } \\
& \Delta \phi_{\rho^{+}}=0.23^{\circ} \pm 0.43^{\circ} \text { (stat.) } \pm 0.20^{\circ} \text { (syst.) }
\end{aligned}
$$

\subsubsection{Analysis with asymmetric $D \rightarrow \pi^{-} \pi^{+} \pi^{0}$ simulated events}

We perform the analysis on a simulated data sample in which $\bar{D}^{0} \rightarrow \rho^{0} \pi^{0}$ have $5 \%$ larger amplitude and $5^{\circ}$ larger phase compared to those of $D^{0} \rightarrow \rho^{0} \pi^{0}$. The size of this sample is roughly equal to the size of our present dataset. The results of the analysis are summarized below.

$\chi^{2} / \nu($ Dalitz $)=1.389 \pm 0.018$ (stat.) \pm 0.016 (syst.) $[\nu=1429]$

CL for consistency with no $C P$ violation $=\frac{1}{2} \cdot \operatorname{Erfc}\left(\frac{1.389-1.012}{\sqrt{2} \times 0.018}\right)=0.00 \%$.

$\chi^{2} / \nu\left(\pi^{+} \pi^{-}\right.$moments $)=1.654 \pm 0.026$ (stat.) \pm 0.021 (syst.) $[\nu=320]$

CL for consistency with no $C P$ violation $=\frac{1}{2} \cdot \operatorname{Erfc}\left(\frac{1.654-1.032}{\sqrt{2} \times 0.026}\right)=0.00 \%$.

$$
\begin{aligned}
& \frac{\Delta a_{\rho^{0}}}{a_{\rho^{0}}}=0.0478 \pm 0.0084 \text { (stat.) } \pm 0.0045 \text { (syst.) } \\
& \Delta \phi_{\rho^{0}}=5.16^{\circ} \pm 0.77^{\circ} \text { (stat.) } \pm 0.40^{\circ} \text { (syst.) } \\
& \frac{\Delta a_{\rho^{+}}}{a_{\rho^{+}}}=0.0024 \pm 0.0044 \text { (stat.) } \pm 0.0023 \text { (syst.) } \\
& \Delta \phi_{\rho^{+}}=0.21^{\circ} \pm 0.40^{\circ} \text { (stat.) } \pm 0.22^{\circ} \text { (syst.) }
\end{aligned}
$$

\subsubsection{Analysis with $C P$-symmetric $D \rightarrow K^{-} K^{+} \pi^{0}$ simulated events}

We perform the analysis on a simulated data sample in which both $D^{0}$ and $\bar{D}^{0}$ events have exactly the same amplitudes and phases. The size of the sample is roughly equal to the size of our present dataset. The results of the analysis are summarized below.

$$
\begin{aligned}
& \chi^{2} / \nu(\text { Dalitz })=1.036 \pm 0.036 \text { (stat.) } \pm 0.043 \text { (syst.) }[\nu=726] \\
& \text { CL for consistency with no } C P \text { violation }=\frac{1}{2} \cdot \operatorname{Erfc}\left(\frac{1.036-1.021}{\sqrt{2} \times 0.036}\right)=33.85 \% . \\
& \chi^{2} / \nu\left(K^{+} K^{-} \text {moments }\right)=1.045 \pm 0.033 \text { (stat.) } \pm 0.092 \text { (syst.) }[\nu=160] \\
& \text { CL for consistency with no } C P \text { violation }=\frac{1}{2} \cdot \operatorname{Erfc}\left(\frac{1.045-1.019}{\sqrt{2} \times 0.033}\right)=21.54 \% .
\end{aligned}
$$




$$
\begin{aligned}
& \frac{\Delta a_{\phi}}{a_{\phi}}=0.0038 \pm 0.0114 \text { (stat.) } \pm 0.0081 \text { (syst.) } \\
& \Delta \phi_{\phi}=0.76^{\circ} \pm 1.20^{\circ} \text { (stat.) } \pm 1.22^{\circ} \text { (syst.) } \\
& \frac{\Delta a_{K^{*+}}}{a_{\rho^{+}}}=-0.0082 \pm 0.0084 \text { (stat.) } \pm 0.0047 \text { (syst.) } \\
& \Delta \phi_{K^{*+}}=0.33^{\circ} \pm 0.87^{\circ} \text { (stat.) } \pm 0.36^{\circ} \text { (syst.) }
\end{aligned}
$$

\subsubsection{Analysis with asymmetric $D \rightarrow K^{-} K^{+} \pi^{0}$ simulated events}

We perform the analysis on a simulated data sample in which $\bar{D}^{0} \rightarrow \phi(1020) \pi^{0}$ have $5 \%$ larger amplitude and $5^{\circ}$ larger phase compared to those of $D^{0} \rightarrow \phi(1020) \pi^{0}$. The size of the sample is roughly equal to the size of our present dataset. The results of the analysis are summarized below.

$$
\begin{aligned}
& \chi^{2} / \nu(\text { Dalitz) }=1.293 \pm 0.036 \text { (stat.) } \pm 0.059 \text { (syst.) }[\nu=726] \\
& \text { CL for consistency with no } C P \text { violation }=\frac{1}{2} . \operatorname{Erfc}\left(\frac{1.293-1.021}{\sqrt{2} \times 0.036}\right)=0.00 \% . \\
& \chi^{2} / \nu\left(K^{+} K^{-} \text {moments) }=1.406 \pm 0.033 \text { (stat.) } \pm 0.124 \text { (syst.) }[\nu=160]\right. \\
& \text { CL for consistency with no } C P \text { violation }=\frac{1}{2} \text {. Erfc }\left(\frac{1.406-1.019}{\sqrt{2} \times 0.033}\right)=0.00 \% . \\
& \frac{\Delta a_{\phi}}{a_{\phi}}=0.0641 \pm 0.0116 \text { (stat.) } \pm 0.0087 \text { (syst.) } \\
& \Delta \phi_{\phi}=3.98^{\circ} \pm 1.73^{\circ} \text { (stat.) } \pm 1.28^{\circ} \text { (syst.) } \\
& \frac{\Delta a_{K^{*+}}}{a_{K^{*+}}}=-0.0074 \pm 0.0082 \text { (stat.) } \pm 0.0038 \text { (syst.) } \\
& \Delta \phi_{K^{*+}}=0.45^{\circ} \pm 0.82^{\circ} \text { (stat.) } \pm 0.44^{\circ} \text { (syst.) }
\end{aligned}
$$

\subsubsection{Validation of the model-independent method on data}

Since we obtained the correlation between the angular moments distributions using simulation, we perform a validation study of the method on real data and describe the results here. Being still blind to the charm-flavor of the $D^{0}$ candidate, we divide the $D^{0} / \bar{D}^{0} \rightarrow \pi^{-} \pi^{+} \pi^{0}$ data sample into two categories by randomly assigning the events. We repeat this procedure 500 times. Since there is an almost infinite number of ways to to randomly assign $\sim 100000$ events into two categories of roughly equal size, the 500 samples obtained by this procedure are quasi-independent.

Now, we perform the model-independent (moments method) analysis on all these samples and plot the resulting $\chi^{2} / \nu$ distributions. These are shown in Fig. 7.10. The results obtained are consistent with the no $C P$-violation hypothesis.

\subsubsection{Validation on full available simulation sample}

Till now, we have performed the validation study of our methods to measure $C P$ asymmetry using simulated events treating them as data. In order to obtain $C P$ violating sample from a $C P$-symmetric simulation sample, we had to throw away lots of events. So, to keep the procedure simple, we used exactly the same size datasets for both $C P$-symmetric and $C P$-violating samples in the validation process (this size being same as the size of our real data). 

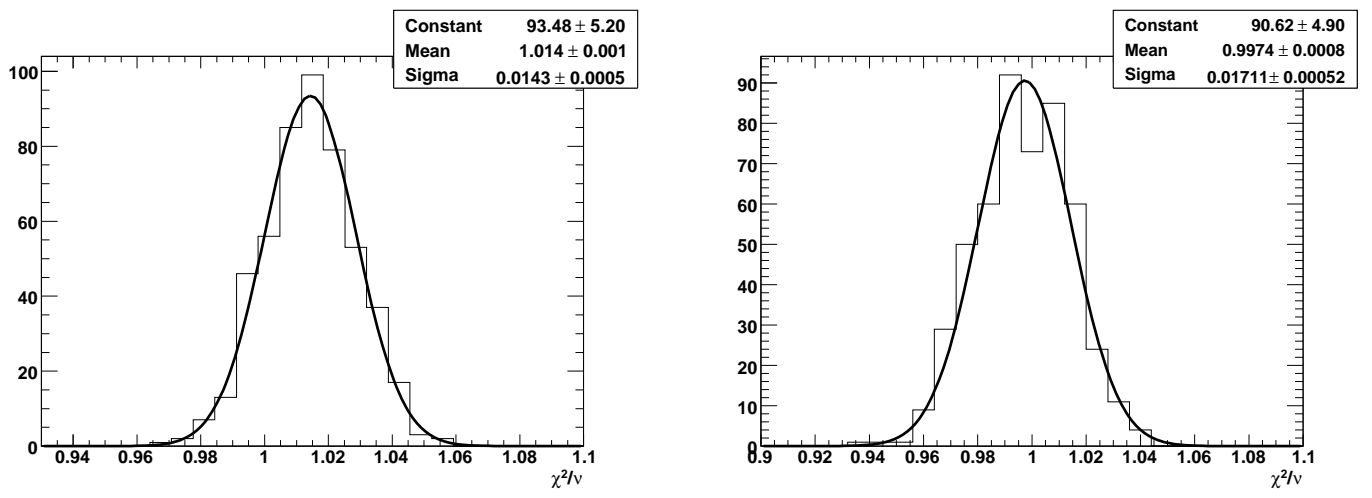

Figure 7.10: The spread of $\chi^{2} / \nu$ values corresponding to the difference in angular moments distributions between two catagories of randomly chosen data events: $\pi^{+} \pi^{-}$ (left) and $\pi^{+} \pi^{0}$ (right).

However, for the $C P$-symmetric case, we can also use all the available simulation dataset $\left(\sim 675 \mathrm{fb}^{-1}\right.$ of $\pi^{-} \pi^{+} \pi^{0}$ and $\sim 440 \mathrm{fb}^{-1}$ of $\left.K^{-} K^{+} \pi^{0}\right)$ and repeat the validation exercise. If the analysis of this dataset yields no $C P$ asymmetry at reasonable confidence levels, we will gain further confidence in our procedure.

The results of this analysis are presented below.

For the $\pi^{-} \pi^{+} \pi^{0}$ final state:

$\chi^{2} / \nu($ Dalitz $)=1.017 \pm 0.018$ (stat.)

Confidence Level for consistency with no $C P$ violation $=39.06 \%$.

$\chi^{2} / \nu\left(\pi^{+} \pi^{-}\right.$moments $)=1.040 \pm 0.026$ (stat.)

Confidence Level for consistency with no $C P$ violation $=37.92 \%$.

$\chi^{2} / \nu\left(\pi^{+} \pi^{0}\right.$ moments $)=1.032 \pm 0.021$ (stat.)

Confidence Level for consistency with no $C P$ violation $=40.59 \%$.

Table 7.12: The results obtained from the model-dependent analysis of $D^{0} / \bar{D}^{0} \rightarrow$ $\pi^{-} \pi^{+} \pi^{0}$ Dalitz plot. We define amplitude coefficients, $a_{r}$ and $\phi_{r}$, relative to those of the $\rho(770)^{+}$. The errors are statistical.

\begin{tabular}{l|r|r|r}
\hline State & $a_{r}^{\bar{D}^{0}}-a_{r}^{D^{0}}(\%)$ & $\phi_{r}^{\bar{D}^{0}}-\phi_{r}^{D^{0}}\left({ }^{\circ}\right)$ & $f_{r}^{\bar{D}^{0}}-f_{r}^{D^{0}}(\%)$ \\
\hline$\rho^{+}(770)$ & 0. (fixed) & 0. (fixed) & $0.05 \pm 0.32$ \\
$\rho^{0}(770)$ & $0.13 \pm 0.46$ & $-0.12 \pm 0.63$ & $0.18 \pm 0.59$ \\
$\rho^{-}(770)$ & $-0.06 \pm 0.53$ & $0.23 \pm 0.72$ & $-0.02 \pm 0.66$ \\
Non-Res & $1.7 \pm 3.8$ & $2 \pm 4$ & $0.08 \pm 0.27$ \\
\hline
\end{tabular}

For the $K^{-} K^{+} \pi^{0}$ final state: 
Table 7.13: The results obtained from the model-dependent analysis of $D^{0} / \bar{D}^{0} \rightarrow$ $K^{-} K^{+} \pi^{0}$ Dalitz plot. We define amplitude coefficients, $a_{r}$ and $\phi_{r}$, relative to those of the $K^{*}(892)^{+}$. The errors are statistical. We use Model-I of Ref. [21] here.

\begin{tabular}{l|r|r|r}
\hline State & $a_{r}^{\bar{D}^{0}}-a_{r}^{D^{0}}$ & $\phi_{r}^{\bar{D}^{0}}-\phi_{r}^{D^{0}}\left({ }^{\circ}\right)$ & $f_{r}^{\bar{D}^{0}}-f_{r}^{D^{0}}(\%)$ \\
\hline$K^{*}(892)^{+}$ & 0. (fixed) & 0. (fixed) & $0.2 \pm 1.1$ \\
$K^{*}(1410)^{+}$ & $0.21 \pm 0.58$ & $3 \pm 13$ & $-0.8 \pm 1.8$ \\
$K^{+} \pi^{0}(S)$ & $-0.22 \pm 0.48$ & $5 \pm 24$ & $1.9 \pm 4.2$ \\
$\phi(1020)$ & $-0.01 \pm 0.02$ & $8 \pm 18$ & $0.0 \pm 1.0$ \\
$f_{0}(980)$ & $0.4 \pm 1.1$ & $-10 \pm 17$ & $-0.5 \pm 0.8$ \\
$f_{2}^{\prime}(1525)$ & $-0.11 \pm 0.50$ & $20 \pm 25$ & $0.05 \pm 0.06$ \\
$K^{*}(892)^{-}$ & $0.01 \pm 0.02$ & $-1.0 \pm 2.2$ & $0.2 \pm 1.0$ \\
$K^{*}(1410)^{-}$ & $-0.31 \pm 0.76$ & $-8 \pm 10$ & $2.1 \pm 2.3$ \\
$K^{-} \pi^{0}(S)$ & $0.06 \pm 0.44$ & $5 \pm 30$ & $0.3 \pm 2.1$ \\
\hline
\end{tabular}

$\chi^{2} / \nu($ Dalitz $)=1.014 \pm 0.036$ (stat.)

Confidence Level for consistency with no $C P$ violation $=57.71 \%$.

$\chi^{2} / \nu\left(K^{+} K^{-}\right.$moments $)=1.022 \pm 0.033$ (stat.)

Confidence Level for consistency with no $C P$ violation $=46.38 \%$.

$\chi^{2} / \nu\left(K^{+} \pi^{0}\right.$ moments $)=1.013 \pm 0.028$ (stat.)

Confidence Level for consistency with no $C P$ violation $=55.68 \%$.

\subsection{Asymmetry in Dalitz plot}

It is worth noting that the three methods to determine $C P$ asymmetry explained earlier are complementary to each other.

- The $\chi^{2} / \nu$ value obtained from the difference in 2-dimensional scatter Dalitz plot distribution tells us whether there is an asymmetry anywhere in the Dalitz plot or not. But it does not tell much about the amount of asymmetry or in which two-body channel the source of asymmetry is located.

- The $\chi^{2} / \nu$ value obtained from the difference in angular moments distribution tells us in which two-body channel the source of asymmetry is located. But it does not tell much about the amount of asymmetry.

- The model-dependent "isobar model" fit can tell us about the amount of asymmetry as well as the source of asymmetry down to a specific intermdediate state (or set of intermdediate states). The only drawback with this method, apart from the model-dependence, is the fact that there may be a discrete ambiguity. This ambiguity can be resolved either by parametrizing the Dalitz plot in an alternative way or with the help of the model-independent $\chi^{2} / \nu$ value obtained from the difference in angular moment distributions. We will follow both these approaches. 


\subsection{Asymmetry in branching ratio}

So far in this document we have talked about measuring direct $C P$-asymmetry in neutral $D$ meson decays by using the difference in the Dalitz plot structures of $D^{0}$ and $\bar{D}^{0}$. We note, however, that any difference in the overall signal branching fraction (integrated over the entire phase space available) between $D^{0}$ and $\bar{D}^{0}$ decays to the $\pi^{-} \pi^{+} \pi^{0}, K^{-} K^{+} \pi^{0}$ final states will also indicate a direct $C P$-asymmetry in charmed meson decays. This information is not captured by the difference in the Dalitz plot structures, and is complementary to the full Dalitz plot analysis. To incorporate all the available information in our data, we also look for asymmetry in the signal yield of the $D^{0}$ and $\bar{D}^{0}$ decays to these three-body final states.

The procedure to extract signal yield and efficiency corrections and the estimation of statistical and systematic uncertainties is described in Chapter 3 . With $385 \mathrm{fb}^{-1}$, we propose to repeat the same procedure and measure the asymmetries

$$
\begin{gathered}
a_{C P}^{\pi \pi \pi^{0}}=\frac{\Gamma\left(D^{0} \rightarrow \pi^{-} \pi^{+} \pi^{0}\right)-\Gamma\left(\bar{D}^{0} \rightarrow \pi^{+} \pi^{-} \pi^{0}\right)}{\Gamma\left(D^{0} \rightarrow \pi^{-} \pi^{+} \pi^{0}\right)+\Gamma\left(\bar{D}^{0} \rightarrow \pi^{+} \pi^{-} \pi^{0}\right)} \\
a_{C P}^{K K \pi^{0}}=\frac{\Gamma\left(D^{0} \rightarrow K^{-} K^{+} \pi^{0}\right)-\Gamma\left(\bar{D}^{0} \rightarrow K^{+} K^{-} \pi^{0}\right)}{\Gamma\left(D^{0} \rightarrow K^{-} K^{+} \pi^{0}\right)+\Gamma\left(\bar{D}^{0} \rightarrow K^{+} K^{-} \pi^{0}\right)} .
\end{gathered}
$$

In order to be able to calculate $C P$ asymmetry in the signal yields, we must take into account the forward-backward (FB) asymmetry in $c \bar{c}$ production which causes an integrated asymmetry between the reconstruction of $D^{0}$ and $\bar{D}^{0}$ decays at the centerof-mass energies of around $10.6 \mathrm{GeV}$. The production asymmetry has two physical components: (1.) the interference in $e^{+} e^{-} \rightarrow c \bar{c}$ production between the processes mediated by a virtual $\gamma$ and by a virtual $Z^{0}$, and (2.) higher-order QED effects having polar angle dependence, which may peak sharply in the forward or backward directions. For precise quantification of this asymmetry in $D^{0}$-flavor assignment

(tagging), we use the relative soft pion efficiency map, i.e., $\frac{\epsilon_{\pi_{s}^{+}}}{\epsilon_{\pi_{s}^{-}}}$as function of the soft pion momentum-magnitude and polar angle. The azimuthal angle is found to be uncorrelated, and is therefore sufficiently treated as an integrated scale factor since charm production is azimuthally uniform. Fig. 7.11 shows this efficiency map for data events. The uncertainties shown here are due to the statistical uncertainties in the sample yields. After being weighted with this $\pi_{s}$ map to correct for soft-pion tagging asymmetry, the signal modes yields (with remaining production asymmetries) can be analyzed for evidence of direct $C P$ violation.

\subsection{Steps for unblinding}

- Step 1: Repeat the procedure of chapter 3 to obtain the asymmetry in signal yields after applying $\pi_{s}$ efficiency corrections.

- Step 2: Separate out the asymmetry in the branching ratio from the asymmetry in the Dalitz plot structure. For this purpose, directly compare the Dalitz plot distributions of $D^{0}$ and $\bar{D}^{0}$ events after normalizing them by the number of events, and obtain $\chi^{2} / \nu$ value using exactly the same binning and procedure 

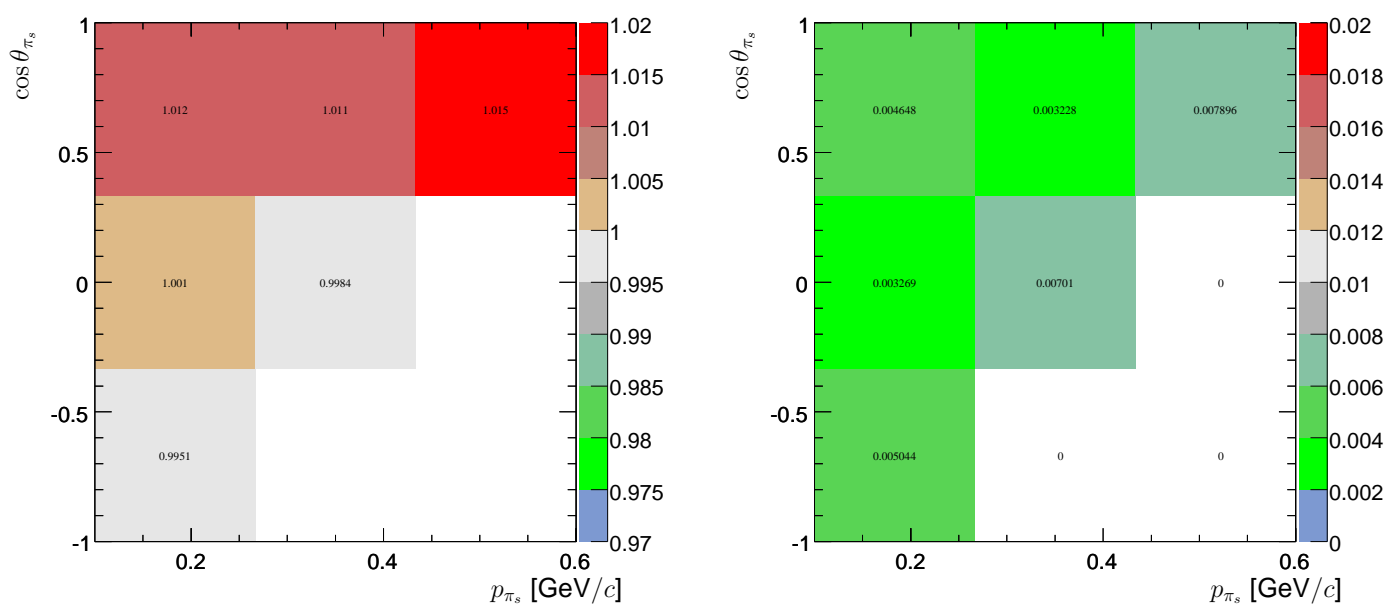

Figure 7.11: Relative $\pi_{s}$ efficiency map (left), $\frac{\epsilon_{\pi_{s}^{+}}}{\epsilon_{\pi_{s}^{-}}}$, with statistical errors (right).

as used in the validation studies.

$$
\chi^{2}=\sum_{\text {bins }} \Delta^{2}
$$

where

$$
\Delta=\frac{n_{\bar{D}^{0}}-R \cdot n_{D^{0}}}{\sqrt{\sigma_{n_{\bar{D}^{0}}}^{2}+R^{2} \cdot \sigma_{n_{D^{0}}}^{2}}} .
$$

Here $n_{D^{0}}$ denotes the number of $D^{0}$ events, $n_{\bar{D}^{0}}$ the number of $\bar{D}^{0}$ events in a bin, and $\sigma$ represents the uncertainty in a number. The quantity $R$ is the ratio of the total number of $\bar{D}^{0}$ events integrated over the entire phase space to the integrated total number of $D^{0}$ events.

- Step 3: Plot the difference in first eight angular moments $(l=0-7)$ between $D^{0}$ and $\bar{D}^{0}$ events and obtain a combined $\chi^{2} / \nu$ value, following the procedure used in the validation studies.

$$
\chi^{2}=\sum_{b i n s} \sum_{i} \sum_{j} X_{i} \rho_{i j} X_{j}
$$

where

$$
X_{i}=\frac{P_{i}^{\bar{D}^{0}}-R \cdot P_{i}^{D^{0}}}{\sqrt{\sigma_{P_{i}^{D^{0}}}^{2}+R^{2} \cdot \sigma_{P_{i}^{D^{0}}}^{2}}} .
$$

Here $P_{i}$ and $P_{j}$ are Legendre polynomial moments of order $i$ and $j$ respectively, $\rho_{i j}$ is the correlation coefficient between the difference in pair of moments of orders $i$ and $j$ as defined in Eq. 7.8, and $R$ is the ratio of the total number of $\bar{D}^{0}$ events integrated over the entire phase space to the integrated total number of $D^{0}$ events. 
- Step 4: Perform the Dalitz plot fit for $D^{0}$ and $\bar{D}^{0}$ events and report the asymmetry in the dominant intermediate states.

- Step 5: Repeat the Dalitz plot fit, this time using a different resonant state as the reference [e.g., $\rho(770)^{0}$ instead of $\rho(770)^{+}$]. This may help in pinpointing the source of any observed asymmetry, as explained in detail in Chapter 9.

- Step 6: Finalize the evaluation of systematic uncertainties, including all the sources described earlier, and any new systematics we identify in the process.

\subsection{Results from data}

\subsubsection{Asymmetry in Dalitz plot distribution}

A direct comparison of the efficiency-corrected and background-subtracted Dalitz plots for $D^{0}$ and $\bar{D}^{0}$ events is the simplest way to look for $C P$ asymmetry. Figures 7.12-7.13 show the Dalitz plots for $D^{0}$ and $\bar{D}^{0}$ events and Fig. 7.14 shows their difference. Figure 7.15 shows the two-dimensional normalized residuals, $\Delta$, in Dalitz plot area elements, where

$$
\Delta=\left(n_{\bar{D}^{0}}-R \cdot n_{D^{0}}\right) / \sqrt{\sigma_{n_{D^{0}}}^{2}+R^{2} \cdot \sigma_{n_{D^{0}}}^{2}},
$$

and $n$ and $\sigma$ denote, respectively, the number of events and its uncertainty in a Dalitz plot element. The factor $R$, equal to $0.983 \pm 0.006$ for $\pi^{-} \pi^{+} \pi^{0}$ and $1.020 \pm 0.016$ for $K^{-} K^{+} \pi^{0}$, is the ratio of the total number of $\bar{D}^{0}$ events to the total number of $D^{0}$ events. This factor is introduced since the total number of events in the $D^{0}$ and $\bar{D}^{0}$ Dalitz plots may differ because of production crosssection asymmetry and/or asymmetry in the branching fraction values for $D^{0}$ and $\bar{D}^{0}$ decay to the same final state (we extract the latter separately, as described below). We calculate $\chi^{2}=\left(\sum_{\text {element }} \Delta^{2}\right) / \nu$, where $\nu$ is the number of Dalitz plot elements: 1429 for $\pi^{-} \pi^{+} \pi^{0}$ and 726 for $K^{-} K^{+} \pi^{0}$. We find the $\chi^{2}$ for $\pi^{-} \pi^{+} \pi^{0}\left(K^{-} K^{+} \pi^{0}\right)$ to be 1.020 (1.056) where we expect it to be 1.012 (1.021) with an r.m.s. deviation of 0.018 (0.036) from simulation of no $C P V$. We then obtain a one-sided Gaussian confidence level (CL) for consistency with no CPV of $32.8 \%$ for $\pi^{-} \pi^{+} \pi^{0}$ and $16.6 \%$ for $K^{-} K^{+} \pi^{0}$. From statistical uncertainty only there is no evidence of $C P V$. Systematic uncertainties are relatively small and have not been included in the CL calculation. Including them will improve consistency with the no $C P V$ hypothesis.

\subsubsection{Asymmetry in angular moments distribution}

The angular moments of the cosine of the helicity angle of the $\widetilde{D}$ decay products reflect the spin and mass structure of intermediate resonant and non-resonant amplitudes [21]. We define the helicity angle $\theta_{H}$ for decays of the type $\widetilde{D} \rightarrow(r \rightarrow A B) C$ as the angle between the momentum of $A$ in the $A B$ rest frame and the momentum of $A B$ in $\widetilde{D}$ rest frame. The moments of $\cos \theta_{H}, Z_{l}\left(\cos \theta_{H}\right) \equiv Y_{l}^{0}(m)$, are defined as the efficiency-corrected invariant mass distributions of events when weighted by spherical harmonic functions $Y_{l}^{0}(m)=\sqrt{1 / 2 \pi} P_{l}(m)$. Here $P_{l}$ are the Legendre 

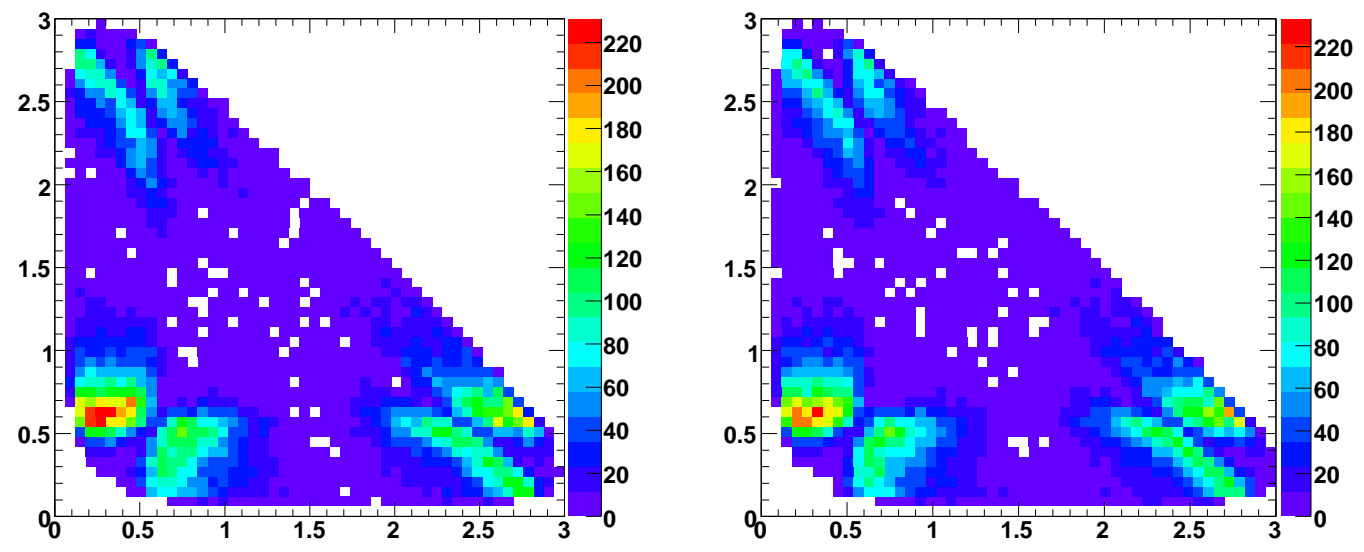

Figure 7.12: Dalitz plots for $D^{0}$ (left) and $\bar{D}^{0}$ (right) events in the $\widetilde{D} \rightarrow \pi^{-} \pi^{+} \pi^{0}$ decay.
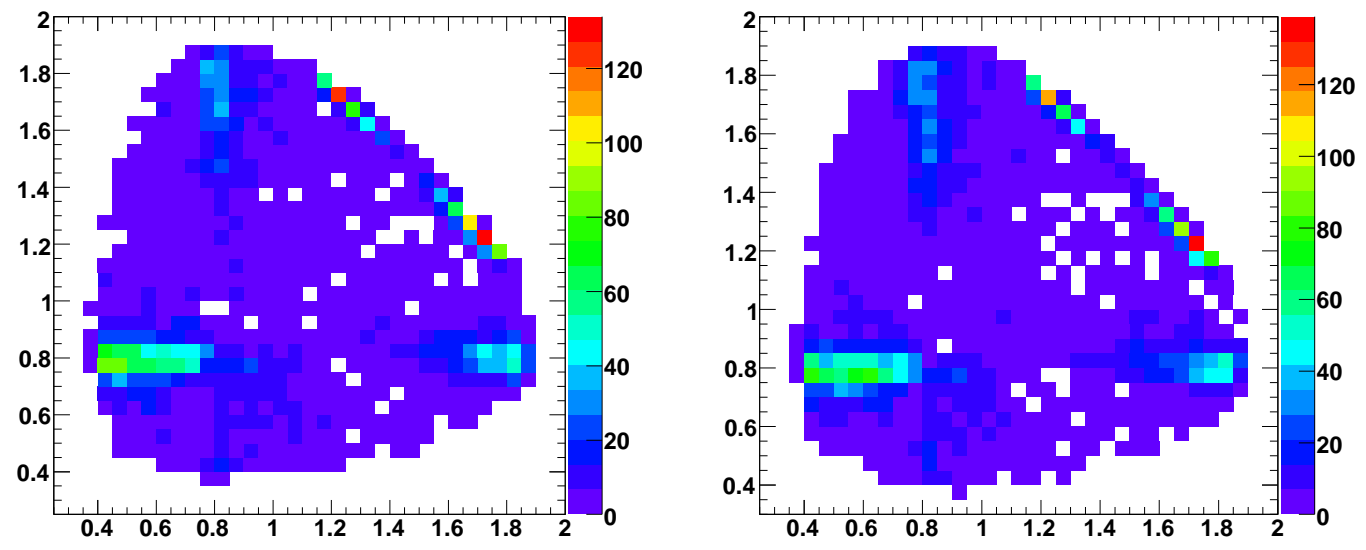

Figure 7.13: Dalitz plots for $D^{0}$ (left) and $\bar{D}^{0}$ (right) events in the $\widetilde{D} \rightarrow K^{-} K^{+} \pi^{0}$ decay. 

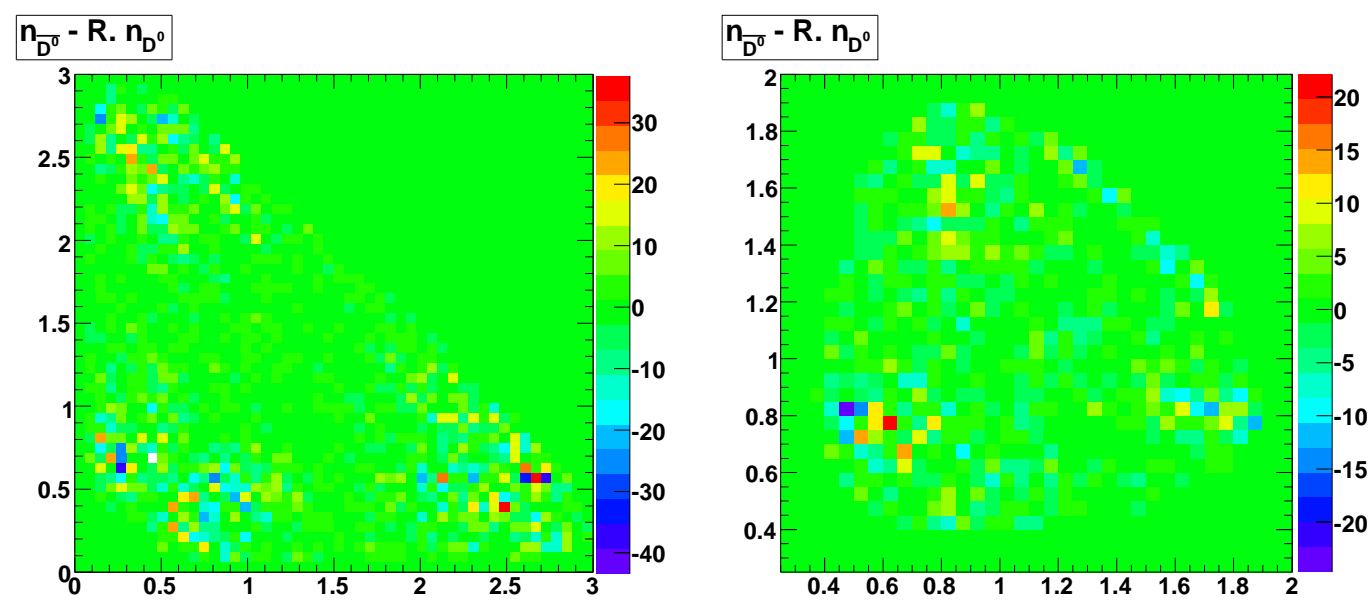

Figure 7.14: Difference between the $\bar{D}^{0}$ and $D^{0}$ Dalitz plots for $\widetilde{D} \rightarrow \pi^{-} \pi^{+} \pi^{0}$ (left) and $\widetilde{D} \rightarrow K^{-} K^{+} \pi^{0}$ (right).
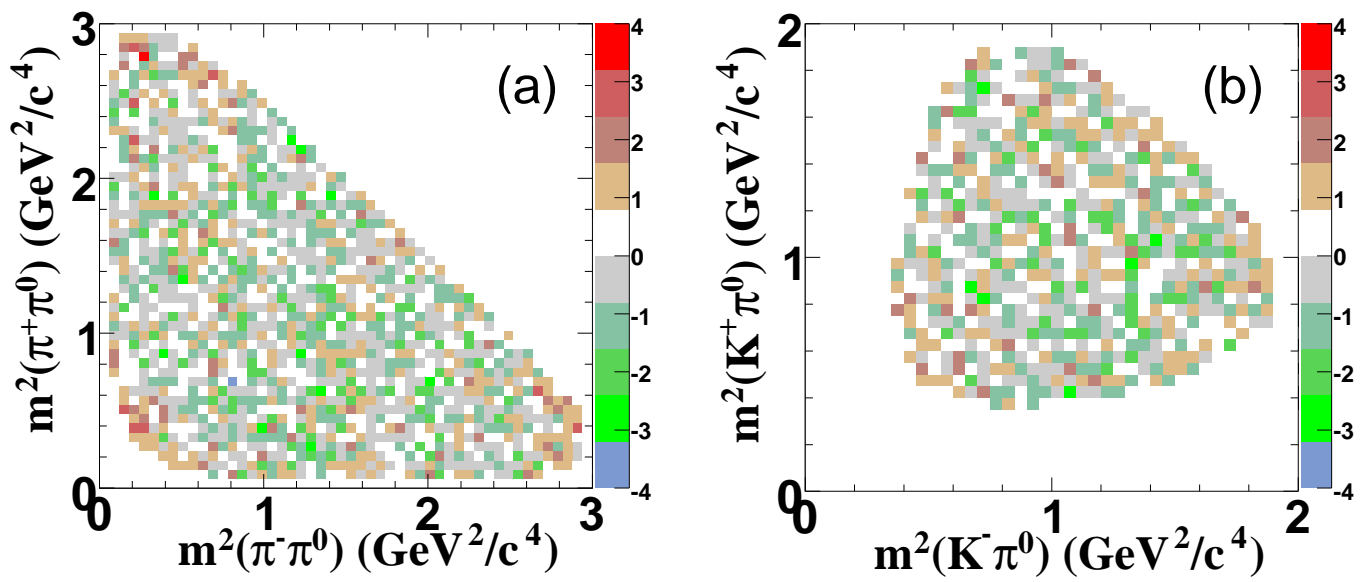

Figure 7.15: Normalized residuals in Dalitz plot elements, defined in Eq. 7.11, for (a) $\widetilde{D} \rightarrow \pi^{-} \pi^{+} \pi^{0}$ and (b) $\widetilde{D} \rightarrow K^{-} K^{+} \pi^{0}$. 

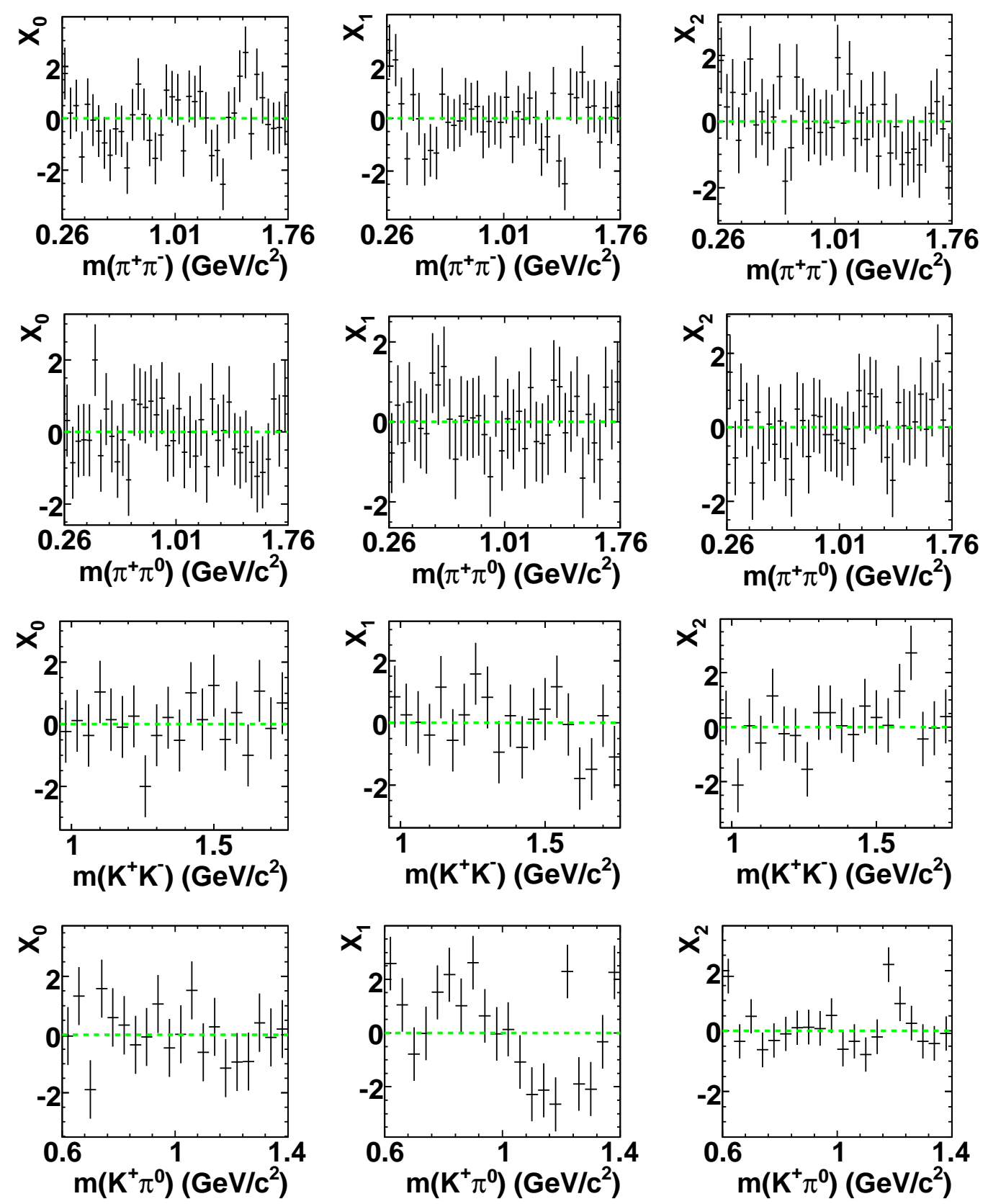

Figure 7.16: Normalized residuals for the first three Legendre polynomial moments of the $\pi^{-} \pi^{+}$(row 1 ), $\pi^{+} \pi^{0}$ (row 2), $K^{-} K^{+}$(row 3 ), and $K^{+} \pi^{0}$ (row 4) sub-systems. The confidence level for no $C P$ violation (dashed line) is obtained from the first eight moments. The error bars represent $\pm 1 \sigma$. 
polynomials of order $l$ normalized to 1 . To study differences between the $D^{0}$ and $\bar{D}^{0}$ decay amplitudes, we obtain the normalized differences in their moments [50], $P_{l}$ and $\overline{P_{l}}$, respectively, as:

$$
X_{l}=\left(\overline{P_{l}}-R \cdot P_{l}\right) / \sqrt{\sigma_{\overline{P_{l}}}^{2}+R^{2} \cdot \sigma_{P_{l}}^{2}},
$$

for $l=0-7$, as empirical observation shows that higher moments are vanishingly small. We show the $X_{l}$ distributions for the first three moments, i.e., $l=0-2$, in Fig. 7.16. We then calculate $\chi^{2}$ summed over all intervals in invariant mass as $\chi^{2}=\left(\sum_{0}^{k} \sum_{i=0}^{7} \sum_{j=0}^{7} X_{i} \rho_{i j} X_{j}\right) / 8 k$, where $k$ is the number of intervals and $\rho_{i j}$ is the correlation coefficient between $X_{i}, X_{j}$ :

$$
\rho_{i j}=\frac{\left\langle X_{i} X_{j}\right\rangle-\left\langle X_{i}\right\rangle\left\langle X_{j}\right\rangle}{\sqrt{\left\langle X_{i}^{2}\right\rangle-\left\langle X_{i}\right\rangle^{2}} \cdot \sqrt{\left\langle X_{j}^{2}\right\rangle-\left\langle X_{j}\right\rangle^{2}}} .
$$

We determine $\rho_{i j}$ in each of the relevant two-body invariant mass intervals by performing several hundred simulation experiments, generating $D^{0}$ and $\bar{D}^{0}$ events with no $C P V$. We validate the method on real data by randomly assigning the events, without defining the flavor of the $\widetilde{D}$ meson, into two disjoint categories and then calculating the $\chi^{2}$ for the difference in their angular moments. We repeat this experiment 500 times, each time assigning events randomly in different ways, and find the resulting $\chi^{2}$ distribution consistent with no $C P V$, as expected. We then define the $\widetilde{D}$ flavor in the data and calculate the $\chi^{2}$ values for the two-body channels with charge combinations,+- and,+ 0 . Finally, we obtain a one-sided Gaussian CL for consistency with no $C P V$ using the reference value and r.m.s. deviation from simulation. We find the CL for no $C P V$ to be $28.2 \%$ for the $\pi^{+} \pi^{-}, 28.4 \%$ for the $\pi^{+} \pi^{0}, 63.1 \%$ for the $K^{+} K^{-}$, and $23.8 \%$ for the $K^{+} \pi^{0}$ sub-systems.

In the absence of direct $C P V$, the Dalitz plot amplitude $\mathcal{A}$ describing decay of the type $D^{0} \rightarrow(r \rightarrow A B) C$ can be parameterized using an isobar model representation in terms of a sum of amplitudes $A_{r}\left(s_{+}, s_{-}\right)^{1}$ for all relevant SM intermediate states $r$, each with a complex coefficient, i.e., $\mathcal{A}=\sum_{r} a_{r} e^{i \phi_{r}} A_{r}\left(s_{+}, s_{-}\right)$. Here $s_{ \pm}$ are the squared invariant masses of the pair of final state particles with charges $(+$, $0)$ and $(-, 0)$, respectively. The quantities $a_{r}$ and $\phi_{r}$ are, respectively, the magnitude and phase of the complex amplitude-coefficient. The fit fraction for each process $r$ is defined as $f_{r} \equiv \int\left|a_{r} A_{r}\right|^{2} d s_{+} d s_{-} / \int|\mathcal{A}|^{2} d s_{+} d s_{-}$. We model incoherent, $C P$-symmetric background empirically $[25,21]$. In the absence of $C P V$, we expect the values of $a_{r}$, $\phi_{r}$, and $f_{r}$ to be identical for $D^{0}$ and $\bar{D}^{0}$ decay. The results obtained with this assumption are listed in Ref. [25] for $\widetilde{D} \rightarrow \pi^{-} \pi^{+} \pi^{0}$ and in Ref. [21] for $\widetilde{D} \rightarrow K^{-} K^{+} \pi^{0}$. To allow the possibility of $C P V$ in the present analysis, we let a second process - not necessarily of SM origin - contribute to each of the amplitudes $A_{r}$, thus permitting the amplitude-coefficients for $D^{0}$ and $\bar{D}^{0}$ to differ. We summarize the results of the fit to the data in terms of differences in the magnitudes of the amplitudes, phases, and fit fractions $\left(\Delta a_{r}=a_{r}^{\bar{D}^{0}}-a_{r}^{D^{0}}, \Delta \phi_{r}=\phi_{r}^{\bar{D}^{0}}-\phi_{r}^{D^{0}}\right.$, and $\left.\Delta f_{r}=f_{r}^{\bar{D}^{0}}-f_{r}^{D^{0}}\right)$ in Table 7.14 for $\pi^{-} \pi^{+} \pi^{0}$, and in Table 7.15 for $K^{-} K^{+} \pi^{0}$.

\footnotetext{
${ }^{1}$ The amplitude in this form is defined with respect to $D^{0}$. The corresponding $\bar{D}^{0}$ amplitude is $\overline{A_{r}}\left(s_{-}, s_{+}\right)$.
} 


\subsubsection{Results of model fit}

Table 7.14: Model-dependent $C P$ asymmetry in the $\widetilde{D} \rightarrow \pi^{-} \pi^{+} \pi^{0}$ Dalitz plots. The first and second errors are statistical and systematic, respectively. For details on the Dalitz plot parametrization and the $a_{r}, \phi_{r}$, and $f_{r}$ values, see Ref. [25].

\begin{tabular}{lcccc}
\hline \hline State & $f_{r}(\%)$ & $\Delta a_{r}(\%)$ & $\Delta \phi_{r}\left(^{\circ}\right)$ & $\Delta f_{r}(\%)$ \\
\hline$\rho^{+}(770)$ & 68 & $-3.2 \pm 1.7 \pm 0.8$ & $-0.8 \pm 1.0 \pm 1.0$ & $-1.6 \pm 1.1 \pm 0.4$ \\
$\rho^{0}(770)$ & 26 & $2.1 \pm 0.9 \pm 0.5$ & $0.8 \pm 1.0 \pm 0.4$ & $1.6 \pm 1.4 \pm 0.6$ \\
$\rho^{-}(770)$ & 35 & $2.0 \pm 1.1 \pm 0.8$ & $-0.6 \pm 0.9 \pm 0.4$ & $0.7 \pm 1.1 \pm 0.5$ \\
$\rho^{+}(1450)$ & 0.1 & $2 \pm 11 \pm 8$ & $-30 \pm 25 \pm 9$ & $0.0 \pm 0.1 \pm 0.1$ \\
$\rho^{0}(1450)$ & 0.3 & $13 \pm 8 \pm 6$ & $-1 \pm 14 \pm 3$ & $0.1 \pm 0.2 \pm 0.1$ \\
$\rho^{-}(1450)$ & 1.8 & $-3 \pm 6 \pm 5$ & $8 \pm 7 \pm 3$ & $-0.2 \pm 0.3 \pm 0.1$ \\
$\rho^{+}(1700)$ & 4 & $19 \pm 27 \pm 9$ & $9 \pm 7 \pm 3$ & $0.4 \pm 1.0 \pm 0.4$ \\
$\rho^{0}(1700)$ & 5 & $-31 \pm 20 \pm 12$ & $-7 \pm 6 \pm 2$ & $-1.3 \pm 0.8 \pm 0.3$ \\
$\rho^{-}(1700)$ & 3 & $-3 \pm 14 \pm 11$ & $-3 \pm 8 \pm 3$ & $-0.5 \pm 0.6 \pm 0.3$ \\
$f_{0}(980)$ & 0.2 & $0.0 \pm 0.1 \pm 0.2$ & $-3 \pm 7 \pm 4$ & $0.0 \pm 0.1 \pm 0.1$ \\
$f_{0}(1370)$ & 0.4 & $-0.3 \pm 1.3 \pm 1.2$ & $7 \pm 14 \pm 5$ & $-0.2 \pm 0.1 \pm 0.1$ \\
$f_{0}(1500)$ & 0.4 & $0.4 \pm 1.1 \pm 0.7$ & $-1 \pm 12 \pm 1$ & $0.0 \pm 0.1 \pm 0.1$ \\
$f_{0}(1710)$ & 0.3 & $-3 \pm 3 \pm 2$ & $-25 \pm 13 \pm 11$ & $0.0 \pm 0.1 \pm 0.1$ \\
$f_{2}(1270)$ & 1.3 & $8 \pm 4 \pm 5$ & $2 \pm 5 \pm 2$ & $0.1 \pm 0.1 \pm 0.1$ \\
$\sigma(400)$ & 0.8 & $-0.3 \pm 0.7 \pm 2.0$ & $-4 \pm 7 \pm 3$ & $-0.1 \pm 0.1 \pm 0.1$ \\
Nonres & 0.8 & $12 \pm 7 \pm 8$ & $11 \pm 9 \pm 4$ & $0.2 \pm 0.3 \pm 0.2$ \\
\hline \hline
\end{tabular}

Table 7.15: Model-dependent $C P$ asymmetry in the $\widetilde{D} \rightarrow K^{-} K^{+} \pi^{0}$ Dalitz plots. The errors are statistical and systematic, respectively. We show the $a_{0}(980)$ contribution, when it is included in place of the $f_{0}(980)$, in square brackets. For details on the Dalitz plot parametrization and the $a_{r}, \phi_{r}$, and $f_{r}$ values, see Ref. [21]. We use Model-I of Ref. [21] to obtain central values and Model-II for study of systematic errors.

\begin{tabular}{lcccc}
\hline \hline State & $f_{r}(\%)$ & $\Delta a_{r}(\%)$ & $\Delta \phi_{r}\left(^{\circ}\right)$ & $\Delta f_{r}(\%)$ \\
\hline$K^{*}(892)^{+}$ & 45 & $2 \pm 3 \pm 2$ & $10 \pm 12 \pm 3$ & $0.8 \pm 1.1 \pm 0.4$ \\
$K^{*}(1410)^{+}$ & 4 & $101 \pm 65 \pm 37$ & $1 \pm 21 \pm 6$ & $1.7 \pm 1.8 \pm 0.6$ \\
$K^{+} \pi^{0}(S)$ & 16 & $-130 \pm 64 \pm 51$ & $-9 \pm 10 \pm 6$ & $-2.3 \pm 4.7 \pm 1.0$ \\
$\phi(1020)$ & 19 & $-1 \pm 2 \pm 1$ & $-10 \pm 20 \pm 5$ & $-0.4 \pm 0.8 \pm 0.2$ \\
$f_{0}(980)$ & 7 & $14 \pm 16 \pm 6$ & $-12 \pm 25 \pm 8$ & $0.4 \pm 2.6 \pm 0.2$ \\
{$\left[a_{0}(980)^{0}\right]$} & {$[6]$} & {$[19 \pm 16 \pm 6]$} & {$[-7 \pm 16 \pm 8]$} & {$[0.6 \pm 1.9 \pm 0.2]$} \\
$f_{2}^{\prime}(1525)$ & 0.1 & $-38 \pm 74 \pm 8$ & $6 \pm 36 \pm 12$ & $0.0 \pm 0.1 \pm 0.3$ \\
$K^{*}(892)^{-}$ & 16 & $1 \pm 3 \pm 1$ & $-7 \pm 4 \pm 2$ & $1.7 \pm 1.3 \pm 0.4$ \\
$K^{*}(1410)^{-}$ & 5 & $133 \pm 93 \pm 68$ & $-23 \pm 13 \pm 9$ & $1.7 \pm 2.8 \pm 0.7$ \\
$K^{-} \pi^{0}(S)$ & 3 & $8 \pm 68 \pm 36$ & $32 \pm 39 \pm 14$ & $0.4 \pm 2.4 \pm 0.5$ \\
\hline \hline
\end{tabular}



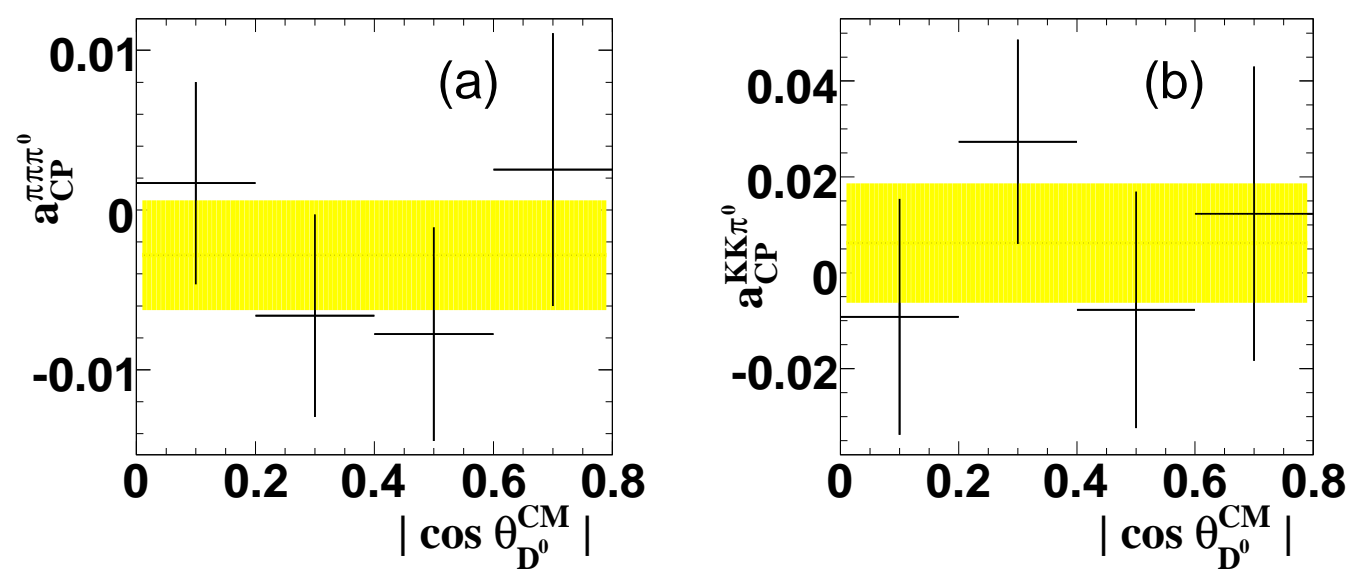

Figure 7.17: Phase-space-integrated $C P$ asymmetry as a function of the cosine of the polar angle of the reconstructed $\widetilde{D}$ candidate CM momentum for (a) $\widetilde{D} \rightarrow \pi^{-} \pi^{+} \pi^{0}$ and (b) $\widetilde{D} \rightarrow K^{-} K^{+} \pi^{0}$ decays. The dashed lines represent the central values, and the shaded regions the $1 \sigma$ intervals.

\subsubsection{Systematic uncertainties}

Systematic uncertainties in the quantities describing $C P$ asymmetries, reported in Tables 7.14-7.15, arise from experimental effects, and also from uncertainties in the the models used to describe the data. We determine these separately, as described in Refs. [25, 21], and add them in quadrature. For all variations described below, we assign the maximum magnitude deviation from the central value as a systematic uncertainty, accounting for correlations among parameters. For resonance lineshapes and form-factors, we vary the parameters [20] by $\pm 1 \sigma$. Similarly, we vary the signal efficiency parameters for $D^{0}$ or $\bar{D}^{0}$ events by $\pm 1 \sigma$, the ratios of particle-identification rates in data and simulation by $\pm 1 \sigma$, and the background shapes using simulation rather than data sidebands. We include uncertainties from $D^{0}-\bar{D}^{0}$ misidentification, estimated from simulation, in experimental systematics.

\subsubsection{Asymmetry in branching ratio vs asymmetry in Dalitz Plot}

To this point we have described the investigation of time-integrated $C P$ asymmetry in neutral $D$ meson decays using information from the Dalitz plot distributions. Any differences in the overall branching fractions for the $D^{0}$ and $\bar{D}^{0}$ decays to $\pi^{-} \pi^{+} \pi^{0}$, $K^{-} K^{+} \pi^{0}$ would also indicate time-integrated $C P V$. This information is not captured by the differential comparisons of Dalitz plot structures already described, and in fact is complementary to them. The overall-rate asymmetry is sensitive only to ratios of $D^{0}$ to $\bar{D}^{0}$ signal yields. To correct for any production asymmetry in $D^{0}$-flavor assignment, we weight each event by the relative efficiency for flavor assignment, as described in Ref. [45]. Since there is an asymmetry [45] between the number of events reconstructed at forward and backward polar angles of the $\widetilde{D}$ candidate CM 
momentum, we extract the $C P$ asymmetry value, $a_{C P} \equiv \frac{N_{\bar{D}^{0}}-N_{D^{0}}}{N_{\bar{D}^{0}}+N_{D^{0}}}$, in intervals of the cosine of this angle. Here $N$ denotes the number of signal events [18]. We show the $a_{C P}$ distribution in Fig. 7.17 and perform $\chi^{2}$ minimization to obtain the central values: $\left(-0.31 \pm 0.41\right.$ (stat.) \pm 0.17 (syst) $\%$ for $\pi^{-} \pi^{+} \pi^{0}$ and $(1.00 \pm 1.67$ (stat.) \pm 0.25 (syst) $\%$ for $K^{-} K^{+} \pi^{0}$ final states. The systematic uncertainties result from signal efficiency, particle-identification, background treatment, and $D^{0}-\bar{D}^{0}$ misidentification. As a consistency check, we repeat the analysis using signal events within $\pm 2.5 \sigma^{2}$ of the reconstructed $D^{0}$ mass peak, and find consistent results: $(-0.28 \pm$ 0.34 (stat) \pm 0.19 (syst)) $\%$ for $\pi^{-} \pi^{+} \pi^{0}$ and $(0.62 \pm 1.24$ (stat) \pm 0.28 (syst)) $\%$ for $K^{-} K^{+} \pi^{0}$

\subsection{Summary}

In summary, our model-independent and model-dependent analyses show no evidence of $C P V$ in either of the SCS $\widetilde{D}$ decay processes studied. With the null results of Ref. [45, 46, 47, 48] for $\widetilde{D} \rightarrow K^{+} K^{-}$and $\widetilde{D} \rightarrow \pi^{+} \pi^{-}$decays, we conclude that any $C P V$ in the charm sector must occur at a rate less than $1 \%$. These results are in accord with the SM predictions, and provide constraints on some models beyond the SM [9].

\footnotetext{
${ }^{2}$ To avoid getting close to the region in one of the sidebands which contains $\widetilde{D} \rightarrow K^{\mp} \pi^{ \pm} \pi^{0}$ events. See Ref. [18] for details.
} 


\section{Bibliography}

[1] I.I. Bigi and A.I. Sandra, Cambridge Monogr. Part. Phys. 9, 1 (2000).

[2] N. Cabibbo, Phys. Rev. Lett. 10, 531 (1963); M. Kobayashi and T. Maskawa, Prog. Theoret. Phys. 49, 652 (1973).

[3] L. Wolfenstein, Phys. Rev. Lett. 51, 1945 (1983).

[4] M. Gronau and D. London, Phys. Lett. B 253, 483 (1991); M. Gronau and D. Wyler, Phys. Lett. B 265, 172 (1991);

[5] D. Atwood, I. Dunietz, and A. Soni, Phys. Rev. Lett. 78, 3257 (1997).

[6] A. Giri, Y. Grossman, A. Soffer and J. Zupan, Phys. Rev. D68, 054018 (2003); A. Bondar, Proceedings of BINP Special Analysis Meeting on Dalitz Analysis, 24-26 Sep. 2002, unpublished.

[7] J.J. Christenson, J.W. Cronin, V.L. Fitch, and R. Turlay, Phys. Rev. Lett. 13, 138 (1964).

[8] A. Pais and S.B. Treiman, Phys. Rev. D12, 2744 (1975).

[9] Y. Grossman, A.L. Kagan, and Y. Nir, Phys. Rev. D75, 036008 (2007).

[10] I.I. Bigi, hep-ph/0104008 (2001).

[11] Alexey A. Petrov, Phys. Rev. D69, 111901 (2004).

[12] B. Aubert et al. (BABAR), Nucl. Instr. and Methods A479, 1 (2002).

[13] H. Albrecht et al. (ARGUS Collaboration), Phys. Lett. B192, 245 (1987); C. Albajar et al. (UA1 Collaboration), Phys. Lett. B186, 247 (1987).

[14] P. Oddone, in Linear Collider B $\bar{B}$ Factory Conceptual Design, proceedings of the Workshop, Los Angeles, California, 1987, edited by D. H. Stork (World Scientific, Singapore, 1987), p. 243.

[15] M.S. Zisman, Ann. Rev. Nucl. Part. Sci. 47, 315 (1997).

[16] P. Burchat et al., Nucl. Instr. and Methods A316, 217 (1992).

[17] J. Schwiening et al. [BABAR Collaboration], Nucl. Instr. and Methods A553, 317 (2005). 
[18] B. Aubert et al. (BABAR), Phys. Rev. D74, 091102 (2006).

[19] R.H. Dalitz, Phil. Mag. 44, 1068 (1953).

[20] W.-M. Yao et al. (PDG), J. Phys. G33, 1 (2006).

[21] B. Aubert et al. (BABAR Collaboration), Phys. Rev. D76, 011102 (R)(2007).

[22] J.L. Rosner, Phys. Rev. D74, 076006 (2006); M.R. Pennington, Int. J. Mod. Phys. A21, 5503 (2006); D. V. Bugg, Phys. Lett. B632, 471 (2006).

[23] E.M. Aitala et al. (E-791 Collaboration), Phys. Rev. Lett. 89, 121801 (2002).

[24] A. Giri, Y. Grossman, A. Soffer, and J. Zupan, Phys. Rev. D68, 054018 (2003).

[25] B. Aubert et al. (BABAR Collaboration), Phys. Rev. Lett. 99, 251801 (2007).

[26] S.J. Lindenbaum and R.M. Sternheimer, Phys. Rev. 105, 1874 (1957); M.G. Olsson and G.V. Yodh, Phys. Rev. 145, 1309 (1966); D.J. Herndon, P. Söding, and R.J. Cashmore, Phys. Rev. D11, 3165 (1975).

[27] J.M. Blatt and W.F. Weisskopf, Theoretical Nuclear Physics, John Wiley \& Sons, New York, 1952.

[28] H. Albrecht et al. (ARGUS Collaboration), Phys. Lett. B308, 435 (1993).

[29] D. Aston et al. (LASS Collaboration), Nucl. Phys. B296, 493 (1988); W.M. Dunwoodie, private communication.

[30] E.M. Aitala et al. (E-791 Collaboration), Phys. Rev. D73, 032004 (2006); B.T. Meadows, private communication.

[31] S.M. Flatte, Phys. Lett. B38, 232 (1972).

[32] M. Ablikim et al. (BES Collaboration), Phys. Lett. B607, 243 (2005).

[33] A. Abele et al. (Crystal Barrel Collaboration), Phys. Rev. D57, 3860 (1998).

[34] B. Aubert et al. (BABAR Collaboration), Phys. Rev. D72, 052008 (2005).

[35] C. Cawlfield et al. (CLEO Collaboration), Phys. Rev. D74, 031108 (2006).

[36] F. Buccella et al., Phys. Rev. D51, 3478 (1995).

[37] C. Zemach, Phys. Rev. 133, B1201 (1964).

[38] A. Poluektov et al. (Belle Collaboration), Phys. Rev. D73, 112009 (2006).

[39] B. Aubert et al. (BABAR Collaboration), Phys. Rev. Lett. 95, 121802 (2005).

[40] Y. Grossman, Z. Ligeti, and A. Soffer, Phys. Rev. D67, 071301(R) (2003).

[41] D. Cronin-Hennessy et al. (CLEO Collaboration), Phys. Rev. D72, 031102 (2005). 
[42] B. Aubert et al. (BABAR Collaboration), Phys. Rev. D72, 071102 (2005).

[43] S. Kopp et al. (CLEO Collaboration), Phys. Rev. D63, 092001 (2001).

[44] Y. Grossman, A. Soffer, and J. Zupan, Phys. Rev. D72, 031501 (2005).

[45] B. Aubert et al. (BABAR Collaboration), arXiv:0709.2715 (to appear in Phys. Rev. Lett.) (2007).

[46] B. Aubert et al. (BABAR Collaboration), arXiv:0712.2249 (submitted to Phys. Rev. D) (2007).

[47] M. Staric et al. (Belle Collaboration), Phys. Rev. Lett. 98, 211803 (2007).

[48] B. Aubert et al. (BABAR Collaboration), Phys. Rev. Lett. 91, 121801 (2003).

[49] D. Asner et al. (CLEO Collaboration), Phys. Rev. D70, 091101 (2004).

[50] K. Mishra (BABAR Collaboration), arXiv:0711.1544, proceedings of the XII International Conference on Hadron Spectroscopy (2007).

[51] D. Asner et al. (CLEO Collaboration), Phys.Rev. D70, 091101 (2004). 